\title{
COHOMOLOGICAL TENSOR FUNCTORS ON REPRESENTATIONS OF THE GENERAL LINEAR SUPERGROUP
}

\author{
TH. HEIDERSDORF, R. WEISSAUER
}

\begin{abstract}
We define and study cohomological tensor functors from the category $T_{n}$ of finite-dimensional representations of the supergroup $G l(n \mid n)$ into $T_{n-r}$ for $0<r \leq n$. In the case $D S: T_{n} \rightarrow T_{n-1}$ we prove a formula $D S(L)=\bigoplus \Pi^{n_{i}} L_{i}$ for the image of an arbitrary irreducible representation. In particular $D S(L)$ is semisimple and multiplicity free. We derive a few applications of this theorem such as the degeneration of certain spectral sequences and a formula for the modified superdimension of an irreducible representation.
\end{abstract}

Part 1. Cohomological tensor functors 7

1. The superlinear groups 7

2. The Duflo-Serganova functor $D S \quad 10$

3. Cohomology Functors 12

4. Support varieties and the kernel of $D S \quad 14$

5. The tensor functor $D \quad 16$

6. The relation between $D S(V)$ and $D(V) \quad 19$

7. Hodge decomposition 24

8. The case $m>1 \quad 26$

9. Boundary maps 30

10. Highest Weight Modules 32

11. The Casimir 36

Part 2. The Main Theorem and its proof 38

12. The language of Brundan and Stroppel 39

13. On segments, sectors and plots 41

14. Mixed tensors and ground states 45

15. Sign normalizations 50

16. The Main theorem 52

17. Strategy of the proof 60

2010 Mathematics Subject Classification: 17B10, 17B20, 17B55, 18D10, $20 \mathrm{G05.}$ 
18. Modules of Loewy length $3 \quad 67$

19. Inductive Control over DS 73

20. Moves 79

Part 3. Consequences of the Main Theorem 89

21. Tannaka Duals $\quad 89$

22. Cohomology I 94

23. Cohomology II 95

24. Cohomology III 98

25. The forest formula 99

26. I-module structure on the cohomology $H_{D S_{n}}^{\bullet} \quad 104$

27. Primitive elements of $H_{D S_{n}}^{\bullet}(V(\mathbf{1})) \quad 110$

28. Kac module of $1 \quad 114$

29. Strict morphisms 116

30. The module $R\left((n)^{n}\right) \quad 119$

31. The basic hook representations $S \quad 122$

References $\quad 127$

\section{INTRODUCTION}

Little is known about the decomposition of tensor products between finitedimensional representations of the general linear supergroup $G l(m \mid n)$ over an algebraically closed field of characteristic 0 . In this article we define and study cohomological tensor functors from the category $T_{n}=\operatorname{Rep}(G l(n \mid n))$ of finite-dimensional representations of $G l(n \mid n)$ to $T_{n-r}$ for $0<r \leq n$. One of our aims is to reduce questions about tensor products between irreducible representations by means of these functors to lower rank cases so that these can hopefully be inductively understood. This is indeed the case for small $n$ as the $G l(1 \mid 1)$-case has been completely worked out in [GQS07] and the $G l(2 \mid 2)$-case is partially controlled by the theory of mixed tensors [Hei14] [HW15a]. Along the way we obtain formulas for the (modified) superdimensions of irreducible representations.

The tensor functors that we study are variants and generalizations of a construction due to Duflo-Serganova [DS05] and Serganova [Ser10]. For any $x \in X=\left\{x \in \mathfrak{g}_{1} \mid[x, x]=0\right\}$, where $\mathfrak{g}_{1}$ denotes the odd part of the underlying Lie superalgebra $\mathfrak{g l}(m \mid n)$, the cohomology of the complex associated to $(V, \rho) \in T_{n}$

$$
\ldots \stackrel{\rho(x)}{\longrightarrow} V \stackrel{\rho(x)}{\longrightarrow} V \stackrel{\rho(x)}{\longrightarrow} V \stackrel{\rho(x)}{\longrightarrow} \ldots
$$


defines a functor $V \mapsto V_{x}: T_{n} \rightarrow T_{n-r}$ (where $r$ is the so-called rank of $x$ ) which preserves tensor products. The category $T_{n}$ splits in two abelian subcategories $T_{n}=\mathcal{R}_{n} \oplus \Pi \mathcal{R}_{n}$ where $\Pi$ denotes the parity shift (lemma 8). We therefore focus on the $\mathcal{R}_{n}$-case and fix a special $x$ of rank 1 in section 2 and denote the corresponding tensor functor $D S: \mathcal{R}_{n} \rightarrow T_{n-1}$. Later in section 3 we refine this construction to define for any $V \in \mathcal{R}_{n}$ a complex

$$
\ldots \stackrel{\partial}{\longrightarrow} \Pi\left(V_{2 \ell-1}\right) \stackrel{\partial}{\longrightarrow} V_{2 \ell} \stackrel{\partial}{\longrightarrow} \Pi\left(V_{2 \ell+1}\right) \stackrel{\partial}{\longrightarrow} \ldots
$$

whose cohomology in degree $\ell$ is denoted by $H^{\ell}(V)$. The representation $D S(V)$ is naturally $\mathbf{Z}$-graded and we have a direct sum decomposition

$$
D S(V)=\bigoplus_{\ell \in \mathbf{Z}} \Pi^{\ell}\left(H^{\ell}(V)\right.
$$

for $G l(n-1 \mid n-1)$-modules $\left.H^{\ell}(V)\right)$ in $\mathcal{R}_{n-1}$. The definition of $D S$ can be easily generalized to the case $x \in X$ of higher rank $r>1$, and we denote the corresponding tensor functors by $D S_{n, n-r}: T_{n} \rightarrow T_{n-r}$. Like the BGG category the category $T_{n}$ has two different duality functors, the ordinary dual ()$^{\vee}$ and the contragredient dual ()*. The tensor functors $D S$ and $D S_{n, n-r}$ are not $*$-invariant in the sense that $D S\left(V^{*}\right) \not z D S(V)^{*}$. We therefore define an analog $D$ of the Dirac operator and we denote the corresponding Dirac cohomology groups by

$$
H_{D}(V)=\operatorname{ker}(D: M \rightarrow M) / \operatorname{Im}(D: M \rightarrow M)
$$

for a certain module $M \in T_{n-1}$ attached to $V$ in section 5. This defines a $*$-invariant tensor functor. It agrees with $D S$ on irreducible modules, but in general gives rise to an analog of Hodge decomposition (proposition 7.1). The definition of Dirac cohomology generalizes easily to define Z-graded tensor functors $\omega_{n, n-r}=\bigoplus_{\ell \in \mathbb{Z}} \omega_{n, n-r}^{\ell}$ whose graded pieces are functors $\omega_{n, n-r}^{\ell}: T_{n} \rightarrow T_{n-r}$ that are described in section 8 .

The second part is devoted to the main theorem 16.1. In the main theorem we give an explicit formula for the image of an irreducible representation $L=L(\lambda)$ in $\mathcal{R}_{n}$ of atypicality $j$ (for $0<j \leq n$ ) under the functor $D S$. Surprisingly, with its natural $\mathbb{Z}$-gradation, the representation

$$
D S(L)=\bigoplus_{i} L_{i}\left[-\delta_{i}\right]
$$

decomposes completely into a finite direct sum of irreducible representations. Here, for certain integers $\delta_{i} \in \mathbb{Z}$, the summands are attached to irreducible $G l(n-1 \mid n-1)$-modules $L_{i} \in \mathcal{R}_{n-1}$, where $L_{i}\left[-\delta_{i}\right]$ denotes the module $\Pi^{\delta_{i}}\left(L_{i}\right) \in T_{n-1}$ concentrated in degree $\delta_{i}$ with respect to the $\mathbb{Z}$-graduation of $D S(L)$. If we ignore the $\mathbb{Z}$-graduation, the module $D S(L) \in T_{n-1}$ always is semisimple and multiplicity free for irreducible $L$. This makes the main 
theorem into an effective tool to reduce questions about tensor products or superdimensions to lower rank cases in the absence of any known branching laws.

To analyse the Z-graded object $D S(L)$ in more detail, we can assume that $L$ is a representation in the principal block containing the trivial representation (the maximally atypical case). In fact, one can inductively reduce the general case to this special case. The irreducible maximal atypical representations $L \in \mathcal{R}_{n}$ can be described in different ways. For the moment it may be sufficient that up to isomorphism they uniquely correspond to spaced forests of rank $n$ in a natural way. By definition, such spaced forests $\mathcal{F}$ are defined by data

$$
\left(d_{0}, \mathcal{T}_{1}, d_{1}, \mathcal{T}_{2}, \cdots, d_{k-1}, \mathcal{T}_{k}\right)
$$

where the $\mathcal{T}_{i}$ for $i=1, \ldots, k$ are rooted planar trees positioned on points of the numberline from left to right. The integer $d_{0}$ specifies the absolute position of the leftmost tree $\mathcal{T}_{1}$ and the natural numbers $d_{i}$ for $i=1, \ldots, k-1$ indicate the distances between the position of the trees $\mathcal{T}_{i}$ and $\mathcal{T}_{i+1}$. Here we allow $d_{i}=0$, i.e. some trees may be positioned at the same point of the numberline. The absolute positions $\delta_{i}=\sum_{j<i} d_{i} \in \mathbb{Z}$ of the planar trees $\mathcal{T}_{i}$ therefore satisfy

$$
\delta_{1} \leq \delta_{2} \leq \cdots \leq \delta_{k}
$$

In particular, $\delta_{1}$ describes the absolute position of the leftmost tree $\mathcal{T}_{1}$ of the forest and $\delta_{k}$ describes the absolute position of the rightmost tree $\mathcal{T}_{k}$ of this forest. Each tree $\mathcal{T}_{i}$ is a planar tree with say $r_{i}$ nodes, among which is the distinguished node defined by the root of the tree. By definition, the rank of the forest $\mathcal{F}$ is the sum $\sum_{i=1}^{k} r_{i}$ of the nodes of all trees. Since in the equivalence above the rank $n$ is fixed, only forests with at most $k \leq n$ trees occur.

This being said, we are now able to describe the summands of the decomposition of $D S(L)$ mentioned above. For simplicity, we still assume $L$ to be maximal atypical. If $L$ corresponds to the spaced forest with trees $\mathcal{T}_{1}, \mathcal{T}_{2}, \ldots, \mathcal{T}_{k}$ in the sense above with the positions at $\delta_{1}, \ldots, \delta_{k}$, then $D S(L)$ has precisely $k$ irreducible constituents $L_{i}\left[-\delta_{i}\right]$ for $i=1, \ldots, k$, so that $L_{i}$ corresponds to the spaced forest $\mathcal{T}_{1}, \ldots, \mathcal{T}_{i-1}, \partial \mathcal{T}_{i}, \ldots, \mathcal{T}_{k}$ of rank $n-1$ where $\partial \mathcal{T}_{i}$ denotes the forest of planar trees obtained from $\mathcal{T}_{i}$ by removing its root. The trees are now at the new positions $\delta_{1}-1, \ldots, \delta_{i-1}-1, \delta_{i}, \ldots, \delta_{i}, \delta_{i+1}+1, \ldots, \delta_{k}+1$ where we use the convention that $\delta_{i}$ denotes the common position of all the trees in $\partial \mathcal{T}_{i}$. In the special case where $\mathcal{T}_{i}$ has only one node, $\partial \mathcal{T}_{i}$ is not defined and will be discarded (together with $\delta_{i}$ ). In other words, in this case the new spaced forest has only $k-1$ trees. 
This description of the Z-graded object $D S(L)$ follows from the results in sections 21 - 24. We introduce spaced forests in section 21 where we describe the dual of an irreducible representation. The $\mathbf{Z}$-grading of $D S(L)$ for maximally atypical $L$ is then obtained in proposition 23.1 and in the general case in proposition 24.2. These results follow from the main theorem and its proof by a careful bookkeeping, but they are considerably stronger and in particular incorporate theorem 16.1 as a special case.

We show $D S_{n, 0}(L) \cong \bigoplus \omega_{n, 0}^{\ell}(L)[-\ell]$ for irreducible maximal atypical representations $L$. From this, as an application of the main theorem, we obtain in theorem 25.1 a nice explicit formula for the Laurent polynomial

$$
\sum_{\ell \in \mathbf{Z}} \operatorname{sdim}\left(\omega_{n, 0}^{\ell}(L)\right) \cdot t^{\ell}
$$

(Hilbert polynomial) attached to the Dirac cohomology tensor functors

$$
\omega_{n, 0}^{\ell}: T_{n} \rightarrow T_{0}
$$

in the case of an irreducible maximal atypical representation $L$.

As already mentioned, the main theorem does not require $L$ to be in the principal block. Applying $D S$ repeatedly $k$-times to an irreducible representation $L=L(\lambda)$ of atypicality $i$ we obtain an isotypical typical representation $m(\lambda) L^{\text {core }}$ in $T_{n-i}$, and $L^{\text {core }}$ only depends on the block of $L$ (section 16). We derive a closed formula for the multiplicity $m(\lambda)$ in section 16 . The multiplicity $m(\lambda)$ can be expressed as

$$
m(\lambda)=\frac{|\mathcal{F}(\lambda)| !}{\mathcal{F}(\lambda) !}
$$

where $\mathcal{F}(\lambda)$ is the spaced forest associated to $L(\lambda),|\mathcal{F}(\lambda)|$ is the number of its nodes and $\mathcal{F}(\lambda)$ ! is the forest factorial 16. This not only implies that the so called modified superdimension of $L$ does not vanish (i.e. the generalized Kac-Wakimoto conjecture), but moreover gives a closed formula for it. The main theorem has a number of other useful applications and we refer the reader to the list given after theorem 16.1.

The proof of the main theorem occupies the entire second part. Build on an involved induction using translation functors, carried out in sections $18-20$, the proof is reduced to the case of ground-states; these are rather specific irreducible modules in a block. For instance, ground states of the principal block are powers of the Berezin determinant. Then, for a block of atypicality $k<n$, we prove in section 14 that every ground state is a Berezin twist of a mixed tensor, an irreducible direct summand in an iterated tensor $X^{\otimes r} \otimes\left(X^{\vee}\right)^{\otimes s}$ where $X$ denotes the standard representation of $G l(n \mid n)$. It is easy to verify directly that the main theorem holds for Berezin powers and 
mixed tensors. In section 18 we study the Loewy structure of translation functors applied to irreducible representations and their behavior under $D S$. We also explain why we can restrict to the maximally atypical case for the proof of the main theorem. In section 19 we prove both parts of the main theorem (semisimplicity and determination of the constituents) under certain assumption on translation functors which are verified in the later section 20 .

In section 26 we discuss the cohomology ring $H_{D S_{n}}^{\bullet}(V(\mathbf{1}))$ for the tensor functor $D S_{n, 0}$. Although the description of the composition factors of an arbitrary Kac module $V(\lambda)$ is much more complicated than that of $V(\mathbf{1})$, we show in lemma 26.8 that there is an isomorphism

$$
H_{D S_{n}}^{\bullet}(V(\mathbf{1})) \cong H_{D S_{n}}^{\bullet+\operatorname{deg}(\rho)}(V(\lambda))
$$

of $I$-modules. In fact the cohomology ring of $V(\mathbf{1})$ can be identified with the Lie algebra homology ring $H_{\bullet}(\mathfrak{g l}(n))$ and defines an exterior algebra $I$ on primitive elements $f_{1}, f_{3}, \ldots, f_{2 n-1}$ so that $I$ acts on the graded cohomology $H_{D S_{n}}^{\bullet}$ of finite dimensional $\mathfrak{g}$-modules. We also discuss the relationship between the cohomology of a Kac module and its irreducible quotient. We show in theorem 26.10 that the induced homomorphism

$$
H_{D S_{n}}^{\nu}(p r): H_{D S_{n}}^{\nu}(V(\lambda)) \rightarrow H_{D S_{n}}^{\nu}(L(\lambda))
$$

is an isomorphism in the top degree and trivial in all lower degrees. In section 27 we describe the elements of $\left.I \cong H_{D S_{n}}^{\bullet} V(\mathbf{1})\right)$ in terms of the representation theory of the superlinear group $G l(n \mid n)$.

Since the image of an irreducible representation under $D S$ is therefore understood, it is natural to look at the image $D S(I)$ of an indecomposable representations $I$. The kernel of $D S$ is the tensor ideal of representations with a filtration by anti-Kac modules by results in section 4 . If $R(\lambda)$ is a mixed tensor we can easily compute $D S(R(\lambda))$. In other cases it is rather complicated to determine $D S$. As an example for the importance of this problem consider the computation of the tensor product between two irreducible representations $L_{1} \otimes L_{2}=\bigoplus_{i} I_{i}$ in indecomposable summands in $\mathcal{R}_{n}$. The decomposition of $D S\left(L_{1}\right)$ and $D S\left(L_{2}\right)$ gives estimates on the number of possible direct summands, but these are rather weak unless something is known about $D S\left(I_{i}\right)$. For an easy example of the use of the cohomological tensor functors in this setting see [HW15a]. In the last sections 28 31 we give a cohomological criterion 31.5 for an indecomposable representation to be equal to the trivial representation. We call an epimorphism $q: V \rightarrow W$ strict, if the induced morphism $\omega(q): \omega(V) \rightarrow \omega(W)$ for the tensor functor $\omega=\omega_{n, 0}: T_{n} \rightarrow s v e c_{k}$ is surjective. We prove in corollary 31.5 that if $Z$ is an indecomposable module with cosocle 1 such that the 
quotient map $q: Z \rightarrow \mathbf{1}$ is strict, then $Z \simeq \mathbf{1}$. Any such representation $Z$ contains extensions of the trivial representations 1 with the other irreducible constituents in the second upper Loewy layer. This leads us to study the cohomology $H^{i}$ of extensions of the trivial representation

$$
0 \longrightarrow S_{\nu} \longrightarrow V \stackrel{q_{V}}{\longrightarrow} \mathbf{1} \longrightarrow 0
$$

for irreducible representations $S_{\nu}$. We show in the key lemma 31.1 that in this case the map $\omega^{0}\left(q_{V}\right)$ vanishes. This is a contradiction to our analysis in section 29 if we suppose that $Z$ is not irreducible.

Most of the results in this article can be rephrased for representations of the supergroup $G l(m \mid n)$ where $m \neq n$. This will be discussed elsewhere.

Acknowledgements. The authors are grateful to the referee for providing useful suggestions.

\section{PART 1. COHOMOLOGICAL TENSOR FUNCTORS}

\section{THE SUPERLINEAR GROUPS}

Let $k$ be an algebraically closed field of characteristic zero. A super vectorspace $V$ over $k$ is a $\mathbb{Z} / 2 \mathbb{Z}$-graded $k$-vectorspace $V=V_{\overline{0}} \oplus V_{\overline{1}}$. Its superdimension is $\operatorname{sim}(V)=\operatorname{dim}\left(V_{\overline{0}}\right)-\operatorname{dim}\left(V_{\overline{1}}\right)$. The parity shift functor $\Pi$ on the category of super vectorspaces over $k$ is defined by $\Pi(V)_{\overline{0}}=V_{\overline{1}}$ and $\Pi(V)_{\overline{1}}=V_{\overline{0}}$ and the parity endomorphism of $V$ is $p_{V}=i d_{V_{\overline{0}}} \oplus-i d_{V_{\overline{1}}}$ in $\operatorname{End}_{k}(V)$.

Conventions on gradings. For $\mathbb{Z}$-graded object $M=\bigoplus_{i} M_{i}$ with objects $M_{i}$ in an additive category $\mathcal{C}$ one has the shifted $\mathbb{Z}$-graded objects $M\langle j\rangle$ defined by $(M\langle j\rangle)_{i}=M_{i+j}$. If $\mathcal{C}$ carries a super structure defined by a functor $\Pi: \mathcal{C} \rightarrow \mathcal{C}$ such that $\Pi \circ \Pi$ is the identity functor, we mainly use the $\mathbb{Z}$-graded objects $M[j]$ defined by $(M[j])_{i}:=\Pi^{j}\left(M_{i+j}\right)$. Considering objects $L$ in $\mathcal{C}$ as graded objects concentrated in one degree, we often consider the $\mathbb{Z}$-graded objects $L[-\ell]$ concentrated in degree $\ell$. In this context, forgetting the $\mathbb{Z}$-grading of $L[\ell]$ for $L \in \mathcal{C}$ and $\ell \in \mathbb{Z}$ gives the object $\Pi^{\ell}(L)$ in $\mathcal{C}$.

The categories $F$ and $T$. Let $\mathfrak{g}=\mathfrak{g l}(m \mid n)=\mathfrak{g}_{\overline{0}} \oplus \mathfrak{g}_{\overline{1}}$ be the general Lie superalgebra. The even part $\mathfrak{g}_{\overline{0}}=\mathfrak{g l}(m) \oplus \mathfrak{g l}(n)$ of $\mathfrak{g l}(m \mid n)$ can be considered as the Lie algebra of the classical subgroup $G_{\overline{0}}=G l(m) \times G l(n)$ in $G=G l(m \mid n)$. By definition a finite-dimensional representation $\rho$ of $\mathfrak{g l}(m \mid n)$ defines a representation $\rho$ of $G l(m \mid n)$, if its restriction to $\mathfrak{g}_{0}$ comes 
from an algebraic representation of $G_{\overline{0}}$, also denoted $\rho$. For the linear supergroup $G=G l(m \mid n)$ over $k$ let $F$ be the category of the super representations $\rho$ of $G l(m \mid n)$ on finite dimensional super vectorspaces over $k$. If $(V, \rho)$ is in $F$, so is $\Pi(V, \rho)$. The morphisms in the category $F$ are the $G$ linear maps $f: V \rightarrow W$ between super representations, where we allow even and odd morphisms with respect to the gradings on $V$ and $W$, i.e morphisms with $f \circ p_{V}= \pm p_{W} \circ f$. For $M, N \in F$ we have $\operatorname{Hom}_{F}(M, N)=$ $\operatorname{Hom}_{F}(M, N)_{\overline{0}} \oplus \operatorname{Hom}_{F}(M, N)_{\overline{1}}$, where $\operatorname{Hom}_{F}(M, N)_{\overline{0}}$ are the even morphisms. Let $T=s \operatorname{Rep}_{\Lambda}(G)$ be the subcategory of $F$ with the same objects as $F$ and $\operatorname{Hom}_{T}(M, N)=\operatorname{Hom}_{F}(M, N)_{\overline{0}}$. Then $T$ is an abelian category, whereas $F$ is not.

The category $\mathcal{R}$. Fix the morphism $\varepsilon: \mathbb{Z} / 2 \mathbb{Z} \rightarrow G_{\overline{0}}=G l(m) \times G l(n)$ which maps -1 to the element $\operatorname{diag}\left(E_{m},-E_{n}\right) \in G l(m) \times G l(n)$ denoted $\epsilon_{m n}$. We write $\epsilon_{n}=\epsilon_{n n}$. Notice that $A d\left(\epsilon_{m n}\right)$ induces the parity morphism on the Lie superalgebra $\mathfrak{g l}(m \mid n)$ of $G$. We define the abelian subcategory $\mathcal{R}=\operatorname{sRep}(G, \varepsilon)$ of $T$ as the full subcategory of all objects $(V, \rho)$ in $T$ with the property $p_{V}=\rho\left(\epsilon_{m n}\right)$; here $\rho$ denotes the underlying homomorphism $\rho: G l(m) \times G l(n) \rightarrow G l(V)$ of algebraic groups over $k$. The subcategory $\mathcal{R}$ is stable under the dualities ${ }^{\vee}$ and ${ }^{*}$. For $G=G l(n \mid n)$ we usually write $T_{n}$ instead of $T$, and $\mathcal{R}_{n}$ instead of $\mathcal{R}$, to indicate the dependency on $n$.

The duality $*$. The Lie superalgebra $\mathfrak{g}=\mathfrak{g l}(m \mid n)$ has a consistent [Kac78] $\mathbb{Z}$-grading $\mathfrak{g}=\mathfrak{g}_{(-1)} \oplus \mathfrak{g}_{(0)} \oplus \mathfrak{g}_{(1)}$, where $\mathfrak{g}_{\overline{0}}=\mathfrak{g}_{(0)}$ and where $\mathfrak{g}_{\overline{1}}=\mathfrak{g}_{(-1)} \oplus \mathfrak{g}_{(1)}$ is defined by the upper triangular block matrices $\mathfrak{g}_{(1)}$ and $\mathfrak{g}_{(-1)}$ by the lower triangular block matrices. The supertranspose $x^{T}$ (see [Sch79], (3.35) and (4.14)]) of a graded endomorphism $x \in \operatorname{End}\left(k^{m \mid n}\right)$ is defined by

$$
x=\left(\begin{array}{cc}
m_{1} & m_{2} \\
m_{3} & m_{4}
\end{array}\right) \mapsto x^{T}=\left(\begin{array}{cc}
m_{1}^{t} & -m_{3}^{t} \\
m_{2}^{t} & m_{4}^{t}
\end{array}\right), x \in \mathfrak{g}
$$

where $m_{i}^{t}$ denotes the ordinary transpose of the matrices $m_{i}$. If we identify $\mathfrak{g}$ and $\operatorname{End}\left(k^{m \mid n}\right)$, then $\tau(x)=-x^{T}$ defines an automorphism of the Lie superalgebra $\mathfrak{g}$ such that $\tau\left(\mathfrak{g}_{(i)}\right)=\mathfrak{g}_{(-i)}$ holds for $i=-1,0,1$. For a representation $M=(V, \rho)$ in $T_{n}$ and homogenous $x$ in $\mathfrak{g}$ the Tannaka dual representation $M^{\vee}=\left(V^{\vee}, \rho^{\vee}\right)$ is the representation $x \mapsto-\rho(x)^{T}$ on $V$, using the supertranpose $\rho(x)^{T}$ of $\rho(x)$ in $\operatorname{End}(V)$. Finally we define the representation $M^{*}=\left(V^{\vee}, \rho^{\vee} \circ \tau\right)$, where $\tau(x)=-x^{T}$ is the automorphism of $\mathfrak{g}$ defined by the supertranspose on $\mathfrak{g}$. See also [BKN09a], 3.4 using a different convention. $V \in \mathcal{R}_{n}$ (see below) implies $V^{*} \in \mathcal{R}_{n}$ by [Bru03], lemma 4.43. For simple and for projective objects $V$ of $T_{n}$ furthermore $V^{*} \cong V$. Also $\left.\left.V^{*}\right|_{G_{\overline{0}}} \cong V\right|_{G_{\overline{0}}}$ for all $V$ in $T_{n}$. Notice that both $\vee$ and $*$ define contravariant functors on $T_{n}$. 
Weights. Consider the standard Borel subalgebra $\mathfrak{b}$ of upper triangular matrices in $\mathfrak{g}$ and its unipotent radical $\mathfrak{u}$. The basis $\Delta$ of positive roots associated to $\mathfrak{b}$ is given by the basis of the positive roots associated to $\mathfrak{b} \cap \mathfrak{g}_{\overline{0}}$ for the Lie algebra $\mathfrak{g}_{0}$ and a single odd root $x$ whose weight will be called $\mu$. If we denote by $e_{i, i}, i=1, \ldots, 2 n$, the linear form which sends a diagonal element $\left(t_{1}, \ldots, t_{2 n}\right)$ to $t_{i}$, then the simple roots in this basis are given by the set $\left\{e_{1,1}-e_{2,2}, \ldots, e_{2 n-1,2 n-1}-e_{2 n, 2 n}\right\}$ with $\mu=e_{n, n}-e_{n+1, n+1}$. The diagonal elements $t=\operatorname{diag}\left(t_{1}, \ldots, t_{n}, t_{n+1}, \ldots, t_{2 n}\right)$ in $G_{\overline{0}}$ act by semisimple matrices on $V$ for any representation $(V, \rho)$ in $T_{n}$. Hence $V$ decomposes into a direct sum of eigenspaces $V=\bigoplus_{\lambda} V_{\lambda}$ for certain characters $t^{\lambda}=$ $t_{1}^{\lambda_{1}} \cdots t_{n}^{\lambda_{n}}\left(t_{n+1}\right)^{\lambda_{n+1}} \cdots\left(t_{2 n}\right)^{\lambda_{2 n}}$. Then write $\lambda=\left(\lambda_{1}, \ldots, \lambda_{n} ; \lambda_{n+1}, \cdots, \lambda_{2 n}\right)$. A primitive weight vector $v$ (of weight $\lambda$ ) in a representation $(V, \rho)$ of $g$ is a nonzero vector in $V$ with the property $\rho(X) v=0$ for $X \in u$ and $\rho(t) v=t^{\lambda}$. An irreducible representation $L$ has a unique primitive weight vector (up to a scalar), the highest weight vector. Its weight $\lambda$ uniquely determines the irreducible module $L$ up to isomorphism in $\mathcal{R}_{n}$. Therefore we write $L=L(\lambda)$.

Kac modules. We put $\mathfrak{p}_{ \pm}=\mathfrak{g}_{(0)} \oplus \mathfrak{g}_{( \pm 1)}$. We consider a simple $\mathfrak{g}_{(0)}$-module as a $\mathfrak{p}_{ \pm}$-module in which $\mathfrak{g}_{(1)}$ respectively $\mathfrak{g}_{(-1)}$ acts trivially. We then define the Kac module $V(\lambda)$ and the AntiKac module $V^{\prime}(\lambda)$ via

$$
V(\lambda)=\operatorname{Ind} d_{\mathfrak{p}_{+}}^{\mathfrak{g}} L_{0}(\lambda), V^{\prime}(\lambda)=\operatorname{Ind}_{\mathfrak{p}_{-}}^{\mathfrak{g}} L_{0}(\lambda)
$$

where $L_{0}(\lambda)$ is the simple $\mathfrak{g}_{(0)}$-module with highest weight $\lambda$. The Kacmodules are universal highest weight modules. $V(\lambda)$ has a unique maximal submodule $I(\lambda)$ and $L(\lambda)=V(\lambda) / I(\lambda)$ [Kac78], prop.2.4.

The Berezin. The Berezin determinant of the supergroup $G=G_{n}$ defines a one dimensional representation $B e r=B e r_{n}$. Its weight is is given by $\lambda_{i}=1$ and $\lambda_{n+i}=-1$ for $i=1, . ., n$. The representation space of $B e r_{n}$ has the superparity $(-1)^{n}$. We denote the trival representation $B e r^{0}$ by 1 .

Ground states. Each $i$-atypical block of $\mathcal{R}_{n}$ contains irreducible representations $L(\lambda)$ of the form

$$
\lambda=\left(\lambda_{1}, \ldots, \lambda_{n-i}, \lambda_{n}, \ldots, \lambda_{n} ;-\lambda_{n}, \ldots,-\lambda_{n}, \lambda_{n+1+i}, \ldots, \lambda_{2 n}\right) .
$$

with $\lambda_{n} \leq \min \left(\lambda_{n-i},-\lambda_{n+1+i}\right)$. We call these the ground states of the block. They will play a major role in our computation of $D S(L)$ in theorem 16.1.

Equivalence. Two irreducible representations $M, N$ on $T$ are said to be equivalent $M \sim N$, if either $M \cong B e r^{r} \otimes N$ or $M^{\vee} \cong \operatorname{Ber}^{r} \otimes N$ holds for some $r \in \mathbb{Z}$. This obviously defines an equivalence relation on the set of isomorphism classes of irreducible representations of $T$. A self-equivalence of $M$ is given by an isomorphism $f: M \cong B e r^{r} \otimes M$ (which implies $r=0$ and $f$ to be a scalar multiple of the identity) respectively an isomorphism 
$f: M^{\vee} \cong \operatorname{Ber}^{r} \otimes M$. If it exists, such an isomorphism uniquely determines $r$ and is unique up to a scalar and we say $M$ is of type (SD). Otherwise we say $M$ is of type (NSD).

Negligible objects. An object $M \in T_{n}$ is called negligible if it is the direct sum of indecomposable objects $M_{i}$ in $T_{n}$ with superdimensions $\operatorname{sdim}\left(M_{i}\right)=$ 0 . The tensor ideal of negligible objects is denotes $\mathcal{N}$ or $\mathcal{N}_{n}$.

\section{The Duflo-Serganova Functor $D S$}

An embedding. Fix some $1 \leq m \leq n$. We view $G_{n-m}=G l(n-m \mid n-m)$ as an 'outer block matrix' in $G_{n}=G l(n \mid n)$ and $G_{1}$ as the 'inner block matrix' as below. Here $G_{0}$ is the empty group. We fix some invertible $m \times m$-matrix $J$ with the property $J=J^{t}=J^{-1}$. For example take $J$ to be the identity matrix $E$, or the matrix with nonzero entries equal to 1 only in the antidiagonal. We furthermore fix the embedding

$$
\varphi_{n, m}: G_{n-m} \times G_{1} \hookrightarrow G_{n}
$$

defined by

$$
\left(\begin{array}{cc}
A & B \\
C & D
\end{array}\right) \times\left(\begin{array}{ll}
a & b \\
c & d
\end{array}\right) \mapsto\left(\begin{array}{cccc}
A & 0 & 0 & B \\
0 & a E & b J & 0 \\
0 & c J & d E & 0 \\
C & 0 & 0 & D
\end{array}\right)
$$

We use this embedding to identify elements in $G_{n-m}$ and $G_{1}$ with elements in $G_{n}$. In this sense $\epsilon_{n}=\epsilon_{n-m} \epsilon_{1}$ holds in $G_{n}$, for the corresponding elements $\epsilon_{n-m}$ and $\epsilon_{1}$ in $G_{n-m}$ resp. $G_{1}$, defined in section 1 .

Two functors. One has a functor $(V, \rho) \mapsto V^{+}=\left\{v \in V \mid \rho\left(\epsilon_{1}\right)(v)=v\right\}$

$$
+: \mathcal{R}_{n} \rightarrow \mathcal{R}_{n-m}
$$

where $V^{+}$is considered as a $G_{n-m}$-module using $\rho\left(\epsilon_{1}\right) \rho(g)=\rho(g) \rho\left(\epsilon_{1}\right)$ for $g \in G_{n-m}$. Indeed $A d\left(\epsilon_{1}\right)(g)=g$ holds for all $g \in G_{n-m}$. The grading on $V$ induces a grading on $V^{+}$by $\left(V^{+}\right)_{\overline{0}}=V_{\overline{0}} \cap V^{+}$and $\left(V^{+}\right)_{\overline{1}}=V_{\overline{1}} \cap V^{+}$. For this grading the decomposition $V^{+}=\left(V^{+}\right)_{\overline{0}} \oplus\left(V^{+}\right)_{\overline{1}}$ is induced by the parity morphism $\rho\left(\epsilon_{n}\right)$ or equivalently $\rho\left(\epsilon_{n-1}\right)$. With this grading on $V^{+}$the restriction of $\rho$ to $G_{n-m}$ preserves $V^{+}$and defines a representation $\left(V^{+}, \rho\right)$ of $G_{n-m}$ in $\mathcal{R}_{n-m}$.

Similarly define $V^{-}=\left\{v \in V \mid \rho\left(\epsilon_{1}\right)(v)=-v\right\}$. With the grading induced from $V=V_{\overline{0}} \oplus V_{\overline{1}}$ this defines a representation $V^{-}$of $G_{n-m}$ in $\Pi \mathcal{R}_{n-m}$. Obviously

$$
\left.(V, \rho)\right|_{G_{n-m}}=V^{+} \oplus V^{-}
$$


The exact hexagon. Fix the following element $x \in \mathfrak{g}_{n}$

$$
x=\left(\begin{array}{cc}
0 & y \\
0 & 0
\end{array}\right) \in \mathfrak{g}_{n} \text { for } y=\left(\begin{array}{cccc}
0 & 0 & \ldots & 0 \\
0 & 0 & \ldots & 0 \\
\ldots & & \ldots & \\
J & 0 & 0 & 0
\end{array}\right)
$$

for the fixed invertible $m \times m$-matrix $J$. Since $x$ is an odd element with $[x, x]=0$, we get

$$
2 \cdot \rho(x)^{2}=[\rho(x), \rho(x)]=\rho([x, x])=0
$$

for any representation $(V, \rho)$ of $G_{n}$ in $\mathcal{R}_{n}$. Notice $d=\rho(x)$ supercommutes with $\rho\left(G_{n-m}\right)$. Furthermore $\rho(x): V^{ \pm} \rightarrow V^{\mp}$ holds as a $k$-linear map, an immediate consequence of $d \rho\left(\varepsilon_{1}\right)=-\rho\left(\varepsilon_{1}\right) d$, i.e. of $\operatorname{Ad}\left(\varepsilon_{1}\right)(x)=-x$. Since $\rho(x) \in \operatorname{Hom}_{F}(V, V)_{1}$ is an odd morphism, $\rho(x)$ induces the following even morphisms (morphisms in $\mathcal{R}_{n-m}$ )

$$
\rho(x): V^{+} \rightarrow \Pi\left(V^{-}\right) \quad \text { and } \quad \rho(x): \Pi\left(V^{-}\right) \rightarrow V^{+} .
$$

The $k$-linear map $\partial=\rho(x): V \rightarrow V$ is a differential and commutes with the action of $G_{n-m}$ on $(V, \rho)$. Therefore $\partial$ defines a complex in $\mathcal{R}_{n-m}$

$$
\stackrel{\partial}{\longrightarrow} V^{+} \stackrel{\partial}{\longrightarrow} \Pi\left(V^{-}\right) \stackrel{\partial}{\longrightarrow} V^{+} \stackrel{\partial}{\longrightarrow} \cdots
$$

Since this complex is periodic, it has essentially only two cohomology groups denoted $H^{+}(V, \rho)$ and $H^{-}(V, \rho)$ in the following. This defines two functors $(V, \rho) \mapsto D_{n, n-m}^{ \pm}(V, \rho)=H^{ \pm}(V, \rho)$

$$
D_{n, n-m}^{ \pm}: \mathcal{R}_{n} \rightarrow \mathcal{R}_{n-m} \text {. }
$$

It is obvious that an exact sequence

$$
0 \longrightarrow A \stackrel{\alpha}{\longrightarrow} B \stackrel{\beta}{\longrightarrow} C \longrightarrow 0
$$

in $\mathcal{R}_{n}$ gives rise to an exact sequences of complexes in $\mathcal{R}_{n-m}$. Hence

Lemma 2.1. The long exact cohomology sequence defines an exact hexagon in $\mathcal{R}_{n-m}$

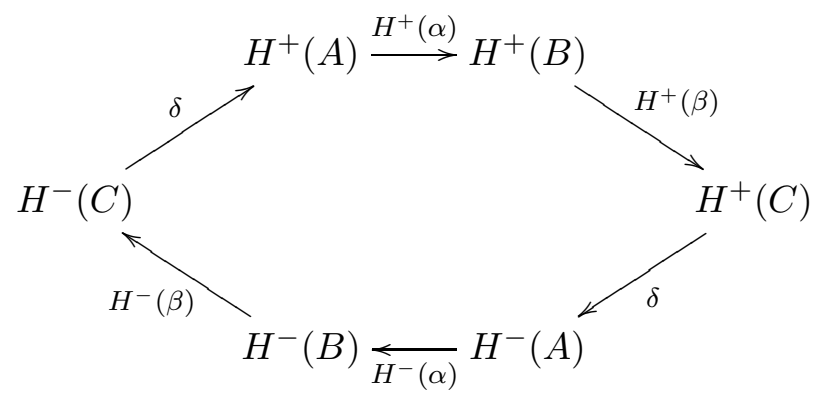


Alternative point of view. For the categories $T=T_{n}$ resp. $T_{n-m}$ (for the groups $G_{n}$ resp. $G_{n-m}$ ) consider the tensor functor of Duflo and Serganova in [DS05]

$$
D S_{n, n-m}: T_{n} \rightarrow T_{n-m}
$$

defined by $D S_{n, n-m}(V, \rho)=V_{x}:=\operatorname{Kern}(\rho(x)) / \operatorname{Im}(\rho(x))$. For $(V, \rho) \in \mathcal{R}_{n}$ we obtain

$$
H^{+}(V, \rho) \oplus \Pi\left(H^{-}(V, \rho)\right)=D S_{n, n-m}(V) .
$$

Indeed, the left side is $D S_{n, n-m}(V)=V_{x}$ for the $k$-linear map $\partial=\rho(x)$ on $V=V^{+} \oplus V^{-}$. Hence $H^{+}$is the functor obtained by composing the tensor functor

$$
D S_{n, n-m}: \mathcal{R}_{n} \rightarrow T_{n-m}
$$

with the functor

$$
T_{n-1} \rightarrow \mathcal{R}_{n-m}
$$

that projects the abelian category $T_{n-m}$ onto $\mathcal{R}_{n-m}$ using

Lemma 2.2. Every object $M \in T_{n}$ decomposes uniquely as $M=M_{0} \oplus$ $M_{1}$ with $M_{0} \in \mathcal{R}_{n}$ and $M_{1} \in \Pi\left(\mathcal{R}_{n}\right)$. This defines a block decomposition of the abelian category

$$
T=\mathcal{R}_{n} \oplus \Pi\left(\mathcal{R}_{n}\right) \text {. }
$$

Proof. For any $M, N \in \mathcal{R}_{n}$ the $\mathbb{Z}_{2}$-graded space $\operatorname{Ext}_{T}^{i}(M, N)$ is concentrated in degree zero [Bru03], Cor. 4.44.

Tensor property. As a graded module over $R=k[x] / x^{2}$ any representation $V$ decomposes into a direct sum of a trivial representation $T$ and copies of $R$ (ignoring shifts by $\Pi$ ). To show that $D S_{n, m}(V)=R_{x} \oplus T_{x}=T$ is a tensor functor, it suffices that $(R \otimes R)_{x}=0$, see also [Ser10]. For this we use that the underlying tensor product is the supertensor product. Indeed for $R=V_{\overline{0}} \oplus V_{\overline{1}}$ and $V_{\overline{0}}=k \cdot 1$ and $V_{\overline{1}}=k \cdot x$ we have $x\left(e_{1}\right)=e_{2}$ and $x\left(e_{2}\right)=0$. The induced superderivation $d$ on $R \otimes R$ satisfies $d(1 \otimes 1)=x \otimes 1+1 \otimes x$, $d(x \otimes 1)=-x \otimes x, d(1 \otimes x)=x \otimes x$ and $d(x \otimes x)=0$. Hence $\operatorname{Im}(d)=$ $\operatorname{Ker}(d)=k \cdot(1 \otimes x+x \otimes 1) \oplus k \cdot x \otimes x$ and therefore $(R \otimes R)_{x}=0$.

\section{COHOMOLOGY FunCTORS}

In this section we assume $V \in T_{n}$ and $m=1$. In the following let $D S$ be the functor $D S_{n, n-1}$ (for $J=1$ ).

Enriched weight structure. The maximal torus of diagonal matrices in $G_{n}$ naturally acts on $D S(V)$ so that $D S(V)$ decomposes into weight spaces $D S(V)=\bigoplus_{\lambda} D S(V)_{\lambda}$ for $\lambda$ in the weight lattice $X(n)$ of $\mathfrak{g}_{n}$. Indeed for the weight decomposition $V=\bigoplus_{\lambda} V_{\lambda}$ every $v \in V$ has the form $v=\sum_{\lambda} v_{\lambda}$ for $v_{\lambda} \in V_{\lambda}$. Now $\partial v=0$ if and only if $\partial v_{\lambda}=0$ holds for all $\lambda$, since 
$\partial\left(V_{\lambda}\right) \subseteq V_{\lambda+\mu}$ for the odd simple weight $\mu$ (ignoring parities on $V$ ). Similarly $v=\partial w$ if and only if $v_{\lambda}=\partial w_{\lambda}$ for all $\lambda$, since we can always project on the weight eigenspaces. This trivial remark shows that $D S(V)$ naturally carries a weight decomposition with respect to the weight lattice $X(n)$ of $\mathfrak{g}_{n}$. The weight structure for $\mathfrak{g}_{n-1}$ is obtained by restriction. The kernel of the restriction $X(n) \rightarrow X(n-1)$ of weights, denoted by

$$
\lambda \mapsto \bar{\lambda},
$$

are the multiples $\mathbb{Z} \cdot \mu$ of the odd simple root $\mu=e_{n, n}-e_{n+1, n+1}$. We may therefore view $D S(V)$ as endowed with the richer weight structure coming from the $G_{n}$-module $V$. This decomposition induces a natural decomposition of $D S(V)$ into eigenspaces $D S(V)=\bigoplus_{\ell} D S(V)_{\ell}$. To make this more convenient consider the torus of elements $\operatorname{diag}\left(1, \ldots, 1,1 ; t^{-1}, 1, \ldots, 1\right)$ for $t \in k^{*}$, called the small torus. These elements commute with $G_{n-1}$ and their eigenvalue decomposition gives a decomposition

$$
V=\bigoplus_{\ell \in \mathbb{Z}} V_{\ell}
$$

into $G_{n-1}$-modules $V_{\ell}$. Here $V_{\ell} \subseteq V$ denotes the subspace defined by all vectors in $V$ on which the above elements of the small torus acts by multiplication with $t^{\ell}$. Obviously $V_{\ell}=0$ for $\ell \notin\left[\ell_{0}, \ell_{1}\right]$ and suitable $\ell_{0}, \ell_{1}$. For the odd morphism $\partial=\rho(x)$ the properties $\mu\left(\operatorname{diag}\left(1, \ldots, 1,1 ; t^{-1}, 1, \ldots, 1\right)\right)=t$ and $\partial\left(V_{\lambda}\right) \subseteq V_{\lambda+\mu}$ show that

$$
\stackrel{\partial}{\longrightarrow} \Pi\left(V_{2 \ell-1}\right) \stackrel{\partial}{\longrightarrow} V_{2 \ell} \stackrel{\partial}{\longrightarrow} \Pi\left(V_{2 \ell+1}\right) \stackrel{\partial}{\longrightarrow} V_{2 \ell+2} \stackrel{\partial}{\longrightarrow}
$$

defines a complex. Its cohomology is denoted $H^{\ell}(V)$. Obviously

$$
\Pi^{\ell}\left(H^{\ell}(V)\right)=D S(V)_{\ell}
$$

and hence we obtain a decomposition of $D S(V, \rho)$ into a direct sum of $G_{n-1^{-}}$ modules

$$
D S(V, \rho)=\bigoplus_{\ell \in \mathbb{Z}} \Pi^{\ell}\left(H^{\ell}(V)\right)
$$

If we want to emphasize the $\mathbb{Z}$-grading, we also write this in the form

$$
D S(V, \rho)=\bigoplus_{\ell \in \mathbb{Z}} H^{\ell}(V)[-\ell] .
$$

We will calculate $D S(L) \in T_{n-1}$ for irreducible $L$ in theorem 16.1 and we will compute its Z-grading in proposition 23.1 and proposition 24.2.

An exact sequence

$$
0 \longrightarrow A \stackrel{\alpha}{\longrightarrow} B \stackrel{\beta}{\longrightarrow} C \longrightarrow 0
$$


in $\mathcal{R}_{n}$ then gives rise to a long exact sequence in $\mathcal{R}_{n-1}$ $\longrightarrow H^{\ell-1}(C) \longrightarrow H^{\ell}(A) \longrightarrow H^{\ell}(B) \longrightarrow H^{\ell}(C) \longrightarrow H^{\ell+1}(A) \longrightarrow$.

Lemma 3.1. For $V$ in $T_{n}$ we have $H^{\ell}\left(B e r_{n} \otimes V\right)=B e r_{n-1} \otimes H^{\ell-1}(V)$. For the Tannaka dual $V^{\vee}$ of $V H^{\ell}(V)^{\vee} \cong H^{-\ell}\left(V^{\vee}\right)$ holds for all $\ell \in \mathbb{Z}$ (isomorphisms of $G_{n-1}$-modules).

Proof. The first property follows from $D S\left(B e r_{n}\right)=B e r_{n-1}[-1]$ and the fact that $D S$ is a tensor functor. Furthermore $D S(V)^{\vee} \cong D S\left(V^{\vee}\right)$, since $D S$ is a tensor functor. Hence the second claim follows from $\left(V^{\vee}\right)_{-\ell}=\left(V_{\ell}\right)^{\vee}$, since $\Pi^{2}$ is the identity and duality 'commutes' with the parity shift $\Pi$.

Note that for $V_{\ell} \in T_{n-1}$ the module $\left(V_{\ell}\right)^{*} \in T_{n-1}$ is isomorphic to $\left(V^{*}\right)_{\ell}$.

Finally, for $(V, \rho) \in \mathcal{R}_{n}$ we get $V^{+}=\bigoplus_{\ell \in 2 \mathbb{Z}} V_{\ell}$ and $\Pi\left(V^{-}\right)=\bigoplus_{\ell \in 1+2 \mathbb{Z}} V_{\ell}$. Hence we obtain the next lemma.

Lemma 3.2. For $V$ in $\mathcal{R}_{n}$ the following holds

$$
H^{+}(V)=\bigoplus_{\ell \in 2 \mathbb{Z}} H^{\ell}(V) \quad, \quad H^{-}(V)=\bigoplus_{\ell \in 1+2 \mathbb{Z}} H^{\ell}(V) .
$$

\section{SUPPORT VARIETIES AND THE KERNEL OF $D S$}

We show that the kernel of $D S$ consists of the modules which have a filtration by AntiKac modules.

Support varieties. We review results from [BKN10], [BKN09b] and [BKN09a] on support varieties. Recall the decomposition $\mathfrak{g}=\mathfrak{g}_{(-1)} \oplus \mathfrak{g}_{(0)} \oplus$ $\mathfrak{g}_{(-1)}$. The support varieties are defined by

$$
V_{\mathfrak{g}_{( \pm 1)}}(M)=\left\{\xi \in \mathfrak{g}_{( \pm 1)} \mid M \text { not projective as a } U(\langle\xi\rangle) \text { - module }\right\} \cup\{0\} \text {. }
$$

Notice that $\xi \in \mathfrak{g}_{( \pm 1)}$ generates an odd abelian Lie superalgebra $\langle\xi\rangle$ with $[\xi, \xi]=0$, which up to isomorphisms has only two indecomposable modules: The trivial module and its projective cover $U(\langle\xi\rangle)$. By [BKN10], prop 6.3 .1

$$
V_{\mathfrak{g}_{( \pm 1)}}(M \otimes N)=V_{\mathfrak{g}_{( \pm 1)}}(M) \cap V_{\mathfrak{g}_{( \pm 1)}}(N) .
$$

The associated variety of Duflo and Serganova is defined as

$$
X_{M}=\left\{\xi \in X \mid M_{\xi} \neq 0\right\}
$$

where $X$ is the cone $X=\left\{\xi \in \mathfrak{g}_{\overline{1}} \mid[\xi, \xi]=0\right\}$. For $\xi \in X$ the condition $M_{\xi} \neq 0$ is equivalent by [BKN09a], 3.6.1, to the condition that $M$ is not projective as a $U(\langle\xi\rangle)$-module. Hence $X_{M}$ is the set of all $\xi \in X$ such that $M$ is not projective as a $U(\langle\xi\rangle)$-module together with $\xi=0$. Thus

$$
V_{\mathfrak{g}_{(-1)}}(M) \cup V_{\mathfrak{g}_{(1)}}(M) \subseteq X_{M} \quad, \quad V_{\mathfrak{g}_{( \pm 1)}}(M)=X_{M} \cap \mathfrak{g}_{( \pm 1)} .
$$


Kac and anti-Kac objects. We denote by $\mathcal{C}^{+}$the tensor ideal of modules with a filtration by Kac modules in $\mathcal{R}_{n}$ and by $\mathcal{C}^{-}$the tensor ideal of modules with a filtration by anti-Kac modules in $\mathcal{R}_{n}$ and quote from [BKN09a], thm 3.3.1, thm 3.3.2

$$
M \in \mathcal{C}^{+} \Leftrightarrow V_{\mathfrak{g}_{(1)}}(M)=0 \quad, \quad M \in \mathcal{C}^{-} \Leftrightarrow V_{\mathfrak{g}_{(-1)}}(M)=0 .
$$

Hence $M$ is projective if and only if $V_{\mathfrak{g}_{(1)}}(M)=V_{\mathfrak{g}_{(-1)}}(M)=0$ holds.

Vanishing criterion. For any $\xi \in X$ there exists $g \in G l(n) \times G l(n)$ and isotropic mutually orthogonal linearly independent roots $\alpha_{1}, \ldots, \alpha_{k}$ such that $A d_{g}(\xi)=\xi_{1}+\ldots+\xi_{m}$ with $\xi_{i} \in g_{\alpha_{i}}$. The number $m=r(\xi)$ is called the rank of $\xi$ [Ser10]. The orbits for the action of $G l(n) \times G l(n)$ on $\mathfrak{g}_{(1)}$ are [BKN09a], 3.8.1

$$
\left(\mathfrak{g}_{(1)}\right)_{m}=\left\{\xi \in \mathfrak{g}_{(1)} \mid r(\xi)=m\right\} \text { for } 0 \leq m \leq n .
$$

By a minimal orbit for the adjoint action of $G l(n) \times G l(n)$ on $g_{( \pm 1)}$ we mean a minimal non-zero orbit with respect to the partial order given by containment in closures. The unique minimal orbit $\left(\mathfrak{g}_{(1)}\right)_{1}$ is the orbit of the element $x$ defined earlier. The situation is analogous for $\mathfrak{g}_{(-1)}$, where $\bar{x}=\tau(x)$ generates the corresponding minimal orbit. A slight modification of [BKN09a], thm 3.7.1 and its proof gives

Theorem 4.1. For $\xi \in \mathfrak{g}_{(1)}$ and $M \in \mathcal{C}^{-}$we have $M_{\xi}=0$. For $\xi \in \mathfrak{g}_{(-1)}$ and $M \in \mathcal{C}^{-}$we have $M_{\xi}=0$. For $\xi=x$ we have $D S(M)=M_{x}=0$ if and only if $M \in \mathcal{C}^{-}$and $M_{\bar{x}}=0$ if and only if $M \in \mathcal{C}^{+}$.

Proof. Let $M \in \mathcal{C}^{-}$. Then the definition of Kac objects implies $V_{\mathfrak{g}_{(1)}}(M)=$ 0 . Hence $\left\{\xi \in \mathfrak{g}_{(1)} \mid M_{\xi} \neq 0\right\}=0$. Conversely assume $M_{x}=0$. Since $V_{\mathfrak{g}_{(1)}}(M)$ is a closed $G l(n) \times G l(n)$-stable variety, it contains a closed orbit. Since the orbits $\left(\mathfrak{g}_{(1)}\right)_{m}$ are closed only for $m=1$, unless $V_{\mathfrak{g}_{(1)}}(M)$ is empty, it must contain $\left(\mathfrak{g}_{(1)}\right)_{1}$. But this would imply $M_{x} \neq 0$, a contradiction. Hence $V_{\mathfrak{g}_{(1)}}(M)=\emptyset$.

Corollary 4.2. For our fixed $x \in\left(\mathfrak{g}_{(1)}\right)_{1}$

(1) $M$ is projective if and only if $M_{x}=0$ and $M_{\tau x}=0$.

(2) $M$ is projective if and only if $M_{x}=0$ and $M_{x}^{*}=0$

(3) If $M=M^{*}$, then $M$ is projective if and only if $M_{x}=0$.

Proof. $M_{x}=0$ implies $V_{\mathfrak{g}_{(1)}}(M)=0$ and $M_{\tau(x)}=0$ implies $V_{\mathfrak{g}_{(-1)}}(M)=0$, hence (1). Now (2) and (3) follow from [BKN09a], 3.4.1 using

$$
V_{\mathfrak{g}_{( \pm 1)}}\left(M^{*}\right)=\tau\left(V_{\mathfrak{g}_{(\mp 1)}}(M)\right) .
$$




\section{THE TENSOR FUNCTOR $D$}

In this section we construct another tensor functor $H_{D}: T_{n} \rightarrow T_{n-1}$ which is defined as the cohomology of a complex given by a Dirac operator $D$. This tensor functor has the advantage that it is compatible with the twisted duality*.

In this section we assume $V \in T_{n}$. For $t \in k^{*}$ the diagonal matrices

$$
\operatorname{diag}\left(E_{n-1}, t, t, E_{n-1}\right) \in G_{\overline{0}}
$$

define a one dimensional torus, the center of $G_{1}$; for this recall the embeddings $G_{1}=i d \times G_{1} \hookrightarrow G_{n-1} \times G_{1} \hookrightarrow G_{n}$. The center of $G_{1}$ commutes with $G_{n-1} \times i d \subset G_{n}$. Hence the center of $G_{1}$ naturally acts on $D S(V)$ in a semisimple way for any representation $(V, \rho) \in T$. Hence the underlying vectorspace $V$ decomposes into $H$-eigenspaces for $H=$ $\operatorname{diag}\left(0_{n-1}, 1,1,0_{n-1}\right)$ in $\mathfrak{g}_{n}=\operatorname{Lie}\left(G_{n}\right)$ which generates the Lie algebra of the torus.

Let $x \in \mathfrak{g}_{n}$ be the fixed nilpotent element specified in section 2. Let $\bar{x}=x^{T}$ denote the supertranspose of $x$. Now $A d\left(\epsilon_{1}\right)(H)=H$ and $[H, x]=$ $[H, \bar{x}]=0$ imply that the operators $\partial=\rho(x)$ and $\bar{\partial}=c \cdot \rho(\bar{x})$ (for any $c \in k^{*}$ ) commute with $H$. Furthermore $[x, \bar{x}]=H$ for the odd elements $x$ and $\bar{x}$ implies

$$
\partial \bar{\partial}+\bar{\partial} \partial=c \cdot \rho(H) .
$$

Since $H$ commutes with $x$, the operator $\rho(H)$ acts on $V_{x}$. Since $H$ commutes with $\varepsilon_{1}$, the grading $V^{ \pm}$is compatible with taking invariants

$$
V^{H}=\{v \in V \mid \rho(H)=0\} .
$$

Similarly we denote the space of coinvariants by $V_{H}$. On $V$ the odd operator $\bar{\partial}$ defines a homotopy of the complex

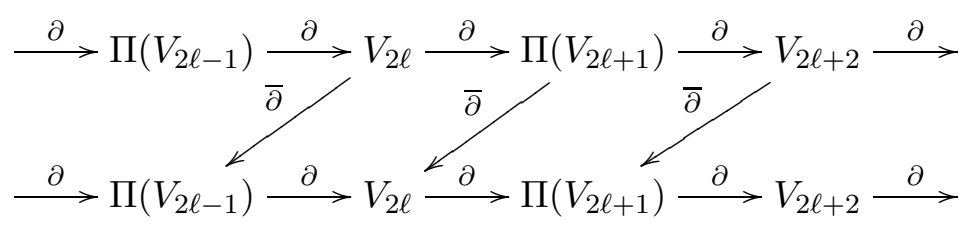

Hence $c \cdot \rho(H)$ is homotopic to zero. In particular, the natural action of $\rho(H)$ on the cohomology modules $H^{\ell}(V)$ is trivial. Therefore

Lemma 5.1. $\rho(H)$ acts trivially on the cohomology $D S(V)=V_{x}$.

Since $H$ acts in a semisimple way, taking $H$-invariants $V \mapsto V^{H}$ is an exact functor and commutes with the cohomology functor $V \mapsto V_{x}$. Thus

$$
D S(V)=M_{x} \quad \text { for } \quad M=V^{H}
$$


and similarly $H^{ \pm}(V)=H^{ \pm}\left(V^{H}\right)$ etc. Notice $\left(V^{H}\right)^{ \pm}=\left(V^{ \pm}\right)^{H}$. Since the operators $\partial$ and $\bar{\partial}$ commute with $H$, they preserve $M=V^{H}$ and anticommute on $M$. In this way we obtain a double complex for $M=V^{H}$ defined by

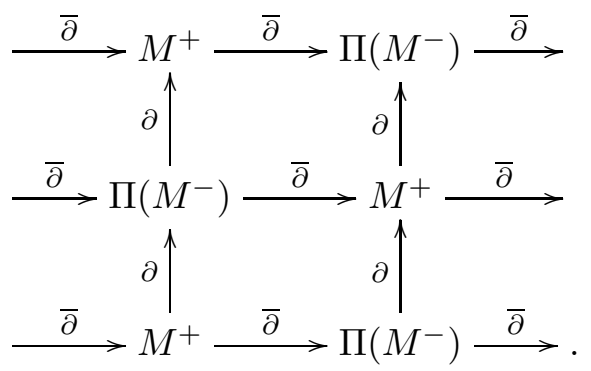

The Dirac operator. This double complex is related to the complex

$$
\cdots \stackrel{D}{\longrightarrow} M^{+} \stackrel{D}{\longrightarrow} \Pi\left(M^{-}\right) \stackrel{D}{\longrightarrow} M^{+} \stackrel{D}{\longrightarrow} \Pi\left(M^{-}\right) \stackrel{D}{\longrightarrow} \cdots
$$

for $M=V^{H}$ attached to the Dirac operator

$$
D=\partial+\bar{\partial} .
$$

Since $M=M^{+} \oplus \Pi\left(M^{-}\right)$, the two cohomology modules $H_{D}^{+}(V)$ and $H_{D}^{-}(V)$ of this periodic complex compute

$$
H_{D}(V)=\operatorname{Kern}(D: M \rightarrow M) / \operatorname{Im}(D: M \rightarrow M)
$$

in the sense that

$$
H_{D}(V)=H_{D}^{+}(V) \oplus \Pi\left(H_{D}^{-}(V)\right)
$$

gives the decomposition of $H_{D}(V)$ into its $\mathcal{R}_{n}$ and $\Pi\left(\mathcal{R}_{n}\right)$-part.

Remark. Note that $D$ commutes with $\rho(H)$. Hence the operator $D$ respects the eigenspaces of $H$ on $V$. Since $D^{2}=\partial^{2}+(\partial \bar{\partial}+\partial \bar{\partial})+\bar{\partial}^{2}=$ $(\partial \bar{\partial}+\partial \bar{\partial})=c \cdot \rho(H)$, we have $\operatorname{Ker}(D: V \rightarrow V)=\operatorname{Kern}\left(D: V^{H} \rightarrow V^{H}\right)$. However $D(V)$ is in general different from $D\left(V^{H}\right)$, although both spaces have the same intersection with $V^{H}$.

Lemma 5.2. For $c=i$ there exist natural isomorphisms $H_{D}\left(V^{*}, \rho^{*}\right) \cong$ $H_{D}(V, \rho)^{*}, H_{D}(V)^{\vee} \cong H_{D}\left(V^{\vee}\right), H_{D}^{ \pm}\left(V^{*}\right) \cong H_{D}^{ \pm}(V)^{*}$ and $H_{D}^{ \pm}\left(V^{\vee}\right) \cong H_{D}^{ \pm}(V)^{\vee}$ of $G_{n-1}$-modules. For short exact sequences in $\mathcal{R}_{n}$ one obtains an exact hexagon in $\mathcal{R}_{n-1}$ for the functors $H_{D}^{ \pm}$.

Proof. The assertion $H_{D}(V)^{\vee}=H_{D}\left(V^{\vee}\right)$ follows since $H_{D}$ is a tensor functor by lemma 5.4. We calculate $\tau(x+i \bar{x})=-(\bar{x}-i x)=i(x+i \bar{x})$, since $\tau^{2}(x)=-x$. Now recall that $\rho^{*}(D)=\rho^{\vee}(\tau(D))=i \rho^{\vee}(D)$ is defined as endomorphism on $V^{*}=V^{\vee}$. Hence $H_{D}\left(V^{*}, \rho^{*}\right)$, by definition the cohomology of $\rho^{*}(D)$ on $\left(V^{*}\right)^{H}$, can be identified with the space

$$
\operatorname{Ker}\left(i \rho^{\vee}(D):\left(V^{\vee}\right)^{H} \rightarrow\left(V^{\vee}\right)^{H}\right) / \operatorname{Im}\left(i \rho^{\vee}(D):\left(V^{\vee}\right)^{H} \rightarrow\left(V^{\vee}\right)^{H}\right) .
$$


Of course we can ignore the factor $i$, and identify this representation with the representation on

$$
\left(\operatorname{Ker}\left(\rho(D): V_{H} \rightarrow V_{H}\right) / \operatorname{Im}\left(\rho(D):\left(V_{H} \rightarrow V_{H}\right)\right)^{\vee}\right.
$$

or hence with

$$
H_{D}(V, \rho)^{\vee}=\left(\operatorname{Ker}\left(\rho(D): V^{H} \rightarrow V^{H}\right) / \operatorname{Im}\left(\rho(D):\left(V^{H} \rightarrow V^{H}\right)\right)^{\vee},\right.
$$

using the dual $\left(V_{H}\right)^{\vee} \rightarrow\left(V^{H}\right)^{\vee}$ of the natural morphism $V^{H} \rightarrow V_{H}$, which is an isomorphism by the semisimplicity of $H$. Finally recall $H_{D}(V, \rho)^{\vee}=$ $H_{D}(V, \rho)^{*}$ for the underlying representation spaces. This is an isomorphism of $G_{n-1}$-modules since $\tau$ restricts to the corresponding $\tau$ on $G_{n-1}$.

So from now on assume $c=i$. Then, in contrast to lemma 3.1, we obtain

Lemma 5.3. There exist natural isomorphisms of functors $\mathcal{R}_{n} \rightarrow \mathcal{R}_{n-1}$

$$
\mu_{V}: H_{D}^{ \pm}\left(V^{*}\right) \cong H_{D}^{ \pm}(V)^{*} \text {. }
$$

Proof. It remains to show that the isomorphism $\mu_{V}: H_{D}^{ \pm}\left(V^{*}\right) \cong H_{D}^{ \pm}(V)^{*}$ given above defines a natural transformation. For a $G_{n}$-linear map $f: V \rightarrow$ $W$ the induced map $f^{*}: W^{*} \rightarrow V^{*}$ is nothing but the morphism $f^{\vee}: W^{\vee} \rightarrow$ $V^{\vee}$, using $V^{*}=V^{\vee}$ and $W^{*}=W^{\vee}$. This now easily shows that the above identifications $\mu_{V}, \mu_{W}$ induce a commutative diagram

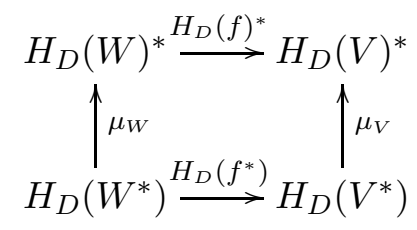

Example. Let $V$ be the Kac module $V(\mathbf{1})$ in $\mathcal{R}_{1}$. Then $D S\left(V^{*}\right)=0$ and $D S(V)=\mathbf{1} \oplus \Pi(\mathbf{1})$. On the other hand $H_{D}(V)=0$ and $H_{D}\left(V^{*}\right)=0$.

Remark. It is not a priori clear how to define a Dirac analog of the modules $H^{\ell}(V)$. Indeed $\bar{\partial}$ and $\partial$ (in the sense of odd morphisms) satisfy $\bar{\partial}: V_{\lambda} \rightarrow V_{\lambda-\mu}$ and $\partial: V_{\lambda} \rightarrow V_{\lambda+\mu}$ for the odd simple weight $\mu$. Hence $\bar{\partial}: V_{\ell} \rightarrow V_{\ell-1}$ and $\partial: V_{\ell} \rightarrow V_{\ell+1}$ and therefore $D=\partial+\bar{\partial}$ does not simply shift the grading. We adress this question in section 7 .

$H_{D}$ as a tensor functor. Although taking $H$-invariants $V \mapsto M=V^{H}$ is not a tensor functor, $H_{D}$ is nevertheless a tensor functor. To show this it is enough to restrict the representations $(V, \rho)$ to $G_{1} \hookrightarrow G_{n}$. Hence it suffices to show that the functor

$$
H_{D}: T_{1} \rightarrow T_{0}=\operatorname{svec}_{k}
$$


is a tensor functor. $H$ generates the center of $\mathfrak{g l}(1 \mid 1)$ and $D^{2}=\rho(H)$. Hence $\operatorname{Kern}(D) \subset V^{H}$. Since $H$ is semisimple, the Jordan blocks of $D$ on $V$ (ignoring the grading!) are Jordan blocks $B_{\lambda}$ of length 1 except for the eigenvalue $\lambda=0$, where they are either Jordan blocks $B_{0}$ of length 1 or Jordan blocks $R$ of lenght 2 . Indeed the square of an indecomposable Jordan block of length $a$ and eigenvalue $\lambda$ is again an indecomposable Jordan block of length $a$ for $\lambda \neq 0$. Since $D^{2}=\rho(H)$ is semisimple, this implies $\lambda=0$ and $a \leq 2$ for $a>1$. By definition, for $V=\bigoplus_{\lambda} k_{\lambda}(V) \cdot B_{\lambda} \oplus k(V) \cdot R$ we have $H_{D}(V)=k_{0}(V) \cdot B_{0}$, if we ignore the grading. Now $B_{\lambda} \otimes B_{\lambda^{\prime}}=B_{\lambda \pm \lambda^{\prime}}$, where the sign depends on the parity of $B_{\lambda}$. Furthermore the characteristic polynomial of $D$ on $R \otimes B_{\lambda}$ is $X^{2}-\lambda^{2}$, hence $D$ has eigenvalue 0 on $R \otimes B_{\lambda}$ only for $\lambda=0$, in which case $R \otimes B_{\lambda}$ is isomorphic to $R$. Finally $R \otimes R \cong R^{2}$. Hence the only possible deviation from the tensor functor property for $H_{D}$ might come from tensor products $B_{\lambda} \otimes B_{\lambda^{\prime}}$ where $\lambda \pm \lambda^{\prime}=0$. In this case $H=\lambda^{2} \cdot i d$ on $B_{\lambda}$ and $B_{\lambda^{\prime}}$, hence $H=2 \lambda^{2} \cdot i d$ on $B_{\lambda} \otimes B_{\lambda^{\prime}}$. But the even operator $D^{2}$ then acts by $2 \lambda^{2} \cdot i d$ on $B_{\lambda} \otimes B_{\lambda^{\prime}}$. Hence $D$ does not have the eigenvalue zero on $B_{\lambda} \otimes B_{\lambda^{\prime}}$ unless $\lambda=\lambda^{\prime}=0$. Therefore $B_{0} \otimes B_{0} \cong B_{0}$ is the only relevant case. Hence $H_{D}(V \otimes W)=k_{0}(V) k_{0}(W) \cdot B_{0}=k_{0}(V)$. $B_{0} \otimes k_{0}(W) \cdot B_{0}=H_{D}(V) \otimes H_{D}(W)$. This remains true if we also take into account gradings.

Lemma 5.4. $H_{D}: T_{n} \rightarrow T_{n-1}$ is a tensor functor.

\section{The RELATION BETWEen $D S(V)$ AND $D(V)$}

For $(V, \rho) \in T_{n}$ the eigenvalue decomposition with respect to the small torus gives a decomposition

$$
V=\bigoplus_{\ell \in \mathbb{Z}} V_{\ell}
$$

into $G_{n-1}$-modules $V_{\ell}$. Furthermore $\bar{\partial}$ and $\partial$ (in the sense of odd morphisms) satisfy $\bar{\partial}: V_{\ell} \rightarrow V_{\ell-1}$ and $\partial: V_{\ell} \rightarrow V_{\ell+1}$. In other words, they give rise to morphisms $\bar{\partial}: \Pi^{\ell}\left(V_{\ell}\right) \rightarrow \Pi^{\ell-1}\left(V_{\ell-1}\right)$ and $\partial: \Pi^{\ell}\left(V_{\ell}\right) \rightarrow \Pi^{\ell+1}\left(V_{\ell+1}\right)$, hence induce morphisms on $\bigoplus_{\ell \in \mathbb{Z}} H^{\ell}(V)$ which shift the grading by -1 resp. +1 .

Since the generator $H$ of the center of $\operatorname{Lie}\left(G_{1}\right)$ commutes with the small torus, we obtain an induced decomposition for the invariant subspace $M=$ $V^{H} \subseteq V$

$$
M=\bigoplus_{\ell} \Pi^{\ell}\left(M_{\ell}\right)
$$

for $\Pi^{\ell}\left(M_{\ell}\right)=M \cap V_{\ell}=\left(V_{\ell}\right)^{H}$. Notice $M=M^{+} \oplus \Pi\left(M^{-}\right)$for $(V, \rho) \in \mathcal{R}_{n}$, with $M^{+}$and $M^{-}$defined in $\mathcal{R}_{n}$ by

$$
M^{+}=\bigoplus_{\ell \in 2 \mathbb{Z}} M_{\ell} \quad, \quad M^{-}=\bigoplus_{\ell \in 1+2 \mathbb{Z}} M_{\ell}
$$


The spaces $M_{\ell}$ are $G_{n-1}$-modules.

On $M$ the operators $\partial$ and $\bar{\partial}$ define even morphisms and they anticommute in the diagram below. Hence we get a double complex $K=K^{\bullet \bullet \bullet}$ in $T_{n-1}$ attached to $(V, \rho)$

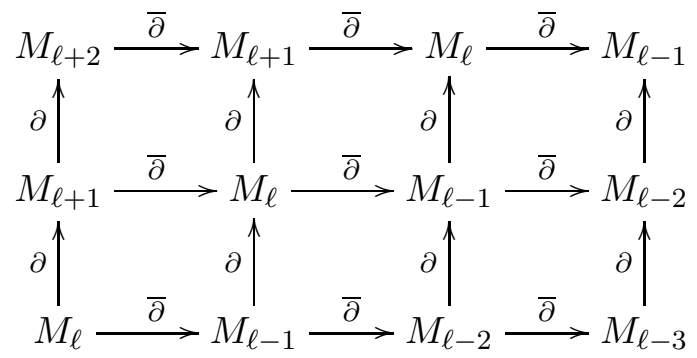

with $K^{i, j}=M_{j-i}$. This double complex is periodic with respect to $(i, j) \mapsto$ $(i+1, j+1)$. The modules $K^{i, j}$ vanish for $j-i \notin\left[\ell_{0}, \ell_{1}\right]$ and certain $\ell_{0}, \ell_{1} \in \mathbb{Z}$.

The associated single complex $(\operatorname{Tot}(K), D)$ has the objects $\operatorname{Tot}(K)^{n}=$ $\bigoplus_{i \in \mathbb{Z}} M_{n+2 i}$ and the differential $D=\partial+\bar{\partial}$. The total complex therefore is periodic with $\operatorname{Tot}^{0}(K)=M^{+}$and $\operatorname{Tot}^{1}(K)=\Pi\left(M^{-}\right)$and computes the cohomology $H^{n}(\operatorname{Tot}(K), D)=H_{D}^{+}(V)$ for $n \in 2 \mathbb{Z}$ and $H^{n}(\operatorname{Tot}(K), D)=$ $H_{D}^{-}(V)$ for $n \in 1+2 \mathbb{Z}$.

On the total complex $(\operatorname{Tot}(K), D)$ we have a decreasing filtration defined by $F^{p} \operatorname{Tot}^{n}(K)=\bigoplus_{r+s=n, r \geq p} K^{r, s}$. This filtration induces decreasing filtrations on the cohomology of the total complex

$$
\cdots \supseteq F^{p}\left(H_{D}^{ \pm}(V)\right) \supseteq F^{p+1}\left(H_{D}^{ \pm}(V)\right) \supseteq \cdots
$$

and a spectral sequence $\left(E_{r}^{p, q}, d_{r}\right)$ converging to

$$
E_{\infty}^{p, q}=g r^{p} H^{p+q}(\operatorname{Tot}(K), D) .
$$

Indeed the convergence of the sequence follows from the fact that the higher differentials $d_{r}: E_{r}^{p q} \rightarrow E_{r}^{p+r, q-r+1}$ vanish for $2 r-(q-p+1)>\ell_{1}-\ell_{0}$. The $E_{1}$-complex of the spectral sequence is the direct sum over all $q$ of the horizontal complexes $E_{1}^{p, q}=\left(H_{\partial}^{q}\left(K^{p, \bullet}\right), \bar{\partial}\right)$. For the various $q$ these complexes are the same up to a shift of the complex. So, if we ignore this shift, these complexes are given by the natural action of $\bar{\partial}$ on $\bigoplus_{\ell \in \mathbb{Z}} H^{\ell}(V)$ defining the complex

$$
\ldots \stackrel{\bar{\partial}}{\longrightarrow} H^{q+1}(V) \stackrel{\bar{\partial}}{\longrightarrow} H^{q}(V) \stackrel{\bar{\partial}}{\longrightarrow} H^{q-1}(V) \stackrel{\bar{\partial}}{\longrightarrow} \ldots .
$$

The decreasing filtration $F^{p}$ induced on

$$
E_{1}(K)^{n}=\bigoplus_{i \in \mathbb{Z}} H^{n+2 i}(V)
$$


has graded terms $\operatorname{gr}^{p}\left(E_{1}(K)^{n}\right)=H_{\partial}\left(K^{p, n-p}\right)=H_{\partial}\left(M_{n-2 p}\right)=H^{n-2 p}(V)$. We now define the subquotient $H_{D}^{n-2 p}(V):=g^{p}\left(E_{\infty}(K)^{n}\right)$ of $H^{n-2 p}(V)$, hence

$$
H_{D}^{\ell}(V):=g r^{p}\left(E_{\infty}(K)^{\ell+2 p}\right) .
$$

Note that this definition does not depend on the choice of $p$. We thus obtain

Lemma 6.1. For $T \in T_{n}$ the cohomology modules $H_{D}^{ \pm}(V)$ admit canonical decreasing filtrations $F^{p}$ whose graded pieces are the $G_{n-1}$-modules $H_{D}^{-2 p}(V)$ for $H_{D}^{+}(V)$ and $H_{D}^{-2 p-1}(V)$ for $H_{D}^{-}(V)$.

Condition $\mathrm{T}$. We say that condition $\mathrm{T}$ holds for $(V, \rho)$ in $T_{n}$ if the natural operation of the operator $\bar{\partial}=\rho(\tau(x))$ on $D S(V, \rho)$ is trivial.

Example. The standard representation $X=X_{s t}$ of $G_{n}$ on $k^{n \mid n}$ satisfies condition $\mathrm{T}$.

Remark. If $\tau(x)$ act trivially both on $D S(V)$ and $D S(W)$ for some $V, W \in T_{n}$, then $\tau(x)$ acts trivially on $D S(V \otimes W)=D S(V) \otimes D S(W)$. If $\tau(x)$ acts trivially on $D S(U)$ for $U \in T_{n}$, then $\tau(x)$ act trivially on every retract of $D S(U)$. Hence condition $\mathrm{T}$ for $(V, \rho)=L(\lambda)$ implies condition $\mathrm{T}$ for every retract $U$ of $D S(V \otimes W)$. Thus the subcategory of objects in $\mathcal{R}_{n}$ satisfying condition $\mathrm{T}$ is closed under tensor products and retracts.

Now consider the following conditions for $(V, \rho)$ :

(1) $(V, \rho)$ is irreducible.

(2) $H^{+}(V) \oplus H^{-}(V)$ is multiplicity free.

(3) $H^{+}(V)$ and $H^{-}(V)$ do not have common constituents.

(4) Condition T holds.

(5) $\bar{\partial}$ acts trivially on $D S(V)$.

(6) The $E_{1}^{p, q}$ and the $E_{2}^{p, q}$ terms of the spectral sequence coincide

$$
H_{\bar{\partial}}\left(H^{\ell}(V)\right)=H^{\ell}(V)
$$

where $\ell:=n-2 p=q-p$.

Later in theorem 16.1 we prove that (1) implies (2). Furthermore it is trivial that $(2) \Longrightarrow(3) \Longrightarrow(4) \Longrightarrow(5) \Longrightarrow(6)$.

Proposition 6.2. If condition (3) holds, then the spectral sequence degenerates at the $E_{1}$-level and $H_{D}^{ \pm}(V)$ is naturally isomorphic to $H^{ \pm}(V)$.

Proof. The differentials of the spectral sequence $d_{r}: E_{r}^{p q} \rightarrow E_{r}^{p+r, q-r+1}$ define maps from the subquotient $E_{r}^{p q}$ of $H^{n-2 p}(V)$ (for $n=p+q$ ) to the subquotient $E_{r}^{p+r, q-r+1}$ of $H^{n-2 p-2 r+1}(V)$. If $H^{n-2 p}(V)$ contributes to $H^{ \pm}(V)$, then $H^{n-2 p-2 r+1}(V)$ contributes to $H^{\mp}(V)$. Since all the higher differentials are $G_{n-1}$-linear, condition (3) forces all differentials $d_{r}$ to be zero for $r \geq 1$. Hence the spectral sequence degenerates at the $E_{1}$-level. 
Proposition 6.3. The spectral sequence always degenerates at the $E_{2}$ level, i.e. for all objects $(V, \rho)$ in $T_{n}$ we have

$$
H_{\bar{\partial}}\left(H^{\ell}(V)\right) \cong H_{D}^{\ell}(V) \text {. }
$$

Corollary 6.4. The kernel of $H_{D}: T_{n} \rightarrow T_{n-1}$ contains $\mathcal{C}^{+} \cup \mathcal{C}^{-}$.

Remark. It seems plausible that the kernel equals $\mathcal{C}^{+} \cup \mathcal{C}^{-}$.

Proof. This is a general assertion on spectral sequences arising from a double complex $K$ such that $K^{i, j}=M_{j-i}$ for maps $\partial: M_{\ell} \rightarrow M_{\ell+1}$ and $\bar{\partial}: M_{\ell} \rightarrow M_{\ell-1}$ between finite dimensional $k$-vectorspaces $M_{\ell}, \ell \in \mathbb{Z}$ so that $M_{\ell}=0$ for almost all $\ell$. Indeed, any such double complex $K$ can be viewed as an object in the category $T_{1}$ via the embedding $\varphi_{n, m}$ of section 2 .. Using $T_{1}=\mathcal{R}_{1} \oplus \Pi\left(\mathcal{R}_{1}\right)$ we can decompose and assume without restriction of generality that it is an object in $\mathcal{R}_{1}$. However, then it defines a maximal atypical object in the category $\mathcal{R}_{1}^{1} \subset \mathcal{R}_{1}$. For this notice that $\mathcal{R}_{1}^{1}$ can be identified with the category of objects in $\mathcal{R}_{1}$ with trivial central character. Note that this condition on the central character for a representation $(V, \rho)$ of $G_{1}$ simply means $V=V^{H}=M$, since $H$ generates the center of $\operatorname{Lie}\left(G_{1}\right)$. This reduces our claim to the special case $n=1$ for $(V, \rho)$ in $\mathcal{R}_{1}^{1}$. Obviously we can assume that $(V, \rho)$ is indecomposable.

The indecomposable objects $V$ in $\mathcal{R}_{1}^{1}$ were classified by Germoni [Ger98]. Either $V \in \mathcal{C}^{+}$(Kac object), or $V \in \mathcal{C}^{-}$or there exists an object $U \subset V, U \in$ $\mathcal{C}^{-}$with irreducible quotient $L$ or there exists a quotient $Q$ of $V$ in $\mathcal{C}^{-}$with irreducible kernel $L^{\prime}$. Since $D S(N)=0$ for all objects in $\mathcal{C}^{-}$(theorem 4.1), we conclude from the long exact sequence of $H^{\ell}$-cohomology that we can either assume $V \in \mathcal{C}^{+}$or that $V$ is irreducible, since in the remaining cases $D S(V)=0$ or $D S(V) \cong D S(L)$ or $D S\left(L^{\prime}\right) \cong D S(V)$. As already mentioned, by the later theorem 16.1 for irreducible $V$, the spectral sequence already abuts. For $r=1$ however this is obvious anyway, since any atypical irreducible $L$ is isomorphic to a Berezin power $L \cong B e r^{m}$. Hence $H_{D}^{\nu}(L)=H^{\nu}(L)=k$ for $\nu=m$ and $H_{D}^{\nu}(L)=H^{\nu}(L)=0$ otherwise.

So it remains to consider the case of indecomposable Kac objects $V \in \mathcal{C}^{+}$ in $\mathcal{R}_{1}^{1}$. Unless $V \in \mathcal{C}^{+} \cap \mathcal{C}^{-}$, by Germoni's results $V \cong V(i ; m)$ for $i \in \mathbb{Z}$ and $m \in \mathbb{N}$ is a successive extension

$$
0 \rightarrow V(i-2 ; m-1) \rightarrow V(i ; m) \rightarrow V\left(\text { Ber }^{i}\right) \rightarrow 0
$$

of the Kac objects with $V(i ; 1)=V\left(B e r^{i}\right)$. Furthermore the Kac module $V\left(B e r^{i}\right)$ is an extension of Berezin modules

$$
0 \rightarrow \mathrm{Ber}^{i-1} \rightarrow V\left(\mathrm{Ber}^{i}\right) \rightarrow \mathrm{Ber}^{i} \rightarrow 0
$$


hence $H^{\ell}\left(V\left(B e r^{i}\right)\right) \cong k$ for $\ell=i, i-1$ and is zero otherwise. From the long exact cohomology sequence and induction we obtain $\operatorname{dim}\left(H^{\nu}(V(i ; m))=1\right.$ for $\nu \in\{i, i-1, \ldots, i-2 m+1\}$, and $H^{\nu}(V(i ; m))=0$ otherwise. So (for fixed $q)$ the complexes in the $E_{1}$-term of the spectral sequence for $V=V(i ; m)$ have the form

$$
0 \rightarrow H^{i}(V) \rightarrow H^{i-1}(V) \rightarrow \cdots \rightarrow H^{i-2 m+2}(V) \rightarrow H^{i-2 m+1}(V) \rightarrow 0
$$

with differentials $\bar{\partial}$ and $H^{\nu}(V)$ of dimension one for $\nu=i, i-1, \ldots, i-2 m+1$. We have to show that these complexes are acyclic for all $V=V(i ; m)$. For this it suffices that the first differential $\bar{\partial}: H^{i}(V) \rightarrow H^{i-1}(V)$, the third differential $\bar{\partial}: H^{i-2}(V) \rightarrow H^{i-3}(V)$ and so on, are injective. By dimension reasons the differentials $\bar{\partial}: H^{i-1}(V) \rightarrow H^{i-2}(V), \bar{\partial}: H^{i-3}(V) \rightarrow H^{i-4}(V)$ etc. are then isomorphisms and the differentials $\bar{\partial}: H^{i}(V) \rightarrow H^{i-1}(V)$, $\bar{\partial}: H^{i-2}(V) \rightarrow H^{i-3}(V)$ etc. are zero. Hence the cohomology of this complex vanishes and the $E_{2}$-term of the spectral sequence is zero.. Hence the spectral sequence abuts at $r=2$, which proves our claim.

To prove the injectivity for the first, third and so on differential $\bar{\partial}$ we use induction on $m$. For $m=1$ and $V=V\left(B e r^{i}\right) \in \mathcal{C}^{+}$we know $H_{D}(V)=0$ by theorem 4.1 and lemma 5.3. Since $H^{\nu}(V)=0$ for $\nu \neq i, i-1$, all higher differentials $d_{r}$ for $r \geq 2$ are zero by degree reasons. Hence $\bar{\partial}: H^{i}(V) \rightarrow$ $H^{i-1}(V)$ must be an isomorphism.

For the induction step put $V_{i}:=V(i, 1)$ and $N=V(i-2, m-1)$; then $V / N \cong V_{i}$. Hence we get a commutative diagram with horizontal exact sequences

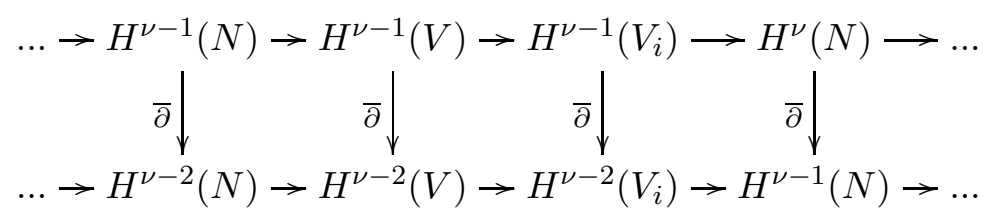

Since $H^{\nu}(N)=0$ for $\nu>i-2$ and $H^{\nu}\left(V_{i}\right)=0$ for $\nu \neq i, i-1$

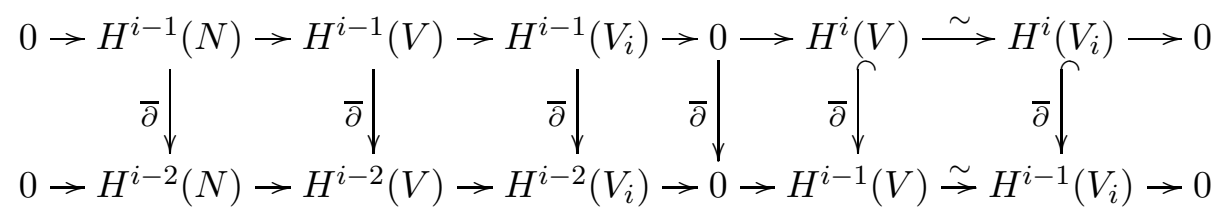

Thus $\bar{\partial}: H^{i}(V) \rightarrow H^{i-1}(V)$ is injective by a comparison with $V_{i}$. The assertion for the third, fifth and so on differential $\bar{\partial}$ follows from the induction assumption on $N$, since $H^{\nu}(V) \cong H^{\nu}(N)$ for $\nu \leq i-2$. 


\section{HodGE DECOMPOSITION}

We show in proposition 7.1 that the groups $H_{D}^{ \pm}(V)$ satisfy a Hodge decomposition. Put $F_{p}=F^{-p}$. This defines a decreasing filtration of $G_{n-1^{-}}$ modules $F_{p}\left(H_{D}^{ \pm}(V)\right)$ on $H_{D}^{ \pm}(V)$ as in the last section for $V \in T_{n}$. Here

$$
F_{p}\left(H_{D}^{ \pm}(V)\right)=\operatorname{Im}\left(\left(\bigoplus_{\ell \leq 2 p} M_{\ell}^{ \pm}\right) \cap \operatorname{Ker}(D) \rightarrow H_{D}^{ \pm}(V)\right) .
$$

One has also a decreasing filtration of $G_{n-1}$-modules $\bar{F}_{q}\left(H_{D}^{ \pm}(V)\right)$ on $H_{D}^{ \pm}(V)$ defined by the second filtration of the cohomology of $(\operatorname{Tot}(K), D)$ for the double complex $K^{\bullet \bullet}$ defined in the last section. It is defined by the subcomplexes $\bar{F}^{q}\left(\operatorname{Tot}(K)^{n}\right)=\bigoplus_{r+s=n, s \geq q} K^{r, s}$ of $(\operatorname{Tot}(K), D)$. Notice that

$$
\bar{F}^{q}\left(H_{D}^{ \pm}(V)\right)
$$

is the image of the $D$-cohomology of this subcomplex in $H_{D}(K)$. This filtration has analogous properties. In particular

$$
\bar{H}_{D}^{n-2 q}(V):=\bar{F}^{q}\left(\operatorname{Tot}^{n}(K)\right) / \bar{F}^{q+1}\left(\operatorname{Tot}^{n}(K)\right)
$$

by an analog of proposition 6.3 is isomorphic to

$$
\bar{H}_{D}^{\ell}(V) \cong H_{\partial}\left(\bar{H}^{\ell}(V)\right)
$$

where $\bar{H}^{\ell}(V)$ is defined as $H^{\ell}(V)$, only by using $\bar{\partial}$ instead of $\partial$.

We remark that both filtrations are functorial with respect to morphisms $f: V \rightarrow W$ in $T_{n}$. Hence also $V \mapsto \bar{F}^{q}\left(H_{D}^{n}(V)\right) \cap F_{p}\left(H_{D}^{n}(V)\right)$ defines a functor from $T_{n}$ to $T_{n-1}$.

Proposition 7.1. For all objects $V$ in $T_{n}$ we have a canonical decomposition of $H_{D}^{ \pm}(V)$ into $G_{n-1}$-modules

$$
H_{D}^{ \pm}(V)=\bigoplus_{\nu \in \mathbb{Z}} H_{D}^{\nu}(V)
$$

where for $\varepsilon=(-1)^{\nu}$

$$
H_{D}^{\nu}(V):=F_{\nu}\left(H_{D}^{\varepsilon}(V)\right) \cap \bar{F}_{\nu}\left(H_{D}^{\varepsilon}(V)\right) .
$$

Furthermore for $\mu>\nu$ we have

$$
F_{\nu}\left(H_{D}^{ \pm}(V)\right) \cap \bar{F}_{\mu}\left(H_{D}^{ \pm}(V)\right)=0 .
$$

Corollary 7.2. For a short exact sequence $0 \rightarrow A \rightarrow B \rightarrow C \rightarrow 0$ in $T_{n}$ the sequences

$$
H_{D}^{\nu}(A) \rightarrow H_{D}^{\nu}(B) \rightarrow H_{D}^{\nu}(C)
$$

are exact for all $\nu$. 
Remark. As shown after lemma 28.2 these halfexact sequences can not be extended to long exact sequences!

Proof. If $x \in H_{D}^{\nu}(B)$ maps to zero in $H_{D}^{\nu}(C) \subset H_{D}(C)$ there exist $y \in$ $H_{D}(A)$ such that $x$ is the image of $y$ by the exact hexagon for $H_{D}^{ \pm}$. But then, for the decomposition $y=\sum_{\nu} y_{\nu}$ and $y_{\nu} \in H_{D}^{\nu}(C)$ given in proposition 7, the components $y_{\nu}$ also maps to $x$ by the functoriality of $H_{D}^{\nu}($.$) .$

Proof of proposition 7.1. As in the proof of proposition 6.3 we can reduce to the case of an indecomposable object $V$ in $\mathcal{R}_{1}^{1}$. For such $V$ either $H_{D}(V)=0$, in which case the assertion is trivial, or $V$ is of the form

$$
0 \rightarrow L \rightarrow V \rightarrow Q \rightarrow 0
$$

with irreducible $L$ and $Q \in \mathcal{C}^{-}$or of the form

$$
0 \rightarrow U \rightarrow V \rightarrow L \rightarrow 0
$$

with irreducible $L$ and $U \in \mathcal{C}^{-}$. These two situation are duals of each other. So we restrict ourselves to the first case. The irreducible module $L$ is isomorphic to $\mathrm{Ber}^{m}$ for some $m \in \mathbb{Z}$. Then according to [Ger98] the quotient module $Q$ has socle and cosocle

$$
\begin{gathered}
\operatorname{socle}(Q)=\bigoplus_{i=1}^{s} \operatorname{Ber}^{m+2 i} \\
\operatorname{cosocle}(Q)=\bigoplus_{i=1}^{s} B e r^{m+2 i-1} .
\end{gathered}
$$

Recall $H^{m}(V)=H^{\bullet}(V)$, and hence $H_{D}(V)=H^{m}(V)$. Hence by the abutment of the spectral sequences

$$
H_{\bar{\partial}}\left(H^{\nu}(V)\right)=H^{\nu}(V) \cong k
$$

for $\nu=m$ and is zero otherwise. Hence $H^{m}(V) \cong H_{D}(V)$, since the filtration $F^{q}$ only jumps for $p=m$. Similarly

$$
H_{\partial}\left(\bar{H}^{\nu}(V)\right)=\bar{H}^{\nu}(V) \cong k
$$

for $\nu=m$ and is zero otherwise. Hence $\bar{H}^{m}(V) \cong H_{D}(V)$, since the filtration $\bar{F}^{q}$ only jumps for $q=m$. This simultaneous jump shows

$$
H_{D}^{m}(V)=\bar{F}^{m}\left(H_{D}(V)\right) \cap F_{m}\left(H_{D}(V)\right)
$$

and also for $q>p$.

$$
\bar{F}^{q}\left(H_{D}(V)\right) \cap F_{p}\left(H_{D}(V)\right)=0 .
$$




\section{THE CASE $m>1$}

As the diligent reader may have observed, the results obtained in the last sections on the functor $D S$ carry over to the case of the more general functors $D S_{n, n-m}$. For this fix $m \geq 1$. The enriched weight structure of $D S_{n, n-m}$ (which depends on $m$ ) is obtained from the decomposition of $(V, \rho) \in T_{n}$ into eigenspaces with respect to the eigenvalues $t^{\ell}$ under the elements $\varphi_{n, m}\left(E \times \operatorname{diag}\left(1, t^{-1}\right)\right)$ of the small torus. This allows to give a decomposition

$$
D S_{n, n-m}(V)=\bigoplus_{\ell} D S_{n, n-m}^{\ell}(V)[-\ell]
$$

into eigenspaces $\Pi^{\ell}\left(D S_{n, n-m}^{\ell}(V)\right)$ and gives long exact sequences in $T_{n-m}$ attached to short exact sequences in $T_{n}$ as in section 2 . Furthermore lemma 3.1 and lemma 3.2 carry over verbatim. Notice,

$$
D S_{n, n-m}^{\ell}\left(\operatorname{Ber}_{n}\right)=\operatorname{Ber}_{n-m} \quad, \quad \text { for } \ell=m
$$

and it is zero for $\ell \neq m$. Indeed, $\varphi_{n, m}\left(E \times \operatorname{diag}\left(1, t^{-1}\right)\right)$ acts on $B e r_{n}$ by $t^{m}$. Since $\epsilon_{n}=\varphi_{n, m}(E \times \operatorname{diag}(1,-1)) \epsilon_{n-m}$, the restriction of $B e r_{n}$ to $G_{n-m}$ via $\varphi_{n, m}$ defines the module $\Pi^{m}\left(\operatorname{Ber}_{m-n}\right)$.

Remark. Note that $D S_{n, n-m}^{\ell}(V)[-\ell]=\left(D S_{n, n-m}(V)\right)_{\ell}$. Here upper indices denote graduations without twist, lower indices graduations with twist. This is consistent with $D S_{n, n-1}^{\ell}(V)=H^{\ell}(V)$ in section 3. Note that it is essential to have non twisted objects such as $H^{\bullet}(V)$ or more generally $D S \bullet(V)$ due to the comparison with $H_{D}$ respectively $\omega_{n, n-m}$ (see below) which don't have any twists.

For $n-m_{1}=n_{1}$ and $n_{1}-m_{2}=n_{2}$ the functors $D S_{n, n_{1}}: T_{n} \rightarrow T_{n_{1}}$ and $D S_{n_{1}, n_{2}}: T_{n_{1}} \rightarrow T_{n_{2}}$ are related to the functor $D S_{n, n_{2}}: T_{n} \rightarrow T_{n_{2}}$ by a Leray type spectral sequence with the $E_{2}$-terms

$$
\bigoplus_{p+q=k} D S_{n_{1}, n_{2}}^{p}\left(D S_{n, n_{1}}^{q}(V)\right) \Longrightarrow D S_{n, n_{2}}^{k}(V) \text {. }
$$

To be more precise, choose matrices

$$
J=\left(\begin{array}{cc}
0 & J_{2} \\
J_{1} & 0
\end{array}\right)
$$

and $m_{i} \times m_{i}$-matrices $J_{i}, i=1,2$ with zero enties except for the entries 1 in the antidiagonal. Then $J$ and $J_{1}$ define functors $D S_{n, n_{2}}(V, \rho)=(V, \rho)_{x}$ resp. $D S_{n, n_{1}}(V, \rho)=(V, \rho)_{x_{1}}$ and $J_{2}$ defines a functor $D S_{n_{1}, n_{2}}(W, \psi)=(W, \psi)_{x_{2}}$. Obviously we have $x=x_{1}+x_{2} \in \mathfrak{g}_{n}$ and $x_{2} \in \mathfrak{g}_{n_{1}} \subset \mathfrak{g}_{n}$ such that $\left[x_{1}, x_{2}\right]=0$. 
Then indeed $\partial=\partial_{1}+\partial_{2}$ and $\partial_{1} \partial_{2}+\partial_{2} \partial_{1}=0$ for $\partial=\rho(x), \partial_{1}=\rho\left(x_{1}\right)$ and $\partial_{2}=\psi\left(x_{2}\right)$. Consider the weight (eigenvalue) decomposition

$$
V=\bigoplus_{p, q \in \mathbb{Z}} V^{p, q}
$$

of $(V, \rho)$ with respect to the matrices

$$
g\left(t_{1}, t_{2}\right)=\operatorname{diag}\left(1, . ., 1 ; t_{1}^{-1}, \ldots, t_{1}^{-1}, t_{2}^{-1}, . ., t_{2}^{-1}, 1, . ., 1\right)
$$

in $G_{n}\left(m_{1}\right.$ entries $t_{1}^{-1}$ and $m_{2}$ entries $\left.t_{2}^{-1}\right)$ so that $v \in V^{p, q}$ if and only if $g\left(t_{1}, t_{2}\right) v=t_{1}^{q} t_{2}^{p} \cdot v$. (We now write indices on top to avoid confusion with the lower indices $n$ and $n-m$ ). Then $\partial_{2}: V^{p, q} \rightarrow V^{p+1, q}$ and $\partial_{1}: V^{p, q} \rightarrow V^{p, q+1}$. Hence the Leray type spectral sequence is obtained by the spectral sequence of this double complex. For this note that the the functors $D_{n, n_{2}}^{k}$ are defined by the eigenvalues $t^{k}$ of the elements $g(t, t)$.

Proposition 8.1. For irreducible maximal atypical objects $L$ in $T_{n}$ the Leray type spectral sequence degenerates:

$$
D S_{n, n_{2}}(L) \cong D S_{n_{1}, n_{2}}\left(D S_{n, n_{1}}(L)\right) \text {. }
$$

Proof. Up to a parity shift, we can replace $L=L(\lambda)$ by $X_{\lambda}$ in $T_{n}$, so that $\operatorname{sdim}\left(X_{\lambda}\right)>0$ using that $\operatorname{sdim}(X) \neq 0$ [Ser10], [Wei10]. Then it suffices to prove inductively (for $D S$ applied $m$ times)

$$
(D S \circ D S \ldots \circ D S)\left(X_{\lambda}\right) \cong D S_{n, n-m}\left(X_{\lambda}\right) .
$$

The case $m=1$ is obvious by definition, since $D S_{n, n-1}=D S$. Suppose this assertion holds for $m$. Let us show that it then also holds for $m$ replaced by $m+1$. Indeed, the $E_{2}$-term of the spectral sequence

$$
D S \circ(D S \circ D S \ldots \circ D S)\left(X_{\lambda}\right) \Longrightarrow D_{n, n-m-1}\left(X_{\lambda}\right)
$$

are of the form

$$
D S \circ(D S \circ D S \ldots \circ D S)\left(X_{\lambda}\right) \cong \bigoplus_{\mu} X_{\mu}
$$

for irreducible representations $X_{\mu}$ in $T_{n-m-1}$ of superdimension $\operatorname{sim}\left(X_{\mu}\right)>$ 0 . Indeed this follows by repeatedly applying the later theorem 16.1, which implies $D S\left(X_{\lambda}\right) \cong \bigoplus_{i=1}^{k} X_{\lambda_{i}}$ for irreducible maximal atypical objects $X_{\lambda_{i}}$ in $T_{n-1}$ with $\operatorname{sidm}\left(X_{\lambda_{i}}\right)>0$. Now $D S$ is a tensor functor, and hence preserves superdimensions. Hence $\operatorname{sdim}\left(X_{\lambda}\right)=\sum_{\mu} \operatorname{sdim}\left(X_{\mu}\right)$. If the spectral sequence would not degenerate at $E_{2}$-level, then the $E_{\infty}$-term is a proper subquotient of the semisimple $E_{2}$-term. Hence

$$
\operatorname{sdim}\left(D S_{n-m-1}\right)\left(X_{\lambda}\right)<\sum_{\mu} \operatorname{sdim}\left(X_{\mu}\right),
$$


since $\operatorname{sdim}\left(X_{\mu}\right)>0$. This would imply

$$
\operatorname{sdim}\left(D S_{n-m-1}\right)\left(X_{\lambda}\right)<\operatorname{sdim}\left(X_{\lambda}\right) .
$$

However this is a contradiction, since $D_{n-m-1}$ is a tensor functor and hence $\operatorname{sdim}\left(D S_{n-m-1}\left(X_{\lambda}\right)\right)=\operatorname{sdim}\left(X_{\lambda}\right)$. Hence the spectral sequence degenerates and $D S_{n, n_{2}}\left(X_{\lambda}\right)$ has a filtration with graded pieces that are computed by appropriate $D S_{n_{1}, n_{2}}^{p} D S_{n, n_{1}}^{q}\left(X_{\lambda}\right)$. In order to prove that $D S_{n, n_{2}}\left(X_{\lambda}\right)=$ $D S_{n_{1}, n_{2}}\left(D S_{n, n_{1}}\left(X_{\lambda}\right)\right)$ we show that this filtration splits and $D S_{n, n_{2}}\left(X_{\lambda}\right)$ is semisimple. This follows from the sign rules of the main theorem 16.1. Indeed for $X_{\lambda}$ with $\varepsilon(\lambda)=1$, the constituents of $D S\left(X_{\lambda}\right)$ in $\mathcal{R}_{n}$ have the same sign $\varepsilon=1$ and the constituents of $D S\left(X_{\lambda}\right)$ in $\Pi \mathcal{R}_{n}$ have sign $\varepsilon=-1$. Now use that $\operatorname{Ext}^{1}\left(\mathcal{R}_{n}, \Pi \mathcal{R}_{n}\right)=0$ by lemma and $\operatorname{Ext}^{1}(L(\lambda), L(\mu))=0$ if $\varepsilon(\lambda)=\varepsilon(\mu)$ by corollary 15.1. Hence there are no extensions between the constituents of $D S\left(X_{\lambda}\right)$. Repeated application of $D S$ gives again constituents which are either in $\mathcal{R}_{n}$ with sign $\varepsilon=1$ or constituents in $\Pi \mathcal{R}_{n}$ with sign $\varepsilon=-1$. Since the constituents of $D S_{n, n_{2}}(L)$ are given by the constituents of the graded pieces, the semisimplicity follows.

We have seen in the last proposition that the Leray type spectral sequence degenerates at the $E_{2}$-level for irreducible maximal atypical objects. Let $F^{p}$ be the decending first (or second) filtration of the total complex. Due to the degeneration we can make use of the following lemma.

Lemma 8.2. Suppose given a finite double complex $\left(K^{\bullet}, \bullet, d_{\text {hor }}, d_{\text {vert }}\right)$ with associated total complex $K^{\bullet}=\operatorname{Tot}\left(K^{\bullet \bullet \bullet}\right)$ and total differential d. Suppose the associated spectral sequence for the first (second) filtration degenerates at the $E_{2}$-level and suppose $x \in F^{p}\left(K^{\bullet}\right)$ is a boundary in $K^{\bullet}$. Then there exists $y \in F^{p-1}\left(K^{\bullet}\right)$ such that $x=d y$.

Proof. We can assume that $x=\sum_{i=p}^{\infty} x_{p, n-p}$ has fixed degree $n$. The spectral sequence degenerates at $E_{2}$ and $[x]=0$ in $F^{p} H^{n}\left(K^{\bullet}\right)$. Hence the class of $x$ in $G r^{p}\left(H^{n}\left(K^{\bullet}\right)\right)=H_{h o r}^{p}\left(H_{v e r t}^{n-p}\left(K^{\bullet \bullet \bullet}\right)\right)$ vanishes. In other words there exists $v \in K^{p, n-p-1}$ and $u \in K^{p-1, n-p}$ such that $d_{\text {vert }}(u)=0$ and such that $d_{\text {hor }}(u)+d_{\text {vert }}(v)=x_{p, q}$. Hence $x-d(u+v) \in F^{p+1}\left(K^{\bullet}\right)$ with $u+v \in F^{p-1}\left(K^{\bullet}\right)$ again is closed. Iterating this argument we conclude that for any $r$ large enough we find $y \in F^{p-1}\left(K^{\bullet}\right)$ such that $x-d y \in F^{r}\left(K^{\bullet}\right)$. If $r$ is large enough, then $F^{r}\left(K^{\bullet}\right)=0$ and hence the claim follows.

Dirac cohomology. Similarly the results of section 5 hold verbatim for $\partial=\rho(x)$ and $\bar{\partial}=c \cdot \rho(\bar{x})$ and $D=\partial+\bar{\partial}$. In particular, for a generator $z$ of the Lie algebra of the center of $G_{1}$, let $H$ denote its image $\varphi_{n, m}(z) \in \mathfrak{g}_{n}$. The $D$-cohomology of the fixed space $V^{H}$ then gives objects $\omega_{n, n-m}(V, \rho)$ so that so that

$$
\omega_{n, n-m}: T_{n} \rightarrow T_{n-m}
$$


defines a tensor functor generalizing $H_{D}=\omega_{n, n-1}$. Note that $\omega_{n, n-m}$ restricts to a tensor functor $\omega_{n, n-m}: \mathcal{R}_{n} \rightarrow \mathcal{R}_{n-m}$ unlike $D S_{n, n-m}$.

As in section 6 there is a spectral sequence that allows to define a filtration on $\omega_{n, n-m}(V)$ whose graded pieces are

$$
\omega_{n, n-m}^{\ell}(V) \cong H_{\bar{\partial}}\left(D S_{n, n-m}^{\ell}(V)\right) \text {. }
$$

This generalizes proposition 6.3. Furthermore the results of proposition 7.1 and corollary 7.2 of section 7 carry over and define a Hodge decomposition for $\omega_{n, n-m}$ in terms of the functors $\omega_{n, n-m}^{\ell}$. Finally the same argument used in the proof of proposition 8.1 also shows

Proposition 8.3. For irreducible maximal atypical objects $L$ in $T_{n}$ the spectral sequence above degenerates, i.e. for all $\ell$

$$
\omega_{n, n-m}^{\ell}(L) \cong D S_{n, n-m}^{\ell}(L) \text {. }
$$

Now consider the $\mathbb{Z}$-graded object $D S_{n, n-m}^{\bullet}(L)=\bigoplus_{\ell \in \mathbf{Z}} D S_{n, n-m}^{\ell}(L)$ (which is different from $D S_{n, n-m}(L)$ if we forget the graduation) to compare with $\bigoplus_{\ell \in \mathbf{Z}} \omega_{n, n-m}^{\ell}(L)$ (that is $\omega_{n, n-m}(L)$ after forgetting the graduation).

Lemma 8.4. Suppose for irreducible $V \in T_{n}$ that $D S_{n, n_{1}}^{\bullet}(V) \cong \omega_{n, n_{1}}(V)$. Then

$$
\omega_{n, n_{2}}^{\bullet}(V) \cong \omega_{n_{1}, n_{2}}^{\bullet}\left(D S_{n, n_{1}}^{\bullet}(V)\right)
$$

holds.

Proof. Use that $\bar{\partial}=\bar{\partial}_{1}+\bar{\partial}_{2}$. By the assumption $D S_{n, n_{1}}^{\bullet}(V) \cong \omega_{n, n_{1}}^{\bullet}(V)$ the differential $\bar{\partial}_{1}$ is trivial on $D S_{n, n_{1}}^{\bullet}(V)$, hence trivial on $D S_{n_{1}, n_{2}}^{\bullet}\left(D S_{n, n_{1}}^{\bullet}(V)\right) \cong$ $D S_{n, n_{2}}^{\bullet}(V)$. Therefore the $\bar{\partial}$-homology of $D S_{n, n_{2}}^{\bullet}(V)$ is the same as the $\bar{\partial}_{2}$ homology attached to $D S_{n_{1}, n_{2}}^{\bullet}\left(D S_{n, n_{1}}(V)\right)^{\bullet}$.

This implies $\omega_{n, n-m}^{\bullet}(L) \cong D S_{n, n-m}^{\bullet}(L)$ for any irreducible $L$ in $T_{n}$. We prove this by induction on $m$. For $m=1$ this follows from the fact that irreducible representations satisfy property T. Now we use $\omega_{n, n-m-1}^{\bullet}(L) \cong$ $\omega_{n-m, n-m-1}^{\bullet}\left(D S_{n, n-m}^{\bullet}(L)\right)$ from lemma 8.4. Since $D S_{n, n-m}^{\bullet}(L)$ is semisimple by proposition 8.1 (as iteration of $m$ times $D S^{\bullet}$ ), we have

$$
\omega_{n-m, n-m-1}^{\bullet}\left(D S_{n, n-m}^{\bullet}(L)\right)=D S^{\bullet}\left(D S_{n, n-m}^{\bullet}(L)\right)=D S_{n, n-m-1}^{\bullet}(L) .
$$

This implies

Proposition 8.5. For all irreducible objects $L$ in $T_{n}$ and all $\ell$ we have

$$
\omega_{n, n-m}^{\ell}(L) \cong D S_{n, n-m}^{\ell}(L) \text {. }
$$


The case $m=n$ is of particular interest. Notice that $T_{0}$ is the category $\operatorname{svec}_{k}$ of finite dimensional super $k$-vectorspaces. Hence

$$
\omega=\omega_{n, 0}: T_{n} \longrightarrow \operatorname{svec}_{k} .
$$

The tori $A_{i}$. Let $A_{i} \subseteq G_{n}$ denote the diagonal torus of all elements of the form $\operatorname{diag}\left(1, \ldots, 1, t_{n-i+1}, \ldots, t_{n} \mid t_{n}, \ldots, t_{n-i+1}, 1, \ldots, 1\right)$. In particular $A_{1}$ is the torus of section 5 and $H=H_{n, n-1}$. It commutes with all operators $\partial_{n, n-i}, \bar{\partial}_{n, n-i}$ and $D_{n-i}$ and hence acts on $D S_{n, n-i}(V)$ respectively $\omega_{n, n-i}(V)$. We claim

Lemma 8.6. The action of $A_{i}$ on $D S_{n, n-i}(V)$ and $\omega_{n, n-i}(V)$ is trivial.

Proof. For this we can assume without loss of generality that $i=n$. The $H_{n, n-i}$ for $i=1, \ldots, n$ generate the Lie algebra of the torus $A$. Hence it suffices that all $H_{n, n-i}$ act trivially. This follows from the Leray type spectral sequence $D S_{n-i, 0} \circ D S_{n, n-i} \Longrightarrow D S_{n, 0}$. As in the proof of lemma 5.1 one shows that $H_{n, n-i}$ acts trivially on $D S_{n, n-i}(V)$. Hence by the spectral sequence $H_{n, n-i}$ acts by a nilpotent matrix on $D_{n, 0}(V)$. On the other hand $A$, and hence $H_{n, n-i} \in \operatorname{Lie}(A)$, acts in a semisimple way. This proves the claim.

\section{BOUNDARY MAPS}

Suppose given a module $S$ in $\mathcal{R}_{n}$. Consider $D_{t o t}=D+D^{\prime}$ for $D=D_{n, n-i}$ and $D^{\prime}=D_{n-i, 0}$. Notice that $D D^{\prime}=-D^{\prime} D$ and $D^{2}=c \rho(H),\left(D^{\prime}\right)^{2}=$ $c \rho\left(H^{\prime}\right)$ and $D_{t o t}^{2}=c \rho\left(H_{t o t}\right)$.

For fixed $i$ we write $A=A_{i}$. We have $H_{D}(S)=\operatorname{Kern}(D: S \rightarrow S) /\left(S^{H} \cap\right.$ $\operatorname{Im}(D: S \rightarrow S))$. We have also shown that this is equal to

$$
H_{D}(S)=\operatorname{Kern}\left(D: S^{A} \rightarrow S^{A}\right) / \operatorname{Im}\left(D: S^{A} \rightarrow S^{A}\right)
$$

for the torus $A$ whose Lie algebra is generated by all $H_{n, n-j}$ for $j=1, \ldots, n$. In a similar way

$$
H_{D_{t o t}}(S)=\operatorname{Kern}\left(D_{t o t}: S^{A} \rightarrow S^{A}\right) / \operatorname{Im}\left(D_{t o t}: S^{A} \rightarrow S^{A}\right) .
$$

Recall that $A$ commutes with $D_{t o t}, D, D^{\prime}$ and acts in a semisimple way.

Let $U \subseteq S$ denote the image of $D^{\prime}: S^{A} \rightarrow S^{A}$. Then $U$ and $\bar{S}^{A}=S^{A} / U$ are stable under $D$ and $D^{\prime}$. If $s \in S^{A}$ is in $\operatorname{Kern}\left(D_{t o t}\right)$, then $D s=-D^{\prime} s \in U$. Hence $s \mapsto s+U$ defines a map from $\operatorname{Kern}\left(D_{t o t}: S^{A} \rightarrow S^{A}\right) / \operatorname{Im}\left(D_{\text {tot }}\right.$ : $\left.S^{A} \rightarrow S^{A}\right)$ to $\operatorname{Kern}\left(D: \bar{S}^{A} \rightarrow \bar{S}^{A}\right) / \operatorname{Im}\left(D_{t o t}: \bar{S}^{A} \rightarrow \bar{S}^{A}\right)$, hence a map or

$$
\sigma_{S}: H_{D_{t o t}}(S) \longrightarrow H_{D}(\bar{S}) \text {. }
$$


Suppose given modules $S, V, L$ in $\mathcal{R}_{n}$ defining an extension

$$
0 \rightarrow S \rightarrow V \rightarrow L \rightarrow 0 \text {. }
$$

We get a boundary map

$$
\delta_{t o t}: H_{D_{t o t}}^{ \pm}(L) \longrightarrow H_{D_{t o t}}^{\mp}(S)
$$

defined as usually by $\operatorname{Kern}\left(D_{t o t}: L \rightarrow L\right) \ni \bar{v} \mapsto[s], s=D_{t o t} v \in L$. Here $v \in V^{A}$ is any lift of $\bar{v} \in L^{A}$ (it exists by the semisimple action of $A$ on $V$ ). Obviously $D_{\text {tot }}(s)=0$, since $D_{\text {tot }}^{2}=0$ on the space of $A$-invariant vectors. Therefore the class $[s]$ of $s$ in $H_{D_{t o t}}^{\mp}(S)$ is well defined.

In a completely similar way one defines the boundary map

$$
\delta: H_{D}^{ \pm}(L) \longrightarrow H_{D}^{\mp}(S) .
$$

We claim that there exists a commutative diagram

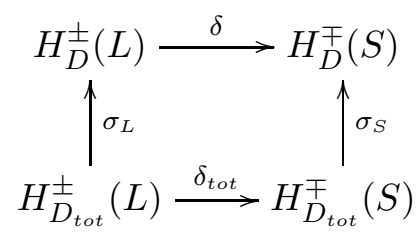

In fact on the level of representatives $v \in V^{A}$ it amounts to the assertion

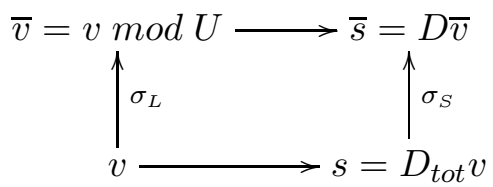

using $D_{t o t} v \equiv D v \bmod U_{L}$.

We now consider two extension $(S, V, L)$ and $(S, \tilde{V}, \tilde{L})$. Then the commutative diagram

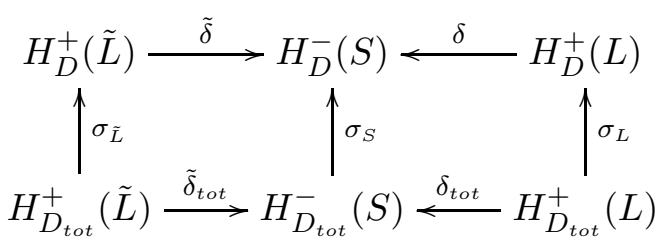

implies

Lemma 9.1. Suppose $L \cong 1$. Then $\operatorname{Im}\left(\delta_{t o t}\right)$ is not contained in $\operatorname{Im}\left(\tilde{\delta}_{t o t}\right)$, if there exists an integer $i$ for $1 \leq i \leq n$ such that

- $H_{D}^{+}(\tilde{L})$ does not contain 1 as a $G_{n-i}$-module.

- $\delta(\mathbf{1}) \neq 0$ in $H_{D}^{-}(S)$.

for $D=D_{n, n-i}$. 
Remark. If $S=L_{n}(j)$ for some $1 \leq j \leq n$ is one of the hook representation discussed in section 31, then the smallest integer $i$ for which $\delta(\mathbf{1}) \neq 0$ in $H_{D}^{-}(S)$ holds is given by $n-i=j-1$. Indeed we later show that although $0 \rightarrow D_{n, j}^{0}(S) \rightarrow D_{n, j}^{0}(V) \rightarrow \mathbf{1} \rightarrow 0$ is exact, the map $D_{n, j-1}^{0}(V) \rightarrow \mathbf{1}$ is not surjective any longer. So in the later applications, to apply the last lemma, we have to check whether $H_{D}^{+}(\tilde{L})$ contains the trivial $G_{j-1}$-module in this case or not.

\section{Highest Weight Modules}

Irreducible representations. The irreducible $G l(n \mid n)$-modules $L$ in $\mathcal{R}_{n}$ are uniquely determined up to isomorphism by their highest weights $\lambda$. These highest weights $\lambda$ are in the set $X^{+}(n)$ of dominant weights, where $\lambda$ is in $X^{+}(n)$ if and only if $\lambda$ is of the form

$$
\lambda=\left(\lambda_{1}, \lambda_{2}, \ldots, \lambda_{n} ; \lambda_{n+1}, \ldots, \lambda_{2 n}\right)
$$

with integers $\lambda_{1} \geq \lambda_{2} \geq \ldots \geq \lambda_{n}$ and $\lambda_{n+1} \geq \lambda_{n+2} \geq \ldots \geq \lambda_{2 n}$.

We remark that the condition

$$
\lambda_{n}=-\lambda_{n+1}
$$

for $\lambda$ is equivalent to the condition $\lambda(H)=0$. In the language of Brundan and Stroppel in section 12 the condition $\lambda(H)=0$ is tantamount to the condition that the irreducible representation $L(\lambda)$ is not projective and the smallest $\vee$-hook is to the left of all $\times$ 's and o's. Any at least 1-atypical block contains such $L(\lambda)$. If these equivalent conditions hold we write

$$
\bar{\lambda}=\left(\lambda_{1}, \ldots, \lambda_{n-1} ; \lambda_{n+2}, \ldots, \lambda_{2 n}\right)
$$

defining an irreducible representation $L(\bar{\lambda})$ in $\mathcal{R}_{n-1}$.

Using the notation of [Dro09] the irreducible maximally atypical $G l(n \mid n)$ modules $L$ in $\mathcal{R}_{n}$ are given by highest weights $\lambda$ of the form

$$
\lambda=\left(\lambda_{1}, \lambda_{2}, \ldots, \lambda_{n} ;-\lambda_{n}, \ldots,-\lambda_{1}\right)
$$

with integers $\lambda_{1} \geq \lambda_{2} \geq \ldots \geq \lambda_{n}$. We abbreviate this by writing $\left[\lambda_{1}, \ldots, \lambda_{n}\right]$ for the corresponding irreducible representation. The full subcategory of $\mathcal{R}_{n}$ generated by these will be denoted $\mathcal{R}_{n}^{n}$.

Highest weight modules. Recall that a vector $v \neq 0$ in a module $(V, \rho)$ in $\mathcal{R}_{n}$ is called primitive, if $\rho(X) v=0$ holds for all $X$ in the standard Borel subalgebra $\mathfrak{b}$ of $\mathfrak{g}=\mathfrak{g}_{n}$. A highest weight vector of a module $V$ (of weight $\lambda$ ) in $\mathcal{R}_{n}$ is a vector $v \in V$ that is a primitive eigenvector of $\mathfrak{b}$ (of the weight $\lambda$ ) generating the module $V$. In this case $V$ is called a highest weight module (of weight $\lambda$ ). Every irreducible representation $L(\lambda)$ in $\mathcal{R}_{n}$ is a highest 
weight module of weight $\lambda$. Every highest weight module $V$ of weight $\lambda$ has cosocle isomorphic to $L(\lambda)$.

Lemma 10.1. For $(V, \rho)=L(\lambda)$, or more generally a cyclic representation generated by a highest weight vector of weight $\lambda$, the weight space in $V$ of weight $\lambda-\mu$ is generated by $\rho(\bar{x}) v$, where $v$ is a highest weight vector of $(V, \rho)$.

Proof. For the simple positive roots $\Delta=\left\{\alpha_{1}, \ldots, \alpha_{r}\right\}$, i.e. the union of the odd simple root $\{\mu\}$ and the even simple roots in $\mathfrak{g}_{0}$ with respect to the standard Borel subalgebra of upper triangular matrices, choose generators $X_{\alpha} \in \mathfrak{u}$. Put $\tau\left(X_{\alpha}\right)=Y_{-\alpha}$ and $V_{0}=F \cdot v$. Recursively define $V_{i}=V_{i-1}+$ $\sum_{\alpha \in \Delta} \rho\left(Y_{-\alpha}\right)\left(V_{i-1}\right)$. We claim that $V_{\infty}=\bigcup_{i=0}^{\infty} V_{i}$ is a $\mathfrak{g}$-submodule of $V$, hence equal to $V$. This claim also implies that the weight space $V_{\lambda-\mu}$ is generated by $\rho(\tau(x)) v$.

$V_{\infty}$ is invariant under all $\rho\left(Y_{-\alpha}\right), \alpha \in \Delta=\left\{\alpha_{1}, \ldots, \alpha_{r}\right\}$. Each $V_{i}$ obviously is invariant under $\rho(X)$ for diagonal $X \in \mathfrak{g}$. Indeed each $V_{i} / V_{i-1}$ decomposes in weight spaces for weights

$$
\lambda-\sum_{j=1}^{r} n_{j} \alpha_{j} \quad, \quad \sum_{j=1}^{r} n_{j}=i \quad\left(n_{j} \in \mathbb{N}_{\geq 0}\right) .
$$

Note $\rho\left(X_{\alpha}\right) \rho\left(Y_{-\beta}\right) \pm \rho\left(Y_{-\beta}\right) \rho\left(X_{\alpha}\right)=\rho\left(H_{\alpha}\right)$ for $\alpha=\beta \in \Delta$ and $\rho\left(X_{\alpha}\right) \rho\left(Y_{-\beta}\right) \pm$ $\rho\left(Y_{-\beta}\right) \rho\left(X_{\alpha}\right)=\rho\left(\left[X_{\alpha}, Y_{-\beta}\right]\right)=0$ for $\alpha, \beta \in \Delta$ and $\alpha \neq \beta$ [since $\alpha-\beta \notin$ $\Phi^{+} \cup \Phi^{-}$for $\left.\alpha, \beta \in \Delta\right]$. Hence $V_{\infty}$ is invariant under $g$, since $Y_{-\beta}, \beta \in \Delta$ and diagonal $X$ and $X_{\alpha}, \alpha \in \Delta$, generate $\mathfrak{g}$ as a Lie superalgebra.

Lemma 10.2. Suppose $\lambda=\left(\lambda_{1}, \ldots, \lambda_{n-1}, \lambda_{n} ; \lambda_{n+1}, \lambda_{n+2}, \ldots, \lambda_{2 n}\right)$ satisfies $\lambda_{n}=-\lambda_{n+1}$. If $V$ is a highest weight representation generated by a highest weight vector $v$ of weight $\lambda$, the module $H^{\lambda_{n}}(V)$ contains a highest weight submodule of weight $\bar{\lambda}$ generated by the image of $v$ with parity $(-1)^{\lambda_{n}}$. In particular the representation $\Pi^{\lambda_{n}} L(\bar{\lambda})$ in $\mathcal{R}_{n-1}$ is a JordanHölder constituent of $H^{\lambda_{n}}(V)$.

Proof of the lemma. The highest weight vector $v$ of $V$ is a highest weight vector of the restriction of $V$ to the subgroup $G_{n-1}$ of $G_{n}$ and is annihilated by $\rho(x)$. By our assumption on the weight $\lambda$ furthermore $v \in V^{H}$. To prove our claim it suffices to show that $v$ is not contained in $\operatorname{Im}(\rho(x))$. Suppose $v=\rho(x)(w)$. Since the weight of $x$ is $\mu$, we can assume that the weight of $w$ is $\lambda-\mu$. Since $V$ is a highest weight representation, by lemma 10.1 then $w$ is proportional to $\rho(\bar{x}) v$. So that to show $\rho(x) w=0$ and to finish our proof, it suffices that by $[x, \bar{x}]=H$

$$
\rho(x) \rho(\bar{x}) v=-\rho(\bar{x}) \rho(x) v+\rho(H) v=0
$$


vanishes, since $\rho(x) v=0$ and $v \in V^{H}$.

Note that (for the notation used see section 11)

$$
z_{i}=\left[x_{i}, \bar{x}\right]=x_{i} \bar{x}+\bar{x} x_{i} \quad, \quad z_{i}^{\prime}=\left[x_{i}^{\prime}, \bar{x}\right]=x_{i}^{\prime} \bar{x}+\bar{x} x_{i}^{\prime}
$$

are in the unipotent Lie algebra $\mathfrak{u}_{\overline{0}} \subset \mathfrak{u}$ of the standard Borel $\mathfrak{b}_{\overline{0}}$ of $\mathfrak{g}_{\overline{0}}$ for all $i=1, . ., n-1$. Suppose $(V, \rho)$ is a representation of $G_{n}$. If $(V, \rho)$ has a highest weight vector $v$, then $\rho(X) v=0$ holds for all $X$ in the unipotent radical $\mathfrak{u}$ of the standard Borel of $\mathfrak{g}$. In particular

$$
\rho(x) v=0, \rho\left(x_{i}\right) v=0, \rho\left(x_{i}^{\prime}\right) v=0, \rho\left(z_{i}\right) v=0, \rho\left(z_{i}^{\prime}\right) v=0
$$

and hence by the commutation relations above this implies for $i=1, \ldots, n-1$ also

$$
\rho\left(x_{i}\right) \rho(\bar{x}) v=0 \quad, \quad \rho\left(x_{i}^{\prime}\right) \rho(\bar{x}) v=0 .
$$

Now also suppose $v \in V^{H}$ and put $w=\rho(\bar{x}) v$. Then $\rho(x) w=0$, as shown in the proof of lemma 10.2. Similarly one can show $\rho\left(x_{i}\right) w=0$ (since $\left.\rho\left(x_{i}\right) v=\rho\left(z_{i}\right) v=0\right)$ and $\rho\left(x_{i}^{\prime}\right) w=0$. All elements $\mathfrak{u} \cap \mathfrak{g}_{n-1}$ commute with $\rho(\bar{x})$ and annihilate $v$, hence annihilate $w$. Finally, since $\rho(\bar{x})$ and $\rho\left(x_{i}\right), \rho\left(x_{i}^{\prime}\right)$ annihilate $w$, also $\rho\left(z_{i}\right)$ and $\rho\left(z_{i}^{\prime}\right)$ annihilate $w$. It follows that $\rho(X) w=0$ for all $X \in \mathfrak{u}$, since $\mathfrak{u}$ is spanned by $\mathfrak{u} \cap \mathfrak{g}_{n-1}$ and the $x, x_{i}, x_{i}^{\prime}, z_{i}, z_{i}^{\prime}$,. This implies that $w$ is a highest weight vector in $(V, \rho)$ of weight $\lambda-\mu$, if $w \neq 0$. Hence

Corollary 10.3. If $(V, \rho)$ is a highest weight representation with highest weight vector $v$ and highest weight $\lambda$ so that $\lambda(H)=0$, then $w=\rho(\bar{x}) v$ defines a highest weight vector of weight $\lambda-\mu$ in $V$ if $w \neq 0$.

In the situation of the last corollary, the following conditions are equivalent

(1) $w=0$

(2) $D(v)=0$

(3) $D(v)=0$ and $v$ defines a nonvanishing cohomology class in $H_{D}(V)$. Indeed $D(v)=i \rho(\bar{x}) v+\rho(x) v=i w$. Furthermore, if $v=D(\tilde{w})$, then $v=$ $i \rho(\bar{x}) \tilde{w}_{1}+\rho(x) \tilde{w}_{2}$ for $w_{1} \in V_{\lambda+\mu}$ and $w_{2} \in V_{\lambda-\mu}$. Since $\lambda$ is highest weight, therefore $V_{\lambda+\mu}=0$. Furthermore $V_{\lambda-\mu}$ is generated by $w$, and $\rho(x) w=0$. Hence $v \notin D(V)$.

A highest weight representation $V$ of weight $\lambda$ canonically admits the irreducible representation $L=L(\lambda)$ as a quotient. Let $q: V \rightarrow L$ denote the quotient map.

Corollary 10.4. In the highest weight situation of corollary 10.3 the following holds for the representation $V$ : 
(1) If $V$ contains a highest weight subrepresentation $W \neq 0$ of weight $\lambda-\mu$, then $H_{D}^{\lambda_{n}}(V)$ has trivial weight space $H_{D}^{\lambda_{n}}(V)_{\bar{\lambda}} \subseteq H_{D}^{\lambda_{n}}(V)$.

(2) If the natural map $H_{D}(q): H_{D}(V) \rightarrow H_{D}(L)$ is surjective, then $V$ does not contain a highest weight subrepresentation $W \neq 0$ of weight $\lambda-\mu$.

Proof. For the first assertion, notice that $D(v)=0$ implies $w=0$ and $w$ generates $V_{\lambda-\mu}$. For the second assertion notice that the highest weight vector $v \in V$ maps to the highest weight vector $q(v)$ of $L$. By the first assertion and lemma 10.2, applied for $L$, the vector $q(v)$ is $D$-closed and defines a nonzero class in $H_{D}^{\lambda_{n}}(L)_{\bar{\lambda}}$. Since now $H_{D}(q)$ is surjective by assumption, corollary 7.2 implies that this class is the image of a nonzero cohomology class $\eta$ in $H_{D}^{\lambda_{n}}(V)$. This class is representated by a nonzero $\bar{\partial}$ closed class in $H^{\lambda_{n}}(V)=D S_{\lambda_{n}}$ in the weight space $\bar{\lambda}$. Hence this class has a $D$-closed representative $v^{\prime}$ in $V_{\lambda}$, since the enriched weight structure on $D S(V)$ allows to recover the weight structure of $V$. Since $V$ is a highest weight representation, the space $V_{\lambda}$ has dimension one and therefore $v^{\prime}$ is proportional to $v$. Thus $D(v)=0$. But, as explained above, this implies $w=0$ and hence $V_{\lambda-\mu}=0$.

Since Kac modules $V(\lambda)$ are highest weight modules of weight $\lambda$ with $H_{D}(V(\lambda))=0$, lemma 10.2 and its corollaries above imply

Lemma 10.5. For $\lambda$ in $X^{+}$with $\lambda_{n}=\lambda_{n+1}=0$ the cohomology $H^{0}(V(\lambda))$ of the Kac module $V(\lambda)$ contains a highest weight module of weight $\bar{\lambda}$. Furthermore $V(\lambda)$ contains a nontrivial highest weight representation of weight $\lambda-\mu$.

Example. Let $(V, \rho)=V(\mathbf{1})$ in $\mathcal{R}_{2}$ be the Kac module of the trivial representation. Then $D S\left(V^{*}\right)=0$ and $D S(V) \neq 0$, since $V$ is not projective. The module $V$ is a cyclic module generated by it highest weight vector of weight $\lambda=0$ (this is not true for the anti-Kac module $V^{*}$ ). Furthermore $V$ has Loewy length 3 with Loewy series $\left(B e r_{2}^{-2}, \operatorname{Ber}_{2}^{-1} S^{1}, \mathbf{1}\right)$ where $S^{1}=$ $[1,0]$. We claim

$$
D S(V)=\left(\operatorname{Ber}_{1}^{-2} \oplus \mathbf{1}\right) \otimes(\mathbf{1} \oplus \Pi(\mathbf{1})) .
$$

This follows from the later results, e.g. lemma 2.1 and theorem 16.1: $d\left(\operatorname{Ber}_{2}\right)=-\operatorname{Ber}_{1}$ and $d\left(S^{1}\right)=B e r_{1}^{-1}+\operatorname{Ber}_{1}$ imply $d(V)=0$, hence $D S(V)$ has at most 4 Jordan-Hölder constituents $B e r_{1}^{-2}, \Pi\left(B e r_{1}^{-2}\right), \mathbf{1}, \Pi(\mathbf{1})$. By lemma 10.2 the constituent 1 occurs. By duality then also the constituent $B e r_{1}^{-2}$ must occur. Since $d(V)=0$ the constituent $\Pi\left(B e r_{1}^{-2}\right), \Pi(\mathbf{1})$ must occur. Finally apply proposition 19.1. This example shows that $D S$ in general does not preserve negligible objects. 
Highest weights. Suppose $(V, \rho)$ is a highest weight module of weight $\lambda$ such that $\lambda(H)=0$. Let $\nu$ be a weight of $V$. Then

$$
\nu=\lambda-\sum_{\alpha \in \Delta_{n}} \mathbb{N}_{\geq 0} \cdot \alpha
$$

for the set $\Delta_{n}$ of simple positive roots $\alpha$ of $G_{n}$.

Now suppose $\nu$ contributes to $D S(V)$. Then $\nu$ is a weight of $V^{H}$ and hence $\nu(H)=0$. Notice $\Delta_{n}$ is the union of $\Delta_{n}^{+}=\left\{e_{1}-e_{2}, \ldots, e_{n-1}-\right.$ $\left.e_{n}, e_{n+1}-e_{n+2}, \ldots, e_{2 n-1}-e_{2 n}\right\}$ and $\Delta_{n}^{-}=\left\{e_{n}-e_{n+1}\right\}$. The restriction of the simple roots $\alpha \in \Delta_{n}$ are in $\Delta_{n-1}$ (i.e. simple root of $G_{n-1}$ ) except for the even simple roots $\alpha=e_{n-1}-e_{n}, \alpha=e_{n+1}-e_{n+2}$ and the odd simple root $\alpha=$ $e_{n}-e_{n-2}$. A linear combination $\sum_{\alpha \in \Delta_{n}} n_{\alpha} \alpha$ annihilates $H$ if and only if the coefficient, say $m$, of $e_{n-1}-e_{n}$ and $e_{n+1}-e_{n+2}$ coincides; hence this holds iff $\nu$ is of the form $\sum_{\alpha \in \Delta_{n-1}^{+}} n_{\alpha} \alpha+\left(n_{\mu}-m\right) \cdot\left(e_{n}-e_{n+1}\right)+m \cdot\left(e_{n-1}-e_{n+2}\right)$. Notice that $\mu=\left(e_{n}-e_{n+1}\right)$ is trivial on the maximal torus of $G_{n-1}$ and that $\left(e_{n-1}-e_{n+2}\right)$ defines the new odd simple root in $\Delta_{n-1}^{-}$. Hence the restriction of $\nu \in V^{H}$ is of the form

$$
\left.\left.\nu\right|_{\mathfrak{b} \cap \mathfrak{g}_{n-1}} \in \lambda\right|_{\mathfrak{b} \cap \mathfrak{g}_{n-1}}-\sum_{\alpha \in \Delta_{n-1}} \mathbb{N}_{\geq 0} \cdot \alpha
$$

under our assumptions above. Notice for $V_{\lambda} \subset V^{H} \subset V$ we have

$$
\ell=\lambda\left(\operatorname{diag}\left(1, . ., 1 ; t^{-1}, 1, . ., 1\right)\right)=n_{\mu}-m=\lambda_{n}^{\prime} .
$$

The discussion above implies

Lemma 10.6. For a highest weight module $(V, \rho)$ in $T_{n}$ of weight $\lambda$ with $\lambda(H)=0$ the module $D S(V, \rho)$ has its weights $\nu$ in $\lambda-\sum_{\alpha \in \Delta_{n-1}} \mathbb{N}_{\geq 0} \cdot \alpha$.

Corollary 10.7. Given $(V, \rho) \in T_{n}$, suppose $L(\lambda)$ is a Jordan-Hölder constituent of $(V, \rho)$ such that for all Jordan-Hölder constituents $L(\nu)$ of $(V, \rho)$ we have $\nu \in \lambda-\sum_{\alpha \in \Delta_{n}} \mathbb{N}_{\geq 0} \cdot \alpha$ and $\nu(H)=0$. Then $L(\bar{\lambda})$ appears in $D S(V, \rho)$ and all other irreducible constituents $L\left(\nu^{\prime}\right)$ or $\Pi L\left(\nu^{\prime}\right)$ of $D S(V, \rho)$ satisfy $\nu^{\prime} \in \lambda-\sum_{\alpha \in \Delta_{n-1}} \mathbb{N}_{\geq 0} \cdot \alpha$.

Proof. This follows from the last lemma and the weak exactness of the functor $D S$.

\section{THE CASIMIR}

We study the operation of the Casimir $C_{n}$ on $D S(V)$. This will be used in section 18 when we study the effect of $D S$ on translation functors $F_{i}\left(L_{\times \circ}\right)$. 
Consider the fixed element $x \in \mathfrak{g}_{n}$

$$
x=\left(\begin{array}{cc}
0 & y \\
0 & 0
\end{array}\right) \in \mathfrak{g}_{n} \text { for } y=\left(\begin{array}{cccc}
0 & 0 & \ldots & 0 \\
0 & 0 & \ldots & 0 \\
\ldots & & \ldots & \\
1 & 0 & 0 & 0
\end{array}\right)
$$

Similarly we define

$$
x_{i}, x_{i}^{\prime} \text { for } i=1, . ., n-1
$$

for matrices $y=y_{i}$ resp. $y_{i}^{\prime}$ with a unique entry 1 in the first column resp. last row at positions different from the entry 1 in the above $y$

$$
\left(\begin{array}{cccc}
* & 0 & \ldots & 0 \\
* & 0 & \ldots & 0 \\
\ldots & & \ldots & \\
0 & * & * & *
\end{array}\right)
$$

Then $x, x_{i}, x_{i}^{\prime}$ are in $u$ for $i=1, \ldots, n-1$. The elements $x_{i}, x_{i}^{\prime}$ satisfy $\left[x_{i}, x\right]=$ $0=\left[x_{i}^{\prime}, x\right]$.

Using Brundan-Stroppel's notations [BS12a], (2.14), let $e_{r, s} \in \mathfrak{g}_{n}$ be the $r s$-matrix unit. Then the Casimir operator $C_{n}=\sum_{r, s=1}^{n}(-1)^{\bar{s}} e_{r, s} e_{s, r}$ of the super Lie algebra $\mathfrak{g}_{n}=\operatorname{Lie}\left(G_{n}\right)$ is recursively given by

$$
\begin{gathered}
C_{n}=C_{n-1}+C_{1}+2\left(\bar{z}_{1} z_{1}+\cdots+\bar{z}_{n-1} z_{n-1}\right)+\left(e_{1,1}+\cdots+e_{n-1, n-1}-(n-1) e_{n, n}\right) \\
-2\left(\bar{z}_{1}^{\prime} z_{1}^{\prime}+\cdots+\bar{z}_{n-1}^{\prime} z_{n-1}^{\prime}\right)-\left(-e_{n+2, n+2}-\cdots-e_{2 n, 2 n}+(n-1) e_{n+1, n+1}\right) \\
+2\left(\bar{x}_{1} x_{1}+\cdots+\bar{x}_{n-1} x_{n-1}\right)-\left(e_{1,1}+\cdots+e_{n-1, n-1}+(n-1) e_{n+1, n+1}\right) \\
\quad+2\left(\bar{x}_{1}^{\prime} x_{1}^{\prime}+\cdots+\bar{x}_{n-1}^{\prime} x_{n-1}^{\prime}\right)-\left(e_{n+2, n+2}+\cdots+e_{2 n, 2 n}+(n-1) e_{n, n}\right)
\end{gathered}
$$

with the notations $x_{i}=e_{i, n+1}, x_{i}^{\prime}=e_{n, 2 n+1-i}, z_{i}=e_{i, n}$ and $z_{i}^{\prime}=e_{n+1,2 n+1-i}$. Furthermore $\bar{x}_{i}, \bar{x}_{i}^{\prime}, \bar{z}_{i}$ and $\bar{z}_{i}^{\prime}$ denote the supertransposed of $x_{i}, x_{i}^{\prime}, z_{i}$ and $z_{i}^{\prime}$. Hence

$$
\begin{gathered}
C_{n}=C_{n-1}+C_{1}+2\left(\bar{z}_{1} z_{1}+\cdots+\bar{z}_{n-1} z_{n-1}-\bar{z}_{1}^{\prime} z_{1}^{\prime}-\cdots-\bar{z}_{n-1}^{\prime} z_{n-1}^{\prime}\right) \\
+2\left(\bar{x}_{1} x_{1}+\cdots+\bar{x}_{n-1} x_{n-1}+\bar{x}_{1}^{\prime} x_{1}^{\prime}+\cdots+\bar{x}_{n-1}^{\prime} x_{n-1}^{\prime}\right)-2(n-1) H
\end{gathered}
$$

using $\left[\tau(x), x_{i}\right]=z_{i}$ and $\left[\tau(x), x_{i}^{\prime}\right]=z_{i}^{\prime}$ and

$$
\left[z_{i}, \bar{z}_{i}\right]=e_{i, i}-e_{n, n} \quad, \quad\left[z_{i}^{\prime}, \bar{z}_{i}^{\prime}\right]=e_{n+1, n+1}-e_{2 n+1-i, 2 n+1-i}
$$

and $\left[\bar{x}_{i}, x_{i}\right]=e_{i, i}+e_{n+1, n+1}$ and $\left[\bar{x}_{i}^{\prime}, x_{i}^{\prime}\right]=e_{2 n+1-i, 2 n+1-i}+e_{n, n}$. Notice $\bar{x}_{i} x_{i}-$ $x_{i} \bar{x}_{i}=2 \bar{x}_{i} x_{i}-e_{i, i}-e_{n+1, n+1}$ and $\bar{x}_{i}^{\prime} x_{i}^{\prime}-x_{i}^{\prime} \bar{x}_{i}^{\prime}=2 \bar{x}_{i}^{\prime} x_{i}^{\prime}-e_{2 n+1-i, 2 n+1-i}-e_{n, n}$. Finally $C_{1}=e_{n, n}^{2}-e_{n+1, n+1}^{2}-x \bar{x}+\bar{x} x=e_{n, n}^{2}-e_{n+1, n+1}^{2}+2 \bar{x} x-H$.

Representations. Suppose $(V, \rho)$ is a representation of $\mathfrak{g}_{n}$. On $D S(V, \rho)$ we have $\rho(H)=0$ and $\rho(x)=0$. Since

$$
\left[x, x_{i}\right]=\left[x, x_{i}^{\prime}\right]=\left[x, z_{i}\right]=\left[x, z_{i}^{\prime}\right]=0,
$$


the elements $x_{i}, x_{i}^{\prime}, z_{i}, z_{i}^{\prime}$ naturally act on the cohomology $D S(V)=V_{x}$. Since $x$ commutes with $H$, the spaces $\operatorname{Kern}(x)$ and its subspace $\operatorname{Im}(x)$ decompose into $H$-eigenspaces $\operatorname{Kern}(x)(j)$ and $\operatorname{Im}(x)(j)$ for $j \in \mathbb{Z}$. By lemma 5.1 however $\operatorname{Kern}(x)(j)=\operatorname{Im}(x)(j)$, expect for the zero-eigenspace of $H$. Now, although $x, \bar{x}$ commute with $H$, the operators $y \in\left\{x_{i}, x_{i}^{\prime}, z_{i}, z_{i}^{\prime}\right\}$ satisfy $[H, y]= \pm y$ and hence map the zero eigenspace $M=V^{H}$ into the \pm 1 -eigenspace of $H$ on $V$. Since the $j= \pm 1$-eigenspaces do not give a nonzero contribution to the cohomology $D S(V)=V_{x}$, this implies

Lemma 11.1. The natural action of $\rho\left(x_{i}\right), \rho\left(x_{i}^{\prime}\right), \rho\left(z_{i}\right), \rho\left(z_{i}^{\prime}\right)$ and $\rho(x), \rho(H)$ on $D S(V, \rho)$ is trivial.

Notice that $C_{n}$ commutes with all elements in $\mathfrak{g}_{n}$, hence induces a linear map on $D S(V, \rho)$ that commutes with the action of $G_{n-1}$ on $D S(V, \rho)$.

Lemma 11.2. The restriction of the Casimir $C_{n}$ acts on $D S(V, \rho)$ like the Casimir $C_{n-1}$ of $T_{n-1}$ acts on $D S(V, \rho) \in T_{n-1}$.

Proof. By lemma 11.1 the restriction of $C_{n}$ to $D S(V, \rho)$ is the sum of $C_{n-1}$ and the operator $C_{1}=e_{n, n}^{2}-e_{n+1, n+1}^{2}$. Now consider a weight space of $D S(V, \rho)$ with eigenvalue $\bar{\lambda}$. Then $\bar{\lambda}$ is the restriction of an eigenvalues $\lambda$ of the weight decomposition of $(V, \rho)$. Since $D S(V, \rho)$ is represented by elements in $M=V^{H}$, the condition $\lambda(H)=0$ implies $\lambda_{n}=-\lambda_{n+1}$ and hence $\lambda_{n}^{2}-\lambda_{n+1}^{2}=0$. Therefore $C_{1}$ acts trivially on $D S(V, \rho)$.

Remark. As the referee pointed out, there is a more conceptual proof of lemma 11.2. For a module $M \in T_{n}$, the Casimir map is the composition

$$
C_{M}: M \longrightarrow \mathfrak{g l}(n \mid n) \otimes \mathfrak{g l}(n \mid n)^{*} \otimes M \longrightarrow \mathfrak{g l}(n \mid n) \otimes M \longrightarrow M
$$

where the first map is the coevaluation map for the adjoint representation of $\mathfrak{g}$ and the last two are the action maps $\mathfrak{g l}(n \mid n) \otimes M \rightarrow M$. Since $D S$ maps the standard representation to the standard representation, it preserves the adjoint representation as well, and hence preserves the Casimir map in the sense that $D S\left(C_{M}\right)$ is the Casimir on $D S(M)$.

\section{PART 2. The MAIN THEOREM AND ITS PROOF}

In this part we prove the main theorem, stating that $D S(L)=\bigoplus_{i} \Pi^{n_{i}} L\left(\lambda_{i}\right)$ in $T_{n-1}$ for any irreducible representation $L$. We have seen that $D S(L)$ is actually a $\mathbf{Z}$-graded object in $T_{n-1}$; and we calculate the $\mathbf{Z}$-grading for any $L$ in the propositions 23.124 .2 . These statements contain the main theorem as a special case. Their proofs however depend on the main theorem and its proof. We will prove the main theorem first for special irreducible $L$, 
called ground states, and reduce the general question to these by means of translation functors.

\section{The LANGUAGE OF BRUNDAN AND StROPPEL}

By the work of Brundan-Stroppel [BS12a] the block combinatoric and notably the $E x t^{1}$ between irreducible representations can be described in terms of weight and cup diagrams associated to any irreducible $L() \lambda)$.

Weight diagrams. Consider a weight $\lambda=\left(\lambda_{1}, \ldots, \lambda_{n} ; \lambda_{n+1}, \cdots, \lambda_{2 n}\right)$. Then $\lambda_{1} \geq \ldots \geq \lambda_{n}$ and $\lambda_{n+1} \geq \ldots \geq \lambda_{2 n}$ are integers, and every $\lambda \in \mathbb{Z}^{m+n}$ satisfying these inequalities occurs as the highest weight of an irreducible representation $L(\lambda)$. The set of highest weights will be denoted by $X^{+}=$ $X^{+}(n)$. Following [BS12a] to each highest weight $\lambda \in X^{+}(n)$ we associate two subsets of cardinality $n$ of the numberline $\mathbb{Z}$

$$
\begin{aligned}
I_{\times}(\lambda) & =\left\{\lambda_{1}, \lambda_{2}-1, \ldots, \lambda_{n}-n+1\right\} \\
I_{\circ}(\lambda) & =\left\{1-n-\lambda_{n+1}, 2-n-\lambda_{2 n-1}, \ldots,-\lambda_{2 n}\right\} .
\end{aligned}
$$

We now define a labeling of the numberline $\mathbb{Z}$. The integers in $I_{\times}(\lambda) \cap$ $I_{\circ}(\lambda)$ are labeled by $\vee$, the remaining ones in $I_{\times}(\lambda)$ resp. $I_{\circ}(\lambda)$ are labeled by $\times$ respectively $\circ$. All other integers are labeled by $\wedge$. This labeling of the numberline uniquely characterizes the weight vector $\lambda$. If the label $\vee$ occurs $r$ times in the labeling, then $r=$ atyp $(\lambda)$ is called the degree of atypicality of $\lambda$. Notice $0 \leq r \leq n$, and for $r=n$ the weight $\lambda$ is called maximal atypical.

Blocks. A block $\Gamma$ of $X^{+}(n)$ is a connected component of the Ext-quiver of $\mathcal{R}_{n}$. Let $\mathcal{R}_{\Gamma}$ (or by abuse of notation $\Gamma$ ) be the full subcategory of objects of $\mathcal{R}_{n}$ such that all composition factors are in $\Gamma$. This gives a decomposition $\mathcal{R}_{n}=\bigoplus_{\Gamma} \mathcal{R}_{\Gamma}$ of the abelian category. Two irreducible representations $L(\lambda)$ and $L(\mu)$ are in the same block if and only if the weights $\lambda$ and $\mu$ define labelings with the same position of the labels $\times$ and $\circ$. The degree of atypicality is a block invariant, and the blocks $\Lambda$ of atypicality $r$ are in 1-1 correspondence with pairs of disjoint subsets of $\mathbb{Z}$ of cardinality $n-r$ resp. $n-r$. Let $\mathcal{R}_{n}^{i}$ be the full subcategory of $\mathcal{R}_{n}$ defined by the blocks of atypicity $n-i$. In particular $\mathcal{R}_{n}$ has a unique maximally atypical block, and any block of atypicality $i$ in $\mathcal{R}_{n}$ is equivalent to the maximally atypical block in $\mathcal{R}_{i}$.

Cups. To each weight diagram we associate a cup diagram as in [BS11]. Here a cup is a lower semi-circle joining two points in $\mathbb{Z}$. To construct the cup diagram go from left to right throught the weight diagram until one finds a pair of vertices $\vee \wedge$ such that there only $x$ 's, o's or vertices which are already joined by cups between them. Then join $\vee \wedge$ by a cup. This procedure will result in a weight diagram with $r$ cups. For example 


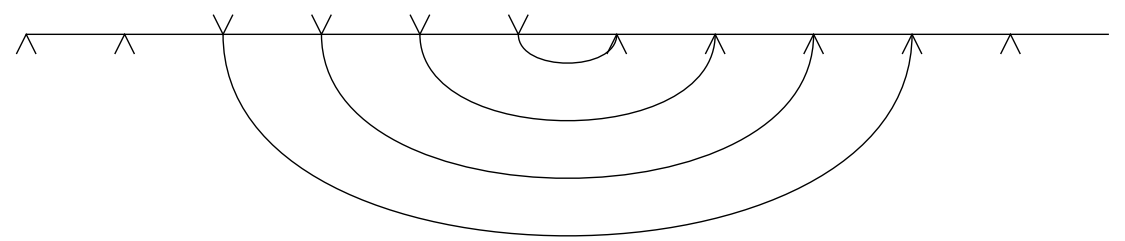

is the labelled cup diagram $(n=4)$ of the trivial representation attached to the weight $\lambda=(0, \ldots, 0 \mid 0, \ldots, 0)$.

Sectors and segments. For the purpose of this paragraph we assume $\lambda \epsilon$ $X^{+}$to be in a maximal atypical block, so that the weight diagram does not have labels $\times$ or $\circ$. Some of the $r$ cups of a cup diagram may be nested. If we remove all inner parts of the nested cups we obtain a cup diagram defined by the (remaining) outer cups. We enumerate these cups from left to right. The starting point of the $j$-th lower cup is denoted $a_{j}$ and its endpoint is denoted $b_{j}$. Then there is a label $\vee$ at the position $a_{j}$ and a label $\wedge$ at position $b_{j}$. The interval $\left[a_{j}, b_{j}\right]$ of $\mathbb{Z}$ will be called the $j$-th sector of the cup diagram. Adjacent sectors, i.e with $b_{j}=a_{j+1}-1$ will be grouped together into segments. The segments again define intervals in the numberline. Let $s_{j}$ be the starting point of the $j$-th segment and $t_{j}$ the endpoint of the $j$-th segment. Between any two segments there is a distance at least $\geq 1$. In the following case the weight diagram has 2 segments and 3 sectors

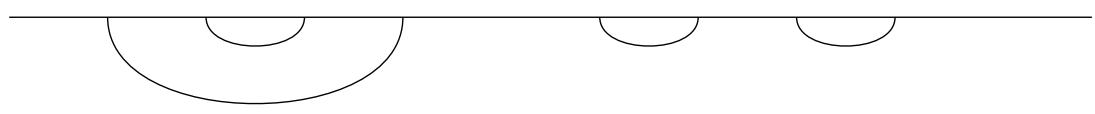

whereas the following weight diagram has 1 segment and 1 sector.

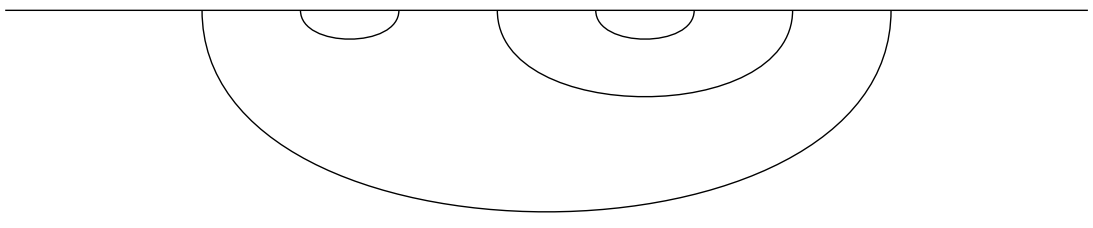

Removing the outer circle would result in a cup diagram with two sectors and one segment. We can also define the notion of a sector or segment for blocks which are not maximally atypical. In this case we say that two sectors are adjacent (and belong to the same segment) if they are only separated by $\times$ or $\circ$ 's. For our purpose the $\times$ and $\circ$ 's will not play a role and we will often implicitly assume that we are in the maximally atypical block.

Important invariants. Note that the segment and sector structure of a weight diagram is completely encoded by the positions of the $\vee$ 's. Hence any finite subset of $\mathbb{Z}$ defines a unique weight diagram in a given block. 
This will lead to the notion of a plot in the next section where we associate to a maximal atypical highest weight the following invariants:

- the type (SD) resp. (NSD),

- the number $k=k(\lambda)$ of sectors of $\lambda$,

- the sectors $S_{\nu}=\left(I_{\nu}, K_{\nu}\right)$ from left to right (for $\nu=1, \ldots, k$ ),

- the ranks $r_{\nu}=r\left(S_{\nu}\right)$, so that $\# I_{\nu}=2 r_{\nu}$,

- the distances $d_{\nu}$ between the sectors (for $\nu=1, \ldots, k-1$ ),

- the total shift factor $d_{0}=\lambda_{n}$

- and the added distances $\delta_{i}=\sum_{\nu=0}^{i-1} d_{\nu}$.

If convenient, $k$ sometimes may also denote the number of segments, but hopefully no confusion will arise from this.

A maximally atypical weight is called basic if $\lambda_{\nu}=-\lambda_{n+\nu}$ holds for $\nu=1, \ldots, n$ such that $[\lambda]:=\left(\lambda_{1}, \ldots, \lambda_{n}\right)$ defines a decreasing sequence $\lambda_{1} \geq$ $\cdots \geq \lambda_{n-1} \geq \lambda_{n}=0$ with the property $n-\nu \geq \lambda_{\nu}$ for all $\nu=1, \ldots, n$. The total number of such basic weights in $X^{+}(n)$ is the Catalan number $C_{n}$. Reflecting the graph of such a sequence $[\lambda]$ at the diagonal, one obtains another basic weight $[\lambda]^{*}$. We will show that a basic weight $\lambda$ is of type (SD) if and only if $[\lambda]^{*}=[\lambda]$ holds. To every maximal atypical highest weight $\lambda$ is attached a unique maximal atypical highest weight $\lambda_{\text {basic }}$

$$
\lambda \mapsto \lambda_{\text {basic }}
$$

having the same invariants as $\lambda$, except that $d_{0}=d_{1}=\cdots=d_{k-1}=0$ holds for $\lambda_{\text {basic }}$. For example, the basic weight attached to the irreducible representation $[5,4,-1]$ in $\mathcal{R}_{3}$ with cup diagram

is the basic representation $[2,1,0]$ with weight diagram

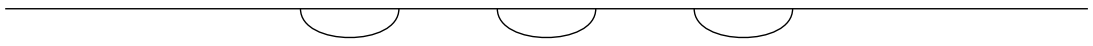

\section{ON SEGMENTS, SECTORS AND PLOTS}

If $\lambda$ is a maximally atypical weight in $\mathcal{R}_{n}$, it is completely encoded by the $n \vee$ 's in its weight diagram. We change the point of view and regard it as a map (a plot) $\lambda: \mathbf{Z} \rightarrow\{\boxplus, \boxminus\}$ where the $\boxminus$ correspond to the $\vee$ 's. If $\lambda$ is not maximal atypical, its weight diagram has crosses and circles. These do not play any role in the combinatorial arguments, and we can still describe $\lambda$ by a plot if we just ignore and remove the crosses and circles from the weight diagram.

A plot $\lambda$ is a map

$$
\lambda: \mathbb{Z} \rightarrow\{\boxplus, \boxminus\}
$$


such that the cardinality $r$ of the fiber $\lambda^{-1}(\boxplus)$ is finite. Then by definition $r=r(\lambda)$ is the degree and $\lambda^{-1}(\boxplus)$ is the support of $\lambda$. As usual an interval $[a, b] \subset \mathbb{Z}$ is the set $\{x \in \mathbb{Z} \mid a \leq x \leq b\}$. Replacing $\boxplus$ by 1 and $\boxminus$ by -1 we may view $\lambda(x)$ as a real valued function extended by $\lambda(x):=\lambda([x])$ to a function on $\mathbb{R}$ for $[x]=\max _{n \in \mathbb{Z}}\{n \leq x\}$.

Segments and sectors. An interval $I=[a, b]$ of even cardinality $2 r$ and a subset $K$ of cardinality of rank $r$ defines a plot $\lambda$ of rank $r$ with support $K$. We call $(I, K)$ a segment, if $f(x)=\int_{a}^{x} \lambda(x) d x$ is nonnegative on $I$. Notice, then $a \in K$ but $b \notin K$.

Factorization. For a given plot $\lambda$ put $a=\min (\operatorname{supp}(\lambda))$ and for the first zero $x_{0}>a$ of the function $f(x)=\int_{a}^{x} \lambda(x) d x$ put $b=x_{0}-1$. This defines an interval $I=[a, b]$ of even length, such that $\left.\lambda\right|_{I}$ (now again viewed as a function on $I \cap \mathbb{Z}$ ) admits the values 1 and -1 equally often. If $\operatorname{supp}(\lambda) \subset I$, then $\lambda$ is called a prime plot. If $\lambda$ is not a prime plot, the plot $\lambda_{1}$ with support $I \cap \operatorname{supp}(\lambda)$ defines a prime plot. It is called the first sector of the plot $\lambda$. Now replace the plot $\lambda$ by the plot, where the support $K_{1}$ of the first sector $I=I_{1}$ is removed from the support $K$ of $\lambda$. Repeating the process above, we obtain a prime plot $\lambda_{2}$ with support $K_{2}$ defining a segment $\left(I_{2}, K_{2}\right)$. This segment is called the second sector of $\lambda$. Obviously $I_{1}$ is an interval in $\mathbb{Z}$ on the right of $I_{1}$, hence in particular they are disjoint. Continuing with this process, one defines finitely many prime plots $\lambda_{1}, \ldots, \lambda_{k}$ attached to a given plot defining disjoint segments $S_{1}=\left(I_{1}, K_{1}\right), \ldots, S_{k}\left(I_{k}, K_{k}\right)$. These segments $S_{\nu}$ are called the sectors of the plot $\lambda$. Let

$$
d_{\nu}=\operatorname{dist}\left(I_{\nu}, I_{\nu+1}\right) \quad, \quad \nu=1, \ldots, k-1
$$

denote the distances between these sectors $S_{\nu}$, i.e. $d_{\nu}=\min \left(S_{\nu+1}\right)-$ $\max \left(S_{\nu}\right)$.

For disjoint segments $\left(I_{1}, K_{1}\right)$ and $\left(I_{1}, K_{2}\right)$ the union $(I, K)=\left(I_{1} \cup\right.$ $\left.I_{2}, K_{1} \cup K_{2}\right)$ again is a segment, provided $I=I_{1} \cup I_{2}$ is an interval in $\mathbb{Z}$.

Grouping together adjacent sectors of $\lambda$ with distances $d_{\nu}=0$ defines the segments of $\lambda$. In other words, the union of the intervals $I_{\nu}$ of the sectors $S_{\nu}$ of the $\lambda_{\nu}$ can be written a disjoint union of intervals $I$ of maximal length. These intervals $I$ define the segments of $\lambda$ as $(I, T \cap \operatorname{supp}(\lambda))$.

We consider formal finite linear combinations $\sum_{i} n_{i} \cdot \lambda_{i}$ of plots with integer coefficients. This defines an abelian group $R=\bigoplus_{r=0}^{\infty} R_{r}$ (graduation by rank $r$ ). We define a commutative ring structure on $R$ so that the product of two plots $\lambda_{1}$ and $\lambda_{2}$ is zero unless the segments of $\lambda_{1}$ and $\lambda_{2}$ are disjoint, in which case the support of the product becomes the union of the supports. A plot $\lambda$ that can not be written in the form $\lambda_{1} \cdot \lambda_{2}$ for plots $\lambda_{i}$ of rank $r_{i}>0$ is called a prime plot. 
Lemma 13.1. Every plot can be written as a product of prime plots uniquely up to permutation of the factors.

Of course this prime factorization of a given plot $\lambda$ is given by the prime factors $\lambda_{\nu}$ attached to the sectors $S_{\nu}, \nu=1, . ., k$ of $\lambda$. Hence for $\lambda=\prod_{i} \lambda_{i}$ with prime plots $\lambda_{i}$, the sectors of $\lambda$ are the segments attached to the prime factors $\lambda_{i}$. The interval $I=[a, \ldots, b]$ attached to a prime plot $\lambda$ is the unique sector or the unique segment of the prime plot $\lambda$. It has cardinality $2 r(\lambda)$, and the support $K$ of $\lambda$ defines a subset of the sector $I$ of cardinality $r$. Recall $a \in K$ but $b \notin K$.

Differentiation. We define a derivation on $R$ called derivative. Indeed the derivative induces an additive map

$$
\partial: R_{n} \rightarrow R_{n-1} .
$$

To differentiate a plot of rank $n>0$, or a segment, we use the formula

$$
\partial\left(\prod_{i} \lambda_{i}\right)=\sum_{i} \partial \lambda_{i} \cdot \prod_{j \neq i} \lambda_{j}
$$

in the ring $R$ to reduce the definition to the case of a prime plot $\lambda$. For prime $\lambda$ let $(I, K)$ be its associated sector. Then $I=[a, b]$. Using $a \in K, b \notin K$, for a sector $(I, K)$ of a prime plot $\lambda$ it is easy to verify by the integral criterion that

$$
\partial(I, K)=(I, K)^{\prime}=\left(I^{\prime}, K^{\prime}\right)
$$

for $I^{\prime}=[a+1, b-1]$ and $K^{\prime}=I \cap K$ again defines the sector of a prime plot $\partial \lambda$ of rank $r\left(\lambda^{\prime}\right)=r(\lambda)-1$. Then for prime plots $\lambda$ of rank $n$ with sector $(I, K)$ we define $\partial \lambda$ in $R$ by

$$
\partial \lambda=\partial(I, K) \quad, \quad I=[a, b] .
$$

Integration. For a segment $(I, K)$ with $I=[a, b]$ put

$$
\int(I, K)=([a-1, b+1], K \cup\{a-1\})
$$

increasing the rank by 1 . Observe, that the integral criterion implies that $([a-1, b+1], K \cup\{a-1\})$ always defines a prime segment. Obviously

$$
\partial \int(I, K)=(I, K) \text {. }
$$

Similarly $\int \partial(I, K)=(I, K)$ for a prime segment $(I, K)$ of rank $>0$.

Lowering sectors. For a sector $S=(I, K)$ with $I=[a, b]$ define

$$
S^{\text {low }}=([a-1, a],\{a-1\}) \cup \partial(S) .
$$

Notice that $S^{l o w}$ is a segment with interval $[a-1, b-1]$. 
Melting sectors. Suppose $\lambda_{1}$ and $\lambda_{2}$ are prime plots. Let $\left(I_{1}, K_{1}\right)$ and $\left(I_{2}, K_{2}\right)$ be their defining sectors. Assume that $(I, K)=\left(I_{1} \cup I_{2}, K_{1} \cup K_{2}\right)$ defines a segment with plot $\lambda$. Hence $I_{1}=[a, i]$ and $I_{2}=[i+1, b]$ for some $i \in \mathbb{Z}$ and $i \notin K_{1}$ and $i+1 \in K_{2}$. Then by the integral criterion

$$
(I, K)^{\text {melt }}=\left(I_{1} \cup I_{2}, K_{1} \cup\{i\} \cup\left(K_{2}-\{i+1\}\right)\right.
$$

defines a prime plot with $I=[a, b]$.

Example. We can represent plots with labelled cup diagrams. A plot of rank $r$ has $r$ cups. For instance the irreducible representation $[3,3,1,1] \in \mathcal{R}_{4}$ has the cup diagram

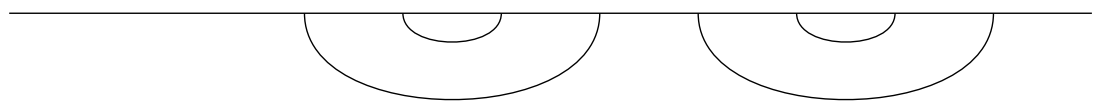

The corresponding plot is defined by its support $\{-2,-1,2,3\}$. Its derivative is the sum of two plots of rank 3 corresponding to the two cup diagrams

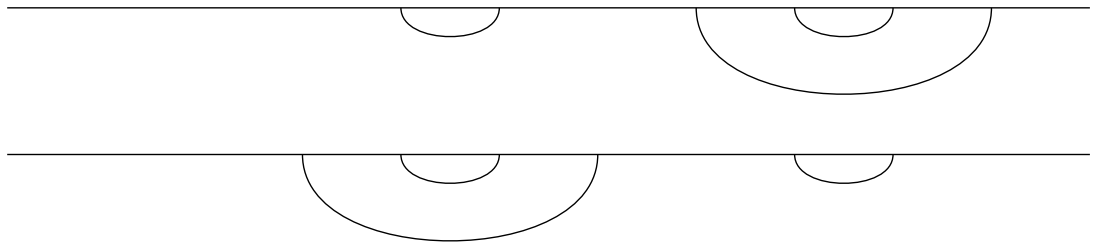

If we integrate the first segment of the plot we get the plot of rank 5 with support $\{-3,-2,-1,2,3\}$ with corresponding cup diagram

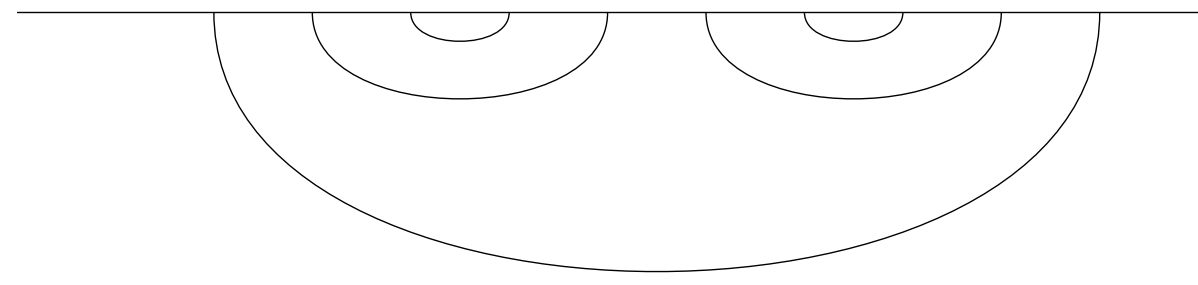

The plot of $[3,3,1,1]$ has two adjacent sectors. Melting these two gives the plot with support $\{-2,-1,1,3\}$ with cup diagram

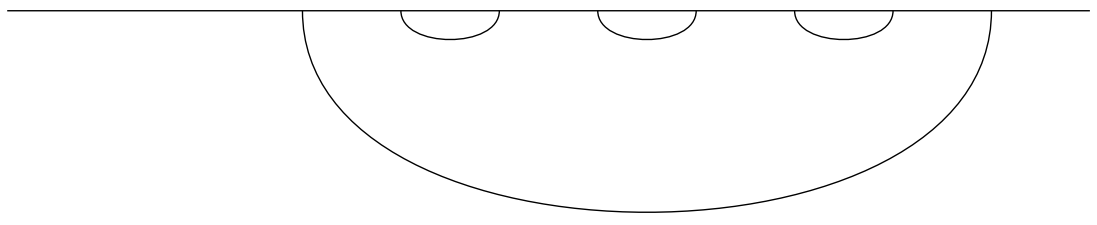


The not maximally atypical case. As with sectors and segments we can define the notion of a plot for representations which are not maximally atypical. We fix the block of the irreducible representations, ie. the positions of the $\times$ 's (say at the vertices $x_{1}, \ldots, x_{r}$ ) and the positions of the $\circ$ 's (say at the vertices $\left.\circ_{1}, \ldots, \circ_{r}\right)$. Once these are fixed we define $\mathbf{Z}_{\times \circ}:=$ $\mathbf{Z} \backslash\left(\left\{x_{1}, \ldots, x_{r}\right\} \cup\left\{\circ_{1}, \ldots, o_{r}\right\}\right)$. Then a plot is a map $\lambda: \mathbf{Z}_{\times \circ} \rightarrow\{\boxplus, \boxminus\}$. The reader can convince himself that all the previous definitions and operations on plots (factorization, derivatives etc) can be adapted easily to this more general setting. However this amounts in practice only to fixing the positions of the $\times$ and $\circ$ 's and then ignoring them. We will associate in section 18 to every weight $\lambda$ of atypicality $i$ a plot $\phi(\lambda)$ of rank $i$ (without $\times$ 's and $\circ$ 's) and work with these instead.

\section{MiXED TENSORS AND GROUND STATES}

We compute $D S(L) \in T_{n-1}$ for special irreducible representations $L$ in a block $\Gamma$, the so-called ground states. The general case for arbitrary $L$ will then be reduced to this case in later sections.

Let $M T$ denote the full subcategory of mixed tensors in $\mathcal{R}_{n}$ whose objects are direct sums of the indecomposable objects in $\mathcal{R}_{n}$ that appear in a decomposition $X_{s t}^{\otimes r} \otimes\left(X_{s t}^{\vee}\right)^{\otimes s}$ for some natural numbers $r, s \geq 0$, where $X_{s t} \in \mathcal{R}_{n}$ denotes the standard representation. By [BS12b] and [CW11] the indecomposable objects in $M T$ are parametrized by $(n \mid n)$-cross bipartitions. Let $R_{n}\left(\lambda^{L}, \lambda^{R}\right)$ denote the indecomposable representation in $\mathcal{R}_{n}$ corresponding to the bipartition $\lambda=\left(\lambda^{L}, \lambda^{R}\right)$ under this parametrization.

To any bipartition $\lambda$ we attach a weight diagram in the sense of [BS11], ie. a labelling of the numberline $\mathbb{Z}$ according to the following dictionary. Put

$$
I_{\wedge}(\lambda):=\left\{\lambda_{1}^{L}, \lambda_{2}^{L}-1, \lambda_{3}^{L}-2, \ldots\right\} \quad \text { and } \quad I_{\vee}(\lambda):=\left\{1-\lambda_{1}^{R}, 2-\lambda_{2}^{R}, \ldots\right\} .
$$

Now label the integer vertices $i$ on the numberline by the symbols $\wedge, \vee, \circ, \times$ according to the rule

$$
\begin{cases}\circ & \text { if } i \notin I_{\wedge} \cup I_{\vee}, \\ \wedge & \text { if } i \in I_{\wedge}, i \notin I_{\vee}, \\ \vee & \text { if } i \in I_{\vee}, i \notin I_{\wedge}, \\ \times & \text { if } i \in I_{\wedge} \cap I_{\vee} .\end{cases}
$$

To any such data one attaches a cup-diagram as in section 12 or [BS11] and we define the following three invariants 


$$
\begin{aligned}
& a(\lambda)=\text { number of crosses } \\
& d(\lambda)=\text { number of cups } \\
& k(\lambda)=a(\lambda)+d(\lambda)
\end{aligned}
$$

A bipartition is $(n \mid n)$-cross if and only if $k(\lambda) \leq n$. By [BS12b] the modules $R\left(\lambda^{L}, \lambda^{R}\right)$ have irreducible socle and cosocle equal to $L\left(\lambda^{\dagger}\right)$ where the highest weight $\lambda^{\dagger}$ can be obtained by a combinatorial algorithm from $\lambda$. Let

$$
\theta: \Lambda \rightarrow X^{+}(n)
$$

denote the resulting map $\lambda \mapsto \lambda^{\dagger}$ between the set of $(n \mid n)$-cross bipartitions $\Lambda$ and the set $X^{+}(n)$ of highest weights of $\mathcal{R}_{n}$.

Theorem 14.1. [Hei14] $R=R(\lambda)$ is an indecomposable module of Loewy length $2 d(\lambda)+1$. It is projective if and only if $k(\lambda)=n$, in which case we have $R=P\left(\lambda^{\dagger}\right)$.

Hence $R$ is irreducible if and only if $d(\lambda)=0$, and then $R=L\left(\lambda^{\dagger}\right)$.

Deligne's interpolating category. For every $t \in k$ there exists the category $\operatorname{Rep}\left(G l_{t}\right)$ defined in [Del07]. This is a $k$-linear pseudoabelian rigid tensor category. By construction it contains an object st of dimension $t$, called the standard representation. Given any $k$-linear pseudoabelian tensor category $C$ with unit object and a tensor functor

$$
F: \operatorname{Rep}\left(G l_{t}\right) \rightarrow C
$$

the functor $F \rightarrow F(s t)$ is an equivalence between the category of $\otimes$-functors of $\operatorname{Rep}\left(G l_{t}\right)$ to $C$ with the category of $t$-dimensional dualisable objects $X \in$ $C$ and their isomorphisms.

In particular, given a dualizable object $X$ of dimension $t$ in a $k$-linear pseudoabelian tensor category, a unique tensor functor $F_{X}: \operatorname{Rep}\left(G l_{t}\right) \rightarrow C$ exists mapping st to $X$. Hence, for our categories $\mathcal{R}_{n}$ and $t=0$, we get a tensor functor $F_{n}: \operatorname{Rep}\left(G l_{0}\right) \rightarrow \mathcal{R}_{n}$ by mapping the standard representation of $\operatorname{Rep}\left(G l_{0}\right)$ to the standard representation of $G l(n \mid n)$ in $\mathcal{R}_{n}$. Every mixed tensor is in the image of this tensor functor ( [CW11]). The indecomposable elements in Deligne's category are parametrized by the set of all bipartitions. The kernel of $F_{n}$ contains those indecomposables labelled by bipartitions that are not $(n \mid n)$-cross. Any $(n \mid n)$-cross bipartion $\lambda$ defines an indecomposable object in the image of $\operatorname{Rep}\left(G l_{0}\right)$. We write $R_{n}(\lambda)$ for $F_{n}(R(\lambda))$. By the universal property of Deligne's category any tensor functor from $\operatorname{Rep}\left(G l_{0}\right)$ to a tensor category $C$ is fixed up to isomorphism by the choice of an image of the standard representation of $\operatorname{Rep}\left(G l_{0}\right)$. 
Lemma 14.2. [Hei14] $D S\left(R_{n}(\lambda)\right)=R_{n-1}(\lambda)$ holds unless $R_{n}(\lambda)$ is projective, in which case $D S\left(R_{n}(\lambda)\right)=0$.

Note that the vanishing of $R_{n}(\lambda)_{x}$ in the projective case is just a special case of lemma 4.2 (i) and (ii).

Proof. An easy computation shows that under the Duflo-Serganova functor the standard representation of $\mathfrak{g}_{n}$ is mapped to the standard representation of $\mathfrak{g}_{n-1}$. Since any indecomposable mixed tensor module is in the image of a tensor functor from Deligne's category $\operatorname{Rep}\left(G l_{0}\right)$ [CW11] the result follows from the commutative diagram

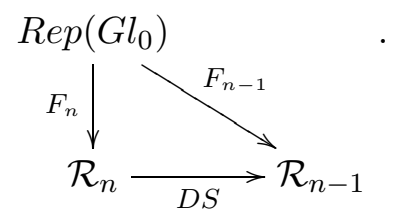

The kernel of $F_{n}$ consists of the $R(\lambda)$ with $k(\lambda)>n$, the kernel of $F_{n-1}$ consists of the $R(\lambda)$ with $k(\lambda) \geq n$ Hence $R(\lambda) \in \operatorname{ker}(D S)$ if and only if $k(\lambda)=n$ which is equivalent to $R(\lambda)$ projective.

Example. As in section 17 put $\mathbb{A}_{S^{i}}:=R\left((i),\left(1^{i}\right)\right) \in \mathcal{R}_{n}$. By lemma 14.2 we have $\left(\mathbb{A}_{S^{i}}\right)_{x}=\mathbb{A}_{S^{i}}$ for all $i \geq 1$.

Corollary 14.3. Every indecomposable projective module of $\mathcal{R}_{n-1}$ is in the image of $D S$.

Proof. The indecomposable projective modules are precisely the modules $D S\left(R\left(\lambda^{L}, \lambda^{R}\right)\right)$ with $k(\lambda)=n-1$. Note that every indecomposable projective module is a mixed tensor [Hei14].

Irreducible mixed tensors. By the results above the map $\theta: \Lambda \rightarrow X^{+}(n)$ is injective if restricted to bipartitions with $d(\lambda)=0$. We denote by $\theta^{-1}$ its partial inverse. A closer inspection [Hei14] of the assignment $\theta: \lambda \mapsto \lambda^{\dagger}$ shows that $\theta$ and $\theta^{-1}$ are given by the following simplified rule: Define

$$
\begin{aligned}
m & =\operatorname{maximal} \text { coordinate of a } \times \text { or } \circ \\
t & =\max (k(\lambda)+1, m+1) \\
s & = \begin{cases}0 & m+1 \leq k(\lambda)+1 \\
m-k(\lambda) & m+1>k(\lambda)+1\end{cases}
\end{aligned}
$$

The weight diagram of $\lambda^{\dagger}$ is obtained from the weight diagram of $\lambda$ by switching all $\vee$ 's to $\wedge$ 's and vice versa at positions $\geq t$ and switching the first $s+n-k(\lambda) \vee$ 's at positions $<t$ to $\vee^{\prime}$ 's and vice versa. The numbers labelled by a $\wedge$ or $\vee$ will be called free positions. Conversely if $L\left(\lambda^{\dagger}\right)$ is some irreducible representation in $M T$, the corresponding bipartition with 
$\theta^{-1}\left(\lambda^{\dagger}\right)=\lambda$ is obtained in the same way: Define $t, m, s$ as above and apply the same switching rules to the weight diagram of $\lambda^{\dagger}$.

Proposition 14.4. Let

$$
L=L\left(\lambda^{\dagger}\right)=L\left(\lambda_{1}, \ldots, \lambda_{n-i}, 0, \ldots, 0 ; 0, \ldots, 0, \lambda_{n+i+1}, \ldots, \lambda_{2 n}\right)
$$

be an irreducible $i$-fold atypical representation. Then $L$ is a mixed tensor $L=R(\lambda)$ for a unique bipartition of defect 0 and $r k=n-i$. Then

$$
D S(L)=R_{n-1}(\lambda)=L\left(\bar{\lambda}^{\dagger}\right),
$$

where $\bar{\lambda}^{\dagger}$ is obtained from $\lambda^{\dagger}$ by removing the two innermost zeros corresponding to $\lambda_{n}^{\dagger}$ and $\lambda_{n+1}^{\dagger}$.

Proof. We apply $\theta^{-1}$ to $\lambda^{\dagger}$. It transforms the weight diagram of $\lambda^{\dagger}$ into some other weight diagram which might not be the weight diagram of a bipartition. However if the resulting weight diagram is the weight diagram of an $(n \mid n)$-cross bipartition of defect 0 , then $\theta(\lambda)=\lambda^{\dagger}$ and $R(\lambda)=L\left(\lambda^{\dagger}\right)$. For $\lambda^{\dagger}$

$$
\begin{aligned}
I_{\times} & =\left\{\lambda_{1}, \lambda_{2}-1, \ldots, \lambda_{n-i}-(n-i)+1,-n+i, \ldots,-n+1\right\} \\
I_{\circ} & =\left\{1-n, 2-n, \ldots, i-n, i+1-n-\lambda_{n+1+i}, \ldots,-\lambda_{2 n}\right\} .
\end{aligned}
$$

Then $I_{\times} \cap I_{\circ}=\{-n+1, \ldots,-n+i\}$ (since the atypicality is $i$ ) and the $n-i$ crosses are at the positions $\lambda_{1}, \lambda_{2}-1, \ldots, \lambda_{n-i}-(n-i)+1$ and the $n-i$ circles at the positions $i+1-n-\lambda_{n+1+i}, \ldots,-\lambda_{2 n}$. Define $m, t, s$ as above. Note that $k(\lambda)=n-i$. We distinguish two cases, either $t=n-i+1$ or $t=m+1$. Assume first $m+1 \leq n-i+1$. Switch all free labels at positions $\geq t$ and the first $n-(n-i)=i$ free labels at positions $<t$. By assumption the $2 n-2 i$ crosses and circles lie at positions $>i-n$ and $<n-i+1$. However there are exactly $2 n-2 i$ such positions. Hence the switches at positions $<t$ turn exactly the $i \vee$ 's at positions $i-n, \ldots, 1-n$ into $\wedge$ 's. In the second case $t=m+1>n-i+1$ switch the first $m+n-2(n-i)$ free labels at positions $<t$. There are exactly $m+n-i$ positions between $m$ and $i-n, m-n+2 i$ switches and $2 n-2 i$ crosses and circles between $i-n$ and $t$. This results in $m-n+i$ free positions between $i-n$ and $t$. The remaining $i$ switches transform the $i \vee$ 's to $\wedge$ 's. Hence in both cases $\theta^{-1}$ transforms the weight diagram of $\lambda^{\dagger}$ into a weight diagram where the rightmost $\wedge$ is at position $i-n$ and the leftmost $\vee$ is at the first free position $>i-n$ and all labels at positions $\geq t$ are given by $\vee$ 's. This is the weigth diagram of a bipartition of defect 0 and rank $n-i$. Indeed the labelling defines the two sets $I_{\wedge}$ and $I_{\vee}$ and this defines two tuples $\lambda^{L}=\left(\lambda_{1}^{L}, \lambda_{2}^{L}, \ldots\right)$ and $\lambda^{R}=\left(\lambda_{1}^{R}, \lambda_{2}^{R}, \ldots\right)$. The positioning of the $\Lambda^{\prime}$ 's implies that $\lambda_{n-i+1}^{L}=0$ and the positioning of the $\vee^{\prime}$ 's implies $\lambda_{t}^{R}=0$. Clearly $\lambda_{1}^{L}=\lambda_{1}>0$ and $\lambda_{1}^{R} \geq 0$. Hence the pair $\lambda:=\left(\lambda^{L}, \lambda^{R}\right)$ is a bipartition (of defect 0 and rank $n-i$ ) and $\theta(\lambda)=\lambda^{\dagger}$. It 
remains to compute the highest weight of $R_{n-1}(\lambda)$. The two sets $I_{\vee}$ and $I_{\wedge}$ and accordingly the weight diagram of $\lambda$ do not depend on $n$. Neither do $t, m, s$ and the switches at positions $\geq t$. To get $\lambda^{\dagger}$ in $\mathcal{R}_{n}$ from $\lambda$ we switch the first $s+n-(n-i)$ free labels $<t$. To get $\lambda^{\dagger}$ in $\mathcal{R}_{n-1}$ from $\lambda$ we switch the first $s+(n-1)-(n-i)$ free labels $<t$. This results in removing the leftmost $\vee$ at position $1-n$.

Ground states. Let $\mathcal{R}_{n}^{i} \subset \mathcal{R}_{n}$ denotes the full subcategory of $i$-atypical objects. Every block in $\mathcal{R}_{n}^{i}$ contains irreducible objects with the property that all $i$ labels $\vee$ are adjacents and to the left of all $n-i$ labels $\times$ and all $n-i$ labels $\circ$. We call such an irreducible object a groundstate of the corresponding block in $\mathcal{R}_{n}^{i}$. Each block in $\mathcal{R}_{n}^{i}$ uniquely defines its groundstate up to a simultaneous shift of the $i$ adjacent labels $\vee$. The weight $\lambda$ of such a groundstate $L(\lambda)$ is of the form

$$
\lambda=\left(\lambda_{1}, \ldots, \lambda_{n-i}, \lambda_{n}, \ldots, \lambda_{n} ;-\lambda_{n}, \ldots,-\lambda_{n}, \lambda_{n+1+i}, \ldots, \lambda_{2 n}\right) .
$$

with $\lambda_{n} \leq \min \left(\lambda_{n-i},-\lambda_{n+1+i}\right)$ (here $\lambda_{n} \mapsto \lambda_{n}-1$ corresponds to the shift of the $i$ adjacent labels $\vee$ ). The coefficients $\lambda_{1}, \ldots, \lambda_{n-i}, \lambda_{n+1+i}, \ldots, \lambda_{2 n}$ determine and are determined by the position of the labels $\times$ and $\circ$ defining the given block in $\mathcal{R}_{n}^{i}$. We define

$$
\bar{\lambda}=\left(\lambda_{1}, \ldots, \lambda_{n-i}, \lambda_{n}, \ldots ; \ldots,-\lambda_{n}, \lambda_{n+1+i}, \ldots, \lambda_{2 n}\right)
$$

by omitting the innermost $\lambda_{n} ;-\lambda_{n}$ pair. Then $L(\bar{\lambda}) \in \mathcal{R}_{n}^{i-1} \subset T_{n-1}$.

Berezin twists. Twisting with $B e r=B e r_{n}$ induces an endofunctor of $\mathcal{R}_{n}^{i}$ and permutes blocks. By a suitable twist one can replace a given block in $\mathcal{R}_{n}^{i}$ such that it contains the groundstate

$$
\lambda^{\prime}=\left(\lambda_{1}-\lambda_{n}, \ldots, \lambda_{n-i}-\lambda_{n}, 0, \ldots, 0 ; 0, \ldots, 0, \lambda_{n+1+i}+\lambda_{n}, \ldots, \lambda_{2 n}+\lambda_{n}\right) .
$$

Proposition 14.5. For a groundstate $L=L(\lambda)$ of a block in $\mathcal{R}_{n}^{i} \subset \mathcal{R}_{n}$ the image $D S(L)$ in $T_{n-1}$ of $L$ under the Duflo-Serganova functor is

$$
D S(L(\lambda))=\Pi^{-\lambda_{n}} L(\bar{\lambda})
$$

for $i>0$ or $D S(L)=0$ for $i=0$.

In particular therefore theorem 16.1 holds for the groundstates $L=L(\lambda)$ of blocks in $\mathcal{R}_{n}^{i} \subset \mathcal{R}_{n}$.

Proof. We can assume $i>0$. Then we can assume $\lambda_{n}=\lambda_{n+1}=0$ by a suitable Berezin twist. Hence

$$
L=R_{n}\left(\lambda^{L}, \lambda^{R}\right)
$$

for an $(n \mid n)$-cross bipartition $\left(\lambda^{L}, \lambda^{R}\right)$ and therefore

$$
D S(L)=R_{n-1}\left(\lambda^{L}, \lambda^{R}\right)
$$


is irreducible of weight $\bar{\lambda}$, i.e. $D S(L(\lambda))=L(\bar{\lambda})$. This proves the claim, since by assumption now $\lambda_{n+1}=0$.

\section{SIGN NORMALIZATIONS}

The main theorem 16.1 asserts in particular that $D S(L)$ is semisimple. In order to show this we define a sign $\varepsilon(L) \in\{ \pm 1\}$ for every irreducible representation $L$ with the property that $\operatorname{Ext}_{\mathcal{R}_{n}}^{1}(L(\lambda), L(\mu))=0$ if $\varepsilon(\lambda)=$ $\varepsilon(\mu)$. More precisely, this sign should satisfy the following conditions:

(1) Let $\mathcal{R}_{n}(\varepsilon)$ denote the full subcategory of all objects whose irreducible constituents $X$ have sign $\varepsilon(X)$. Similarly define the full subcategories $\Gamma(\varepsilon)$ for a block $\Gamma$. Then we require that the categories $\mathcal{R}(+)$ and $\mathcal{R}(-)$ are semisimple categories.

Clearly it is enough to require that the categories $\Gamma(\varepsilon)$ are semisimple. Note that any such sign function is unique on a block $\Gamma$ up to a global sign \pm 1 . Hence if we normalize the sign by $\varepsilon(L)=+1$ where $L$ is an irreducible representation in a block $\Gamma$, the sign is uniquely determined on $\Gamma$. Our second condition is:

(2) $\varepsilon(L(\lambda))=1$ if $L(\lambda)$ is an irreducible mixed tensor (see section 14) and $\varepsilon(\mathbf{1})=1$.

If $L(\lambda)$ is maximal atypical, we put

$$
\varepsilon(L(\lambda))=(-1)^{p(\lambda)}
$$

for the parity $p(\lambda)=\sum_{i=1}^{n} \lambda_{n+i}$. In the maximal atypical case we have $\operatorname{Ext}_{\mathcal{R}_{n}}^{1}(L(\lambda), L(\mu))=0$ if $p(\lambda) \equiv p(\mu) \bmod 2$ by [Wei10]. Hence the categories $\Gamma_{n}( \pm)$ are semisimple. This determines the sign $\varepsilon$ up to a global \pm 1 on each block $\Gamma$ of atypicality $i$. Indeed by [Ser06] any block of atypicality $i$ is equivalent to the maximal atypical block $\Gamma_{i}$ of $\mathcal{R}_{i}$. We fix once and for all a particular equivalence denote it by $\tilde{\phi}_{n}^{i}$. We describe the effect of $\tilde{\phi}_{n}^{i}$ on an irreducible module $L(\lambda)$ of atypicality $i$ [Ser06] [GS10]. For an $i$-fold atypical weight in $X^{+}(n)$ its weight diagram has $n-i$ vertices labelled with $\times$ and $n-i$ vertices labelled with $\circ$. Let $j$ be the leftmost vertex labelled either by $x$ or $\circ$. By removing this vertex and shifting all vertices at the positions $>j$ one position to the left, recursively we remove all vertices labelled by $\times$ or $\circ$ from the given weight diagram. The remaining finite subset $K$ of labels $\vee$ has cardinality $i$ and the weight diagram so obtained defines a unique irreducible maximally atypical module in $\mathcal{R}_{i}$. Under $\tilde{\phi}_{n}^{i}$ the irreducible representation $L(\lambda)$ maps to the irreducible $G l(i \mid i)$-representation described by the removal of crosses and circles above. We denote the weight of this irreducible representation by $\phi_{n}^{i}(\lambda)$. We make the preliminary definition $\varepsilon(L(\lambda))=(-1)^{p\left(\phi_{n}^{i}(L(\lambda))\right)}$ for a weight $\lambda$ of atypicality $i$. We claim 
that this sign satisfies condition 1 . Indeed in the maximal atypical case we have $\operatorname{Ext}_{\mathcal{R}_{i}}^{1}(L(\lambda), L(\mu))=0$ if $p(\lambda) \equiv p(\mu) \bmod 2$ by [Wei10]. Hence the categories $\Gamma_{i}( \pm)$ are semisimple. The equivalence $\left(\tilde{\phi}_{n}^{i}\right)^{-1}$ between the two abelian categories $\Gamma_{i}$ and $\Gamma$ is exact and sends the semisimple category $\Gamma_{i}( \pm) \subset \mathcal{R}_{i}$ to the category $\Gamma( \pm)$ by the definition of the sign $\varepsilon$; and $\Gamma( \pm)$ is semisimple.

This preliminary definition however doesn't satisfy condition 2 . If $L=$ $L\left(\lambda_{1}, \ldots, \lambda_{n-i}, 0, \ldots, 0 ; 0, \ldots, 0, \lambda_{n+i+1}, \ldots, \lambda_{2 n}\right)$ is the $i$-atypical mixed tensor of section 14, its weight diagram has $n-i$ circles at the vertices $\lambda_{1}, \lambda_{2}-1, \ldots, \lambda_{n-i}-(n-i)+1$ and $n-i$ circles at the vertices $i+1-n-$ $\lambda_{n+1+i}, \ldots,-\lambda_{2 n}$. The $i \vee$ 's are to the left of the crosses and circles. Applying $\tilde{\phi}_{n}^{i}$ removes the crosses and circles but leaves the $\vee$ 's unchanged at the vertices $-n+1, \ldots,-n+i$. The irreducible representation of $G l(i \mid i)$ so obtained is $B e r^{-n+i}$ with $p([-n+i, \ldots,-n+i])=i(-n+i)$. Hence the preliminary sign $\varepsilon(L(\lambda))$ of a mixed tensor of atypicality $i$ is $\varepsilon(L(\lambda))=(-1)^{i(-n+i)}$. In order to satisfy condition 2 ) we have to normalize the sign by the additional factor $(-1)^{i(-n+i)}$ for an $i$-atypical weight, and we define

$$
\varepsilon(\lambda)=(-1)^{i(-n+i)}(-1)^{p\left(\tilde{\phi}_{n}^{i}(L(\lambda))\right)}
$$

where $p$ is the parity in the maximal atypical block of $G l(i \mid i)$. This sign satisfies condition 1) and 2) by construction, and it is the unique sign with these properties. Note that our definition implies that the sign of a typical weight in $\mathcal{R}_{n}$ is always positive.

The additional sign factor can be understood as follows. The unique irreducible mixed tensor should play an analogous rule to the trivial representation 1 in $\mathcal{R}_{i}$. W can modify the block equivalences $\tilde{\phi}_{n}^{i}$ as follows: Since the mixed tensor $L(\lambda)$ maps to $B e r^{-n+i}$ we twist with the inverse and define the normalized equivalence

$$
\phi_{n}^{i}(L)=\operatorname{Ber}^{n-i} \otimes \tilde{\phi}_{n}^{i}(L) .
$$

Then we obtain $(-1)^{p\left(\phi_{n}^{i}(L(\lambda))\right)}=(-1)^{-i(-n+i)}(-1)^{p\left(\tilde{\phi}_{n}^{i}(L(\lambda))\right)}$. Hence

$$
\varepsilon(\lambda)=(-1)^{p\left(\phi_{n}^{i}(L(\lambda))\right)} .
$$

Corollary 15.1. The categories $\mathcal{R}_{n}(\varepsilon)$ are semisimple categories.

The sign $\varepsilon$ will automatically have the following important property: The translation functors of section $18 \mathbb{A}=F_{i} L\left(\lambda_{\times_{0}}\right)$ have Loewy structure $(L, A, L)$ with $L \in \mathcal{R}_{n}( \pm \varepsilon)$ and $A \in \mathcal{R}_{n}(\mp \varepsilon)$. This is required in our axioms in section 19 . This property follows immediately from the maximal atypical case [Wei10] due to the description of the composition factors of $F^{i} L\left(\lambda_{\times \circ}\right)$ given in section 18. 
The significance of the sign function is the following: $D S(L)$ is in general a representation in $T_{n-1}$, not $\mathcal{R}_{n-1}$. The sign factor regulates whether an irreducible summand of $D S(L)$ is in $\mathcal{R}_{n-1}$ or $\Pi \mathcal{R}_{n-1}$, see theorem 16.1.

In the proof of the main theorem we use the language of plots of section 13 for uniform bookkeeping. Each maximally atypical irreducible representation $L$ defines a plot: The segments of the cup diagram of $L$ define the segments of the plot, and the $V$ in the weight diagram give the support of the plot. If $L$ is not maximally atypical, we associate to it a plot via the map $\phi$ of section 18. For each plot we defined its derivative $\partial \lambda$ in section 13. If $\lambda$ is a prime plot given by the sector $(I, K), I=[a, b]$, then $\partial(I, K)=(I, K)^{\prime}=\left(I^{\prime}, K^{\prime}\right)$ for $I^{\prime}=[a+1, b-1]$ and $K^{\prime}=I^{\prime} \cap K=[a, b]$ defines the derivative. We normalize the derivative of section 13 and put

$$
\lambda^{\prime}=(-1)^{a+n-1} \partial(I, K)
$$

where $I=[a, b]$ (i.e. the leftmost $\vee$ is at $a$ ). The reason for this is as follows. The sign has to be normalized in such a way that for objects $X=L(\lambda)$ in the stable range of the given block we get $d(X)=X^{\prime}$ for the map $d$ of section 19 . Assume first that we are in the maximally atypical $\mathcal{R}_{n}$-case and consider a weight with associated prime plot $\lambda$. The parity of the weight $\lambda$ is $p(\lambda)=\sum_{i=1}^{n} \lambda_{n+i}$. Applying $D S$ removes the $\vee$ in the outer cup. The parity of the resulting weight in $T_{n-1}$ is given by $p\left(\lambda^{\prime}\right)=\sum_{i=1}^{n-1} \lambda_{n+i}$, hence $p(\lambda)-p\left(\lambda^{\prime}\right)=\lambda_{n}$ and we get a shift by $n_{i} \equiv(-1)^{\lambda_{n}}$ according to theorem 16.1. The leftmost $\vee$ is at the vertex $a=\lambda_{n}-n+1$, hence $(-1)^{a+n-1}=$ $(-1)^{\lambda_{n}}$ and the two shifts agree.

Let us now assume $a t(L(\lambda))=k<n$ and that the weight defines a prime plot of rank $k$. Here we have to use the normalized plot associated to the weight $\lambda$ by the map $\phi$ from section 18 in which case the two shifts agree again. We may pass to the maximally atypical case due to the lemmas 18.8 , $18.9,18.10$ which allow us to shift all the circles and crosses sufficiently far to the right.

\section{THE MAIN THEOREM}

In the main theorem we calculate $D S(L) \in T_{n-1}$ for any irreducible $L$. We refine this in section 23, 24 and compute the Z-grading of $D S(L)$.

Theorem 16.1. Suppose $L(\lambda) \in \mathcal{R}_{n}$ is an irreducible atypical representation, so that $\lambda$ corresponds to a cup diagram

$$
\bigcup_{j=1}^{r}\left[a_{j}, b_{j}\right]
$$


with $r$ sectors $\left[a_{j}, b_{j}\right]$ for $j=1, \ldots, r$. Then

$$
D S(L(\lambda)) \cong \bigoplus_{i=1}^{r} \Pi^{n_{i}} L\left(\lambda_{i}\right)
$$

is the direct sum of irreducible atypical representations $L\left(\lambda_{i}\right)$ in $\mathcal{R}_{n-1}$ with shift $n_{i} \equiv \varepsilon(\lambda)-\varepsilon\left(\lambda_{i}\right)$ modulo 2 . The representation $L\left(\lambda_{i}\right)$ is uniquely defined by the property that its cup diagram is

$$
\left[a_{i}+1, b_{i}-1\right] \cup \bigcup_{j=1, j \neq i}^{r}\left[a_{j}, b_{j}\right],
$$

the union of the sectors $\left[a_{j}, b_{j}\right]$ for $1 \leq j \neq i \leq r$ and (the sectors occuring in) the segment $\left[a_{i}+1, b_{i}-1\right]$.

Consequence. In particular this implies that for irreducible representation $(V, \rho)$ the $G_{n-1}$-module $H^{+}(V) \oplus H^{-}(V)$ is semisimple in $\mathcal{R}_{n-1}$ and multiplicity free. Furthermore the sign of the constituents in $H^{ \pm}(V)$ is $\pm \operatorname{sign}(V)$.

If we use the language of plots, the main theorem says that the irreducible summands of $D S(L)$ are given by the derivatives of the sectors of the plot associated to $\lambda$.

Example. The maximally atypical weight $[3,0,0]$ has cup diagram

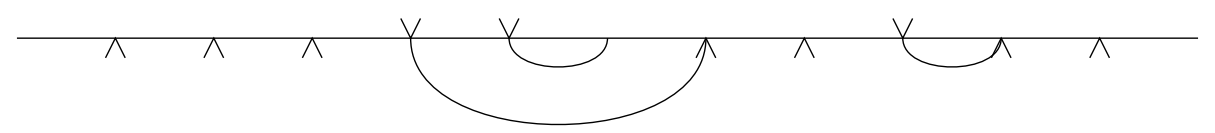

It splits into the two irreducible representations $[3,0]$ and $\Pi[-1,-1]$ in $\mathcal{R}_{2} \oplus \Pi \mathcal{R}_{2}$. We will later compute its cohomology in proposition 23.1 and obtain $H^{\bullet}([3,0,0])=S^{2}\langle 0\rangle \oplus \operatorname{Ber}^{-1}\langle-1\rangle$.

Example. Denote by $S^{i}$ the irreducible representation $[i, 0, \ldots, 0]$. Consider a nontrivial extension $0 \rightarrow S^{2} \rightarrow E \rightarrow \operatorname{Ber}\left(S^{2}\right)^{\vee} \rightarrow 0$ in $\mathcal{R}_{3}$ (such extensions exist). Then $\operatorname{sdim}(E)=0$ and $E$ is indecomposable, hence negligible. The derivative of $S^{2}=[2,0,0]$ (in the sense of plots) is $\left(S^{2}\right)^{\prime}=[2,0]+$ $B e r^{-1}$ and the derivative of $\operatorname{Ber}\left(S^{2}\right)^{\vee}=[2,2,1]$ is $[2,2,1]^{\prime}=(\operatorname{Ber}[1,1,0])^{\prime}=$ $-\operatorname{Ber}\left([1,1,0]^{\prime}\right)=-[2,2]-[2,0]$. From $[2,2]=\operatorname{Ber}^{2}$ then $H^{+}(E)=\operatorname{Ber}^{-1} \oplus$ ? and $H^{-}(E)=B e r^{2} \oplus$ ? where ? is either $[2,0]$ or zero. Hence $E$ is negligible in $\mathcal{R}_{3}$, but $D^{2}(E)=D(D(E)) \neq 0$. In particular, $D\left(\mathcal{N}_{3}\right)$ is not contained in $\mathcal{N}_{2}$.

Block equivalences. Applying $D S$ is compatible with taking the block equivalences $\phi_{n}^{i}$ and $\tilde{\phi}_{n}^{i}$ in the sense $D S\left(\phi_{n}^{i}(L)\right)=\phi_{n}^{i}(D S(L))$ for irreducible $L$ by the main theorem. This can be extended to arbitrary modules $M$ in a block. By [Ser06] and [Kuj11] the block equivalence $\tilde{\phi}_{n}^{i}$ between 
an $i$-atypical block $\Gamma$ and the unique maximal atypical block of $G l(i \mid i)$ is obtained as a series of translation functors, a restriction and the projection onto a weight space. Call a weight $\lambda$ in $\Gamma$ stable if all the $\vee$ 's are to the left of all crosses and circles. By [Ser06] we can apply a suitable sequence of translation functors to any indecomposable module in $\Gamma$ until all its composition factors are stable. We recall now the definition of $\tilde{\phi}_{n}^{i}$ as in [Kuj11] on an indecomposable module $M$. Embed $\mathfrak{g l}(k \mid k)$ as an inner block matrix in $\mathfrak{g l}(n \mid n)$. Let $\mathfrak{l}=\mathfrak{g l}(k \mid k)+\mathfrak{h}$ where $\mathfrak{h}$ are the diagonal matrices. Then choose $\mathfrak{h}^{\prime} \subset \mathfrak{h}$ such that $\mathfrak{h}^{\prime}$ is a central subalgebra of $\mathfrak{l}$ and $\mathfrak{l}=\mathfrak{g l}(k \mid k) \oplus \mathfrak{h}^{\prime}$. We denote the restriction of a weight $\lambda$ to $\mathfrak{h}^{\prime}$ by $\lambda^{\prime}$. Now move $M$ by a suitable sequence of translation functors until its composition factors are stable. The block $\Gamma$ is the full subcategory of modules admitting some central character $\chi_{\mu}$. Now define $\operatorname{Res}_{\mu^{\prime}}(M)=\left\{m \in M \mid h^{\prime} m=\mu^{\prime}\left(h^{\prime}\right) m\right.$ for all $\left.h^{\prime} \in \mathfrak{h}^{\prime}\right\}$. Then, on a module $M$ with stable composition factors, the functor $\tilde{\phi}_{n}^{i}(M)$ is given by $\operatorname{Res}_{\mu^{\prime}}(M)$. Alternatively we could first restrict to $\mathfrak{l}$ and then project on the $\mu^{\prime}$-eigenspace. By [Ser10], cor 4.4, and the main theorem, $D S$ induces a bijection between the blocks in $T_{n}$ and $T_{n-1}$, and for any $M$ in $T_{n} D S\left(F_{i}(M)\right)=F_{i}(D S(M))$. Since our fixed $x$, which we choose in the definition of $D S$, is contained in the embedded $\mathfrak{g l}(k \mid k)$, the operation of $\rho(x)$ on $\operatorname{Res}(M)$ or on its $\lambda^{\prime}$-eigenspace is the same as of $\rho(x)$ on $M$. Hence $D S$ is clearly compatible with restriction, but it also doesn't matter whether we first apply $D S$ and project onto the $\lambda^{\prime}$-eigenspace or first project to the $\lambda^{\prime}$-eigenspace and then apply $D S$ since $\rho(x)$ commutes with $\mathfrak{h}^{\prime}$. Hence $D S\left(\tilde{\phi}_{n}^{i}(M)\right)=\tilde{\phi}_{n}^{i}(D S(M))$ holds for any $M$, and the analogous statement for $\phi_{n}^{i}$ follows immediately. To summarize: If $\Gamma^{\prime}$ denotes the unique block obtained from the $i$-atypical $\Gamma$ via $D S$, we obtain a commutative diagram

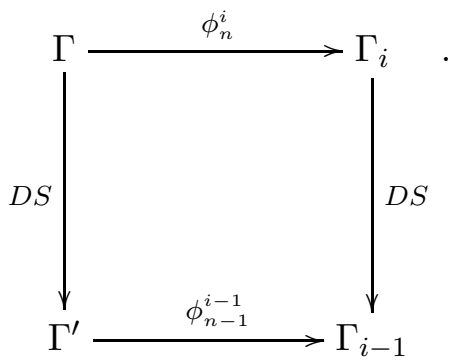

The main theorem has a number of useful consequences:

Cohomology. The main theorem permits us to compute the cohomology $H^{i}(L)$ of irreducible modules $L$ in section 23 and 24. Although the calculation of the $\mathbf{Z}$-grading of $D S(L)$ is much stronger than the $\mathbf{Z}_{2}$ version of 
theorem 16.1, it should be noted that the proof is based on the main theorem and a careful bookkeeping of the moves in section 20 .

Spectral sequences. The main theorem also shows the degeneration of the spectral sequences from section 8 and shows

$$
D S_{n, n_{2}}(L) \simeq D S_{n_{1}, n_{2}}\left(D S_{n, n_{1}}(L)\right) .
$$

The degeneration can be extended in a similar way to the not maximally atypical case, see below.

Tensor products. The main theorem allows us to reduce some questions about tensor products of irreducible representations to lower rank. Since $D S$ is a tensor functor we have $D S(L(\lambda) \otimes L(\mu))=D S(L(\lambda)) \otimes D S(L(\mu))=$ $\bigoplus_{i, j}\left(\Pi^{n_{i}} L\left(\lambda_{i}\right)\right) \otimes\left(\Pi^{n_{j}} L\left(\mu_{j}\right)\right)$. If we inductively understood the tensor product in $T_{n-1}$, we would obtain estimates about the number of indecomposable summands and composition factors in this way. We use this method to calculate the tensor product of two maximal atypical representations of $G l(2 \mid 2)$ in [HW15a], see also [Hei15].

Negligible modules and branching laws. The functor $D S$ does not preserve negligible modules as the example above shows. However when we restrict $D S$ to the full subcategory $\mathcal{R} \mathcal{I}_{n}$ of modules which arise in iterated tensor products of irreducible representations, $D S$ induces a functor $D S: \mathcal{R} \mathcal{I}_{n} / \mathcal{N} \rightarrow \mathcal{R} \mathcal{I}_{n-1} / \mathcal{N}$. We show in [HW15b] [Hei15] that $\mathcal{R} \mathcal{I}_{n} / \mathcal{N}$ is equivalent as a tensor category to the representation category of a proreductive group $H_{n}$. We also show that there is an embedding $H_{n-1} \rightarrow H_{n}$, and $D S$ can be identified with the restriction functor with respect to this embedding. In other words $D S$ gives us the branching laws for the restriction of the image of $L(\lambda)$ in $\operatorname{Rep}\left(H_{n}\right)$ to the subgroup $H_{n-1}$.

Superdimensions and modified superdimensions. The main theorem can be used to reprove parts of the generalized Kac-Wakimoto conjecture on modified superdimensions [Ser10]. In fact we derive a closed formula for the modified superdimension. We sketch this and prove the analog of proposition 8.3.

A superdimension formula. Assume $L$ maximally atypical. If $\operatorname{sdim}(L)>$ 0 ,

$$
D S(L(\lambda)) \cong \bigoplus_{i=1}^{r} \Pi^{n_{i}}\left(L\left(\lambda_{i}\right)\right)
$$

splits into a direct sum of irreducible modules of positive superdimension. Indeed the parity shift $\Pi^{n_{i}}$ occurs if and only if $p(\lambda) \not \equiv p\left(\lambda_{i}\right) \bmod 2$. Hence $D S^{n-1}(L)$ splits into a direct sum of irreducible representations of superdimension 1. Applying $D S n$-times gives a functor $D S^{n}: \mathcal{R}_{n} \rightarrow s v e c$, hence $D S^{n}(L) \simeq m k \oplus m^{\prime} \Pi k$ for positive integers $m, m^{\prime}$, hence $m=0$ if and 
only if $\operatorname{sdim}(L)<0$ and $m^{\prime}=0$ if and only $\operatorname{sdim}(L)>0$. By [Wei10] the superdimension of a maximal atypical irreducible representation in $\mathcal{R}_{n}$ is given by

$$
\operatorname{sim}(L(\lambda))=(-1)^{p(\lambda)} m(\lambda)
$$

for a positive integer $m(\lambda)$ (see below for the definition). In particular

$$
m(\lambda)= \begin{cases}m & p(\lambda) \equiv 0 \bmod 2 \\ m^{\prime} & p(\lambda) \equiv 1 \bmod 2 .\end{cases}
$$

By proposition 8.3 this also holds for $D S_{n, 0}: \mathcal{R}_{n} \rightarrow$ svec: If $D S_{n, 0}(L) \simeq$ $m k \oplus m^{\prime} \Pi k$, we get that either $m$ or $m^{\prime}$ is zero.

The positive integer $m(\lambda)$ for a maximally atypical weight can be computed as follows. We refer to [Wei10], but it would be an easy exercise to deduce this from the main theorem. We let $\underline{\lambda}$ be the associated oriented cup diagram to the weight $\lambda$ as defined in section 12. To each such cup diagram we can associate a forest $\mathcal{F}(\lambda)$ with $n$ nodes, i.e. a disjoint union of rooted trees as in [Wei10]. Each sector of the cup diagram corresponds to one rooted planar tree. We read the nesting structure of the sector from the bottom to the top such that the outer cup corresponds to the root of the tree. If the following is a sector of a cup diagram

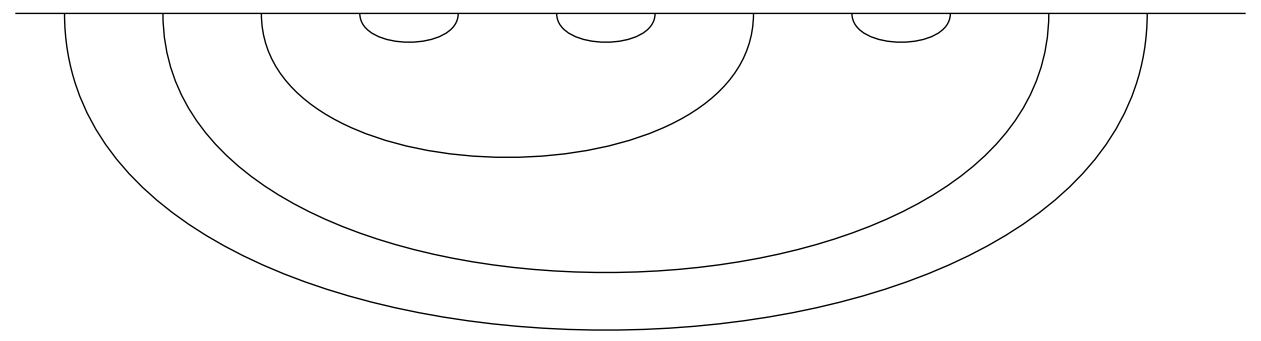

then the associated planar rooted tree is

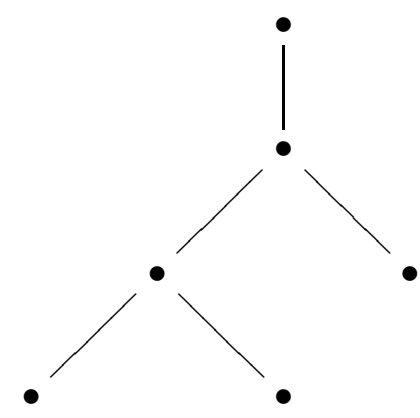

If $\mathcal{F}$ is a forest let $|\mathcal{F}|$ the number of its nodes. We define the forest factorial $\mathcal{F}$ ! as the the product $\prod_{x \in \mathcal{F}}\left|\mathcal{F}_{x}\right|$ where $\mathcal{F}_{x}$ for a node $x \in \mathcal{F}$ denotes 
the subtree of $\mathcal{F}$ rooted at the node $x$. Then the multiplicity is given by

$$
m(\lambda)=\frac{|\mathcal{F}(\lambda)| !}{\mathcal{F}(\lambda) !} .
$$

For example $m(\lambda)$ for irreducible module in $\mathcal{R}_{4}$ with cup diagram

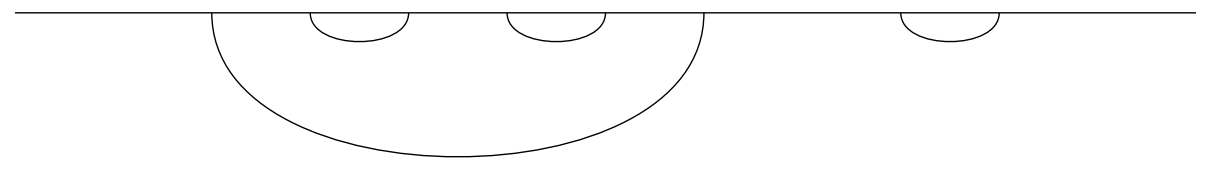

is computed as follows: The associated planar forest is

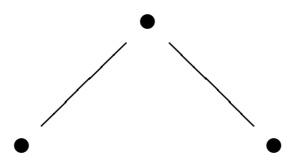

Hence

$$
\operatorname{sdim}(L(\lambda))=\frac{24}{3 \cdot 1 \cdot 1 \cdot 1}=8
$$

Modified Superdimensions If at $(L(\lambda))<n, \operatorname{sdim}(L)=0$. However one can define a modified superdimension for $L$ as follows. We recall some definitions and results from [Kuj11], [GKPM11] and [Ser10]. Denote by $c_{V, W}: V \otimes W \rightarrow W \otimes V$ the usual flip $v \otimes w \mapsto(-1)^{p(v) p(w)} w \otimes v$. Put $e v_{V}^{\prime}=e v_{V} \circ c_{V, V^{\vee}}$ and $\operatorname{coev}_{V}^{\prime}=c_{V, V^{\vee}} \circ \operatorname{coev}_{V}$ for the usual evaluation and coevaluation map in the tensor categories $\mathcal{R}_{n}$ and $T_{n}$. For any pair of objects $V, W$ and an endomorphism $f: V \otimes W \rightarrow V \otimes W$ we define

$$
\begin{aligned}
& \operatorname{tr}_{L}(f)=\left(e v_{V} \otimes i d_{W}\right) \circ\left(i d_{V^{\vee}} \otimes f\right) \circ\left(\operatorname{coev}_{V}^{\prime} \circ i d_{w}\right) \in \operatorname{End}_{T}(W) \\
& \operatorname{tr}_{R}(f)=\left(i d_{V} \otimes e v_{W}^{\prime}\right) \circ\left(f \otimes i d_{W}\right) \circ\left(i d_{V} \otimes \operatorname{coev}_{W}\right) \in \operatorname{End}_{T}(V)
\end{aligned}
$$

For an object $J \in \mathcal{R}_{n}$ let $I_{J}$ be the tensor ideal generated by $J$. A trace on $I_{J}$ is by definition a family of linear functions

$$
t=\left\{t_{V}: \operatorname{End}_{\mathcal{R}_{n}}(V) \rightarrow k\right\}
$$

where $V$ runs over all objects of $I_{J}$ such that following two conditions hold.

(1) If $U \in I_{J}$ and $W$ is an object of $\mathcal{R}_{n}$, then for any $f \in \operatorname{End}_{\mathcal{R}_{n}}(U \otimes W)$ we have

$$
t_{U \otimes W}(f)=t_{U}\left(t_{R}(f)\right) .
$$

(2) If $U, V \in I$ then for any morphisms $f: V \rightarrow U$ and $g: U \rightarrow V$ in $\mathcal{R}_{n}$ we have

$$
t_{V}(g \circ f)=t_{U}(f \circ g) .
$$


By Kujawa [Kuj11], thm 2.3.1, the trace on the ideal $I_{L}, L$ irreducible, is unique up to multiplication by an element of $k$. Given a trace on $I_{J}$, $\left\{t_{V}\right\}_{V \in I_{J}}, J \in \mathcal{R}_{n}$, define the modified dimension function on objects of $I_{J}$ as the modified trace of the identity morphism:

$$
d_{J}(V)=t_{V}\left(i d_{V}\right) .
$$

We reprove the essential part of the generalized Kac-Wakimoto conjecture: We prove that there exists a nontrivial trace on the ideal of any $i$ atypical irreducible $L$, and we deduce a formula for the resulting modified superdimension.

Tensor ideals. By [Ser10] any two irreducible object of atypicality $k$ generate the same tensor ideal. Therefore write $I_{i}$ for the tensor ideal generated by any irreducible object of atypicality $i$. Clearly $I_{0}=\operatorname{Proj}$ and $I_{n}=T_{n}$ since it contains the identity. This gives the following filtration

$$
\operatorname{Proj}=I_{0} \subsetneq I_{1} \subsetneq \ldots I_{n-1} \subsetneq I_{n}=T_{n}
$$

with strict inclusions by [Ser10] and [Kuj11]. We use this in the following. However it is not necessary for the results about the modified superdimension. We could simply consider consider the ideal $\langle L\rangle$ generated by an $i$-atypical irreducible representation instead the ideal $I_{i}$.

The projective case. Denote by $\Delta_{0}^{+}$the positive even roots and by $\Delta_{1}^{+}$ the positive odd roots for our choice of Borel algebra. The half sums of the positive even roots is denoted $\rho_{0}$, the half-sum of the positive odd roots by $\rho_{1}$ and we put $\rho=\rho_{0}-\rho_{1}$. We define a bilinear form $($,$) on \mathfrak{h}^{*}$ as follows: We put $\left(\epsilon_{i}, \epsilon_{j}\right)=\delta_{i j}$ for $i, j \leq m,\left(\epsilon_{i}, \epsilon_{j}\right)=-\delta_{i j}$ for $i, j \geq m+1$ and $\left(\epsilon_{i}, \epsilon_{j}\right)=0$ for $i \leq m$ and $j>m$. Define for any typical module the following function

$$
d(L(\lambda))=\prod_{\alpha \in \Delta_{0}^{+}} \frac{(\lambda+\rho, \alpha)}{\rho, \alpha} / \prod_{\alpha \in \Delta_{1}^{+}}(\lambda+\rho, \alpha) .
$$

Then $d(L(\lambda)) \neq 0$ for every typical $L(\lambda)$. By [GKPM11], 6.2.2 for typical $L$

$$
d_{J}(L)=\frac{d(L)}{d(J)}
$$

Since the ideal $I_{0}$ is independent of the choice of a particular $J$ and any ambidextrous trace is unique up to a scalar, we normalize and define the modified normalized superdimension on $I_{0}$ to be

$$
\operatorname{sdim}_{0}(L(\lambda)):=d(L(\lambda)) .
$$

A formula for the modified superdimension. Applying $D S$ iteratively $i$ times to a module of atypicality $i$ we obtain the functor

$$
D S^{i}:=D S \circ \ldots \circ D S: \mathcal{R}_{n} \rightarrow T_{n-i}
$$


which sends $M$ with atyp $(M)=i$ to a direct sum of typical modules.

We show that there exists a nontrivial trace on $I_{i}$ similar to [Kuj11], but without invoking Serganovas results. Denote by $t^{P}$ the normalized (such that we get $\operatorname{sdim}_{0}$ from above) trace on $I_{0}=\operatorname{Proj}$. Now we define for $M \in I_{i}$

$$
t_{M}(f):=t_{D S^{i}(M)}^{p} f_{D S^{i}(M)}: \operatorname{End}_{\mathcal{R}_{n}}(M) \rightarrow k
$$

where $f_{D S^{i}(M)}$ is the image of $f$ under the functor $D S^{i}$. We claim that this defines a nontrivial trace on $I_{i}$ : Let $M=L$ be irreducible and put

$$
t_{L}\left(i d_{L}\right):=t_{D S^{i}(L)}^{p}\left(i d_{D S^{i}(L)}\right) .
$$

Now we compute $D S^{i}(L)$. By the main theorem the irreducible summands in $D S(L)$ are obtained by removing one of the outer cups of each sector. Applying $D S i$-times gives then the typical module in $T_{n-i}$ given by the cup diagram of $L$ with all $\vee$ 's removed. Applying $D S^{i}$ to any other irreducible module in the same block will result in the same typical weight. Following Serganova [Ser10] we call this unique irreducible module the core of the block $L^{\text {core }}$. Hence $D S^{i}(L)=m(L) \cdot L^{\text {core }} \oplus m^{\prime}(L) \cdot \Pi L^{\text {core }}$. Since the positive integers $m$ and $m^{\prime}$ only depend on the nesting structure of the cup diagram $\underline{\lambda}$, we may compute them in the maximally atypical case. By a comparison with the maximal atypical case $\mathcal{R}_{i}$-case either $m$ or $m^{\prime}$ is zero. As in the maximally atypical case a parity shift happens in $D S(L(\lambda))$ if and only if $\varepsilon(\lambda) \not \equiv \varepsilon\left(\lambda_{i}\right) \bmod 2$. Hence

$$
m(\lambda)= \begin{cases}m & \varepsilon(\lambda) \equiv 0 \bmod 2 \\ m^{\prime} & \varepsilon(\lambda) \equiv 1 \bmod 2 .\end{cases}
$$

This shows that the trace $t_{L}$ does not vanish: Indeed

$$
t_{L}\left(i d_{L}\right):=t_{D S^{i}(L)}^{p}\left(i d_{D S^{i}(L)}\right)=m(\lambda) t_{L^{\text {core }}}^{P}\left(i d_{L^{\text {core }}}\right) \neq 0
$$

since $t^{P}$ is nontrivial.

Using our particular choice for $\operatorname{sdim}_{0}$ on $I_{0}=\operatorname{Proj}$, we define the normalized modified superdimension as

$$
\begin{aligned}
\operatorname{sdim}_{i}(L(\lambda)) & =\operatorname{sdim}_{0}\left(D S^{i}(L)\right)=\operatorname{sdim}_{0}\left(m L^{\text {core }} \oplus m^{\prime} \Pi L^{\text {core }}\right) \\
& =(-1)^{\varepsilon(\lambda)} m(\lambda) \operatorname{sdim}_{0}\left(L^{\text {core }}\right)
\end{aligned}
$$

In particular the modified super dimension does not vanish. Consider for example the irreducible 4-fold atypical representation in $\mathcal{R}_{6}$ with cup diagram 


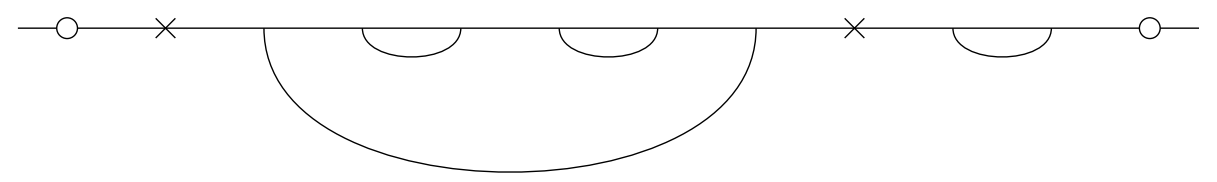

We have already seen above that $m(\lambda)=8$ in this case. The core is given by the typical representation $L(3,-4 \mid 5,-5)$.

As a consequence of our construction and the sign rule of the main theorem we get

Corollary 16.2. If $L$ is irreducible of atypicality $k$, then $\operatorname{sdim}_{k}(L)=$ $\operatorname{sdim}_{k-1}(D S(L))$. If $\operatorname{sdim}_{k}(L)>0$, then all summands in $D S(L)$ have $\operatorname{sdim}_{k-1}(L)>0$.

We can now copy the proof of proposition 8.1 to get

Corollary 16.3. For irreducible atypical objects $L$ in $T_{n}$ the Leray type spectral sequence degenerates:

$$
D S_{n, n_{2}}(L) \cong D S_{n_{1}, n_{2}}\left(D S_{n, n_{1}}(L)\right) \text {. }
$$

\section{StRATEgy OF THE PROOF}

We have already proved the Main Theorem for the groundstates of each block. Recall that a groundstate is a weight with completely nested cup diagram such that all the vertices labelled $\times$ or $\circ$ are to the right of the cups. In the maximally atypical case the ground state are just the Berezinpowers. In the lower atypical cases every ground state is a Berezin-twist of a mixed tensor and we have already seen that these satisfy the main theorem in section 14. The proof of the general case will be a reduction to the case of groundstates.

In the singly atypical case we just have to move the unique label $\vee$ to the left of all of the crosses and circles. We will see in section 18 that we can always move $\vee$ 's to the left of o's or $\times$. The proof of the general case will induct on the degree of atypicality, hence we will always assume that the theorem is proven for irreducible modules of lower atypicality. Hence for the purpose of explaining the strategy of the proof we will focus on the maximally atypical case.

The modules $S^{i}$. Let us consider the following special maximally atypical case. Let $B e r \simeq[1, \ldots, 1] \in \mathcal{R}_{n}$ be the Berezin representation. Let $S^{i}$ denote the irreducible representation $[i, 0, \ldots, 0]$. Every $S^{i-1}$ occurs as the socle and 
cosocel of a mixed tensor denoted $\mathbb{A}_{S^{i+1}}$ [Hei14]. The Loewy structure of the modules $\mathbb{A}_{S^{i}}:=R\left((i),\left(1^{i}\right)\right) \in \mathcal{R}_{n}$ is the following:

$$
\mathbb{A}_{S^{i}}=\left(S^{i-1}, S^{i} \oplus S^{i-2}, S^{i-1}\right)
$$

for $i \neq n$ and $i \geq 1$ and $n \geq 2$ where we use $S^{-1}=0$. Furthermore

$$
\mathbb{A}_{S^{n}}=\left(S^{n-1}, S^{n} \oplus \operatorname{Ber}^{-1} \oplus S^{n-2}, S^{n-1}\right) .
$$

We saw in 14 that for all mixed tensors $D S\left(R\left(\lambda^{L}, \lambda^{R}\right)\right)=R\left(\lambda^{L}, \lambda^{R}\right)$ holds, so we have $D S\left(\mathbb{A}_{S^{i}}\right)=\mathbb{A}_{S^{i}}$ for all $i \geq 1$. Notice that by abuse of notation we view $S^{i}$ and also $\mathbb{A}_{S^{i}}$ as objects of $\mathcal{R}_{n}$ for all $n$.

The image $S^{i} \mapsto D S\left(S^{i}\right)$ can be computed recursively from the two exact sequences in $\mathcal{R}_{n}$

$$
\begin{aligned}
& 0 \longrightarrow K_{n}^{i} \longrightarrow \mathbb{A}_{S^{i}} \stackrel{p}{\longrightarrow} S^{i-1} \longrightarrow 0 \\
& 0 \longrightarrow S^{i-1} \stackrel{j}{\longrightarrow} K_{n}^{i} \longrightarrow S^{i} \oplus ? \oplus S^{i-2} \longrightarrow 0
\end{aligned}
$$

induced by projection $p$ onto the cosocle and the inclusion $j$ of the socle. According to the main theorem we should get for $n \geq 2\left(B e r_{n}\right)_{x}=\Pi B e r_{n-1}$ and

(1) $D S\left(S^{i}\right)=S^{i}$ for $i<n-1$,

(2) $D S\left(S^{i}\right)=S^{i} \oplus \Pi^{n-1-i} \mathrm{Ber}^{-1}$ for $i \geq n-1$.

We proof this for $i \leq n-1$. First notice $H^{-}\left(\mathbb{A}_{S^{i}}\right)=0$ and $H^{+}\left(\mathbb{A}_{S^{i}}\right)=\mathbb{A}_{S^{i}}$. Suppose $i \leq n-1$ and that $H^{-}\left(S^{j}\right)=0, H^{+}\left(S^{j}\right)=S^{j}$ already holds for $j<i$ by induction. This is justified since $S^{0}=k$ equals the trival module. Then the exact hexagons give

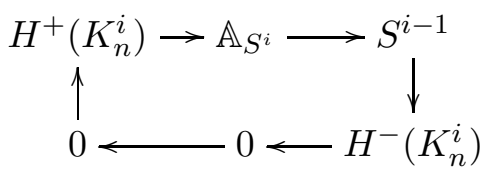

and

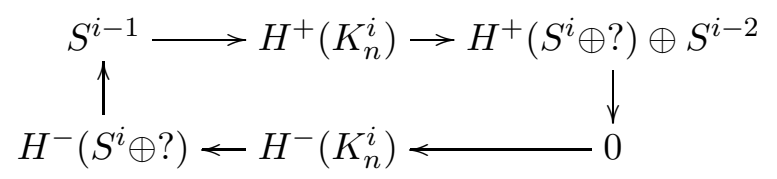

If $H^{+}(p)=0$, then $H^{+}\left(K_{n}^{i}\right) \cong \mathbb{A}_{S^{i}}$. Hence $H^{+}\left(K_{n}^{i}\right) \rightarrow H^{+}\left(S^{i} \oplus\right.$ ? $) \oplus$ $S^{i-2}$ composed with the projection to $S^{i-2}$ is zero, since the cosocle of $H^{+}\left(K_{n}^{i}\right) \cong \mathbb{A}_{S^{i}}$ is $S^{i-1}$. This implies $S^{i-1}=0$, which is absurd. Hence $H^{+}(p)$ is surjective. Therefore $H^{-}\left(K_{n}^{i}\right)=0$ and $H^{+}\left(K_{n}^{i}\right)=K_{n-1}^{i}$, and in particular then

$$
H^{+}\left(K_{n}^{i}\right)=K_{n-1}^{i}
$$


is indecomposable. Hence $K_{n-1}^{i} \rightarrow H^{+}\left(S^{i} \oplus\right.$ ?) $\oplus S^{i-2}$ is surjective, and $H^{-}\left(S^{i}\right)=0$. Furthermore

$$
H^{+}\left(S^{i}\right)=S^{i} \quad, \quad i<n-1
$$

and

$$
H^{+}\left(S^{i}\right)=S^{i} \oplus \operatorname{Ber}^{-1} \quad, \quad i=n-1 .
$$

The proof for the cases $i \geq n$ is similar.

The method described in the $S^{i}$-case doesn't work in general. In the general case we do not have exact analogs of the $\mathbb{A}_{S^{i}}$ - mixed tensors with the property $D S(\mathbb{A})=\mathbb{A}$. In section 18 we associate to every irreducible module three representations, the weight $L$, the auxiliary representation $L^{\text {aux }}$ and the representation $L^{\times \circ}$ and an indecomposable rigid module $F_{i}\left(L^{\times 0}\right)$ of Loewy length 3 with Loewy structure $(L, A, L)$ such that the irreducible module we started with and which we denote $L^{u p}$ for reasons to be explained later is one of the composition factors of $A$. If we apply this construction to irreducible modules of the form $S^{i}=[i, 0, \ldots, 0]$ we recover the modules $\mathbb{A}_{S^{i}}$. Our aim is to use these indecomposable modules as a replacement for the modules $\mathbb{A}_{S^{i}}$.

In the $S^{i}$-case we reduced the computation of $D S\left(S^{i}\right)$ by means of the indecomposable modules $\mathbb{A}_{S^{i}}$ to the trivial case $D S(\mathbf{1})=\mathbf{1}$. In the general case we will reduce the computation of $D S(L)$ by means of the indecomposable modules $F_{i}\left(L^{\times 0}\right)$ to the case of ground states. For that we define an order on the set of cup diagrams for a fixed block such that the completely nested cup diagrams (for which the Main Theorem holds) are the minimal elements. We prove the general case by induction on this order and will accordingly assume that the main theorem holds for all irreducible modules of lower order then a given module $L$. The key point is that for a given module $L^{u p}$ we can always choose our weights $L^{\text {aux }}$ and $L^{\times \circ}=F_{i}\left(L\left(\lambda_{\times \circ}\right)\right)$ such that all other composition factors of $F_{i}\left(L^{\times 0}\right)$ are of lower order then $L^{u p}$. Hence the Main Theorem holds for all composition factors of $F_{i}\left(L^{\times 0}\right)$ except possibly $L^{u p}$. This setup is similar to the $\mathbb{A}_{S^{i}}$-case where we assumed by induction on $i$ that the Main Theorem held for all composition factors of $\mathbb{A}_{S^{i}}=\left(S^{i-1}, S^{i-2}+S^{i}, S^{i-1}\right)$ except possibly $S^{i}$.

Unlike the $\mathbb{A}_{S^{i}}$ the indecomposable modules $F_{i}\left(L^{\times 0}\right)$ are not mixed tensors and hence we do not know a priori their behaviour under $D S$. However assuming that the Main Theorem holds for all composition factors except possibly $L^{u p}$ we prove in section 18 a formula for $D S\left(F_{i}\left(L^{\times \circ}\right)\right)$. In section 19 we show that under certain axioms on the modules $F_{i}\left(L^{\times 0}\right)$ and their image under $D S$ the module $D S\left(L^{u p}\right)$ is semisimple. These axioms are verified in section 20 . Here it is very important that we can control the composition factors of the $F_{i}\left(L^{\times 0}\right)$. The composition factors in the middle 
Loewy layer will be called moves since they can be obtained from the labelled cup diagram of $L$ by moving certain $\vee$ 's in a natural way. The moves are described in detail in section 18.

We still have to explain how the induction process works, i.e. how we relate a given irreducible module to irreducible modules with lesser number of segments respectively sectors. This is done by the so-called Algorithms I and II described first in [Wei10]. As above for a given module $L^{u p}$ all other composition factors of $F_{i}\left(L^{\times 0}\right)$ are of lower order then $L^{u p}$. For $L^{u p}$ with more then one segment we can choose $i$ and the representations $L^{\text {aux }}$ and $L^{\times \circ}$ in such a way that all composition factors have one segment less then $L^{u p}$. We can now apply the same procedure to all the composition factors of $F_{i}\left(L^{\times 0}\right)$ with more then one segment - i.e. we choose for each of these (new) weights $L^{a u x}$ and $L^{\times 0}$ such that the composition factors of the (new) associated indecomposable modules have less segments then them. Iterating this we finally end up with a finite number of indecomposable modules where all composition factors have weight diagrams with only one segment. This procedure is called Algorithm I. In Algorithm II we decrease the number of sectors in the same way: If we have a weight with only one segment but more then one sector we can choose $i$ and the weights $L^{\text {aux }}$ and $L^{\times \circ}$ such that the composition factors of $F_{i}\left(L^{\times 0}\right)$ have less sectors then $L^{u p}$. Applying this procedure to the composition factors of $F_{i}\left(L^{\times 0}\right)$ and iterating we finally relate the cup diagram of $L^{u p}$ to a finite number of cup diagrams with only one sector.

Hence after finitely many iterations we have reduced everything to irreducible modules with one segment and one sector. This sector might not be completely nested, e.g. we might end up with weights with labelled cup diagrams of the type

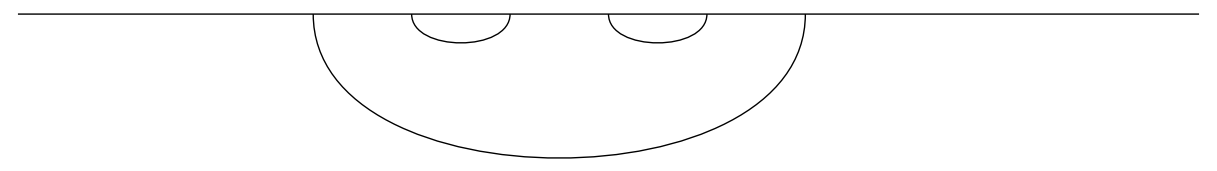

In this case we can apply Algorithm II to the internal cup diagram having one segment enclosed by the outer cup. If we iterate this procedure we will finally end up in a collection of Kostant weights (i.e. weights with completely nested cup diagrams) of this block.

We still have to find the decomposition of the semisimple module $D S\left(L^{u p}\right)$ into its simple summands. Since we know the semisimplicity, we can compute $D S\left(L^{u p}\right)$ on the level of Grothendieck groups. Essentially we compute 
this in the following way: using the notation $\mathbb{A}=F_{i}\left(L\left(\lambda_{\times 0}\right)\right)$, we compute

$$
d(\mathbb{A})=H^{+}(\mathbb{A})-H^{-}(\mathbb{A})=2 d(L)+d(A)=2 d(L)+d\left(L^{u p}\right)+d\left(A-L^{u p}\right)
$$

in $K_{0}\left(\mathcal{R}_{n-1}\right)$ where we do not know $d\left(L^{u p}\right)$ and compare this to the known composition factors of $\tilde{\mathbb{A}}=D S(\mathbb{A})$. For this we need the so-called commutation rules for Algorithm I and Algorithm II. Using that the main theorem holds for all composition factors of $\mathbb{A}$ except possibly $L^{u p}$ we can cancel most composition factors. The remaining factors have to be the simple factors of $D S\left(L^{u p}\right)$ and these factors are exactly those given by the derivative of $L^{u p}$ (seen as a plot), finally proving the theorem. This is done in section 19.

The case $[2,2,0]$. We illustrate the above strategy with an example. In this part we ignore systematically all signs or parity shifts. The module $[2,2,0]$ has the labelled cup diagram

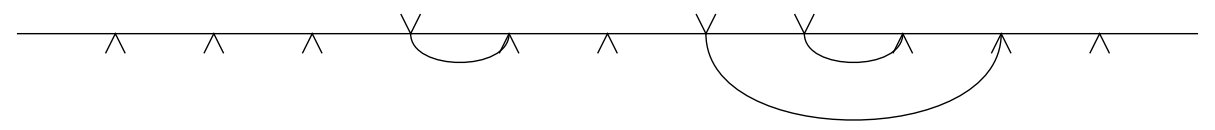

hence it has two segments and two sectors. We will associate to $[2,2,0]$ an auxiliary weight $L$ and a twofold atypical weight $L^{\times \circ}$ in $T_{3}$ such that $[2,2,0]$ is of the form $L^{u p}$ in the indecomposable module $F_{i}\left(L^{\times \circ}\right)$. The auxiliary weight is in this case $[2,1,0]$ with labelled cup diagram

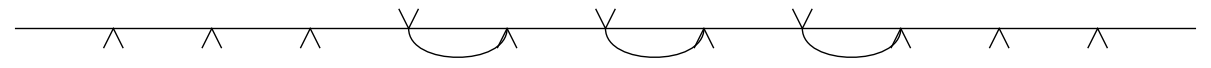

with one segment and three sectors. The weight $\lambda_{x_{0}}$ is obtained from $[2,1,0]$ by replacing the $\vee \wedge$ at the vertices 0 and 1 by $\times \circ$

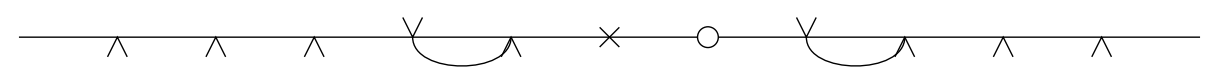

The module $F_{0}\left(L^{\times 0}\right)$ is $*$-selfdual of Loewy length 3 and socle and cosocle $[2,1,0]$. It contains the module $[2,2,0]$ with multiplicity 1 in the middle Loewy layer. The rules of section 18 give the following composition factors (moves) in the middle Loewy layer. In the labelled cup diagram of $[2,1,0]$ there is one internal upper sector $[2,3]$. The internal upper sector move gives the labelled cup diagram

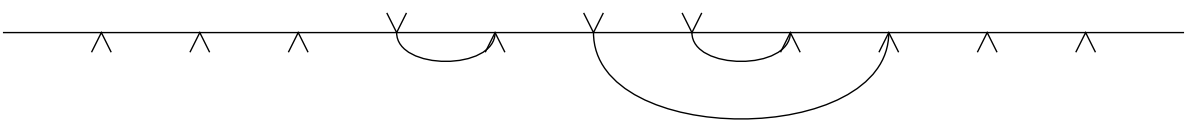

hence the composition factor $[1,1,0]$. The labelled cup diagram of $[2,1,0]$ has one internal lower sector, namely the interval $[-2,-1]$. The associated internal lower sector move gives the labelled cup diagram 


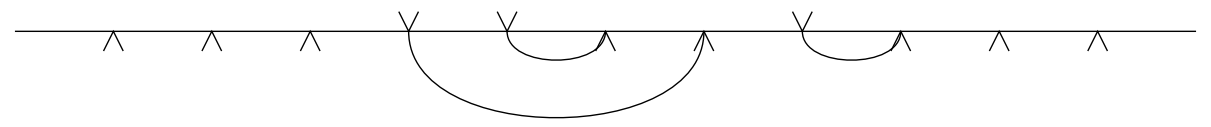

The sector $[0,1]$ is unencapsulated, it is in the middle of the segment $[-2,3]$. Hence we also have the unencapsulated boundary move, i.e. we move the $\vee$ at the vertex 0 to the vertex -3 , resulting in the labelled cup diagram

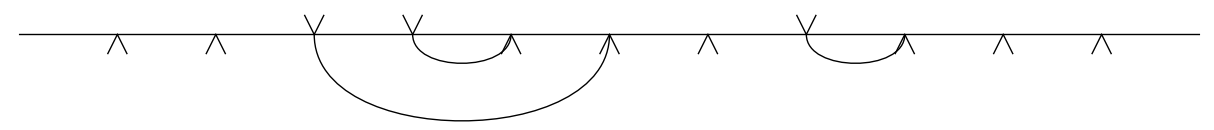

giving the composition factor $[2,-1,-1]$. The upward move of $[2,1,0]$ gives the composition factor $L^{u p}=[2,2,0]$. Hence the Loewy structure of the indecomposable module $F_{0}\left(L^{\text {aux }}\right)$ is

$$
\left(\begin{array}{c}
{[2,1,0]} \\
{[2,-1,-1]+[1,1,0]+[2,0,0]+[2,2,0]} \\
{[2,1,0]}
\end{array}\right) .
$$

We remark that all the composition factors have only one segment, hence we will not have to apply Algorithm I any more. Since the proof inducts on the degree of atypicality we know $D S\left(L^{\times 0}\right)$ and we can apply 18.5 to conclude $D S\left(F_{i}\left(L^{\times \circ}\right)\right)=F_{i}\left(D S\left(L^{\times \circ}\right)\right)=F_{i}\left(L_{1} \oplus L_{2}\right)$ for two irreducible module obtained by applying $D S$ to $L^{\times \circ}$. By the main theorem $D S\left(L^{\times \circ}\right)$ gives the modules

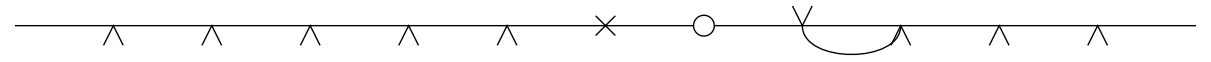

and

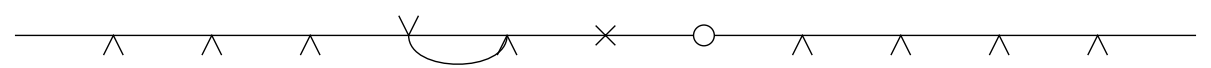

Applying $F_{0}$ to the first summand gives the module $\mathbb{A}_{1}$ with socle and cosocle $[2,1]$. The upward move gives the composition factor $[2,2]$. The unique internal upper sector move gives the composition factor $[1,1]$. We do not have any lower sector moves. The non-encapsulated boundary move gives the composition factor $[2,0]$. This results in the Loewy structures of $\mathbb{A}_{1}=F_{0}\left(L_{1}\right)$ and $A_{2}=F_{0}\left(L_{2}\right)$

$$
\mathbb{A}_{1}=\left(\begin{array}{c}
{[2,1]} \\
{[1,1]+[2,0]} \\
{[2,1]}
\end{array}+[2,2]\right), \quad \mathbb{A}_{2}=\left(\begin{array}{c}
{[0,-1]} \\
{[1,-1]+[-1,-1]+[-2,-2]} \\
{[0,-1]}
\end{array}\right) .
$$

The irreducible modules in the middle Loewy layers give the module $\tilde{A}$. We compare $\tilde{A}$ and $A^{\prime}$ in $K_{0}$ : Taking the derivative of $A=[2,-1,-1]+$ 


$$
\begin{aligned}
{[1,1,0]+[2,0,0] } & +[2,2,0] \text { gives } \\
A^{\prime} & =[2,-1]+[-2,-2]+[1,-1]+[1,1]+[2,0] \\
& +[-1,-1]+[2,-1]+[2,2]
\end{aligned}
$$

with the module $[2,-1]=L^{\text {aux }}$ appearing twice. The computation above of $\mathbb{A}_{1}$ and $\mathbb{A}_{2}$ gives

$$
\tilde{A}=[-2,-2]+[1,-1]+[1,1]+[2,0]+[-1,-1]+[2,2] .
$$

This shows the following commutation rule in this example

$$
A^{\prime}=\tilde{A}+2(-1)^{i+n} L^{a u x} \quad \text { in } \quad K_{0}\left(\mathcal{R}_{n-1}\right) .
$$

We remark that the composition factors $[2,0]$ in $\mathbb{A}_{1}$ and $[-1,-1]$ are detecting objects in the sense of section 20 .

We will prove in section 20 that the properties of the modules $\mathbb{A}, \mathbb{A}_{1}$ and $\mathbb{A}_{2}$ imply that $D S\left(L^{u p}\right)$ is semisimple. Hence we can compute $D S\left(L^{u p}\right)$ by looking at $K_{0}$.

In Algorithm II we reduce everything to a single sector. Take one of the composition factors of $F_{0}\left(L^{\times \circ}\right)$ with more then one sector, eg. [2,1,0] with one segment and three sectors. The associated auxiliary weight is in this case the weight $[2,0,0]$ with the twofold atypical weight $L^{\times \circ}$ given by the labelled cup diagram

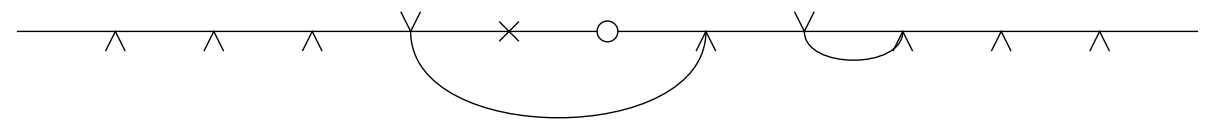

The module $F_{-1}\left(L^{\times 0}\right)$ has socle and cosocle $[2,0,0]$ and the followowing modules in the middle Loewy layer: The upward move gives $[2,1,0]$ and the upper sector move of the upper sector $[2,3]$ gives the weight $[0,0,0]$.

There are no non-encapsulated boundary moves and no internal lower sector moves, hence we get the Loewy structure

$$
F_{-1}\left(L^{\times \circ}\right)=\left(\begin{array}{c}
{[2,0,0]} \\
{[0,0,0]+[2,1,0]} \\
{[2,0,0]}
\end{array}\right) .
$$

We compute $D S\left(F_{-1}\left(L^{\times \circ}\right)\right)$ (using $D S\left(F_{i}\left(L^{\times \circ}\right)\right)=F_{i}\left(D S\left(L^{\times \circ}\right)\right)$ ) (lemma 18.5). By the main theorem $D S\left(L^{\times \circ}\right)$ splits into two direct summands.

Applying $F_{-1}$ to the first and second summand gives the indecomposable modules

$$
\mathbb{A}_{1}=\left(\begin{array}{c}
{[2,0]} \\
{[2,1]+[2,-1]+[0,0]} \\
{[2,0]}
\end{array}\right), \quad \mathbb{A}_{2}=\left(\begin{array}{c}
{[-1,-1]} \\
{[0,-1]} \\
{[-1,-1]}
\end{array}\right)
$$


We remark that all the factors in the middle Loewy layers are detecting objects in the sense of section 19. As shown in section 19 these properties already imply that $D S([2,1,0])$ is semisimple. To compute it we need the commutation rules for Algorithm II, i.e. we compare the derivative $A^{\prime}$ of the middle Loewy layer of $F_{-1}\left(L^{\times 0}\right)$ with the modules $\tilde{A}=A_{1}+A_{2}$ in the middle Loewy layers of $\mathbb{A}_{1}$ and $\mathbb{A}_{2}$. In both cases we get $[2,1]+[2,-1]+$ $[0,0]+[0,-1]$, hence the commutation rule

$$
\tilde{A}=A^{\prime} \text {. }
$$

The general case is proven in lemma 20.7.

\section{Modules of Loewy Length 3}

As described in section 17 we reduce the main theorem to the case of ground states by means of translation functors $F_{i}(\ldots)$. In this section we describe the Loewy layers and composition factors of the objects $F_{i}\left(L_{\times 0}\right)$ and study their behaviour under $D S$.

Khovanov algebras. We review some facts from the articles by Brundan and Stroppel [BS11], [BS10a], [BS12a], [BS12b]. We denote the Khovanovalgebra of [BS12a] associated to $G l(m \mid n)$ by $K(m, n)$. These algebras are naturally graded. For $K(m, n)$ we have a set of weights or weight diagrams which parametrise the irreducible modules (up to a grading shift). This set of weights is again denoted $X^{+}$. For each weight $\lambda \in X^{+}$we have the irreducible module $L(\lambda)$, the indecomposable projective module $P(\lambda)$ with top $L(\lambda)$ and the standard or cell module $V(\lambda)$. If we forget the grading structure on the $K(m, n)$-modules, the main result of [BS12a] is:

Theorem 18.1. There is an equivalence of categories $E$ from $\mathcal{R}_{m \mid n}$ to the category of finite-dimensional left- $K(m, n)$-modules such that $E L(\lambda)=$ $L(\lambda), E P(\lambda)=P(\lambda)$ and $E K(\lambda)=V(\lambda)$ for $\lambda \in X^{+}$.

$E$ is a Morita equivalence, hence $E$ will preserve the Loewy structure of indecomposable modules. This will enable us to study questions regarding extensions or Loewy structures in the category of Khovanov modules. We will use freely the terminology of [BS11], [BS10a], [BS12a], [BS12b]. The notion of cups, caps, cup and cap diagrams are introduced in [BS11]. For the notion of matching between a cup and a cap diagram see [BS10a], section 2. For the notion of $\Gamma$-admissible see [BS12a], section 2.

Let $\lambda$ in $\mathcal{R}_{n}$ be any atypical weight with a $\vee \wedge$-pair in its weight diagram, i.e. such that there exists an index $i$ labelled by $\vee$ and the index $i+1$ is labelled by $\wedge$. Fix such an index $i$ and replace $(\vee \wedge)$ by the labelling $(\times, \circ)$. This defines a new weight $\lambda_{x_{\circ}}$ of atypicality atyp $(\lambda)-1$. We denote by $F_{i}$, 
$i \in \mathbf{Z}$, the endofunctor from [BS12a], (2.13). The functor $F_{i}$ has an avatar $F_{i}$ on the side of Khovanov-modules. This projective functor $F_{i}$ is defined by $F_{i}:=\bigoplus K_{\left(\Gamma-\alpha_{i}\right) \Gamma}^{t_{i}(\Gamma)} \otimes_{K}-$, see [BS12a], (2.3), for summation rules and also [BS10a], (4.1). Since by loc. cit. lemma 2.4, $F_{i} L\left(\lambda_{\times 0}\right)$ is indecomposable, $F_{i} L\left(\lambda_{\times_{0}}\right)=K_{\left(\Gamma-\alpha_{i}\right) \Gamma}^{t_{i}(\Gamma)} \otimes_{K}$ - for one specific $i$-admissible $\Gamma$

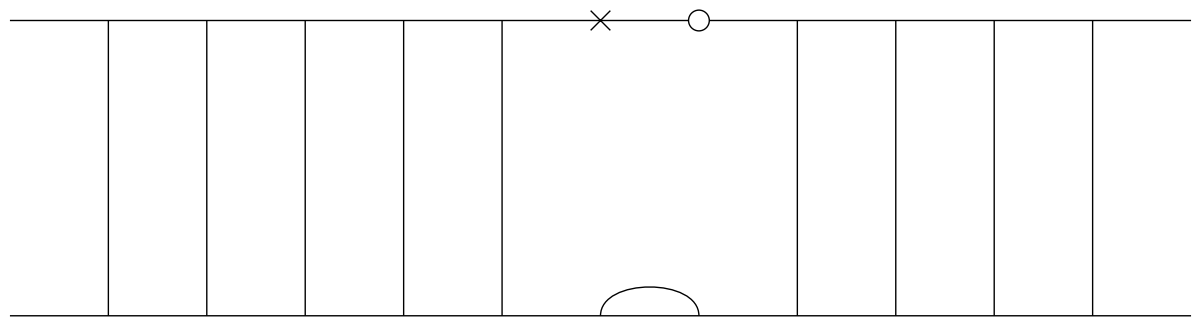

Here the matching between $\left(\Gamma-\alpha_{i}\right)$ and $\Gamma$ is given by the diagram above and the rule that all other vertices, except those labelled by $\times$ or $\circ$, are connected by a vertical identity line segment. We want to determine its composition factors and Loewy layers. For that one considers the modules $F_{i} L\left(\lambda_{\times \circ}\right)$ as modules in the graded category of $K=K(n, n)$-modules where $K(n, n)$ is the Khovanov algebra from [BS12a]. We recall some facts from [BS11] and [BS12a], see also [Hei14].

Let $\Lambda$ be any block in the category of graded $K$-modules. For a graded $K$-module $M=\bigoplus_{j \in \mathbf{Z}} M_{j}$, we write $M\langle j\rangle$ for the same module with the new grading $M\langle j\rangle_{i}:=M_{i-j}$. Then the modules $\{L(\lambda)\langle j\rangle \mid \lambda \in \Lambda, j \in$ $\mathbf{Z}\}$ give a complete set of isomorphism classes of irreducible graded $K_{\Lambda^{-}}$ modules. For the full subcategory $\operatorname{Rep}\left(K_{\Lambda}\right)$ of $\operatorname{Mod}_{l f}\left(K_{\Lambda}\right)$ consisting of finite-dimensional modules, the Grothendieck group is the free $\mathbf{Z}$-module with basis given by the $L(\lambda)\langle j\rangle$. Viewing it as a $\mathbf{Z}\left[q, q^{-1}\right]$-module, so that by definition $q^{j}[M]:=[M\langle j\rangle]$ holds, $K_{0}\left(\operatorname{Rep}\left(K_{\Lambda}\right)\right)$ is the free $\mathbf{Z}\left[q, q^{-1}\right]$-module with basis $\{L(\lambda) \mid \lambda \in \Lambda\}$. We refer to [BS10a], section 2, for the definition of the functors $G_{\Lambda \Gamma}^{t}$. For terminology used in the statement of the next theorem see loc.cit or section 30. We quote from [BS10a], thm 4.11

Theorem 18.2. Let $t$ be a proper $\Lambda \Gamma$-matching and $\gamma \in \Gamma$. Then in the graded Grothendieck group

$$
\left[G_{\Lambda \Gamma}^{t} L(\gamma)\right]=\sum_{\mu}\left(q+q^{-1}\right)^{n_{\mu}}[L(\mu)]
$$

where $n_{\mu}$ denotes the number of lower circles in $\mu$ t and the sum is over all $\mu \in \Lambda$ such that a) $\underline{\gamma}$ is the lower reduction of $\underline{\mu} \bar{t}$ and $b$ ) the rays of each lower line in $\underline{\mu} \mu$ t are oriented so that exactly one is $\vee$ and one is $\wedge$.

Up to a grading shift by - caps $(t)$ we have $F_{i} L\left(\lambda_{\times 0}\right)=G_{\left(\Gamma-\alpha_{i}\right) \Gamma}^{t} L(\gamma)$ for some $\gamma$ and we may apply the theorem above to compute their Loewy 
structure. By [BS12a], lemma 2.4.v, $F_{i} L\left(\lambda_{\times 0}\right)$ is indecomposable with irreducible socle and head isomorphic to $L(\lambda)$.

Proposition 18.3. $F_{i} L\left(\lambda_{\times \circ}\right)$ has a three step Loewy filtration

$$
F_{i} L\left(\lambda_{\times \circ}\right)=\left(\begin{array}{c}
L(\lambda) \\
F \\
L(\lambda)
\end{array}\right)
$$

where all irreducible constituents in (the semisimple) module F occur with multiplicity 1 .

Proof. Let $F(j)$ be the submodule of $F_{i} L\left(\lambda_{\times 0}\right)$ spanned by all graded pieces of degree $\geq j$. Let $k$ be large enough so that all constituents of $F_{i} L\left(\lambda_{\circ \times}\right)$ have degree $\geq-k$ and $\leq k$. Then

$$
F=F(-k) \supset F(-k+1) \supset \ldots \supset F(k)
$$

with successive semisimple quotients $F(j) / F(j+1)$ in degree $j$. In our case we take $k=1$, since the irreducible socle and top $L(\lambda)=L\left(\lambda_{\vee \wedge}\right)$ satisfies $n_{\lambda}=1$. Then all other composition factors $L(\mu)$ necessarily satisfy $n_{\mu}=0$ (we ignore the shift by $\langle-\operatorname{caps}(t)\rangle$ here). The grading filtration thus gives our three step Loewy filtration. The statement about the multiplicity follows since the multiplicity of $L(\mu)$ in $F$ is given by $2^{n_{\mu}}$. The Loewy filtration of $F_{i} L\left(\lambda_{\times \circ}\right)$ is preserved by the Morita equivalence $E^{-1}$ of $K(n, n)-\bmod$ with $\mathcal{R}_{n}$.

Lemma 18.4. $F_{i} L\left(\lambda_{\times \circ}\right)$ is $*$-invariant.

Proof. Since $X \otimes L_{\times, \circ}$ is *-invariant, * permutes its indecomposable summands. The indecomposable summands are either irreducible or are of the form $F_{j} L\left(\lambda_{\times \circ}\right)$ for some $j$ with labeling $(\times, \circ)$ at position $(j, j+1)$. Since * preserves irreducible modules, the indecomposable summands corresponding to the $(\times, \circ)$-pairs in $\lambda_{x \circ}$ are permuted amongst themselves. Since* preserves irreducible modules $\left[M^{*}\right]=[M]$ in $K_{0}$. However all the nonirreducible $F_{j}\left(L\left(\lambda_{\times 0}\right)\right)$ lie in different blocks for $j \neq j^{\prime}$ by the rules of [BS12a], lemma 2.4.

Composition factors. We describe the composition factors of $F_{i}\left(L^{\times 0}\right)$. We can restrict ourselves to the maximally atypical block (i.e. we can ignore $\times$ 's and o's).

Let $\lambda$ be $i$-fold atypical. Since $F_{i}\left(L\left(\lambda_{\times \circ}\right)\right)$ is indecomposable, any highest weight of a composition factor $\mu$ has the same positioning of the $n-i$ crosses and $n-i$ circles as $\lambda$. In particular it has the same positioning of the circles and crosses as $\lambda_{\times \circ}$ except at the position $(i, i+1)$. Let $F_{i}\left(L\left(\lambda_{\times \circ}\right)\right)$ be given by a matching $t$ as follows 


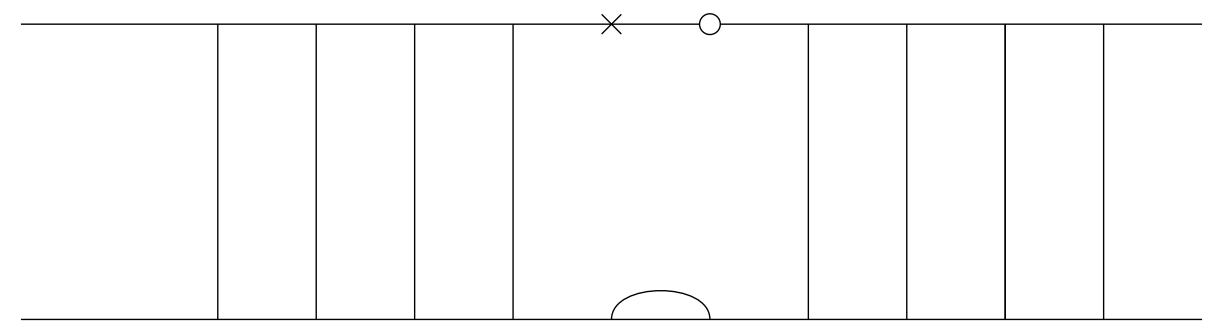

The crosses and the circles are now fixed. Since the composition factors depend only on the nesting structure and the matching $t$ as in theorem 18.2 we can fix them and assume that we are in the maximally atypical block of $G l(i \mid i)$. In this case the composition factors can be determined from the segment and sector structure of $\lambda$ as in [Wei10]. For symbols $x, y \in$ $\{\circ, \wedge, \vee, \times\}$ we write $\lambda_{x y}$ for the diagram obtained from $\lambda$ with the $i$ th and $(i+1)$ th vertices relabeled by $x$ and $y$, respectively.

- Socle and cosocle. They are defined by $L(\mu)$ for $\mu=\lambda_{\vee \wedge}$.

- The upward move. It corresponds to the weight $\mu=\lambda_{\wedge \vee}$ which is obtained from $\lambda_{\vee \wedge}$ by switching $\vee$ and $\wedge$ at the places $i$ and $i+1$. It is of type $\lambda_{\wedge \vee}$.

- The nonencapsulated boundary move. It only occurs in the nonencapsulated case. It moves the $\vee$ in $\lambda_{\vee \wedge}$ from position $i$ to the left boundary position $a$. The resulting weight $\mu$ is of type $\lambda_{\wedge \wedge}$.

- The internal upper sector moves. For every internal upper sector $\left[a_{j}, b_{j}\right]$ (i.e. to the right of $[i, i+1]$ ) there is a summand whose weight is obtained from $\lambda_{\vee \wedge}$ by moving the label $\vee$ at $a_{j}$ to the position $i+1$. These moves define new weights $\mu$ of type $\lambda_{\mathrm{V}}$.

- The internal lower sector moves. For every internal lower sector $\left[a_{j}, b_{j}\right]$ (i.e. to the left of $[i, i+1]$ ) there is a summand whose weight is obtained from $\lambda_{\vee \wedge}$ by moving the label $\vee$ from the position $i$ to the position $b_{j}$. These moves define new weights $\mu$ of type $\lambda_{\wedge \wedge}$.

For examples see [Wei10] or section 17. It follows from the maximal atypical case and the definition of our $\operatorname{sign} \varepsilon(L)$ that we have $F_{i} L\left(\lambda_{\circ \times}\right)=$ $(L, F, L)$ with $L \in \mathcal{R}_{n}( \pm \varepsilon)$ and $F \in \mathcal{R}_{n}(\mp \varepsilon)$. For the following lemma see also [Ser10], thm. 2.1 and cor. 4.4.

Lemma 18.5. Suppose theorem 16.1 holds for the irreducible representation $L^{\times 0}=L\left(\lambda_{\times 0}\right)$ in the block $\Gamma$ of $\mathcal{R}_{n}$. Suppose $i \in \mathbb{Z}$ is $\Gamma$-admissible in the sense of [BS12a], p.6. Then for the special projective functor $F_{i}$ the following holds:

$$
D S\left(F_{i} L_{\times \circ}\right)=F_{i} D S\left(L_{\times \circ}\right) .
$$


Proof. Given $(V, \rho)$ in $\mathcal{R}_{n}$ the Casimir $C_{n}$ of $\mathcal{R}_{n}$ restricts on $D S(V, \rho)$ to the Casimir $C_{n-1}$ of $\mathcal{R}_{n-1}$ by lemma 11.2. On irreducible representations $V$ the Casimir acts by a scalar $c(V)$. Given representations $V_{1}, V_{2}$ in $\mathcal{R}_{n}$, such that $C_{n}$ acts by $c\left(V_{i}\right) \cdot i d_{V_{i}}$ on $V_{i}$, and $v \in V_{1} \otimes V_{2}$, then $C_{n}(v)=$ $\left(c\left(V_{1}\right)+c\left(V_{2}\right)\right) \cdot v+2 \Omega_{n}(v)$ for $\Omega_{n}=\sum_{r, s=1}^{n}(-1)^{\bar{s}} e_{r, s} \otimes e_{s, r} \in \mathfrak{g}_{n} \otimes \mathfrak{g}_{n}$.

Note $F_{i}(V)=p r_{\Gamma-\alpha_{i}} \circ\left(V \otimes X_{s t}\right) \circ p r_{\Gamma}$, so $F_{i} L\left(\lambda_{\times \circ}\right)=p r_{\Gamma-\alpha_{i}}\left(L\left(\lambda_{\times \circ}\right) \otimes\right.$ $\left.X_{s t}\right)$. By [BS12a], lemma 2.10, this is also the generalized $i$-eigenspace of $\Omega_{n}$ on $L\left(\lambda_{\times 0}\right) \otimes X_{s t}$. Put $c=c\left(L\left(\lambda_{\times 0}\right)\right)+c\left(X_{s t}\right)+2 i$. Then $F_{i} L\left(\lambda_{\times 0}\right)$ is the generalized $c$-eigenspace of $C_{n}$ on $L\left(\lambda_{\times 0}\right) \otimes X_{s t}$. Hence $D S\left(F_{i} L\left(\lambda_{\times 0}\right)\right)$ is the generalized $c$-eigenspace of $C_{n-1}$ on $D S\left(L\left(\lambda_{\times \circ}\right) \otimes X_{s t}\right)=D S\left(L\left(\lambda_{\times \circ}\right)\right) \otimes$ $D S\left(X_{s t}\right)=D S\left(L\left(\lambda_{\times \circ}\right)\right) \otimes X_{s t, n-1}$. Observe that $c\left(D S\left(V_{1}\right)\right)+c\left(D S\left(V_{2}\right)\right)=$ $c\left(V_{1}\right)+c\left(V_{2}\right)$, since $C_{n}$ induces $C_{n-1}$ on $D S\left(V_{i}\right)$.

By the main theorem 16.1 (using induction over degree of atypicity) $D S\left(L\left(\lambda_{\times \circ}\right)\right)$ is in a unique block $\bar{\Gamma}$. So $F_{i} D S\left(L\left(\lambda_{\times \circ}\right)\right)=\operatorname{pr}_{\bar{\Gamma}-\alpha_{i}} \circ(? \otimes$ $\left.X_{s t, n-1}\right) \circ \operatorname{pr}_{\bar{\Gamma}} D S\left(L\left(\lambda_{\times \circ}\right)\right)=\operatorname{pr}_{\bar{\Gamma}-\alpha_{i}}\left(D S\left(L\left(\lambda_{\times \circ}\right)\right) \otimes X_{s t, n-1}\right)$, and again by [BS12a], lemma 2.10, this is the generalized $c$-eigenspace of the Casimir $C_{n-1}$ on $D S\left(L\left(\lambda_{\times \circ}\right)\right) \otimes X_{s t, n-1}$. Thus $D S\left(F_{i} L\left(\lambda_{\times \circ}\right)\right) \cong F_{i} D S\left(L\left(\lambda_{\times \circ}\right)\right)$.

Weights, sectors, segments. Let $L(\lambda)$ be $i$-atypical in a block $\Gamma$. Let $X_{\Gamma}^{+}$ denote the set of weights in $\Gamma$. Then we define a map

$$
\phi=\phi_{\Gamma}: X_{\Gamma}^{+} \rightarrow\{\text { plots of rank } i\}
$$

by sending $\lambda$ to the plot of the weight of the irreducible representation $\phi_{n}^{i}(L(\lambda))$. Then $\phi_{\Gamma}$ is a bijection. Each plot has defining segments and sectors, and by transfer with $\phi_{\Gamma}$ this defines the segments and sectors of a given weight diagram in $X_{\Gamma}^{+}$.

Shifting $\times$ and $\circ$. We now quote from [BS12a], lemma 2.4

Lemma 18.6. Let $\lambda \in X^{+}(n)$ and $i \in \mathbb{Z}$. For symbols $x, y \in\{\circ, \wedge, \vee, \times\}$ we write $\lambda_{x y}$ for the diagram obtained from $\lambda$ with the ith and $(i+1)$ th vertices relabeled by $x$ and $y$, respectively.

(i) If $\lambda=\lambda_{\vee \times}$ then $E_{i} L(\lambda) \cong L\left(\lambda_{\times \vee}\right)$. If $\lambda=\lambda_{\times \vee}$ then $F_{i} L(\lambda) \cong$ $L\left(\lambda_{\vee \times}\right)$

(ii) If $\lambda=\lambda_{\wedge \times}$ then $E_{i} L(\lambda) \cong L\left(\lambda_{\times \wedge}\right)$. If $\lambda=\lambda_{\times \wedge}$ then $F_{i} L(\lambda) \cong$ $L\left(\lambda_{\wedge x}\right)$

(iii) If $\lambda=\lambda_{\vee \circ}$ then $F_{i} L(\lambda) \cong L\left(\lambda_{\circ \vee}\right)$. If $\lambda=\lambda_{\circ \vee}$ then $E_{i} L(\lambda) \cong L\left(\lambda_{\vee}\right)$.

(iv) If $\lambda=\lambda_{\wedge \circ}$ then $F_{i} L(\lambda) \cong L\left(\lambda_{\circ \wedge}\right)$. If $\lambda=\lambda_{\circ \wedge}$ then $E_{i} L(\lambda) \cong L\left(\lambda_{\wedge \circ}\right)$.

(v) If $\lambda=\lambda_{\times \circ}$ then: $F_{i} L(\lambda)$ has irreducible socle and head both isomorphic to $L\left(\lambda_{\vee \wedge}\right)$, and all other composition factors are of the form $L(\mu)$ for $\mu \in \lambda$ such that $\mu=\mu_{\vee \wedge}, \mu=\mu_{\wedge \vee}$ or $\mu=\mu_{\wedge \vee}$. Likewise for $\lambda=\lambda_{\circ \times}$ and $E_{i} L(\lambda)$.

(vi) If $\lambda=\lambda_{\vee \wedge}$ then $F_{i} L(\lambda) \cong L\left(\lambda_{\circ \times}\right)$. 
For a pair of neighbouring vertices $(i, i+1)$ in the weight diagram of $\lambda=\lambda_{\vee \times}$, labelled by $(\vee \times)$, we get

$$
E_{i} L\left(\lambda_{\vee \times}\right)=L\left(\lambda_{\times \vee}\right)
$$

from 18.6.1. In other words, the functor replaces the irreducible representation of weight $\lambda_{\times \vee}$ by the irreducible representation of weight $\lambda_{\vee \times}$, which has the same weight diagram as $\lambda_{\times \vee}$, except that the positions of $\times$ and $\vee$ are interchanged. Note that

$$
\phi\left(\lambda_{\vee \times}\right)=\phi\left(\lambda_{\times \vee}\right),
$$

but $L=L\left(\lambda_{\vee \times}\right)$ and $L^{u p}=L\left(\lambda_{\times \vee}\right)$ lie in different blocks.

Lemma 18.7. Suppose for the representation $L=L\left(\lambda_{\vee \times}\right)$ in $\mathcal{R}_{n}^{i}$ the assertion of theorem 16.1 holds. Then it also holds for the representation $L^{u p}=L\left(\lambda_{\times \vee}\right)$.

Proof. By assumption we have a commutative diagram

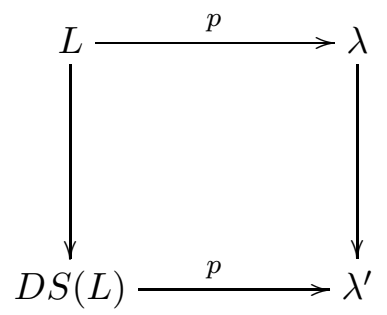

We have to show that we have the same diagram for $L^{u p}$ instead of $L$. Let $S_{\nu}$ denote the sectors of the plot $\lambda=\phi\left(\lambda_{\vee \times}\right)$ and let $S_{j}$ denote the sector containing the integer $p(i)$. Then $D S(L)$ is a direct sum of irreducible representations $L_{\nu}$, whose sector structure either is obtained by replacing one of the sectors $S_{\nu}, \nu \neq j$ by $\partial S_{\nu}$, and there is the unique irreducible summand $L_{j}$ whose sector structure either is obtained by replacing the sectors $S_{j}$ by $\partial S_{j}$. We would like to show that $D S\left(L^{u p}\right)$ can be similarly described in terms of the sector structure of $L^{u p}$. The sectors of $L^{u p}$ literally coincide with the $S_{\nu}$ for $\nu \neq j$, and for $\nu=j$ the remaining sector of $L^{u p}$ is obtained from the sector $S_{j}$ by transposing the positions at the labels $i, i+1$ (within this sector). Hence to show our claim, it remains to show that $D S\left(L^{u p}\right)$ is isomorphic to a direct sum of irreducible representations $L_{\nu}^{u p}$ with the sector structures such that $L_{\nu}^{u p}$ is obtained from $L_{\nu}$ by applying the functor $E_{i}$ (i.e. replacing the positions of $\vee$ and $\times$ at the labels $i, i+1)$. Indeed, the derivative $\partial$ for sectors commutes with the interchange of labels at $i, i+1$ in our situation (the sign rule is obviously preserved). Hence it remains to show

$$
E_{i}\left(D S\left(L\left(\lambda_{\vee \times}\right)\right)\right)=D S\left(E_{i}\left(L\left(\lambda_{\vee \times}\right)\right)\right) .
$$


But this assertion follows by an argument similarly to the one used for the proof of lemma 18.5.

Likewise by lemma 18.6 one can show

Lemma 18.8. Suppose for the representation $L=L\left(\lambda_{\vee_{0}}\right)$ in $\mathcal{R}_{n}^{i}$ the assertion of theorem 16.1 holds. Then it also holds for the representation $L^{u p}=L\left(\lambda_{\circ \vee}\right)$.

Lemma 18.9. Suppose the main theorem holds for the representation $L=L\left(\lambda_{\wedge \times}\right)$ in $\mathcal{R}_{n}^{i}$. Then it also holds for the representation $L^{u p}=L\left(\lambda_{\times \wedge}\right)$.

Lemma 18.10. Suppose the main theorem holds for the representation $L=L\left(\lambda_{\wedge \circ}\right)$ in $\mathcal{R}_{n}^{i}$. Then it also holds for the representation $L^{u p}=L\left(\lambda_{\circ \wedge}\right)$.

\section{InduCTIVE CONTROL OVER $D S$}

We prove now the main theorem under the assumption that there exist objects $\mathbb{A}$ with certain nice properties. Under these assumptions we give an inductive proof of theorem 16.1 using the proposition 19.3 below. We verify in section 20 that certain objects $F_{i}\left(L_{\times \circ}\right)$ verify these conditions.

First recall that for $\varepsilon \in\{ \pm 1\}$ the full abelian subcategories $\mathcal{R}_{n}(\varepsilon)$ of $\mathcal{R}_{n}$ consist of all objects whose irreducible constituents $X$ have sign $\varepsilon(X)=\varepsilon$. We quote from section 16 the following

Proposition 19.1. The categories $\mathcal{R}_{n}(\varepsilon)$ are semisimple abelian categories.

Definition. An object $M$ in $\mathcal{R}_{n}$ is called semi-pure (of sign $\varepsilon$ ), if its socle is in the category $\mathcal{R}_{n}(\varepsilon)$. Every subobject of a semi-pure object is semi-pure. For semi-pure objects $M$ the second layer of the lower Loewy series (i.e. the socle of $M / \operatorname{socle}(M)$ ) is in $\mathcal{R}_{n}(-\varepsilon)$ by the last proposition. Hence by induction, the $i$-th layer of the lower Loewy filtration is in $\mathcal{R}_{n}\left((-1)^{i-1} \varepsilon\right)$. Hence all layers of the lower Loewy filtration are semipure. The last layer $\operatorname{top}(M)$ of the lower Loewy series is semisimple. Since $\operatorname{cosocle}(M) \cong \operatorname{cosocle}(M)^{*} \cong \operatorname{socle}\left(M^{*}\right)$ this easily implies

Lemma 19.2. For semi-pure *-selfdual indecomposable objects $M$ in $\mathcal{R}_{n}$ of Loewy length $\leq 3$ the lower and the upper Loewy series coincide.

We now formulate certain axioms for an object $\mathbb{A}$ of $\mathcal{R}_{n}$. Along with the results of section 18 we will see in section 20 that the translation functors $F_{i}\left(L^{\times 0}\right)$ verify these conditions.

(1) $\mathbb{A} \in \mathcal{R}_{n}$ is indecomposable with Loewy structure $(L, A, L)$.

(2) $\mathbb{A}$ is $*$-selfdual.

(3) $L \in \mathcal{R}_{n}(\varepsilon)$ is irreducible and satisfies theorem 16.1 with $A \in \mathcal{R}_{n}(-\varepsilon)$. 
(4) $\tilde{\mathbb{A}}:=D S(\mathbb{A})=\tilde{\mathbb{A}}^{+} \oplus \Pi\left(\tilde{\mathbb{A}}^{-}\right)$is the direct sum of $\tilde{\mathbb{A}}^{+}:=H^{+}(\mathbb{A})$ and $\tilde{\mathbb{A}}^{-}=H^{-}(\mathbb{A})$ such that $\tilde{\mathbb{A}}^{+}=\bigoplus_{\nu} \tilde{\mathbb{A}}_{\nu}$ and $\tilde{\mathbb{A}}^{-}=\bigoplus_{\bar{\nu}} \tilde{\mathbb{A}}_{\bar{\nu}}$ with indecomposable objects $\tilde{\mathbb{A}}_{\nu} \in \mathcal{R}_{n-1}$ of Loewy structure $\tilde{\mathbb{A}}_{\nu}=\left(\tilde{L}_{\nu}, \tilde{A}_{\nu}, \tilde{L}_{\nu}\right)$ resp. $\tilde{\mathbb{A}}_{\bar{\nu}}, \in \mathcal{R}_{n-1}$ of Loewy structure $\tilde{\mathbb{A}}_{\bar{\nu}}=\left(\tilde{L}_{\bar{\nu}}, \tilde{A}_{\bar{\nu}}, \tilde{L}_{\bar{\nu}}\right)$.

(5) All $\tilde{L}_{\nu}$ and $\tilde{L}_{\bar{\nu}}$ are irreducible so that $\tilde{L}_{\nu} \in \mathcal{R}_{n-1}(\varepsilon)$ and $\tilde{L}_{\bar{\nu}} \in$ $\mathcal{R}_{n-1}(-\varepsilon)$; furthermore $\tilde{A}_{\nu} \in \mathcal{R}_{n-1}(-\varepsilon)$ and $\tilde{A}_{\bar{\nu}} \in \mathcal{R}_{n-1}(\varepsilon)$

(6) For each $\mu=\nu$ (resp. $\mu=\bar{\nu}$ ) there exist irreducible detecting objects

$$
A_{\mu}^{\prime} \subseteq \tilde{A}_{\mu},
$$

also contained in $H^{+}(A)$ (resp. in $H^{-}(A)$ ), such that

$$
\operatorname{Hom}_{\mathcal{R}_{n-1}}\left(A_{\mu}^{\prime}, H^{ \pm}(L)\right)=0 \text { and } \operatorname{Hom}_{\mathcal{R}_{n-1}}\left(A_{\mu}^{\prime}, \bigoplus_{\rho \neq \nu} \tilde{A}_{\rho}\right)=0 .
$$

Remark. For $*$-selfdual indecomposable objects as above the layers (graded pieces) of the upper and lower Loewy filtrations coincide, since otherwise proposition 19.1 would give a contradiction. In the situation above we assume that $\mathbb{A}$ is $*$-selfdual of Loewy length 3 with socle $\operatorname{socle}(\mathbb{A}) \cong L$ and $\operatorname{cosocle}(\mathbb{A}) \cong \operatorname{socle}(\mathbb{A})^{*} \cong L^{*} \cong L$ and middle layer $A$.

Remark. For the later applications we notice that we will construct the detecting objects $A_{\mu}^{\prime}$ in $H^{+}\left(A^{\text {down }}\right)$ (resp. $H^{-}\left(A^{\text {down }}\right)$ ) where $A^{\text {down }}$ will be an accessible summand of $A$. By induction we later will also know that these submodules $A_{\mu}^{\prime}$ therefore already satisfy theorem 16.1. Hence it suffices to check the properties $A_{\mu}^{\prime} \subseteq \tilde{A}_{\mu}$ and $A_{\mu}^{\prime} \subset H^{ \pm}(A)$, since these already imply by the main theorem (valid for summands of $A^{\text {down }}$ ) the stronger assertion made in the axiom telling whether $A_{\mu}^{\prime}$ appears in $H^{+}(A)$ or $H^{-}(A)$. Notice $A_{\mu}^{\prime} \subseteq \tilde{A}_{\mu}$ and $\tilde{A}_{\mu} \in \mathcal{R}_{n-1}(\mp \varepsilon)$ depending on $\mu=\nu$ resp. $\bar{\nu}$. On the other hand $A^{\text {down }} \subset A \in \mathcal{R}_{n}(-\varepsilon)$. Hence, if the main theorem is valid for $A^{\text {down }}$, we get $A_{\mu}^{\prime} \in H^{+}(A)$ for $\mu=\nu$ and $A_{\mu}^{\prime} \in H^{-}(A)$ for $\mu=\bar{\nu}$.

Proposition 19.3. Under the assumptions on $\mathbb{A}$ from above the $H^{ \pm}(A)$ will be semisimple objects in $\mathcal{R}_{n-1}(\mp \varepsilon)$.

We will prove the key proposition 19.3 below after listing some of its consequences.

The ring homomorphism $d$. As an element of the Grothendieck group $K_{0}\left(\mathcal{R}_{n-1}\right)$ we define for a module $M \in \mathcal{R}_{n}$

$$
d(M)=H^{+}(M)-H^{-}(M) .
$$

Notice $d$ is additive by lemma 2.1. Notice

$$
K_{0}\left(T_{n}\right)=K_{0}\left(\mathcal{R}_{n}\right) \oplus K_{0}\left(\Pi \mathcal{R}_{n}\right)=K_{0}\left(\mathcal{R}_{n}\right) \otimes(\mathbb{Z} \oplus \mathbb{Z} \cdot \Pi) .
$$


We have a commutative diagram

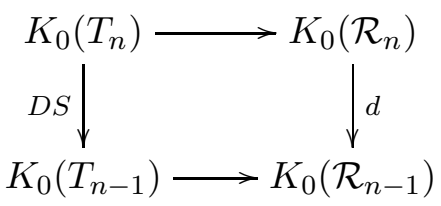

where the horizontal maps are surjective ring homomorphisms defined by $\Pi \mapsto-1$. Since $D S$ induces a ring homomorphism, it is easy to see that $d$ defines a ring homomorphism.

The assertion of the last proposition implies that $H^{+}(A)$ and $H^{-}(A)$ have no common constituents in $\mathcal{R}_{n-1}$ and that they are semisimple. Therefore $d(A)=H^{+}(A)-H^{-}(A) \in K_{0}\left(\mathcal{R}_{n-1}\right)$ uniquely determines $H^{ \pm}(A)$ up to an isomorphism. By the additivity of $d$ and $d(\mathbb{A})=\tilde{\mathbb{A}}$ we get $2 d(L)+d(A)=$ $2 \tilde{L}+\tilde{A}$ in $K_{0}\left(\mathcal{R}_{n-1}\right)$. Hence

Corollary 19.4. $H^{+}(A) \in \mathcal{R}_{n-1}(-\varepsilon)$ and $H^{-}(A) \in \mathcal{R}_{n-1}(\varepsilon)$ are uniquely determined by the following formula in $K_{0}\left(\mathcal{R}_{n-1}\right)$

$$
H^{+}(A)-H^{-}(A)=d(A)=\tilde{A}+2(\tilde{L}-d(L)) .
$$

We later apply this in situations where $\tilde{L}-d(L)=(-1)^{i+n} L^{\text {aux }}$ holds by lemma 20.1 and 20.4 and $A^{\prime}-\tilde{A}=2(-1)^{i+n} L^{\text {aux }}$ holds by lemma 20.3 and 20.7, for some object $L^{\text {aux }}$. Here $A^{\prime}$ denotes the normalized derivative of $A$, introduced in section 15 , defining a homomorphism

$$
': K_{0}\left(\mathcal{R}_{n}\right) \rightarrow K_{0}\left(\mathcal{R}_{n-1}\right)
$$

Hence the last corollary implies the following theorem which repeatedly applied proves theorem 16.1 by induction.

Theorem 19.5. Under the axioms on $\mathbb{A}$ from above $d(A)=H^{+}(A)-$ $H^{-}(A)$ is the derivative $A^{\prime}$ of $A$.

Proof of the proposition 19.3. Step 1). Assumption (4) implies $H^{+}(\mathbb{A})=$ $\tilde{\mathbb{A}}^{+}$and $H^{-}(\mathbb{A})=\tilde{\mathbb{A}}^{-}$in $\mathcal{R}_{n-1}$.

Step 2). Axiom (1) on the Loewy structure of $\mathbb{A}$ therefore gives exact hexagons in $\mathcal{R}_{n-1}$ for $K:=\operatorname{Ker}(\mathbb{A} \rightarrow L)$ using $K / L \cong A$ :

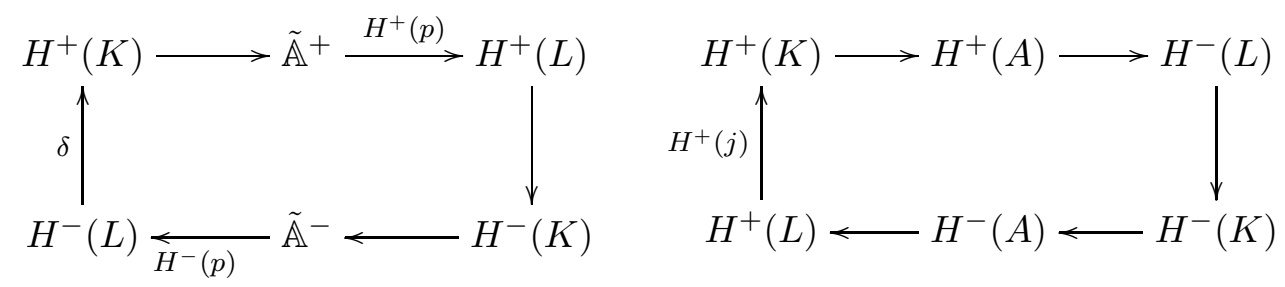


Step 3) Assumption (3),(4),(5) on the Loewy structure of the $\tilde{\mathbb{A}}_{\nu}$ and $H^{ \pm}(L)$ imply the following factorization property for $\tilde{\mathbb{A}}^{+}$(and then similarly also for $\tilde{\mathbb{A}}^{-}$)

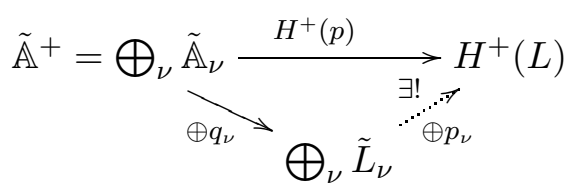

Step 4) Let $\Sigma$ be the set of all $\nu$ such that $p_{\nu}=0$. (Similarly let $\bar{\Sigma}$ be the set of all $\bar{\nu}$ such that $\left.p_{\bar{\nu}}=0\right)$. Then we obtain exact sequences

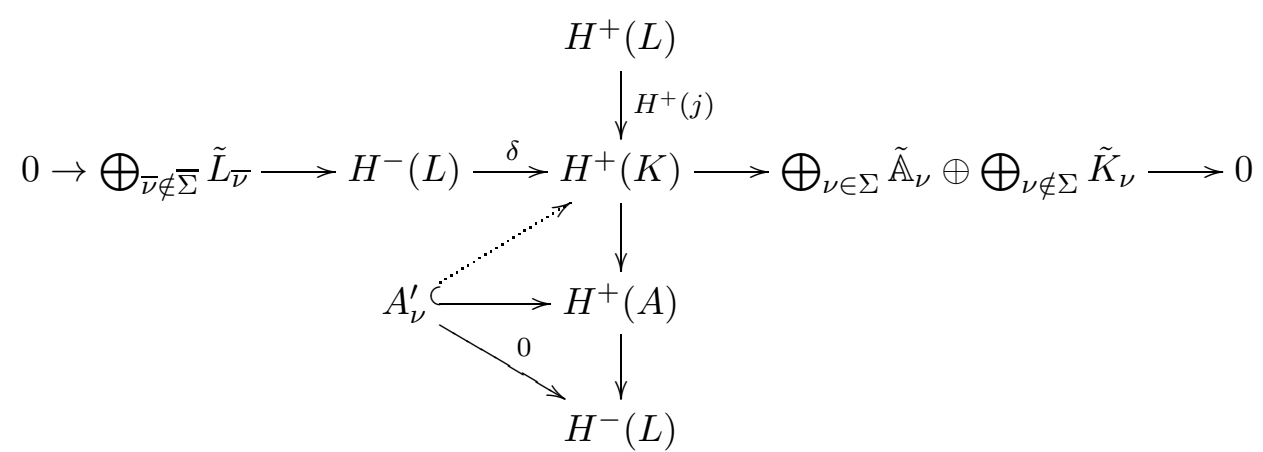

Step 5) The detecting object $A_{\nu}^{\prime} \hookrightarrow H^{+}(A)$ has trivial image in $H^{-}(L)$ by axiom (6), hence can be viewed as a quotient object of $H^{+}(K)$. Again by axiom (6) we can then view $A_{\nu}^{\prime}$ as a nontrivial quotient object of

$$
H^{+}(K) /\left(I+\delta\left(H^{-}(L)\right)\right)
$$

where $I:=H^{+}(j)\left(H^{+}(L)\right)$ is the image of $H^{+}(L)$ in $H^{+}(K)$.

Step 6) The cosocle of $\bigoplus_{\nu \in \Sigma} \tilde{\mathbb{A}}_{\nu} \oplus \bigoplus_{\nu \notin \Sigma} \tilde{K}_{\nu}$ is $\bigoplus_{\nu \in \Sigma} \tilde{L}_{\nu} \oplus \bigoplus_{\nu \notin \Sigma} \tilde{A}_{\nu}$ by assumption (4) on the Loewy structure of $\tilde{\mathbb{A}}_{\nu}, \tilde{K}_{\nu}$.

Step 7) The simple quotient object $A_{\nu}^{\prime}$ of $H^{+}(K)$ can be viewed as a nontrivial quotient object of the cosocle of $H^{+}(K)$ by step 5). We have an exact sequence

$$
H^{-}(L) / \bigoplus_{\overline{\bar{\Sigma}} \overline{L_{\nu}}} \tilde{L}_{\bar{\nu}} \rightarrow \operatorname{cosocle}\left(H^{+}(K)\right) \rightarrow \operatorname{cosocle}\left(\bigoplus_{\nu \in \Sigma} \tilde{\mathbb{A}}_{\nu} \oplus \bigoplus_{\nu \notin \Sigma} \tilde{K}_{\nu}\right) \rightarrow 0
$$

and we can view $A_{\nu}^{\prime}$ as a nontrivial quotient object of

$$
\operatorname{cosocle}\left(\bigoplus_{\nu \in \Sigma} \tilde{\mathbb{A}}_{\nu} \oplus \bigoplus_{\nu \notin \Sigma} \tilde{K}_{\nu}\right)=\bigoplus_{\nu \in \Sigma} \tilde{L}_{\nu} \oplus \bigoplus_{\nu \notin \Sigma} \tilde{A}_{\nu}
$$


by step 5) and 6). Notice that we can consider $A_{\nu}^{\prime}$ for arbitrary $\nu$. For $\nu \in \Sigma$ the last assertion contradicts axiom (6):

$$
\operatorname{Hom}_{\mathcal{R}_{n-1}}\left(\bigoplus_{\mu \in \Sigma} \tilde{L}_{\mu} \oplus \bigoplus_{\mu \notin \Sigma} \tilde{A}_{\mu}, A_{\nu}^{\prime}\right)=0
$$

This contradiction forces

$$
\Sigma=\emptyset \text { and similarly } \quad \bar{\Sigma}=\emptyset,
$$

so we obtain two exact sequences

$$
\begin{aligned}
0 \longrightarrow \bigoplus_{\bar{\nu}} \tilde{L}_{\bar{\nu}} \longrightarrow H^{-}(L) \longrightarrow H^{+}(K) \longrightarrow \bigoplus_{\nu} \tilde{K}_{\nu} \rightarrow 0 \\
0 \longrightarrow H_{\nu} \tilde{L}_{\nu} \longrightarrow H^{+}(L) \longrightarrow \bigoplus_{\bar{\nu}} \tilde{K}_{\bar{\nu}} \rightarrow 0
\end{aligned}
$$

Step 8) The last step 7) proves that

$$
H^{+}(p) \text { is injective on the cosocle } \bigoplus_{\nu} \tilde{L}_{\nu} \text { of } H^{+}(\mathbb{A}) \text {. }
$$

Let $i: L \hookrightarrow \mathbb{A}$ be the composition of $j: L \hookrightarrow K$ and the inclusion $K \hookrightarrow \mathbb{A}$. Then $i: L \hookrightarrow \mathbb{A}$ is the $*$-dual of the projection $p: \mathbb{A} \rightarrow L$ by the axiom (2). Hence by $*$-duality we get from the previous assertion on $H^{+}(p)$ the following assertion

$$
H^{+}(i) \text { surjects onto the socle } \bigoplus_{\nu} \tilde{L}_{\nu} \text { of } H^{+}(\mathbb{A}) .
$$

Now considering

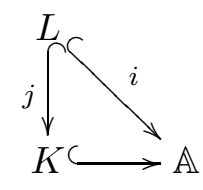

and

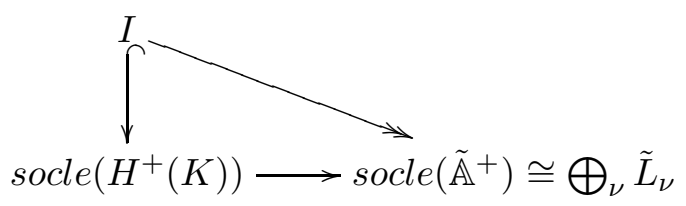

we see that $\bigoplus_{\nu} \tilde{L}_{\nu}$ can also be embedded into the semisimple $I$ as a submodule $\bigoplus_{\nu} \tilde{L}_{\nu} \hookrightarrow I$.

Step 9) Recall the following diagram

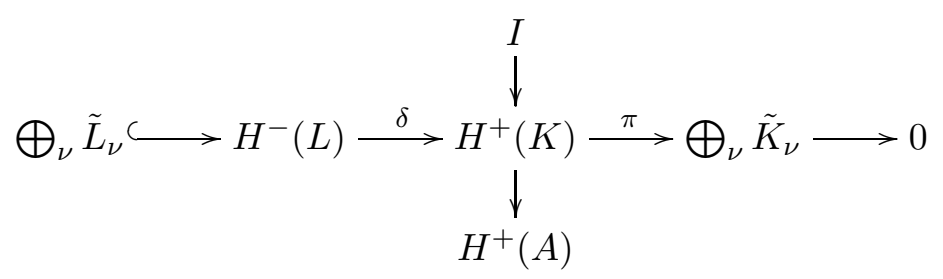

Since $I$ is in $\mathcal{R}_{n-1}(\varepsilon)$ and $H^{-}(L) \in \mathcal{R}_{n-1}(-\varepsilon)$ by our axioms, we also have

$$
\delta\left(H^{-}(L)\right) \cap I=\{0\} \text {. }
$$


Hence the composite of $\pi$ and the inclusion $I \hookrightarrow H^{+}(K)$ maps the semisimple module $I$ injectively into the socle of $\bigoplus_{\nu} \tilde{K}_{\nu}$. Since $\operatorname{socle}\left(\bigoplus_{\nu} \tilde{K}_{\nu}\right)=$ $\bigoplus_{\nu} \tilde{L}_{\nu}$ and since $I$ contains $\bigoplus_{\nu} \tilde{L}_{\nu}$ as a submodule, this implies that

$$
\pi: I \longrightarrow \operatorname{socle}\left(\bigoplus_{\nu} \tilde{K}_{\nu}\right) \cong \bigoplus_{\nu} \tilde{L}_{\nu}
$$

is an isomorphism. Notice $\left(\bigoplus_{\nu} \tilde{K}_{\nu}\right) / \operatorname{socle}\left(\bigoplus_{\nu} \tilde{K}_{\nu}\right) \cong \bigoplus_{\nu} \tilde{A}_{\nu}$.

Step 10) The last isomorphism of step 9) gives the exact sequence

$$
0 \rightarrow \bigoplus_{\bar{\nu}} \tilde{L}_{\nu} \rightarrow H^{-}(L) \rightarrow\left(H^{+}(K) / I\right) \rightarrow \bigoplus_{\nu} \tilde{A}_{\nu} \rightarrow 0
$$

By our assumptions $H^{-}(L)$ is in $\mathcal{R}_{n-1}(-\varepsilon)$, and hence semisimple. Furthermore all $\tilde{A}_{\nu}$ are semisimple and contained in $\mathcal{R}_{n-1}(-\varepsilon)$. Hence by proposition 19.1 $H^{+}(K) / I$ is semisimple and contained in $\mathcal{R}_{n-1}(-\varepsilon)$.

Step 11). By step 10) and the exact hexagon

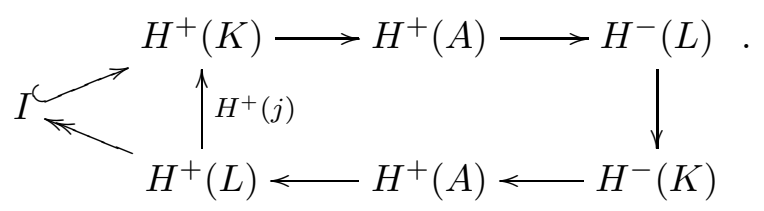

$H^{+}(A)$ defines an extension of the semisimple module $H^{+}(K) / I$ by a submodule of $H^{-}(L)$

$$
0 \rightarrow H^{+}(K) / I \rightarrow H^{+}(A) \rightarrow \operatorname{Ker}\left(H^{-}(L) \rightarrow H^{-}(K)\right) \rightarrow 0 .
$$

Since $H^{+}(K) / I$ and $\operatorname{Ker}\left(H^{-}(L) \rightarrow H^{-}(K)\right)$ are both in $\mathcal{R}_{n-1}(-\varepsilon)$, the proposition 19.1 implies that

$$
H^{+}(A) \cong\left(H^{+}(K) / I\right) \oplus \operatorname{Ker}\left(H^{-}(j): H^{-}(L) \rightarrow H^{-}(K)\right)
$$

is semisimple and contained in $\mathcal{R}_{n-1}(-\varepsilon)$. The first summand has been computed above. Similarly then

$$
H^{-}(A) \cong\left(H^{-}(K) / \bar{I}\right) \oplus \operatorname{Ker}\left(H^{+}(j): H^{+}(L) \rightarrow H^{+}(K)\right)
$$

is semisimple and contained in $\mathcal{R}_{n-1}(\varepsilon)$.

Example. Recall the indecomposable $*$-selfdual objects $\mathbb{A}_{S^{i}}$ in $\mathcal{R}_{n}, n \geq 2$ for $i=1,2, \ldots$ with Loewy structure $(L, A, L)$ where $L=S^{i-1}$ and

$$
A=S^{i} \oplus S^{i-2} \oplus \delta_{n}^{i} \cdot \operatorname{Ber}_{n}^{-1} .
$$

Concerning the notations: $\delta_{n}^{i}$ denotes Kronecker's delta and $S^{-1}=0$. The conditions (1)-(5) are satisfied for $\varepsilon=(-1)^{i-1}$ and $A^{\prime}=S^{i-2}$. Indeed condition (5) follows, since by induction on $i$ one can already assume that $H^{-}(L)=H^{-}\left(S^{i-1}\right)$ is $B e r^{-1}$ or zero and that $H^{+}\left(S^{i-2}\right)$ contains $S^{i-2}$. 
Then by induction on $i$ the computation of $H^{ \pm}(A)$ in terms of $\tilde{A}, \tilde{L}, H^{ \pm}(L)$ from above easily gives as in section 17 the following result

Proposition 19.6. Suppose $n \geq 2$. Then for the functor $D S: \mathcal{R}_{n} \rightarrow T_{n-1}$ of Duflo-Serganova we obtain $D S\left(B e r_{n}\right)=\Pi\left(B e r_{n-1}\right)$ and

(1) $D S\left(S^{i}\right)=S^{i}$ for $i<n-1$,

(2) $D S\left(S^{i}\right)=S^{i} \oplus \Pi^{n-1-i} B_{e r}^{-1}$ for $i \geq n-1$.

\section{Moves}

We verify now the conditions on the indecomposable objects $\mathbb{A}$ of section 19 for the translation functors $F_{i}\left(L\left(\lambda_{\times 0}\right)\right)$. Additionally we verify the commutation rules in and after corollary 19.4. Instead of working directly with the irreducible representation $L$ we use the associated plot as in section 13 and 18. Recall that a plot $\lambda$ is a map $\lambda: \mathbb{Z} \rightarrow\{\boxplus, \boxminus\}$. We also use the notation $\boxplus_{i}$ to indicate that the $\lambda(i)=\boxplus$ and likewise for $\boxminus$. For an overview of the algorithms I and II used in this section see section 17.

Let $L=(I, K)$ for $I=[a, b]$ be a segment with sectors $S_{1}, . ., S_{k}$ from left to right. Suppose $S_{j}=[i, i+1]$ is a sector of rank 1 . Then the segment may be visualized as

$$
L=\left(S_{1} \cdots S_{j-1}\left[\boxplus_{i}, \boxminus_{i+1}\right] S_{j+1} \cdots S_{k}\right) .
$$

We define the upward move of the segment $L$ as the plot defined by the two segments with intervals $[a, i-1]$ and $[i+1, b+1]$

$$
L^{u p}=\left(S_{1} \cdots S_{j-1}\right) \boxminus_{i}\left(\int\left(S_{j+1} \cdots S_{k}\right)\right) .
$$

Similarly we define the downward move of the segment $L$ as the plot defined by the two segments with intervals $[a-1, i]$ and $[i+2, b]$

$$
L^{\text {down }}=\left(\int\left(S_{1} \cdots S_{j-1}\right)\right) \boxminus_{i+1}\left(S_{j+1} \cdots S_{k}\right) .
$$

Furthermore for $r \neq j$ we define additional $r$-th internal lower resp. upper downward moves $L_{r}^{\text {down }}$ by the plots associated ${ }^{1}$ to the single segments

$$
\left(S_{1} \cdots S_{r-1} \int\left(S_{r}^{\prime} \int\left(S_{r+1} \cdots S_{j-1}\right)\right) S_{j+1} \cdots S_{k}\right)
$$

for each $1 \leq r \leq j-1$ respectively

$$
\left(S_{1} \cdots S_{j-1} \int\left(\int\left(S_{j+1} \cdots S_{r-1}\right) S_{r}^{\prime}\right) S_{r+1} \cdots S_{k}\right)
$$

\footnotetext{
${ }^{1}$ For $r=j-1$ or $r=j+1$ the inner integral over the empty sector is understood to give the sector $([i-1, i],\{i-1\})$ respectively $([i+1, i+2],\{i+1\})$.
} 
for each $j+1 \leq r \leq k$. Explaining the notion 'internal', notice that the segments defined by these internal downward moves have the same underlying interval $I=[a, b]$ as the segment $L$ we started from. We remark that the last formulas do remind on partial integration. Formally by setting $L_{r}^{\text {down }}:=L^{\text {down }}$ for $r=j$, we altogether obtain $k$ downward moves and one upward move. All these moves preserve the rank.

The plot $L$ has a sector $[i, i+1]$ of rank 1 . The auxiliary plot $L^{\text {aux }}$ attached to $L$ (and $[i, i+1]$ ) is the plot of rank $r(L)-1$ defined by two segments with intervals $[a, i-1]$ and $[i+2, b]$

$$
L^{\text {aux }}=\left(S_{1} \cdots S_{j-1}\right) \boxminus_{i} \boxminus_{i+1}\left(S_{j+1} \cdots S_{k}\right)
$$

and we also consider

$$
L^{\times \circ}=\left(S_{1} \cdots S_{j-1}\right) \times_{i} \circ_{i+1}\left(S_{j+1} \cdots S_{k}\right) .
$$

Algorithm I (lowering sectors). For a plot with $k$ sectors $S_{\nu}$ with ranks $r_{\nu}=r\left(S_{\nu}\right) \geq 0$ and the distances $d_{\nu} \geq 0$ for $\nu=1, \ldots . k$ (from left to right) we formally define $r_{k+1}=r_{k+1}=\ldots=0$ and $d_{k}=d_{k+1}=\ldots=0$. We can then compare different plots with respect to the lexicographic ordering of the sequences

$$
\left(-r_{1}, d_{1},-r_{2}, d_{2}, \ldots . .\right) \text {. }
$$

Within the set of plots of fixed rank say $n$, the minimum with respect to this ordering is attained if $r_{1}=n$, i.e. if there exists only one sector.

Algorithm I will be applied to given plots, say $\lambda_{\wedge \vee}$, with more than one segment. The upshot is: In this situation one can always find a lexicographic smaller plot $L$ so that the given plot is of the form $\lambda_{\wedge \vee}=L^{u p}$ and such that $L$ and all plots obtained by the moves $L_{r}^{\text {down }}$ of $L$ are strictly smaller than the starting plot $L^{u p}$. Algorithm I is used for induction arguments to reduce certain statements (e.g. theorem 16.1) to the case of plots with 1 segment.

Definition of $L$. For a given plots say $\lambda_{\wedge \vee}$, with more than one segment, $d_{\nu}>0$ holds for some integer $\nu$. So choose $j$ so that the distances $\operatorname{dist}\left(S_{1}, S_{2}\right)=\ldots=\operatorname{dist}\left(S_{j-2}, S_{j-1}\right)=0$ for the sectors $S_{1}, . ., S_{j-1}$ of $\lambda_{\wedge \vee}$ and $\operatorname{dist}\left(S_{j-2}, j-1\right)>0$. We temporarily write $S$ for the next sector $S$ of $\lambda_{\wedge \vee}$. Interpret $S=\int\left(S_{j+1} \cdots S_{k}\right)$ for some sectors $S_{j}, \ldots, S_{k}$. This is possible, but keep in mind that $S_{j}, \ldots, S_{k}$ are not sectors of $\lambda_{\wedge \vee}$ but will be sectors of $L$, and this explains the notation. Indeed, for $i+1=\min (S)$, we define $L$ to be

$$
L=\left(S_{1} \cdots S_{j-1}\right) \ldots d_{j-1} \ldots\left(S_{j} S_{j+1} \cdots S_{k}\right) \ldots d_{k} \ldots
$$

with $S_{j}$ of rank 1 at the positions $[i, i+1]$. To simplify notations we do not write further sectors to the right, since the sectors of $\lambda_{\wedge \vee}$ to the right of $S$ 
will not play an essential role in the following. Indeed, they will appear verbatim in the sector structure of $L$ up to some distance shifts at the following positions

$$
\operatorname{dist}\left(S_{j-1}, S\right)=1+d_{j-1} \quad, \quad \operatorname{dist}(S, \text { next sector })=d_{k}-1 .
$$

Concerning the lexicographic ordering

$$
\operatorname{dist}\left(S_{j-1}, S_{j}\right)=d_{j-1}<\operatorname{dist}\left(S_{j-1}, S\right)=1+d_{j-1}
$$

shows that $L$ is smaller than $L^{u p}=\lambda_{\wedge \vee}$. We leave it to the reader to check that also all $L_{j}^{\text {down }}$ are smaller than $L^{u p}=\lambda_{\wedge \vee}$. Notice, here we apply the moves as in the preceding paragraph with the notable exceptions that

(1) There may be further sectors beyond $S_{k}$. These are just appended, and do not define new moves.

(2) If $d_{j-1} \geq 1$ the sector $S_{j-1}$ has distance $>0$ to the sector $S_{j}$ and therefore does not define downward moves, so that only the doenward moves $L_{r}^{\text {down }}$ for $r=j, \ldots, k$ are relevant.

In the later discussion we always display the more complicated case where $d_{j-1}=0$ (without further mentioning). For the case $d_{j-1}>0$ one can simply 'omit' $S_{1}, \ldots, S_{j-1}$, by just appending them in the same way as we agreed to 'omit' sectors to the right of $S_{k}$.

Construction of detecting objects for algorithm I. Fix $L=L\left(\lambda_{\wedge \vee}\right)$ with the sector $[i, i+1]$. Then $L$ is determined by its sectors. For the construction of detecting objects we are only interested in down moves. In the following it therefore suffices only to keep track of the sectors below $[i, i+1]$ in the segment containing the sector $[i, i+1]$. Notice that $L$ is a union of the sector $[i, i+1]$ and, say $s$, other sectors $S_{\nu}$. Let $S_{1}, . ., S_{j-1}$ denote the sectors below $[i, i+1]$ in the segment of $[i, i+1]$. Hence $L$ is

$$
\boxminus S_{1} \cdots S_{j-1}\left[\boxplus_{i} \boxminus_{i+1}\right]
$$

and the union of other disjoint sectors $S_{\nu}$ for $j+1 \leq \nu \leq s$. Then $L^{\times \circ}$ is

$$
\boxminus S_{1} \cdots S_{j-1}\left[\times_{i} \circ_{i+1}\right]
$$

and the union of other disjoint sectors $S_{\nu}$ for $j+1 \leq \nu \leq s$. We define $\mathbb{A}=F_{i} L^{\times 0}$. Then $\mathbb{A}$ is $*$-self dual of Loewy length 3 with socle and cosocle $L$. The term $A$ in the middle is semisimple and the weights of its irreducible summands are given by $L^{u p}$ and the $k$ down moves of $L$ according to section 18.

To determine $\tilde{\mathbb{A}}=D S(\mathbb{A})$ we use induction and lemma 18.5. This implies that $\tilde{\mathbb{A}}$ is the direct sum of $\Pi^{m_{\nu}} \tilde{\mathbb{A}}_{\nu}$ for indecomposable objects $\tilde{\mathbb{A}}_{\nu}$ in $\mathcal{R}_{n-1}$, which uniquely correspond to the irreducible summands of $D S\left(L^{\times 0}\right)$. However these correspond to the irreducible summands $\tilde{L}_{\nu}$ of $D S\left(L^{\text {aux }}\right)$. Again by induction (now induction on the degree of atypicity) the summands of 
$D S\left(L^{\times 0}\right)$ respectively $D S\left(L^{\text {aux }}\right)$ are already known to be given by the derivative of $\lambda_{\text {aux }}$. These facts imply the next

Lemma 20.1. We have

$$
\tilde{\mathbb{A}}=\bigoplus_{\mu=1}^{s} \tilde{\Pi}^{m_{\mu}} \mathbb{A}_{\mu}
$$

where each $\tilde{\mathbb{A}}_{\mu} \in \mathcal{R}_{n-1}$ has Loewy lenght 3 with irreducible socle and cosocle $\tilde{L}_{\mu}$ defined by the $s$ plots for $\mu=1, \ldots, s$

$$
\left[\boxplus_{i} \boxminus_{i+1}\right] \cup S_{\mu}^{\prime} \cup \bigcup_{\nu \neq \mu} S_{\nu} .
$$

In particular, for $m_{\text {aux }}$ (which is congruent to $i+n-1$ modulo 2 ), we get ${ }^{2}$

$$
D S(L) \cong \Pi^{m_{\text {aux }}} L^{\text {aux }} \oplus \bigoplus_{\mu=1}^{s} \tilde{\Pi}^{m_{\mu}} L_{\mu} .
$$

Hence in $K_{0}\left(\mathcal{R}_{n-1}\right)$

$$
d(L)=L^{\prime}=\tilde{L}+(-1)^{i+n-1} \cdot L^{\text {aux }} .
$$

Now each $\tilde{A}_{\mu}$ is determined from $\tilde{L}_{\mu}$ by applying certain upward and the downward moves starting from $\tilde{L}_{\mu}$.

We indicate that the segment of $\tilde{L}_{\mu}$ containing $\left[\boxplus_{i} \boxminus_{i+1}\right]$ has less than $r$ sectors, if $1 \leq \mu \leq j-1$. Indeed the union of the sectors of $\tilde{L}_{\mu}$ in the segment of $\left[\boxplus_{i} \boxminus_{i+1}\right]$ is

$$
\ldots \boxminus S_{\mu+1} \cdots S_{j-1}\left[\boxplus_{i} \boxminus_{i+1}\right] S_{j+1} \cdots S_{r} \boxminus \ldots
$$

for $\mu \leq j-2$ and by $\left[\boxplus_{i} \boxminus_{i+1}\right] S_{j+1} \cdots S_{r} \boxminus \ldots$ for $\mu=j-1$. We are now able to define the detecting objects $A_{\mu}^{\prime} \subseteq \tilde{A}_{\mu}$ for $\mu=1, \ldots, s$ by $\tilde{L}_{\mu}^{\text {down }}$, given by induction as follows

(1) $\left(\int\left(S_{1} \cdots S_{j-1}\right)\right) \boxminus_{i+1} \cup\left(S_{\mu}^{\prime}\right) \cup \bigcup_{j-1<\mu \neq \ell} S_{\ell}$ for $\mu \notin\{1, \ldots, j-1\}$,

(2) $S_{1} \cdots S_{\mu}^{\prime}\left(\int\left(S_{\mu+1} \cdots S_{j-1}\right)\right) \boxminus_{i+1} S_{j+1} \cdots S_{k} \cup \bigcup_{k<\ell} S_{\ell}$ for $\mu \leq j-2$,

(3) $S_{1} \cdots S_{j-2} \boxminus S_{j-1}^{\prime}\left(\boxplus \boxminus_{i}\right) \boxminus_{i+1} S_{j+1} \cdots S_{k} \cup \bigcup_{k<\ell} S_{\ell}$ for $\mu=j-1$.

It is therefore clear that the detecting object is different from all objects in $D S(L)$, which by induction are known to be given by the derivative of $L$. Furthermore $A_{\mu}^{\prime} \subseteq \tilde{A}_{\mu}$. It requires some easy but tedious inspection to see that $A_{\mu}^{\prime}$ is not contained in $\tilde{A}_{\nu}$ for $\nu \neq \mu$. Hence to see that the $A_{\mu}^{\prime}$ are detecting objects, it suffices to show the next

Lemma 20.2. The objects $A_{\mu}^{\prime}$ are contained in $D S(A)$. If $L^{u p}$ is stable, then $L$ is stable and $A_{\mu}^{\prime} \subset H^{+}(A) \oplus H^{-}(A)$ for all $\mu$.

\footnotetext{
${ }^{2}$ assuming that theorem 16.1 holds for $L$, say by induction assumption.
} 
Proof. Recall

$$
A \cong A^{u p} \oplus A^{\text {down }}
$$

for $A^{u p}:=L^{u p}$ and $A^{\text {down }}:=\bigoplus_{i=1}^{k} L_{r}^{\text {down }}$.

We do not know how to compute $D S\left(A^{u p}\right)$. However by induction we already know that the derivative computes $D S\left(A^{\text {down }}\right)$. In $A^{\text {down }} \subset A$ we have the following objects $A_{\mu}$

(1) $\left(\int\left(S_{1} \cdots S_{j-1}\right)\right) \boxminus_{i+1} \cup \bigcup_{j-1<\ell} S_{\ell}$ for $\mu \notin\{1, \ldots, j-1\}$,

(2) $S_{1} \cdots \int\left(S_{\mu}^{\prime} \int\left(S_{\mu+1} \cdots S_{j-1}\right)\right) S_{j+1} \cdots S_{k} \cup \bigcup_{k<\ell} S_{\ell}$ for $\mu \leq j-2$,

(3) $S_{1} \cdots S_{j-2}\left(\boxplus S_{j-1}^{\prime} \boxplus \boxminus_{i} \boxminus_{i+1}\right) S_{j+1} \cdots S_{k} \cup \bigcup_{k<\ell} S_{\ell}$ for $\mu=j-1$.

Their derivative $D S\left(A_{\mu}\right)$ contains

(1) $\left(\int\left(S_{1} \cdots S_{j-1}\right) \boxminus_{i+1}\right) \cup\left(S_{\mu}^{\prime}\right) \cup \bigcup_{j-1<\ell \neq \mu} S_{\ell}$ for $\mu \notin\{1, \ldots, j-1\}$,

(2) $S_{1} \cdots S_{\mu}^{\prime}\left(\int\left(S_{\mu+1} \cdots S_{j-1}\right)\right) \boxminus_{i+1} S_{j+1} \cdots S_{k} \cup \bigcup_{k<\ell} S_{\ell}$ for $\mu \leq j-2$,

(3) $S_{1} \cdots S_{j-2} \boxminus S_{j-1}^{\prime}\left(\boxplus \boxminus_{i}\right) \boxminus_{i+1} S_{j+1} \cdots S_{k} \cup \bigcup_{k<\ell} S_{\ell}$ for $\mu=j-1$.

This proves $A_{\mu}^{\prime} \subset D S\left(A_{\mu}\right)$ and hence our claim.

Commutation rule for algorithm I. Now we discuss how moves commute with differentiation for a given $L$ as above. It is rather obvious from the definitions that for this we can restrict ourselves to the situation where $L$ is the single segment

$$
L=\left(S_{1} \cdots S_{j-1} \boxplus_{i} \boxminus_{i+1} S_{j+1} \cdots S_{k}\right) .
$$

So let us assume this for simplicity of exposition.

1) Computation of $\tilde{A}$. Taking first the derivative we obtain $L^{\text {aux }}$ and $(k-1)$ plots $\tilde{L}_{\mu}$ of the form

$$
S_{1} \cdots S_{\mu}^{\prime}\left(S_{\mu+1} \cdots S_{j-1} \boxplus_{i} \boxminus_{i+1} S_{j+1} \cdots S_{k}\right)
$$

(lower group where $\mu \leq j-1$ ) respectively

$$
\left(S_{1} \cdots S_{j-1} \boxplus_{i} \boxminus_{i+1} S_{j+1} \cdots S_{\mu-1}\right) S_{\mu}^{\prime} \cdots S_{k}
$$

(upper group where $\mu \geq j+1$ ). The sign in the Grothendieck group attached to these is $(-1)^{a_{\mu}+n-1}=(-1)^{i+n-1}$ for $S_{\mu}=\left[a_{\mu}, b_{\mu}\right]$.

Notice that $L^{a u x}$ does not define any moves. The segment containing $\boxplus_{i} \boxminus_{i+1}$ (indicated by the brackets) defines the possible moves of each of these derived plots $\tilde{L}_{\mu}$. These are e.g. in the lower group case the upward move

$$
S_{1} \cdots S_{\mu}^{\prime} S_{\mu+1} \cdots S_{j-1} \boxminus_{i} \int\left(S_{j+1} \cdots S_{k}\right)
$$

and the downward move

$$
S_{1} \cdots S_{\mu}^{\prime} \int\left(S_{\mu+1} \cdots S_{j-1}\right) \boxminus_{i+1} S_{j+1} \cdots S_{k}
$$


and the internal upper/lower downward moves

$$
\begin{aligned}
& S_{1} \cdots S_{\mu}^{\prime} S_{\mu+1} \cdots S_{j-1} \int\left(\int\left(S_{j+1} \cdots S_{r-1}\right) S_{r}^{\prime}\right) S_{r+1} \cdots S_{k} \\
& S_{1} \cdots S_{\mu}^{\prime} S_{\mu+1} \cdots S_{r-1} \int\left(S_{r}^{\prime} \int\left(S_{r+1} \cdots S_{j-1}\right)\right) S_{j+1} \cdots S_{k}
\end{aligned}
$$

2) Computation of the derivative $A^{\prime}$. Now we revert the situation and first consider the moves of $L$, the upward and downward moves

$$
\begin{aligned}
L^{u p} & =S_{1} \cdots S_{j-1} \boxminus_{i} \int\left(S_{j+1} \cdots S_{k}\right), \\
L^{\text {down }} & =\int\left(S_{1} \cdots S_{j-1}\right) \boxminus_{i+1} S_{j+1} \cdots S_{k},
\end{aligned}
$$

and the internal downward moves (for lower sectors)

$$
S_{1} \cdots S_{r-1} \int\left(S_{r}^{\prime} \int\left(S_{r+1} \cdots S_{j-1}\right)\right) S_{j+1} \cdots S_{k}
$$

respectively (for upper sectors)

$$
S_{1} \cdots S_{j-1} \int\left(\int\left(S_{j+1} \cdots S_{r-1}\right) S_{r}^{\prime}\right) S_{r+1} \cdots S_{k} .
$$

If we differentiate $L^{u p}$, we get the plots of the form

$$
S_{1} \cdots S_{\mu}^{\prime} \cdot S_{j-1} \boxminus_{i} \int\left(S_{j+1} \cdots S_{k}\right)
$$

with sign $(-1)^{s_{\mu}+n-1}=(-1)^{i+n-1}$ and similarly

$$
L^{a u x}=S_{1} \cdots S_{j-1} \boxminus_{i} \boxminus_{i+1} S_{j+1} \cdots S_{k}
$$

with sign $(-1)^{i+n}$. If we differentiate $L^{\text {down }}$, we get $\int\left(S_{1} \cdots S_{j-1}\right) \boxminus_{i+1}$ $S_{j+1} \cdots S_{\mu}^{\prime} \cdots S_{k}$ and similarly

$$
L^{a u x}=S_{1} \cdots S_{j-1} \boxminus_{i} \boxminus_{i+1} S_{j+1} \cdots S_{k}
$$

with sign $(-1)^{i+n}$. If we derive the plots defined by the internal moves (lower group, where we derive at $\nu \leq j-1$ ) we get the plots of the form

$$
S_{1} \cdots S_{r-1} S_{r}^{\prime} \int\left(S_{r+1} \cdots S_{j-1}\right) \boxminus_{i+1} S_{j+1} \cdots S_{k}
$$

with sign $(-1)^{s_{r}+n-1}=(-1)^{i+n-1}$ together with

$$
\begin{aligned}
& S_{1} \cdots S_{\mu}^{\prime} S_{\mu+1} \cdots S_{j-1} \int\left(\int\left(S_{j+1} \cdots S_{r-1}\right) S_{r}^{\prime}\right) S_{r+1} \cdots S_{k} \\
& S_{1} \cdots S_{\mu}^{\prime} S_{\mu+1} \cdots S_{r-1} \int\left(S_{r}^{\prime} \int\left(S_{r+1} \cdots S_{j-1}\right)\right) S_{j+1} \cdots S_{k}
\end{aligned}
$$

of sign $(-1)^{i+n-1}$ respectively similar terms for the upper group, where we differentiate at $\mu \geq j+1$. Altogether, besides two additional signed plots of the form $L^{\text {aux }}$, these give precisely the plots obtained before. This implies 
Lemma 20.3. The differential of the moves of $L$ gives the term $2 \cdot L^{\text {aux }}$ plus the moves of the differential of L, i.e.

$$
A^{\prime}=\tilde{A}+2(-1)^{i+n} \cdot L^{\text {aux }}
$$

holds in $K_{0}\left(\mathcal{R}_{n-1}\right)$.

Algorithm II (melting sectors). Suppose $\lambda_{\wedge \vee}$ is a plot with a single segment and at least two sectors. $[a, i]$ and point $i$ respectively left boundary point $i+1$ of the segment defining the plot $\lambda_{\wedge \vee}$. In algorithm II we melt the first two adjacent sectors $[a, i]$ and $[i+1, b]$ together into a single sector $S^{\text {melt }}$ to obtain a new plot $\lambda_{\vee \wedge}$ so that

$$
\operatorname{supp}\left(\lambda_{\wedge \vee}\right)-\{i+1\}=\operatorname{supp}\left(\lambda_{\vee \wedge}\right)-\{i\} .
$$

This new plot $\lambda_{\wedge \vee}$ again has a unique segment with the same underlying interval as the plot $\lambda_{\wedge \vee}$. But the sector structure is different, since the number of sectors decreases by one.

Notice, opposed to algorithm I, the interval $[i, i+1]$ does not define a sector of the original plot $\lambda_{\wedge \vee}$. However $[i, i+1]$ defines a sector of the 'internal' plot

$$
L_{i n t}:=\partial\left(S^{m e l t}\right)
$$

with sector structure say

$$
L_{i n t}=S_{1} \cdots S_{j-1}\left[\boxplus_{i} \boxminus_{i+1}\right] S_{j+1} \cdots S_{k},
$$

so that

$$
\lambda_{\vee \wedge}=\left(\int L_{i n t}\right) \text { other sectors } \quad, \quad \lambda_{\times 0}:=\left(\int\left(L_{i n t}\right)^{\times 0}\right) \text { other sectors }
$$

We similarly define for $r=1, \ldots, k$ and $r \neq j$ the plots

$$
\lambda_{r}^{\text {down }}:=\left(\int\left(L_{\text {int }}\right)_{r}^{\text {down }}\right) \text { other sectors } .
$$

Finally $\lambda_{\wedge \vee}=\left(\int\left(L_{i n t}\right)^{u p}\right)$ other sectors, which is the plot we started from. Since $\int\left(L_{\text {int }}\right)^{u p}$ has two sectors, all the plots $\lambda_{r}^{\text {down }}$ for $1 \leq r \neq j \leq k$ have less sectors than the plot $\lambda_{\wedge \vee}$. Indeed, the plots $\int\left(L_{i n t}\right)_{r}^{d o w n}$ are irreducible as an easy consequence of the integral criterion.

Construction of detecting objects for algorithm II. Fixing $\lambda_{\wedge \vee}$ as above,

$$
\mathbb{A}=F_{i}\left(L\left(\lambda_{\times 0}\right)\right)=(L, A, L)
$$

defines a $*$-self dual object in $\mathcal{R}_{n}$ of Loewy length 3 with socle and cosocle $L$, where

$$
L=L\left(\lambda_{\vee \wedge}\right)
$$

and $A=A^{u p} \oplus A^{\text {down }}$ for $A^{u p}=L\left(\lambda_{\wedge \vee}\right)$ and $A^{\text {down }}=\bigoplus_{r \neq j, 1}^{k} L\left(\lambda_{r}^{\text {down }}\right)$. 
To determine $D S(\mathbb{A})=\bigoplus_{\mu} \Pi^{m_{\mu}} \tilde{\mathbb{A}}_{\mu}$ we use induction and lemma 18.5. This implies that $\tilde{\mathbb{A}}$ is the direct sum of $\tilde{\Pi}^{m_{\mu}} \mathbb{A}_{\mu}$ for indecomposable objects $\tilde{\mathbb{A}}_{\mu}$ in $\mathcal{R}_{n-1}$, which uniquely correspond to the irreducible summands $\tilde{L}_{\mu}$ of $D S\left(L^{\times \circ}\right)$. But by induction (now induction on the degree of atypicity !) the irreducible summands of $D S\left(L^{\times 0}\right)$, that determine the irreducible modules $\tilde{L}_{\mu}$, can be computed by the derivative of $\lambda_{\text {aux }}$. Since in the present situation replacing $i, i+1$ by $\times, \circ$ commutes with the derivative, these facts imply the next

Lemma 20.4. If L has s sectors, for the melting algorithm we have

$$
\tilde{\mathbb{A}}=\bigoplus_{\mu=1}^{s} \Pi^{m_{\mu}} \tilde{\mathbb{A}}_{\mu}
$$

where each $\tilde{\mathbb{A}}_{\mu} \in \mathcal{R}_{n-1}$ has Loewy length 3 of Loewy structure $\left(\tilde{L}_{\mu}, \tilde{A}_{\mu}, \tilde{L}_{\mu}\right)$ with irreducible socle and cosocle $\tilde{L}_{\mu}$. For the various summands, for varying $\mu$, up to the shift $m_{\mu}$ the socles $L_{\mu}$ are defined by the $s-1$ different plots plots arising from the derivative

$$
\text { ( } \left.\left.\int L_{\text {int }}\right) \text { (other sectors }\right)^{\prime}
$$

together with the plot

$$
L_{\text {int }} \text { (other sectors) . }
$$

In particular ${ }^{3}$, if $L$ has $s$ sectors, $D S(L) \cong \bigoplus_{\mu=1}^{s} \Pi^{m_{\mu}} \tilde{L}_{\mu}$. This gives in $K_{0}\left(\mathcal{R}_{n-1}\right)$ the formula

$$
d(L)=L^{\prime}=\tilde{L} \text {. }
$$

Corollary 20.5. In the situation of the last lemma the morphisms

$$
H^{i}(p): H^{i}(\mathbb{A}) \rightarrow H^{i}(L)
$$

are surjective for all $i \in \mathbb{Z}$.

Proof. We already know that $H^{ \pm}(p): H^{ \pm}(\mathbb{A}) \rightarrow H^{ \pm}(L)$ induces injective maps on the cosocle of $H^{ \pm}(\mathbb{A})$. By lemma 20.4 therefore these induced maps are bijections between the cosocle of $H^{ \pm}(\mathbb{A})$ and $H^{ \pm}(L)$. In particular the morphisms $H^{ \pm}(p): H^{ \pm}(\mathbb{A}) \rightarrow H^{ \pm}(L)$ are surjective. This implies the assertion.

This being said note that $2 d(L)+d(A)=d(\mathbb{A})=d(\tilde{\mathbb{A}})=2 \tilde{L}+\tilde{A}$ together with the assertion $d(L)=\tilde{L}$ from the lemma 20.4 above implies $d(A)=$ $\tilde{A}$. Any $\tilde{L}_{\mu}$ defines a nontrivial term $\tilde{A}_{\mu}$. We claim that any irreducible summand $A_{\mu}^{\prime} \subset \tilde{A}_{\mu}$ is a detecting object now. Indeed any summand $A_{\mu}^{\prime}$ of $\tilde{A}$ appears in $H^{+}(A)$ by the formula $d(A)=H^{+}(A)-H^{-}(A)=\tilde{A}$. Checking

\footnotetext{
${ }^{3}$ assuming that theorem 16.1 holds for $L$ and $L^{\times \circ}$, say by induction assumption.
} 
the possible moves that define the constituents of $\tilde{A}_{\mu}$ from $\tilde{L}_{\mu}$ it is clear that $A_{\mu}^{\prime}$ is not a constituent of any $\tilde{A}_{\nu}$ for $\nu \neq \mu$. Hence

Lemma 20.6. Detecting objects $A_{\nu}^{\prime}$ exist for algorithm II.

Commutation rule for algorithm II. Now we discuss how moves commute with differentiation for a given $L$ as above. It is rather obvious from the definitions that we can restrict ourselves for this to the situation where the segment of plot $\lambda_{\wedge \vee}$ has only two sectors. In other words we claim that we can assume without restriction of generality that the terms 'other factors' does not appear, so that $s=2$ holds in the last lemma 20.4. The reason for this is, that moves for $L_{i n t}$ (others sectors) are the same as for $\int L_{\text {int }}$ (others sectors)', since by [BS10a] the relevant moves are moves 'within' the sector $\int L_{\text {int }}$. Hence for the proof of the next lemma we can assume that $L=\int L_{\text {int }}$ has a unique sector so that $d(L)=\tilde{L}=L^{\prime}=L_{\text {int }}$, which has a single segment.

Lemma 20.7. The differential of the moves of $L$ gives the moves of the differential of $L$, i.e.

$$
A^{\prime}=\tilde{A}
$$

holds in $K_{0}\left(\mathcal{R}_{n-1}\right)$.

Proof. Without restriction of generality we can assume that the plot $\lambda_{\vee \wedge}$, we are starting with, is a segment with only two sectors, so that $L=\int L_{\text {int }}$. Let the single segment of $L_{i n t}$ have the form

$$
S_{1} \cdots S_{j-1}\left[\boxplus_{i} \boxminus_{i+1}\right] S_{j+1} \cdots S_{k}
$$

with $k$ sectors $S_{1}, \ldots, S_{k}$ where the underlying interval of $S_{j}$ is $[i, i+1]$.

1) Computation of $\tilde{A}$. According to [BS10a], [Wei10] the constituents of $\tilde{\mathbb{A}}=\bigoplus_{\mu=1}^{s} \Pi^{m_{\mu}} \tilde{\mathbb{A}}_{\mu}$ are obtained from the socle module $\tilde{L}_{\mu}$ of $\tilde{\mathbb{A}}_{\mu}$ by moves. The last lemma shows that $\tilde{L}=\bigoplus_{\mu} \tilde{L}_{\mu}$ is the derivative $L^{\prime}=\left(\int L_{\text {int }}\right)^{\prime}=L_{\text {int }}$ of $L$ up to a shift determined by the sign factor $(-1)^{a+n-1}$. Since $s=1$ by assumption, $\tilde{\mathbb{A}}=(\tilde{L}, \tilde{A}, \tilde{L})$ is an indecomposable module with socle

$$
\tilde{L}=L_{\text {int }} \text {. }
$$

Up to a parity shift by $m=a+n-1$, the module $\tilde{A}$ therefore is the direct sum

$$
\tilde{A}=\left(L_{i n t}\right)^{u p} \oplus \bigoplus_{r=1}^{k}\left(L_{i n t}\right)_{r}^{d o w n}
$$


of the irreducible modules obtained from $\tilde{L}=L_{\text {int }}$ by the unique upward and the $k$ downward moves. Notice $\left(L_{i n t}\right)_{j}^{\text {down }}=\left(L_{i n t}\right)^{\text {down }}$ is the 'nonencapsulated' downward move in the notions of [Wei10]. Here it occurs, since $[i, i+1]$ is one of the sectors of the $\tilde{L}$.

2) Computation of the derivative $A^{\prime}$. Now we revert the situation and first consider $\mathbb{A}=(L, A, L)$ and the moves of $L=\int L_{\text {int }}$ that determine the irreducible summands of $A$. Indeed

$$
A=\left(\int L_{\text {int }}\right)^{u p} \oplus \bigoplus_{r \neq j, r=1}^{k}\left(\int L_{\text {int }}\right)_{r}^{\text {down }}
$$

holds for the irreducible modules obtained from $L=\int L_{\text {int }}$ by the upward move $L^{u p}$ and the $k-1$ internal inner/upper downward moves $\left(L_{\text {int }}\right)_{r}^{\text {down }}$ for $r \neq j$. Notice that $\left(L_{i n t}\right)_{j}^{\text {down }}=\left(L_{\text {int }}\right)^{\text {down }}$, as opposed to the situation above, this time does not appear as a move, since we are in the 'encapsulated' case in the notions [Wei10] where $[i, i+1]$ is not a sector of $L$ (but only an internal sector of $L$ ).

The formulas above imply that $A^{\prime}=\left(A^{\text {up }}\right)^{\prime} \oplus\left(A^{\text {down }}\right)^{\prime}$ is a direct sum of the two irreducible summands

$$
\left(A^{u p}\right)^{\prime}=B_{1} \oplus B_{2},
$$

coming from $\left(\left(\int L_{\text {int }}\right)^{u p}\right)^{\prime}=\left(L^{u p}\right)^{\prime}=L\left(\lambda_{\wedge \vee}\right)^{\prime}$ for $\lambda_{\wedge \vee}=[a, i][i+1, b]$ with derivative $(-1)^{a+n-1}(\partial([a, i]) \cup[i+1, b])+(-1)^{i+n-1}([a, i] \cup \partial([i+1, b]))$, and the $k-1$ irreducible summands $\left(A_{r}^{\text {down }}\right)^{\prime}$ of $\left(A^{\text {down }}\right)^{\prime}$ given by

$$
\left(A_{r}^{\text {down }}\right)^{\prime}=\left(\left(\int L_{\text {int }}\right)_{r}^{\text {down }}\right)^{\prime} \text {. }
$$

This gives $2+(k-1)=k+1$ irreducible factors in $\tilde{A}$, and all signs coincide by $(-1)^{i+n-1}=(-1)^{a+n-1}$.

The comparison. Since all signs are $(-1)^{a+n-1}$ for both computations, we can ignore the parity shift. Then observe that $\left.\left(\int L_{\text {int }}\right)_{r}^{\text {down }}=\int\left(L_{\text {int }}\right)_{r}^{\text {down }}\right)$ holds for $r \neq j$, hence $\left.\left(A_{r}^{\text {down }}\right)^{\prime}=\left(\left(\int L_{\text {int }}\right)_{r}^{\text {down }}\right)^{\prime}=L_{\text {int }}\right)_{r}^{\text {down }}$ for $r \neq j$. So it remains to compare the two remaining summands

$$
B_{1} \quad, \quad B_{2}
$$

of $A^{\prime}$ and the two remaining summands

$$
\left(L_{\text {int }}\right)^{u p} \quad, \quad\left(L_{i n t}\right)_{j}^{\text {down }}
$$

of $\tilde{A}$. The latter correspond to the plots $S_{1} \ldots S_{j-1} \boxminus_{i} \int\left(S_{j+1} \ldots S_{k}\right)$, giving the upward move, resp. $\int\left(S_{1} \ldots S_{j-1}\right) \boxminus_{i+1} S_{j+1} \cdots S_{k}$, giving the downward move. Obviously these two define the plots $\partial([a, i]) \cup[i+1, b]$ respectively $[a, i] \cup \partial([i+1, b])$ defining the two summands $B_{1}$ and $B_{2}$. 


\section{Part 3. Consequences of the Main TheOrem}

We describe some applications of the main theorem. The main result is the computation of the $\mathbf{Z}$-grading of $D S(L)$ for any irreducible representation $L$ in sections 22 - 24. This result is based on the main theorem and its proof. For that we first need a description of the dual of an irreducible representation in section 21 . In the later sections 28 - 31 we obtain various results about the cohomology of maximally atypical indecomposable representations.

\section{TANnaka Duals}

Let $\lambda$ be an atypical weight, and $L(\lambda)$ the associated irreducible representation. Note that $\left(B e r^{k} \otimes L(\lambda)\right)^{\vee}=B e r^{-k} \otimes L(\lambda)^{\vee}$. We use the description of the duals obtained in [Hei14]. Note that $L(\lambda)=\operatorname{socle}(P(\lambda))=$ cosocle $(P(\lambda))$, since projective modules are $*$-self dual. Hence $L(\lambda)^{\vee}=$ socle $\left(P(\lambda)^{\vee}\right)$, so it suffices to compute the socle of $P(\lambda)^{\vee}$. Now $P(\lambda)=$ $R\left(\lambda^{L}, \lambda^{R}\right)$ for the bipartition $\left(\lambda^{L}, \lambda^{R}\right)=\theta^{-1}(\lambda)$ satisfying $k\left(\lambda^{L} ; \lambda^{R}\right)=n$ by [Hei14]. The dual of any mixed tensor is $R\left(\lambda^{L}, \lambda^{R}\right)^{\vee}=R\left(\lambda^{R}, \lambda^{L}\right)$, hence we simply have to calculate the socle of $R\left(\lambda^{R}, \lambda^{L}\right)$.

If $k\left(\lambda^{L}, \lambda^{R}\right)=n$, the description of the map $\theta$ is easy: Calculate the weight diagram of $\left(\lambda^{L}, \lambda^{R}\right)$ as in section 14 and write down its labeled cup diagram. Then turn all $\vee$ 's which are not part of a cup into $\wedge$ 's and leave all other symbols unchanged. The resulting diagram is the weight diagram of socle $(P(\lambda))$. Hence in order to calculate the dual of $L(\lambda)$ we simply have to understand the effect of changing $\left(\lambda^{L}, \lambda^{R}\right)$ to $\left(\lambda^{R}, \lambda^{L}\right)$ on the weight diagram. Recall from section 14 that

$$
I_{\wedge}(\lambda):=\left\{\lambda_{1}^{L}, \lambda_{2}^{L}-1, \lambda_{3}^{L}-2, \ldots\right\} \quad \text { and } \quad I_{\vee}(\lambda):=\left\{1-\lambda_{1}^{R}, 2-\lambda_{2}^{R}, \ldots\right\} .
$$

If $\lambda_{i}^{L}-(i-1)=s$, then $i-\lambda_{i}^{L}=i-s-i+1=1-s$ and likewise for $\lambda_{j}^{R}$. Hence interchanging $\lambda^{L}$ and $\lambda^{R}$ means reflecting the symbols $s \mapsto 1-s$ and swapping $\vee$ 's with $\wedge$ 's. If the vertex $s$ is labelled by a $\times$, then there exist $i, j$ such that $\lambda_{i}^{L}-(i-1)=j-\lambda_{j}^{R}=s$. But then $\lambda_{j}^{R}-(j-1)=i-\lambda_{i}^{L}=1-s$ and we obtain a $\times$ at the vertex $1-s$. We argue in the same way for the $o$ 's. If $(s, s+r)$ is labelled by $(\vee, \wedge)$-pair such that we have a cup connecting $s$ and $s+r$, we obtain a $(\vee, \wedge)$-pair at $(1-s-r, 1-s)$ which is connected by a cup. To obtain the highest weight $\theta\left(\lambda^{R}, \lambda^{L}\right)$ the $\vee$ 's not in cups get flipped to $\wedge$ 's. 
Proposition 21.1. The weight diagram of the dual of an irreducible representation $L$ is obtained from the weight diagram of $L$ as follows: Interchange all $\vee \wedge$-pairs in cups, then apply the reflection $s \mapsto 1-s$ to each symbol.

It is easy to see that this description is valid for $m \geq n$ if we use the reflection $1-\delta-s$ instead of $1-s$ where $\delta=m-n$.

The maximal atypical case. We describe the dual in the language of plots. We assume here that $L(\lambda)$ is maximally atypical, but we can reduce the general case to this one, using the map $\phi$ from section 18 and lemma 21.3. Let $\lambda$ denote the unique plot corresponding to the weight $\lambda$. Let $\lambda(s)=$ $\prod_{i} \lambda_{i}(s)$ be its prime factorization. For each prime factor $\lambda_{i}(s)=(I, K)$ with segment $I$ and support $K$ we define $\lambda_{i}^{c}(s):=\left(I, K^{c}\right)$, where $K^{c}=I-K$ denotes the complement of $K$ in $I$. Then put

$$
\lambda^{c}(s):=\prod_{i} \lambda_{i}^{c}(s) .
$$

The previous description of the duals implies the next proposition.

Proposition 21.2. The Tannaka dual representation $\lambda^{\vee}$ of a maximal atypical representation $\lambda$ is given by the plot

$$
\lambda^{\vee}(s)=\lambda^{c}(1-s) .
$$

Example 1. Suppose $\lambda=\left[0, \lambda_{2}, \ldots, \lambda_{n}\right]$ holds with $0>\lambda_{2}$ and $\lambda_{i}>\lambda_{i+1}$ for $2 \leq i \leq n-1$. Then $\lambda^{\vee}=\left[n-\lambda_{n}-1, n-\lambda_{n-1}-1, \ldots, n-\lambda_{2}-1, n-1\right]$.

Dualising is compatible with the normalized block equivalence $\phi_{n}^{i}$ of section 15.

Lemma 21.3. For irreducible $i$-atypical $L$ we have $\phi_{n}^{i}\left(L^{\vee}\right)=\phi_{n}^{i}(L)^{\vee}$.

Proof. If $L$ is $i$-atypical, then $\tilde{\phi}_{n}^{i}$ preserves the distances between the sectors, hence $\tilde{\phi}_{n}^{i}(L)^{\vee}=\operatorname{Ber} \cdots \otimes \tilde{\phi}_{n}^{i}\left(L^{\vee}\right)$. Since we remove $2(n-i)$ symbols from the weight diagram of $L$, we obtain the shift

$$
\tilde{\phi}_{n}^{i}\left(L^{\vee}\right)=\operatorname{Ber}^{-2(n-i)} \otimes \tilde{\phi}_{n}^{i}(L)^{\vee} .
$$

Now we calculate for the normalised block equivalence

$$
\begin{aligned}
\phi_{n}^{i}\left(L^{\vee}\right) & =\operatorname{Ber}^{n-k} \tilde{\phi}_{n}^{i}\left(L^{\vee}\right)=\operatorname{Ber}^{n-k} \operatorname{Ber}^{-2(n-k)} \tilde{\phi}_{n}^{i}(L)^{\vee} \\
& =B e r^{-n+k} \otimes \tilde{\phi}_{n}^{i}(L)^{\vee}=\left(\operatorname{Ber}^{n-k}\right)^{\vee} \otimes \phi(L)^{\vee}=\left(\operatorname{Ber}^{n-k} \otimes \tilde{\phi}_{n}^{i}(L)\right)^{\vee} \\
& =\phi_{n}^{i}(L)^{\vee} .
\end{aligned}
$$

Lemma 21.4. For maximal atypical irreducible $L=\left[\lambda_{1}, \ldots, \lambda_{n}\right]$ such that $\lambda_{n}=0$ the following assertions are equivalent. 
(1) $L^{\vee} \cong\left[\rho_{1}, \ldots, \rho_{n}\right]$ holds such that $\rho_{n} \geq 0$.

(2) L is basic, i.e. $\lambda_{1} \geq \ldots \lambda_{n} \geq 0$ and $\lambda_{i} \leq n-i$ holds for all $i=1, \ldots, n$.

(3) $\lambda_{1} \leq n-1$ and $L^{\vee} \cong\left[\lambda_{1}^{*}, \ldots, \lambda_{n}^{*}\right]$ holds for the transposed partition $\lambda^{*}=\left(\lambda_{1}^{*}, \ldots, \lambda_{n}^{*}\right)$ of the partition $\lambda=\left(\lambda_{1}, \ldots, \lambda_{n}\right)$.

Remark. The number of basic maximal atypical weights in $X^{+}(n)$ is equal to the Catalan number $C_{n}$.

Proof. i) implies ii): If $\rho_{n}=0$ the leftmost $\vee$ in the weight diagram of $[\rho]$ is at position $-n+1$. Then the smallest $\wedge$ bound in a cup is at a position $\leq 1$ and $\geq 1-n$. After the change $(I, K) \rightarrow(I, I-K)$ and the reflection $s \mapsto 1-s$ this means that the rightmost $\vee$ in $[\rho]^{\vee}$ is at position $\leq n-1$ and $\geq 0$ which is equivalent to $0 \leq \lambda_{1} \leq n-1$. Likewise the $i$-th leftmost $\wedge$ bound in a cup is at a position $\geq-n+i+1$ and $\leq n$. It will give the $i$-th largest $\vee$ in the weight diagram of $[\lambda]$. After the change $(I, K) \mapsto(I, I-K)$ and the reflection the $i$-th largest $\vee$ is at a position $\leq n-2 i+1$ which is equivalent to $\lambda_{i} \leq n-i$. ii) implies $\mathrm{i}$ ): If $\lambda$ is basic the largest $\vee$ is at position $\leq n-1$, hence the largest $\wedge$ bound in a cup is at position $\leq n$. It gives the smallest $\vee$ of $[\lambda]^{\vee}$. Hence the smallest $\vee$ of $[\lambda]^{\vee}$ is at a position $\geq 1-n$ which is equivalent to $\lambda_{n}^{\vee} \geq 0$.

ii) implies iii): If $\lambda$ is basic, the $2 n$ vertices in cups form the intervall $J:=[-n+1, n]$ of length $2 n$. If $J_{\vee}$ is the subset of vertices labelled by $\vee$, the subset $J \backslash J_{\vee}$ is the subset of vertices labelled by $\wedge$. The intervall $J$ is preserved by the reflection $s \mapsto 1-s$. If $\lambda$ is basic, so is $\lambda^{*}$. We use the following notation: If

$$
\lambda_{1}=\ldots=\lambda_{s_{1}}>\lambda_{s_{1}+1}=\ldots=\lambda_{s_{2}}>\lambda_{s_{2}+1}=\ldots=\lambda_{s_{r}}>\lambda_{s_{r}+1}=0
$$

put $\delta_{1}=s_{1}$ and $\delta_{i}=s_{i}-s_{i-1}$ and $\Delta_{i}=\lambda_{s_{i}}-\lambda_{s_{i}+1}$ : Likewise for $\lambda^{*}$ with $\delta_{i}^{*}$ and $\Delta_{i}^{*}$. Then

$$
\delta_{i}=\Delta_{i-r}^{*}, \Delta_{i}=\delta_{i-r}^{*} .
$$

Then the weight diagram of $\left[\lambda^{*}\right]$ looks, starting from $n$ and going to the left

$$
\ldots \nabla \overbrace{\ldots \nabla}^{\delta_{3}^{*}} \overbrace{\wedge \ldots \wedge}^{\Delta_{2}^{*}} \overbrace{\nabla \ldots \nabla}^{\delta_{2}^{*}} \overbrace{\wedge \ldots \wedge}^{\Delta_{1}^{*}} \wedge \overbrace{}^{\delta_{1}^{*}} \wedge \nabla \wedge \wedge
$$

and the weight diagram of $[\lambda]$ looks, starting from $-n+1$ and going to the right like

$$
\vee \ldots \vee \overbrace{\wedge \ldots \wedge}^{\Delta_{r}=\delta_{1}^{*}} \overbrace{\nabla \ldots \vee}^{\delta_{r}=\Delta_{1}^{*}} \overbrace{\overbrace{\ldots . \wedge}}^{\Delta_{r-1}=\delta_{2}^{*}} \overbrace{\nabla \ldots \vee}^{\delta_{r-1}=\Delta_{2}^{*}} \ldots
$$

The two weight diagrams are mirror images of each other and the rule for the $\vee$ 's in cups in one is the same as the rule for the $\wedge$ 's in the cups of the other. Hence after the change $(I, K) \mapsto(I, I-K)$ and the reflection $s \mapsto 1-s$ the two weight diagrams agree. iii) implies i): trivial. 
Example 3. Duals in the $\mathcal{R}_{3}$-case. If $a>b>0$, then $[a, b, 0]^{\vee}=[2,2-$ $b, 2-a]=\operatorname{Ber}^{2-a}[a, a-b, 0]$. If $a \geq 1$ then $[a, a, 0]^{\vee}=[2,1-a, 1-a]=$ $\operatorname{Ber}^{1-a}[a+1,0,0]=\operatorname{Ber}^{1-a} S^{a+1}$.

A better description. If $L=L(\lambda)$ is an irreducible maximal atypical representation in $\mathcal{R}_{n}$, its weight $\lambda$ is uniquely determined by its plot. Let $S_{1} \ldots S_{2} \ldots S_{k}$ denote the segments of this plot. Each segment $S_{\nu}$ has even cardinality $2 r\left(S_{\nu}\right)$, and can be identified up to a translation with a unique basic weight of rank $r\left(S_{\nu}\right)=r_{\mu}$ and a partition in the sense of lemma 21.4. For the rest of this section we denote the segment of rank $r\left(S_{\nu}\right)$ attached to the dual partition by $S_{\nu}^{*}$, hoping that this will not be confused with the contravariant functor $*$. Using this notation, Tannaka duality maps the plot $S_{1} \ldots S_{2} \ldots S_{k}$ to the plot $S_{k}^{*} \ldots S_{2}^{*} . . S_{1}^{*}$ so that the distances $d_{i}$ between $S_{i}$ and $S_{i+1}$ coincide with the distances between $S_{i+1}^{*}$ and $S_{i}^{*}$. This follows from proposition 21.2 and determines the Tannaka dual $L^{\vee}$ of $L$ up to a Berezin twist.

The dual forest. If we identify the basic plots with rooted trees $S_{i} \leftrightarrow \mathcal{T}_{i}$, we can describe a weight by a spaced forest

$$
\mathcal{F}=\left(d_{0}, \mathcal{T}_{1}, d_{1}, \mathcal{T}_{2}, \ldots, d_{k-1}, \mathcal{T}_{k}\right)
$$

where $d_{0}=\lambda_{n}$. We describe the dual in this language.

Grafting. Given a planar forest $\mathcal{F}=\mathcal{T}_{1} \ldots \mathcal{T}_{n}$ of planar rooted trees, we can introduce a new $n$-ary root and graft the trees $\mathcal{T}_{i}$ onto this root. This new tree is called the grafting product $\vee\left(\mathcal{T}_{1} \ldots \mathcal{T}_{n}\right)$ of $\mathcal{T}_{1} \ldots \mathcal{T}_{n}$. The grafting product of the trees in a spaced forest is obtained by forgetting the distances and simply taking the grafting product of the trees.

Example. Consider the forest of two rooted planar trees

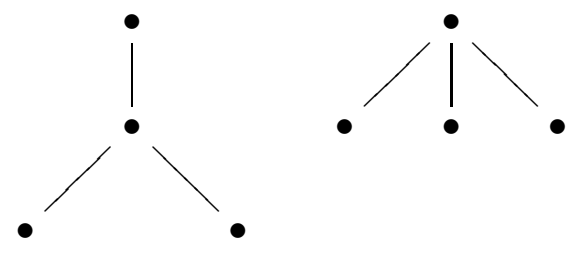

Grafting this planar forest gives the forest with the single tree 


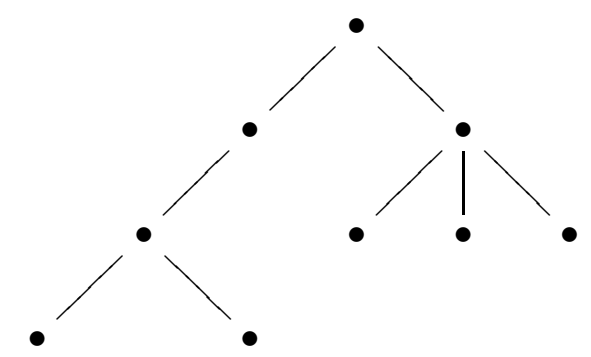

Mirror tree. If $\mathcal{T}$ is a planar rooted tree, then the mirror image $\mathcal{T}^{*}$ of $\mathcal{T}$ along the root axis is recursively defined as follows: Put $(\vee(\emptyset))^{*}=$ $\vee(\emptyset), \emptyset^{*}=\emptyset$ where $\emptyset$ is the empty tree and extend via

$$
\left(\vee\left(\mathcal{T}_{1} \ldots \mathcal{T}_{n}\right)\right)^{*}=\vee\left(\mathcal{T}_{n}^{*} \ldots \mathcal{T}_{1}^{*}\right)
$$

Example. The mirror image of the grafted planar tree above is

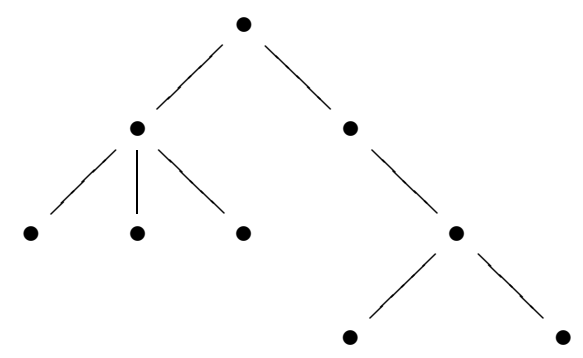

Lemma 21.5. The weight of the dual representation corresponds to the spaced forest

$$
\mathcal{F}^{\vee}=\left(d_{0}^{*}, \mathcal{T}_{k}^{*}, d_{1}^{*}, \mathcal{T}_{k-1}^{*}, d_{2}^{*}, \ldots, d_{k-1}^{*}, \mathcal{T}_{1}^{*}\right)
$$

where $d_{i}^{*}:=d_{k-i}$ for $i=1, \ldots, k-1$ and $d_{0}^{*}=-d_{0}-d_{1}-\ldots-d_{k-1}$ and $\mathcal{T}_{i}^{*}$ denotes the mirror image (along the root axis) of the planar tree $\mathcal{T}_{i}$.

Proof. The claim about the distances $d_{1}^{*}, \ldots, d_{k-1}^{*}$ follows from the description of the dual plots. We first prove the claim about $d_{0}^{*}$. Now $d_{0}^{*}=$ $(1-b)+n-1$ where $b$ is the last point of the rightmost sector with rank $r_{k}$

$$
b=\lambda_{1}+\left(2 r_{k}-1\right) \text {. }
$$

Hence $d_{0}^{*}=-b+n=-\lambda_{1}-2 r_{k}+n+1$. Now use that $\lambda_{1}=\left(\lambda_{n}-n+1\right)+$ $2 r_{1}+\ldots+2 r_{k-1}+d_{1}+\ldots+d_{k-1}$, hence

$$
\begin{aligned}
d_{0}^{*} & =\left[-\lambda_{n}+n-1-2\left(r_{1}+\ldots+r_{k-1}\right)-\left(d_{1}+\ldots+d_{k-1}\right)\right]-2 r_{k}+n+1 \\
& =-\lambda_{n}-\left(d_{1}+\ldots+d_{k-1}\right)=-d_{0}-d_{1} \ldots-d_{k-1} .
\end{aligned}
$$

It remains to prove that if $S_{i}$ corresponds to $\mathcal{T}_{i}$, then the dual plot $S_{i}^{*}$ corresponds to the mirrored tree $\mathcal{T}_{i}^{*}$. We induct on the rank of the sector. The 
case $r_{k}=1$ is clear. If $[a, b]$ is a sector, then $\lambda(a)=\boxplus$ and $\lambda(b)=\boxminus$. According to proposition 21.2 the dual plot is obtained by first exchanging $\boxplus$ and $\boxminus$ and then reflecting $s \mapsto 1-s$. Hence the dual plot of $S_{i}^{*}$ is obtained (ignoring distances) by keeping the outer labels $\boxplus$ and $\boxminus$ of the sector and dualising the plot of the inner segment $[a+1, b-1]$. This corresponds to keeping the root of the tree $\mathcal{T}_{i}$ and calculating the dual of the forest of the inner trees obtained from $\mathcal{T}_{i}$ by removing the root of $\mathcal{T}_{i}$. More precisely: The tree to the dual plot $S_{i}^{*}$ is obtained by taking the grafting product of the inner subtrees corresponding to the dual of the plot of the inner segment. The interval $[a+1, b-1]$ is a segment consisting of sectors $\tilde{S}_{1} \ldots \tilde{S}_{l}$ corresponding to the trees $\tilde{\mathcal{T}}_{1} \ldots \tilde{\mathcal{T}}_{l}$. Dualising the inner segment yields by induction the forest $\tilde{\mathcal{T}}_{l}^{*} \ldots \tilde{\mathcal{T}}_{1}^{*}$ since the ranks of inner sectors are smaller than the rank of $S_{i}$. Hence the tree corresponding to $S_{i}^{*}$ is obtained by grafting the forest $\tilde{\mathcal{T}}_{l}^{*} \ldots \tilde{\mathcal{T}}_{1}^{*}$. This is just the definition of the mirror image of $\mathcal{T}_{i}$.

Example. Consider the irreducible representation $[11,9,9,5,3,3,3]$ in $\mathcal{R}_{7}$. It has sector structure $S_{1}=[-3,4], S_{2}=[7,10]$ and $S_{3}=[11,12]$ with distances $d_{0}=3, d_{1}=2$ and $d_{2}=0$. The associated spaced forest is

$$
d_{0}=3
$$

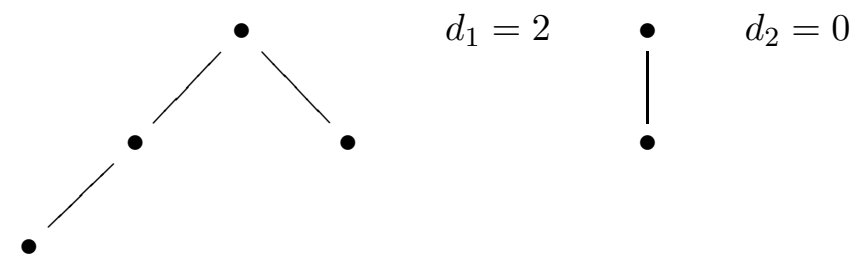

The dual is the representation $[1,1,0,0,-4,-4,-5]$ with sectors (from left to right) $S_{3}^{*}=[-11,-10], S_{2}^{*}=[-9,-6]$ and $S_{1}^{*}=[-3,4]$ with associated spaced forest
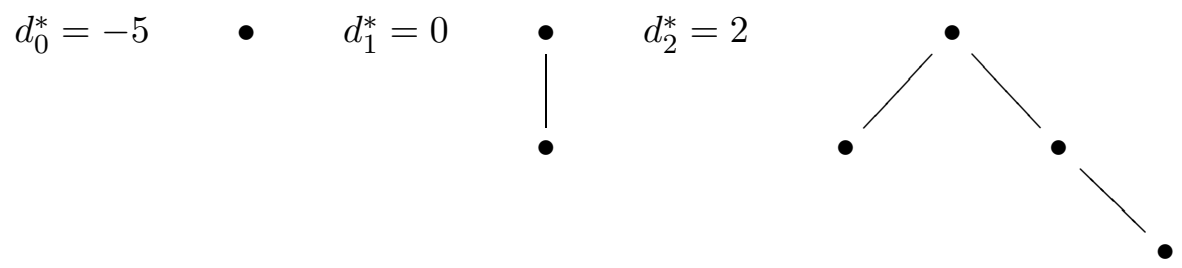

\section{Cohomology I}

In corollary 20.5 we have seen that in the situation of the melting algorithm one obtains surjective maps $H^{i}(p): H^{i}(\mathbb{A}) \rightarrow H^{i}(L)$ for all $i \in \mathbb{Z}$. For $K=\operatorname{Ker}(p: \mathbb{A} \rightarrow L)$ we therefore get exact sequences

$$
0 \rightarrow H^{i}(K) \rightarrow H^{i}(\mathbb{A}) \rightarrow H^{i}(L) \rightarrow 0
$$

for all integers $i$. Hence, if in addition $H^{i}(\mathbb{A})=0$ and $H^{i}(L)=0$ vanish for all $i \neq 0$, then $H^{i}(K)=0$ holds for all $i \neq 0$. Then $K / L \cong A$ implies 
$H^{i}(A)=0$ for $i \neq-1,0$. Suppose, the same conditions are satisfied for $\mathbb{A}^{\vee}$ as well. Then also $H^{i}\left(A^{\vee}\right)=0$ holds for $i \neq-1,0$. Then, by duality $H^{i}(A)^{\vee} \cong H^{-i}\left(A^{\vee}\right)$, the cohomology modules $H^{i}(A)$ vanish for $i \neq 0$. This proves

Proposition 22.1. For irreducible basic modules $V=\left[\lambda_{1}, \ldots, \lambda_{n-1}, 0\right]$ in $\mathcal{R}_{n}$ the cohomology modules $H^{i}(V)$ vanish for all $i \neq 0$.

Proof. We use induction with respect to the degree $p=p(\lambda)=\sum_{i} \lambda_{i}$, where $\lambda_{i}$ for $i=1, . ., n$ denote the coefficients of the weight vector. By induction assume the assertion holds for all irreducible basic modules of degree $<p$. For $V$ of degree $p$ by the melting algorithm there exists an irreducible basic module $L$ of degree $p-1$ and $\mathbb{A}$ with layer structure $(L, A, L)$ such that $A=V \oplus A^{\prime}$, where $A^{\prime}$ is a direct sum of irreducible basic modules of degree $<p$. Since $H^{i}(L)=0$ for $i \neq 0, H^{i}(\mathbb{A})=0$ for $i \neq 0$ now follows from lemma 20.4. The same applies for the dual modules $\mathbb{A}^{\vee}$ and $L^{\vee}$. Indeed the dual module of a basic irreducible module is basic irreducible again with the same degree $<p-1$ (lemma 21.4) using $\sum_{i} \lambda_{i}=\sum_{i} \lambda_{i}^{*}$. Hence the remarks preceding proposition 22.1 imply $H^{i}(A)=0$ for $i \neq 0$. Since $V$ is a direct summand of $A$, this proves our assertion.

\section{Cohomology II}

We calculate the Z-grading of $D S(L)$ for maximal atypical irreducible $L$. The case of general $L$ is treated in section 24 .

Proposition 23.1. For maximal atypical irreducible $L(\lambda)$ in $\mathcal{R}_{n}$ with weight $\lambda$, normalized so that $\lambda_{n}=0$, suppose $\lambda$ has sectors $S_{1}, . ., S_{i}, . ., S_{k}$ (from left to right). Then the constituents $L\left(\lambda_{i}\right)$ of $D S(L(\lambda))$ for $i=1, \ldots, k$ have sectors $S_{1}, . ., \partial S_{i}, . . S_{k}$, and the cohomology of $L(\lambda)$ can be expressed in terms of the added distances $\delta_{1}, \ldots, \delta_{k}$ between these sectors as follows:

$$
H^{\bullet}(L(\lambda))=\bigoplus_{i=1}^{k} L\left(\lambda_{i}\right)\left\langle-\delta_{i}\right\rangle .
$$

Example. We know by the main theorem that $D S([6,4,4,1])=\Pi[3,3,0] \oplus$ $\Pi[6,4,0] \oplus \Pi[6,4,4]$. The proposition above tells us the $\mathbf{Z}$-grading using

$$
D S(V)=\bigoplus_{\ell \in \mathbf{Z}} \Pi^{\ell}\left(H^{\ell}(V)\right) .
$$

In this example $d_{0}=1, d_{1}=2$ and $d_{2}=0$. The summand $L\left(\lambda_{i}\right)$ is obtained by differentiating the $i$-th sector in the plot associated to $\lambda$, hence $L\left(\lambda_{1}\right)=$ $[6,4,4], L\left(\lambda_{2}\right)=[6,4,0]$ and $L\left(\lambda_{3}\right)=[3,3,0]$. We obtain

$$
H^{\bullet}([6,4,4,1])=[6,4,4]\langle-1\rangle \oplus[6,4,0]\langle-3\rangle \oplus[3,3,0]\langle-3\rangle .
$$


Proof. In the special case where all distances vanish $d_{1}=\cdots=d_{k}=0$, i.e. in case where the plot of $\lambda$ has only one segment, the assertion of the proposition has been shown in proposition 22.1. We then prove the general case of nonvanishing distances by induction with respect to ( $n$ and) the lexicographic ordering used for algorithm I. This means: We prove proposition 23.1 recursively for $L^{u p}$, thereby assuming that we already know the cohomology degrees of $L_{r}^{\text {down}}, L$ and $L^{\text {aux }}$ (using the notations of algorithm I). First recall the notations used for algorithm I:

$$
\begin{gathered}
L=\left(S_{1} \cdots S_{j-1}\right) \leftarrow \text { distance } d_{j} \rightarrow\left(S_{j} S_{j+1} \cdots S_{k}\right) \leftarrow \text { distance } d_{k} \rightarrow \ldots \\
L^{u p}=\left(S_{1} \cdots S_{j-1}\right) \leftarrow \text { dist. }\left(d_{j}+1\right) \rightarrow \int\left(S_{j+1} \cdots S_{k}\right) \leftarrow \text { dist. }\left(d_{k}-1\right) \rightarrow \ldots
\end{gathered}
$$

for a sector $S_{j}$ with $r\left(S_{j}\right)=1$ supported at $i \in \mathbb{Z}$. Recall $\mathbb{A}=(L, A, L)$ with

$$
A=L^{u p} \oplus \bigoplus_{r=1}^{k} L_{r}^{\text {down }} .
$$

Furthermore $D S(L)=\Pi^{m_{\text {aux }}} L^{\text {aux }} \oplus \bigoplus_{\mu=1}^{s} \Pi^{m_{\mu}} \tilde{L}_{\mu}$ for $D S(\mathbb{A})=\bigoplus_{\mu=1}^{s} \tilde{\mathbb{A}}_{\mu}$ and $\tilde{\mathbb{A}}_{\mu}=\left(\tilde{L}_{\mu}, \tilde{A}_{\mu}, \tilde{L}_{\mu}\right)$ such that the derivative $d(A)$ of $A$ is

$$
d(A)=\tilde{A}+2(-1)^{i+n-1} L^{\text {aux }}
$$

in $K_{0}\left(\mathcal{R}_{n}\right)$. Obviously $D S\left(L^{u p}\right)$ has the summands

$$
\begin{aligned}
& \left.\bigoplus_{\nu=1}^{j-1}\left(S_{1} \cdots \partial S_{\nu} \cdots S_{j-1}\right) \ldots\left(d_{j-1}+1\right) \ldots S \ldots\left(d_{k}-1\right) \ldots S_{k+1} \cdots\right) \\
& \left.\bigoplus_{\nu>k}\left(S_{1} \cdots S_{j-1}\right) \ldots\left(d_{j-1}+1\right) \ldots S \ldots\left(d_{k}-1\right) \ldots S_{k+1} \cdots \partial S_{\nu} \cdots\right)
\end{aligned}
$$

and

$$
L^{a u x}=\left(S_{1} \cdots S_{j-1}\right) \ldots\left(d_{j-1}+2\right) \ldots\left(S_{j+1} \cdots S_{k}\right) \ldots\left(d_{k}\right) \ldots S_{k+1} \cdots .
$$

This immediately implies the next

Lemma 23.2. The following holds

(1) $D S\left(L^{u p}\right) \subseteq L^{\text {aux }} \oplus D S(L)^{u p}$.

(2) None of the summands of $D S\left(L^{u p}\right)$ different from $L^{\text {aux }}$ is contained in $D S(L)$.

(3) $L^{\text {aux }}$ is not a summand of $\bigoplus_{\mu} \tilde{A}_{\mu}$.

Proof. The last assertion holds, since the constituents of $\bigoplus_{\mu} \tilde{A}_{\mu}$ are obtained from $\tilde{L}_{\mu}$ by moves. It can be checked that $L^{a u x}$ can not be realized in this way.

The $d_{j-1} \pm 1$ alternative. By the induction assumption $H^{\bullet}(L)$ contains $L^{a u x}$ with multiplicity 1 , and $L^{a u x}$ appears in cohomology at the degree 
$d_{j-1}$. To determine $H^{i}\left(L^{u p}\right) \subseteq H^{i}(A)$ we may use step 11) of the proof of theorem 19.3. It easily implies by a small modification of the arguments that

$$
H^{i}(A)=\bigoplus_{m_{\mu}=-i} \tilde{A}_{\mu} \oplus H^{i-1}(L) / H^{i-1}\left(\bigoplus_{\mu} \tilde{L}_{\mu}\right) \oplus \operatorname{Kern}\left(H^{i+1}(L) \rightarrow H^{i+1}(K)\right) .
$$

Since $H^{\bullet}(L) /\left(\bigoplus_{\mu} \tilde{L}_{\mu}\right) \cong L^{\text {aux }}$ by lemma 20.1 and since

$$
H^{i-1}(L) /\left(\bigoplus_{\mu=1-i} \tilde{L}_{\mu}\right) \cong L^{a u x}
$$

for $i-1=d_{j-1}$ by the induction assumption, we get

$$
\operatorname{Kern}\left(H^{\bullet}(L) \rightarrow H^{\bullet}(K)\right)=L^{\text {aux }},
$$

and this implies

$$
\operatorname{Kern}\left(H^{i+1}(L) \rightarrow H^{i+1}(K)\right)=L^{a u x}
$$

for $i+1=d_{j-1}$. In other words $D S(A)=\tilde{A}+2 \cdot L^{\text {aux }}$ and the two copies of $L^{\text {aux }}$ occur in the two possible cohomology degrees

$$
d_{j-1} \pm 1 \text {. }
$$

Continuation of the prooffor proposition 23.1. By lemma 23.2 the cohomology degree of the constituents of $H^{\bullet}\left(L^{u p}\right)$ that appear in

$$
\tilde{A}_{\mu} \subseteq \bigoplus_{m_{\mu}=-i} \tilde{A}_{\mu}
$$

can be immediately read of from the degrees $m_{\mu}$, i.e. from the cohomology degrees of $\tilde{L}_{\mu}$ in $H^{\bullet}(L)$. These degrees are known by the induction assumption. This easily proves proposition 23.1 for all constituents $L\left(\lambda_{i}\right)$ of $H^{\bullet}\left(L^{u p}\right)$ that are not isomorphic to $L^{\text {aux }}$. Indeed, according to our claim the cohomological degrees for the constituents $L\left(\lambda_{i}\right) \approx L^{a u x}$ of $H^{\bullet}\left(L^{u p}\right)$ are given by

$$
0, \cdots, 0, d_{j-1}+1, d_{j-1}+d_{k}, \cdots,
$$

and the summand $L^{\text {aux }}$ should occur in degree $d_{j-1}+1$. The cohomology of $H^{\bullet}(L)$ on the other hand is concentrated in the degrees

$$
0, \cdots, 0, d_{j-1}, d_{j-1}+d_{k}, \cdots
$$

with the summand $L^{a u x}$ corresponding to degree $d_{j-1}$. All summands $\not$ $L^{\text {aux }}$ precisely match, so this proves proposition 23.1 for all constituents of $H^{\bullet}\left(L^{u p}\right)$ except for $L^{a u x}$.

It remains to determine the cohomology degree of $L^{\text {aux }} \subseteq H^{\bullet}\left(L^{u p}\right)$. As already explained, the summand $L^{a u x}$ occurs in degree $d_{j-1}-1$ or $d_{j-1}+1$. So to show that $L^{a u x}$ occurs in $H^{\bullet}\left(L^{u p}\right)$ for degree $d_{j-1}+1$, it now suffices by the $d_{j-1} \pm 1$ alternative to show that $L^{\text {aux }}$ occurs in $H^{\nu}\left(\bigoplus_{r} L_{r}^{\text {down }}\right)$ in the 
degree $\nu=d_{j-1}-1$. Indeed $L^{\text {aux }}$ appears in $D S\left(L^{\text {down }}\right)=\bigoplus_{\nu} H^{\nu}\left(L^{\text {down }}\right)$ for $L^{\text {down }}:=L_{j}^{\text {down }}$. This follows from the structure of the sectors of

$$
L^{\text {down }}=\int\left(S_{1} \cdots S_{j-1}\right) \boxminus \ldots\left(d_{j-1}-1\right) \ldots \boxminus_{i+1} S_{j+1} \cdots S_{k}
$$

and the induction assumption. It gives the degree $d_{j-1}-1$, for $d_{j-1} \geq 1$, respectively in degree $d_{j-1}-1=-1$, for $d_{j-1}=0$, for the summand $L^{\text {aux }}$ in $H^{\bullet}\left(L^{\text {down }}\right)$. Hence

$$
L^{a u x} \subseteq H^{d_{j-1}+1}\left(L^{u p}\right),
$$

which completes the proof of proposition 23.1.

\section{Cohomology III}

The cohomology of an $i$-atypical $L$ can be calculated in the same way using the normalised block equivalence $\phi_{n}^{i}$ of section 15. We call an irreducible module $L$ of atypicality $i \phi$-basic if $\phi_{n}^{i}(L)$ is basic in $\mathcal{R}_{i}$. These will replace the basic modules in the proof of proposition 22.1. The unique mixed tensor in a block of atypicality $i$ replaces the trivial representation.

Proposition 24.1. For irreducible $\phi$-basic modules $V$ in $\mathcal{R}_{n}$ the cohomology modules $H^{i}(V)$ vanish for all $i \neq 0$.

Proof. The remarks preceeding proposition 22.1 are valid. By lemma 14.4 the cohomology of the mixed tensor $L(\lambda)$ is concentrated in one degree, and by lemma 10.2 this degree is zero since $\lambda_{n}=0$. Since $\phi_{n}^{i}(L(\lambda))=\mathbf{1}$, we induct as in the proof of 22.1 on the sum $p=\sum_{i} \lambda_{i}$ of the coefficients of $\phi_{n}^{i}(L)$. The rest of the proof works verbatim. Note that the dual of a $\phi$-basic module is $\phi$-basic again of the same degree using $\phi_{n}^{i}(L)^{\vee}=\phi_{n}^{i}\left(L^{\vee}\right)$ of lemma 21.3 and lemma 21.4.

We can now copy the proof of proposition 23.1 to obtain the next statement. Here the added distances $\delta_{i}$ are the distances in the plot $\phi(\lambda)$ associated to $\lambda$ in section 18 .

Proposition 24.2. For irreducible $L(\lambda)$ in $\mathcal{R}_{n}$ with weight $\lambda$, normalized so that $\phi_{n}^{i}(L(\lambda))=\left[\lambda_{1}^{\phi}, \ldots, \lambda_{i}^{\phi}\right]$ satisfies $\lambda_{i}^{\phi}=0$, suppose $\lambda$ has sectors $S_{1}, . ., S_{j}, . ., S_{k}$ (from left to right). Then the constituents $L\left(\lambda_{j}\right)$ of $D S(L(\lambda)$ ) for $j=1, \ldots, k$ have sectors $S_{1}, . ., \partial S_{j}, . . S_{k}$, and the cohomology of $L(\lambda)$ can be expressed in terms of the added distances $\delta_{1}, \ldots, \delta_{k}$ between these sectors as follows:

$$
H^{\bullet}(L(\lambda))=\bigoplus_{j=1}^{k} L\left(\lambda_{j}\right)\left\langle-\delta_{j}\right\rangle .
$$




\section{THE FOREST FORMULA}

Recall the functor $D S_{n, 0}: T_{n} \rightarrow T_{0}=$ svec $_{k}$ with its decomposition $D S_{n, 0}(V)=\bigoplus_{\ell \in \mathbb{Z}} D_{n, 0}^{\ell}(V)[-\ell]$ for objects $V$ in $T_{n}$ and objects $D_{n, 0}^{\ell}(V)$ in $\operatorname{svec}_{k}$. For $V \in T_{n}$ we define the Laurent polynomial

$$
\omega(V, t)=\sum_{\ell \in \mathbb{Z}} \operatorname{sdim}\left(D_{n, 0}^{\ell}(V)\right) \cdot t^{\ell}
$$

as the Hilbert polynomial of the graded module $D S_{n, 0}^{\bullet}(V)=\bigoplus_{\ell \in \mathbb{Z}} D_{n, 0}^{\ell}(V)$. Since $\operatorname{sdim}(W[-\ell])=(-1)^{\ell} \operatorname{sim}(W)$ and $V=\bigoplus D_{n, 0}^{\ell}(V)[-\ell]$ holds, the formula

$$
\operatorname{sim}(V)=\omega(V,-1)
$$

follows. For $V=\operatorname{Ber}_{n}^{i}$

$$
\omega\left(\operatorname{Ber}_{n}^{i}, t\right)=t^{n i}
$$

Indeed, $H^{\ell}\left(\operatorname{Ber}_{n}^{i}\right)=0$ for $\ell \neq i$ and $H^{\ell}\left(B e r_{n}^{i}\right)=B e r_{n-1}^{i}$ for $\ell=i$ implies $D S\left(\operatorname{Ber}_{n}^{i}\right)=B e r_{n-1}^{i}[-i]$. If we apply this formula $n$-times and consider $B_{0}=\mathbf{1}$, we obtain $D S_{n, 0}\left(B e r_{n}^{i}\right)=D S^{n}\left(B e r_{n}^{i}\right)=\mathbf{1}[-n i]$ from the fact that $D S_{n, 0}(L)=D S^{n}(L)$ holds for simple objects $L$. This implies $D S_{n, 0}^{n i}\left(\operatorname{Ber}_{n}^{i}\right)=\mathbf{1}$ and that $D S_{n, 0}^{\ell}\left(B e r_{n}^{i}\right)$ is zero otherwise.

Since $D S_{n, 0}$ is a tensor functor, $\omega(M \otimes L, t)=\omega(M, t) \omega(L, t)$ holds. Hence

$$
\omega\left(\operatorname{Ber}_{n}^{i} \otimes L, t\right)=t^{n i} \cdot \omega(L, t) .
$$

Similar as in the proof of lemma 3.1 one shows

$$
\omega\left(V^{\vee}, t\right)=\omega\left(V, t^{-1}\right) .
$$

Let now $L=L(\lambda)$ be a maximal atypical irreducible representation in $\mathcal{R}_{n}$. Associated to its plot $\lambda$ we have the basic plot $\lambda_{b a s i c}$ and the numbers $d_{0}, \ldots, d_{k-1}$. Furthermore, let $S_{1} S_{2} \cdots S_{k}$ be the sector structure of $\lambda_{\text {basic }}$. For the degrees $r_{i}=r\left(S_{i}\right)$ we define the number

$$
D(\lambda)=\sum_{i=1}^{k} r_{i} \sum_{0 \leq j<i} d_{j}=\sum_{i=1}^{k} r_{i} \delta_{i},
$$

Recall $\delta_{i}=\sum_{\nu=0}^{i-1} d_{\nu}$ implies $\delta_{1} \leq \delta_{2} \leq \cdots \leq \delta_{k}$ and $\delta_{i} \in \mathbb{Z}^{k}$. Consider the vector $D$ with coordinates $\delta_{1}, \ldots, \delta_{k}$. Together with $\lambda_{\text {basic }}$ the knowledge of $D$ determines $\lambda$. For simplicity, we express this by writing $\lambda=D \times \lambda_{\text {basic }}$ in the following argument. With this notation, our proposition 23.1 gives for 
$D S(L)$ the following element in the Grothendieck group $K_{0}\left(\mathcal{R}_{n-1}\right) \otimes k[t]$

$$
D S\left(\left(\begin{array}{c}
\delta_{1} \\
\cdot \\
\delta_{i-1} \\
\delta_{i} \\
\delta_{i+1} \\
\cdot \\
\delta_{k}
\end{array}\right) \times\left(S_{1} \cdots S_{k}\right)_{\text {basic }}\right)=\sum_{i=1}^{k} t^{\delta_{i}} \cdot\left(\begin{array}{c}
\delta_{1}-1 \\
\cdot \\
\delta_{i-1}-1 \\
\delta_{i} \\
\delta_{i+1}+1 \\
\cdot \\
\delta_{k}+1
\end{array}\right) \times\left(S_{1} \cdots \partial S_{i} \cdots S_{k}\right)_{\text {basic }}
$$

where formally (and without loss of information) we replace the shifts $[-\nu]$ by $t^{\nu}$. In the following, we refer to this formula as the key formula. Now $\partial S_{i}$ may introduce new sectors in $\left(S_{1} \cdots \partial S_{i} \cdots S_{k}\right)_{b a s i c}$. So if we want to treat everything on an equal footing, we better count each sectors $S_{i}$ with the multiplicity $r_{i}$. This amounts to consider instead of the vector $D$ the new refined vector $\delta$ in $\mathbb{Z}^{n}$ with the coordinates

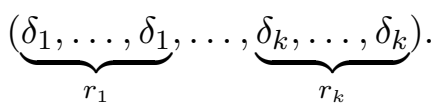

Then the number $D(\lambda)$ defined above is just the sum of the coordinates of this vector. With this new vector we have an analogous formula expressing $D S$ as above, where for the $i$-th summand on the right side one of the entries $\delta_{i}$ of $\delta$ has to be removed to obtain a vector in $\mathbb{Z}^{n-1}$. The right side is now of the correct form to enable the application of the formula for $D S$ to the right side again. Inductively, after $n$ steps this gives a complicated expression with at most $n$ ! summands. The number of summands depends only on $\lambda_{\text {basic }}$. Since the additional monomial term in $t$ obtained from each derivative is of the form $t^{\delta_{\nu} \pm s_{\nu}}$, for some shift factors $s_{\nu}$ not depending on $\delta$, and since in each summand all coordinate entries of $\delta$ will be finitely successively deleted after $n$ times applying $D S$, this vector disappears and each of these summands has the form

$$
t^{\sum_{i=1}^{k} r_{i} \delta_{i}} \cdot P(t) \times \emptyset
$$

for a certain Laurent polynomials $P(t)$ that depends on the specific summand and on $\lambda_{\text {basic }}$, but that does not depend on the coefficients $\delta_{1}, \ldots, \delta_{k}$. If we compare with the case $\delta_{1}=\ldots=\delta_{k}=0$, we therefore obtain the following translation formula:

$$
\omega(L(\lambda), t)=t^{D(\lambda)} \cdot \omega\left(L\left(\lambda_{b a s i c}\right), t\right) .
$$

This being said, we use that the basic plots of rank $n$ are in 1-1 correspondence with planar forests $\mathcal{F}$ with $n$ nodes $x \in \mathcal{F}$ as in sections 1621 and [Wei10]. For a planar forest, let $\# \mathcal{F}$ denote the number of its nodes. We visual each of the trees in a plain forest top down, i.e. with their root on the top of the tree. Then, for each node $x \in \mathcal{F}$ let $\mathcal{F}(x)$ denote the subtree of 
the tree containing $x$ with all nodes removed that are not below the node $x$. In this way the node $x$ becomes the root of the tree $\mathcal{F}(x)$ by definition. For a forest $\mathcal{F}$ we recursively define the quantum forest factorials

$$
[\mathcal{F}]_{t} !=\prod_{x \in \mathcal{F}}[\# \mathcal{F}(x)]_{t} \in \mathbb{Z}[t]
$$

using the following abbreviations: For the number $m=\# \mathcal{F}(x)$ of nodes in $\mathcal{F}(x)$ we define the quantum numbers

$$
[m]_{t}:=\frac{t^{m}-t^{-m}}{t-t^{-1}} .
$$

Clearly $[m]_{t}=(-1)^{m-1}[m]_{-t}=[m]_{t^{-1}}$. Obviously the tree factorial $\mathcal{T}$ ! of section 16 equals $[\mathcal{T}]_{1}$ !. Example: For the forest $\mathcal{F}$ that contains only one linear tree, the forest factorial $[\mathcal{F}]_{t}$ ! specializes to the quantum factorial $[n]_{t} !=\prod_{m=1}^{n}[m]_{t}$. For a planar forest $\mathcal{F}$, given as the union of trees $\mathcal{T}_{i}$ for $i=1, \ldots, k$ with $r_{i}$ nodes respectively, one has $[\mathcal{F}]_{t} !=\prod_{i=1}^{k}\left[\mathcal{T}_{i}\right]_{t} !$ and hence

$$
\text { (*) } \quad \frac{[\# \mathcal{F}]_{t} !}{[\mathcal{F}]_{t} !}=\frac{\left[\sum_{i} r_{i}\right]_{t} !}{\left[r_{1}\right]_{t} ! \cdots\left[r_{k}\right]_{t} !} \cdot \prod_{i=1}^{k} \frac{\left[\# \mathcal{T}_{i}\right]_{t} !}{\left[\mathcal{T}_{i}\right]_{t} !} .
$$

Observe, for a tree $\mathcal{T}$ the value $\frac{[\# \mathcal{T}]_{t} \text { ! }}{[\mathcal{T}]_{t} !}$ does not change under grafting, i.e. replacing $\mathcal{T}$ by a new tree with $\# \mathcal{T}+1$ nodes by putting a new root on top. Similar $\frac{[\# \mathcal{F}]_{t} !}{[\mathcal{F}]_{t} !}$ does not change under the grafting of the planar forest $\mathcal{F}$, that replaces $\mathcal{F}$ by a forest with a single tree with $\# \mathcal{T}+1$ nodes obtain by putting a new root on top of all trees connected to the old roots of the trees of $\mathcal{F}$.

Lemma 25.1. For irreducible maximal atypical representations $L=L(\lambda)$ in $\mathcal{R}_{n}$ we have the forest formula

$$
\omega(L, t)=t^{D(\lambda)} \cdot \frac{[n]_{t} !}{\left[\lambda_{\text {basic }}\right]_{t} !}
$$

where $\lambda_{\text {basic }}$ is viewed as the planar forest associated to $L$.

Proof. From the translation formula we may assume $\lambda=\lambda_{b a s i c}$. Let us first consider the simple case of basic representations $L$, where all sectors $S_{i}$ for $i=1, . ., k$ are intervals $I_{i}=\left[a_{i}, a_{i}+2 r_{i}-1\right]$ where the support of the plot $S_{i}$ is $\left[a_{i}, \ldots, a_{i}+r_{i}-1\right]$. The corresponding $\omega(L, t)$ then only depends on the ranks $r_{1}, \ldots, r_{k}$ of the sectors $S_{1}, \ldots, S_{k}$, hence will be denoted $\omega_{r_{1}, \ldots, r_{k}}(t)$ in the following. From the key formula then, for general basic $\lambda$ with sector structure $S_{1} \cdots S_{k}$ and $r_{i}=r\left(S_{i}\right)$, similarly to the translation formula we easily obtain the following generalized Leibniz formula

$$
\omega(L(\lambda), t)=\omega_{r_{1}, \ldots, r_{k}}(t) \cdot \prod_{i=1}^{k} \omega\left(L\left(S_{i}\right), t\right),
$$


where $L\left(S_{i}\right)$ denotes the irreducible basic maximal atypical representation in $\mathcal{R}_{r_{i}}$ whose plot is $S_{i}$ (up to a translation on the number line). Now each of these $S_{i}$ has a unique sector. For basic plots $S$ with a unique sector (like the $S_{i}$ ) the key formula obviously implies the following grafting formula

$$
\omega\left(L\left(S_{i}\right), t\right)=\omega(L(\partial S), t),
$$

where $L(\partial S)$ denotes the unique maximal atypical basic representation in $\mathcal{R}_{r(S)-1}$ whose plot is $\partial S$. So the forest attached to $L(S)$ is obtained by grafting the forest of $\partial S$.

It is clear that inductively the translation formula, the generalized Leibniz formula and the grafting formula determine the Laurent polynomials $\omega(L, t)$ for irreducible maximal atypical $L \in \mathcal{R}_{n}$ uniquely. Hence for the proof it suffices that the expression on the right side of the identity stated in lemma 25.1 satisfies the analogous formulas and that it holds for $n=1$. Indeed, our assertion is obvious for $n=1$. The translation formula and the grafting formula for the right side are also obvious. To check the generalized Leibniz formula, by the formula (*) from above it suffices to prove

$$
\omega_{r_{1}, \ldots, r_{k}}(t)=\frac{\left[\sum_{i} r_{i}\right]_{t} !}{\prod_{i=1}^{k}\left[r_{i}\right]_{t} !} .
$$

To this end it is helpful that for $a=r_{1}+\cdots+r_{i}$ and $b=r_{i+1}+\cdots+r_{k}$, by the key formula, also the following version of the generalized Leibniz formula holds

$$
\omega_{r_{1}, \ldots, r_{k}}(t)=\omega_{a, b}(t) \omega_{r_{1}, \ldots, r_{i}}(t) \omega_{r_{i+1}, \ldots, r_{k}}(t) .
$$

So it suffices to verify that $\omega_{a, b}(t)[a]_{t} ![b]_{t} !=[a+b]_{t} !$, which finally is proved by induction on $n=a+b$. For this notice that the key formula immediately implies the following generalized Pascal rule

$$
\omega_{a, b}(t)=t^{b} \cdot \omega_{a-1, b}(t)+t^{-a} \cdot \omega_{a, b-1}(t)
$$

for the generalized binomial coefficients $\omega_{a, b}(t)$. Indeed, the derivative of the two sectors $S_{1} S_{2}$ give $\partial S_{1} S_{2}$ with $d_{0}=0, d_{1}=1$ respectively $S_{1} \partial S_{2}$ with $d_{0}=-1, d_{1}=1$. Hence $D\left(\partial S_{1} S_{2}\right)=0 \cdot(a-1)+1 \cdot b=b$ and $D\left(S_{1} \partial S_{2}\right)=-a+0 \cdot(b-1)=-a$. Hence using the induction assumption, we already know $\omega_{a-1, b}(t)[a-1]_{t} ![b]_{t} !=[a+b-1]_{t} !$ and $\omega_{a, b-1}(t)[a]_{t} ![b-1]_{t} !=$ $[a+b-1]_{t}$ !. Hence the proof of the induction step finally amounts for the quantum numbers $[\mathrm{m}]_{t}$ to the following generalized additivity

$$
[a+b]_{t}=t^{b} \cdot[a]_{t}+t^{-a} \cdot[b]_{t}
$$

that is easily verified. This completes the proof. 
Since $[n]_{t}$ ! and $\left[\lambda_{\text {basic }}\right]_{t}$ ! are products of certain Laurent polynomials $[m]_{t}$ for integers $m$, the forest formula implies $\omega(L,-t)= \pm \omega(L, t)$. The forest formula also gives $\omega(L, 1)>0$. Since $\omega(L,-1)=\operatorname{sdim}(L)$, hence $\omega(L,-t)=\operatorname{sign}(\operatorname{sim}(L)) \cdot \omega(L, t)$. Recall that for irreducible $L$ in $\mathcal{R}_{n}$ we defined a sign $\varepsilon(L)$ and that the sign of $\operatorname{sim}(L)$ is $\varepsilon(L)$, as shown in section 16 and also in [Wei10]. Finally, the forest formula also implies $\omega\left(L, t^{-1}\right) / \omega(L, t)=t^{-2 D(\lambda)}$ for $L=L(\lambda)$. Hence we obtain

Lemma 25.2. For irreducible (maximal atypical) $L=L(\lambda)$ in $\mathcal{R}_{n}$ one has the formulas $\omega\left(L^{\vee}, t\right)=\omega\left(L, t^{-1}\right)=t^{-2 D(\lambda)} \omega(L, t)$ and

$$
\omega(L,-t)=\varepsilon(L) \cdot \omega(L, t) .
$$

Example. For $S^{n-1+d}$ in $\mathcal{R}_{n}$ and for integers $d \geq 0$

$$
\omega\left(S^{n-1+d}, t\right)=t^{d-n+1}+t^{d-n+3}+\cdots+t^{d+n-1}=t^{d} \cdot \omega\left(S^{n-1}, t\right) .
$$

Lemma 25.3. For irreducible max. atypical representations $L=L(\lambda)$ in $\mathcal{R}_{n}$ the Laurent polynomial $\omega(L, t)$ has degree $p(\lambda)=\sum_{i=1}^{n} \lambda_{i}$ in the sense that

$$
\omega(L, t)=t^{p(\lambda)}+\sum_{\ell<p(\lambda)} a_{\ell} \cdot t^{\ell}
$$

Proof. This follows from the key formula. Indeed its $i$-th summand gives rise to shifts by $\delta_{i+1}+1, \ldots, \delta_{k}+1$. To determine the highest cohomology degree of $L(\lambda)$ one has to look for the maximal contributions from all these shifts. Each time we apply $D S$, the maximal contribution is obtained from the first summand $i=1$. Hence the highest $t$-power arises from the first summands of the key formula each times we apply $D S$ ( $n$-times), in other words by applying the derivative $\partial$ each time to the leftmost sector. In particular the highest $t$-power of $\omega(L(\lambda), t)$ is $t^{\delta_{1}}$ times the highest $t$-power of $\omega(\bar{L}, t)$ for the representation $\bar{L} \in T_{n-1}$ associated to the plot $\left(\partial S_{1}\right) S_{2} \cdots S_{k}$ with the new vector $\delta=\left(\delta_{1}, \ldots, \delta_{1}, \delta_{2}+1, \cdots, \delta_{k}+1\right)$ with one copy of $\delta_{1}$ deleted. Now it is not hard to see, by unraveling the weight associated to this spaced forest, that the associated representation $\bar{L}$ is the highest weight module $L(\bar{\lambda})$ in the sense of Lemma 10.2. Therefore, the highest $t$-power of $\omega(L, t)$ is $t^{\lambda_{n}}$ times the highest $t$-power of $\omega(\bar{L}, t)$. In other words $\operatorname{deg}_{t}(\omega(L, t))=\lambda_{n}+\operatorname{deg}_{t}(\omega(\bar{L}, t))$. By induction on $n$ hence $\operatorname{deg}_{t}(\omega(L, t))=\lambda_{n}+p(\bar{\lambda})=p(\lambda)$.

Therefore the forest formula implies

Corollary 25.4. For irreducible maximal atypical representations $L=$ $L(\lambda)$ in $\mathcal{R}_{n}$ one has the formula $D(\lambda)=p(\lambda)-p\left(\lambda_{\text {basic }}\right)$.

By lemma 25.3 and 25.2 furthermore 
Corollary 25.5. For irreducible maximal atypical representations $L=$ $L(\lambda)$ in $\mathcal{R}_{n}$

$$
\omega(L, t)=t^{p(\lambda)}+\sum_{q(\lambda)<\ell<p(\lambda)} a_{\ell} \cdot t^{\ell}+t^{q(\lambda)}
$$

holds for $p(\lambda)-q(\lambda)=p(\lambda)+p\left(\lambda^{\vee}\right)=2 \cdot p\left(\lambda_{\text {basic }}\right)$.

Proof. From the forest formula and lemma 25.2 we obtain

$$
q(\lambda)=D(\lambda)+q\left(\lambda_{\text {basic }}\right)=D(\lambda)-p\left(\lambda_{\text {basic }}\right)
$$

Hence $p(\lambda)-q(\lambda)=2 p\left(\lambda_{\text {basic }}\right)$. Since $\omega\left(L^{\vee}, t\right)=\omega\left(L, t^{-1}\right)$, we obtain $p\left(\lambda^{\vee}\right)=-q(\lambda)=-D(\lambda)+p\left(\lambda_{\text {basic }}\right)$. Combined with corollary 25.4 this last formula gives $p(\lambda)+p\left(\lambda^{\vee}\right)=2 p\left(\lambda_{\text {basic }}\right)$.

\section{I-MODULE STRUCTURE ON THE COHOMOLOGY $H_{D S_{n}}^{\bullet}$}

In this section we show that the cohomology of the operator $D S_{n, 0}$ is a graded module under the invariant algebra $I=\Lambda^{\bullet}\left(\mathfrak{p}_{-1}\right)^{H}$ defined below. As an application we compute the cohomology and the Hilbert polynomial of a maximal atypical Kac module $V(\lambda)$ for the operator $D S_{n, 0}$. We also show that the projection of $V(\lambda)$ to $L(\lambda)$ induces a map on the $D S_{n, 0}$-cohomology which vanishes except in the top degree $p(\lambda)$. Note that it does not make sense to consider the Hilbert polynomial $\omega(V, t)$ for $V=V(\lambda)$ and the Dirac operator $D$ since any Kac module is in the kernel of $H_{D}$.

The tensor functor associated to an element $x$ in $X=\left\{x \in \mathfrak{g}_{1} \mid[x, x]=0\right\}$ only depends by [DS05] on the $G_{0}$-orbit on $X$. We therefore work in this section with the operator $D S_{n}$ associated to the action of the element

$$
\mathbb{D}=\left(\begin{array}{cc}
0 & i d_{n} \\
0 & 0
\end{array}\right)
$$

which is clearly in the same $G_{0}$-orbit as our usual choice of $x \in \mathfrak{g}_{1}$ with 1's in the anti-diagonal. It defines a (graded) tensor functor $D S_{n}: T_{n} \rightarrow T_{0}$ which is isomorphic to $D S_{n, 0}$.

Notations and conventions.

- Let $H$ denote $G l(n)$ diagonally embedded into $G_{0}=G l(n) \times G l(n)$ via $g \mapsto \operatorname{diag}(g, g) \in G_{0}$. Then $\operatorname{Lie}(H) \cong \mathfrak{g l}(n)$. 
- We consider the subalgebra $\mathfrak{p} \subset \mathfrak{g}$ with grading $\mathfrak{p}=\mathfrak{p}_{-} \oplus \mathfrak{p}_{0} \oplus \mathfrak{p}_{+}$

$$
\begin{aligned}
\mathfrak{p}_{-} & =\left\{\left(\begin{array}{ll}
0 & 0 \\
x & 0
\end{array}\right) \mid x \in \mathfrak{g l}(n)\right\} \\
\mathfrak{p}_{0} & =\left\{\left(\begin{array}{cc}
x & 0 \\
0 & x
\end{array}\right) \mid x \in \mathfrak{g l}(n)\right\}=\operatorname{Lie}(H) \\
\mathfrak{p}_{+} & =\left\{\left(\begin{array}{cc}
0 & i d_{n} \\
0 & 0
\end{array}\right)\right\} .
\end{aligned}
$$

- Recall that the restriction of the Kac module $V(\lambda)$ to $\mathfrak{p}$ is given by

$$
\left.V(\lambda)\right|_{\mathfrak{p}}=\Lambda^{\bullet}\left(\mathfrak{p}_{-}\right) \otimes L_{0}(\lambda)
$$

where $L_{0}(\lambda)$ is the irreducible $\mathfrak{g}_{0}$-module $L_{0}(\lambda)$ trivially extended to the parabolic subalgebra of upper triangular block matrices.

- We write in this section $\rho^{\vee} \otimes \rho$ for the irreducible representation $L_{0}(\lambda)$ of $\mathfrak{g}_{0}$ which is given by the external tensor product of the irreducible $\mathfrak{g l}(n)$-representation $\rho^{\vee}$ of weight $\left(\lambda_{1}, \ldots, \lambda_{n}\right)$ with its dual of weight $\left(-\lambda_{n}, \ldots,-\lambda_{1}\right)$. If viewed as a representation of $H \subset$ $G_{0}$ this becomes $\rho^{\vee} \otimes \rho \cong \operatorname{End}(\rho)$. In this notation $V(\lambda)=V\left(\rho^{\vee} \otimes \rho\right)$ and $L(\lambda)=L\left(\rho^{\vee} \otimes \rho\right)$.

- The tensor product $\rho^{\vee} \otimes \rho$ contains the trivial representation with multiplicity 1 . We call a vector in this subspace an $H$-spherical vector. In this sense $L_{0}(\lambda)$ has an $H$-spherical vector if and only if $\lambda$ is maximal atypical.

The action of the generator $\mathbb{D}$ of $\mathfrak{p}_{+}$on $\mathfrak{p}$-modules induces the operator denoted $D S_{n}$ in the following. In particular $\mathbb{D}$ acts on $\mathfrak{p}$ and the ideal $\mathfrak{p}_{-} \oplus \mathfrak{p}_{0}$ via the adjoint representation. Notice $\operatorname{Lie}(H)$ acts on $\mathfrak{p}_{0} \cong \operatorname{Lie}(H)$ by the adjoint representation of $\operatorname{Lie}(H)$ and on $\mathfrak{p}_{-}$by the adjoint action of $\mathfrak{p}$ such that the map

$$
\left(\begin{array}{ll}
0 & 0 \\
x & 0
\end{array}\right) \mapsto\left(\begin{array}{cc}
x & 0 \\
0 & x
\end{array}\right)
$$

is a $\operatorname{Lie}(H)$-linear isomorphism $\mathfrak{p}_{-} \cong \mathfrak{p}_{0}$ inducing a canonical identification $\Lambda^{\bullet}\left(\mathfrak{p}_{-}\right) \cong \Lambda^{\bullet}\left(\mathfrak{p}_{0}\right)$ of $H$-modules.

The universal enveloping algebra $U(\mathfrak{p})$ of $\mathfrak{p}$ contains the universal enveloping algebras $U\left(\mathfrak{p}_{-} \oplus \mathfrak{p}_{0}\right)$ and $U\left(\mathfrak{p}_{-}\right)$as subalgebras. For $\theta \in \Lambda^{\bullet}\left(\mathfrak{p}_{-}\right) \cong U\left(\mathfrak{p}_{-}\right)$ the supercommutator $[\mathbb{D}, \theta]$ is contained in $U\left(\mathfrak{p}_{-} \oplus \mathfrak{p}_{0}\right)$. For a basis $x_{i j}$ of $\operatorname{Lie}(H)$ it has the form $[\mathbb{D}, \theta]=\theta_{0}+\theta_{1}$ with $\theta_{1}=\sum_{i, j=1}^{n} \theta_{i j} \cdot x_{i j}$ for uniquely defined elements $\theta_{0}, \theta_{i j} \in \Lambda^{\bullet}\left(\mathfrak{p}_{-}\right) \cong U\left(\mathfrak{p}_{-}\right)$.

We now consider $V(\lambda)$ as a $\mathfrak{p}$-module

$$
\left.V\left(\rho^{\vee} \otimes \rho\right)\right|_{\mathfrak{p}}=\Lambda^{\bullet}\left(\mathfrak{p}_{-}\right) \otimes \operatorname{End}(\rho) .
$$


Lemma 26.1. The operator $D S_{n}$ induces the Lie algebra homology differential $\delta$ on the Chevalley-Eilenberg complex $\Lambda^{\bullet}\left(\mathfrak{p}_{-}\right) \otimes \operatorname{End}(\rho)$.

We shortly recall the definition. For a Lie algebra $\mathfrak{g}$ and a $\mathfrak{g}$-module $V$ we consider the complex with $p$-th entry $V_{p}(\mathfrak{g}, V)=\Lambda^{p}(\mathfrak{g}) \otimes V$ and differential $\delta: V_{p}(\mathfrak{g}, V) \rightarrow V_{p-1}(\mathfrak{g}, V)$ given for $p \geq 2$ by $\delta\left(x_{1} \wedge \ldots \wedge x_{p} \otimes v\right)=\theta_{0} \otimes v+\theta_{1}(v)$ for $x_{1}, \ldots, x_{p} \in \mathfrak{g}$ where

$$
\begin{aligned}
\theta_{0} \otimes v & =\left(\sum_{\mu<\nu}(-1)^{\mu+\nu}\left[x_{\mu}, x_{\nu}\right] \otimes x_{1} \wedge \ldots \wedge \hat{x}_{\mu} \wedge \ldots \wedge \hat{x}_{\nu} \wedge \ldots \wedge x_{p}\right) \otimes v \\
\theta_{1}(v) & =\sum_{\nu=1}^{p}\left((-1)^{\nu+1} x_{1} \wedge \ldots \wedge \hat{x}_{\nu} \wedge \ldots \wedge x_{p}\right) \otimes x_{\nu}(v) .
\end{aligned}
$$

Proof. $\mathbb{D}$ acts on an element $x_{1} \wedge \ldots \wedge x_{r} \otimes \varphi$ in $\Lambda^{\bullet}\left(\mathfrak{p}_{-}\right) \otimes \operatorname{End}(\rho)$ for $x_{1}, \ldots, x_{r} \in$ $\mathfrak{p}_{-}$and $\varphi \in \operatorname{End}(\rho)$ as

$$
\begin{aligned}
& \mathbb{D}\left(x_{1} \wedge \ldots \wedge x_{r} \otimes \varphi\right) \\
& =\sum \pm x_{1} \wedge \ldots \mathbb{D}\left(x_{i}\right) \wedge \ldots x_{r} \otimes \varphi \pm \sum x_{1} \wedge \ldots \wedge x_{r} \otimes \mathbb{D}(\varphi)
\end{aligned}
$$

with $\mathbb{D}\left(x_{i}\right) \in \mathfrak{p}_{0}$. The second sum vanishes since $\mathbb{D}(\varphi)=0$ for all $\varphi \in$ $\operatorname{End}(\rho)$ by definition of the Kac module. We now evaluate the first sum. $\mathbb{D}$ acts on an element in $\mathfrak{p}_{-1}$ by the supercommutator

$$
\left[\left(\begin{array}{cc}
0 & i d_{n} \\
0 & 0
\end{array}\right),\left(\begin{array}{ll}
0 & 0 \\
x & 0
\end{array}\right)\right]=\left(\begin{array}{cc}
x & 0 \\
0 & x
\end{array}\right) \in \mathfrak{p}_{0}
$$

Therefore $\mathbb{D}$ acts on an element in $V(\lambda)$ as

$$
\begin{aligned}
& \mathbb{D}\left(x_{1} \wedge \ldots \wedge x_{r} \otimes \varphi\right) \\
& =\left[\mathbb{D}, x_{1}\right] x_{2} \wedge \ldots \wedge x_{r} \otimes \varphi-x_{1} \wedge\left[\mathbb{D}, x_{2}\right] x_{3} \wedge \ldots \wedge x_{r} \otimes \varphi \ldots \\
& +(-1)^{r+1} x_{1} \wedge \ldots\left[\mathbb{D}, x_{r}\right] \otimes \varphi \\
& =\left(\begin{array}{cc}
x_{1} & 0 \\
0 & x_{1}
\end{array}\right)\left(x_{2} \wedge \ldots \wedge x_{r} \otimes \varphi\right)-x_{1} \wedge\left(\begin{array}{cc}
x_{2} & 0 \\
0 & x_{2}
\end{array}\right)\left(x_{3} \wedge \ldots \wedge x_{r} \otimes \varphi\right)+\ldots \\
& +(-1)^{r+1} x_{1} \wedge \ldots \wedge x_{r-1} \otimes\left(\begin{array}{cc}
x_{r} & 0 \\
0 & x_{r}
\end{array}\right)(\varphi)
\end{aligned}
$$

where the derivations $\left[\mathbb{D}, x_{\nu}\right] \in \mathfrak{p}_{0}$ act on all terms to the right. The $\theta_{1}$-term arises from the action of $\left[\mathbb{D}, x_{\nu}\right]$ on the last term $\varphi$ to the right, the remaining terms lead to a sum with the $\sum_{\mu<\nu}$-condition defining the $\theta_{0}$-term.

Viewing $\theta:=x_{1} \wedge \cdots \wedge x_{r}$ as an element in the universal enveloping algebra $U\left(\mathfrak{p}_{-}\right)$of $\mathfrak{p}_{-}$, the super commutator $[\mathbb{D}, \theta]$ in the universal enveloping algebra $U(\mathfrak{p})$ of $\mathfrak{p}$ is $[\mathbb{D}, \theta]=\theta_{0}+\theta_{1}$ with $\theta_{1}$ in the universal enveloping algebra $U\left(\mathfrak{p}_{-} \oplus \mathfrak{p}_{0}\right)$ of $\mathfrak{p}_{-} \oplus \mathfrak{p}_{0}$ and $\theta_{0}$ in the universal enveloping algebra $U\left(\mathfrak{p}_{-}\right)$of 
$\mathfrak{p}_{-}$as defined above, but viewed as element in the universal enveloping algebra of $\mathfrak{p}$. Furthermore $\theta_{1}$ annihilates $H$-invariant vectors in any $\mathfrak{p}$-module. Stated in this form, the assertion obviously holds for arbitrary elements $\theta$ in the universal enveloping algebra of $\mathfrak{p}_{-}$.

$H^{\bullet}(V(\lambda))$ and the theorem of Hopf. Lemma 26.1 identifies $H_{D S_{n}}^{\bullet}(V(\lambda))$ with the Lie algebra homology ring $H_{\bullet}(\mathfrak{p}, \operatorname{End}(\rho))$. We recall some facts about Lie algebra (co)homology. Note that $H_{\bullet}(\mathfrak{g})=H^{\bullet}(\mathfrak{g})$.

Let $\mathfrak{g}$ be a reductive Lie algebra and $\Lambda^{\bullet}(\mathfrak{g})^{\mathfrak{g}}$ the space of invariants under the adjoint action of $\mathfrak{g}$. It has the structure of a graded super Hopf algebra. Let $P(\mathfrak{g})$ denote the space of primitive elements, i.e.

$$
P(\mathfrak{g})=\left\{x \in \Lambda^{\bullet}(\mathfrak{g})^{\mathfrak{g}} \mid \Delta(x)=x \otimes 1+1 \otimes x\right\}
$$

where $\Delta$ denotes the comultiplication. Define a grading on $P(\mathfrak{g})$ by requiring that the inclusion $P(\mathfrak{g}) \rightarrow \Lambda^{\bullet}(P(\mathfrak{g}))$ preserves degrees.

Theorem 26.2. [Mei13, Theorem 10.2, Corollary 10.2, Corollary 10.3] (Hopf-Koszul-Samelson)

(1) The inclusion of $P(\mathfrak{g})$ in $\Lambda^{\bullet}(\mathfrak{g})^{\mathfrak{g}}$ extends to an isomorphism of graded super Hopf algebras $\Lambda^{\bullet}(P(\mathfrak{g})) \cong \Lambda^{\bullet}(\mathfrak{g})^{\mathfrak{g}}$.

(2) There is an isomorphism $H^{\bullet}(\mathfrak{g}) \cong \Lambda^{\bullet}(P(\mathfrak{g}))$ of graded super Hopf algebras, i.e. the cohomology ring is an exterior algebra over the primitive elements. In particular the elements in $\Lambda^{\bullet}(P(\mathfrak{g}))$ are closed.

(3) The space of primitive elements has dimension rank(g). For $\mathfrak{g l}(n)$ the basis elements $f_{1}, f_{3}, . ., f_{2 n-1} \in P(\mathfrak{g})$ have degree $1,3, . ., 2 n-1$.

We now apply this theorem for the Lie algebra $\mathfrak{g}$ of $H$ and the $H$-invariant ring $I$ in the universal enveloping algebra of $\mathfrak{p}_{-}$using the following identifications $I \cong \Lambda^{\bullet}\left(\mathfrak{p}_{-}\right)^{H} \cong \Lambda^{\bullet}\left(\mathfrak{p}_{0}\right)^{H} \cong \Lambda^{\bullet}(\mathfrak{g})^{\mathfrak{g}} \cong V(\mathbf{1})^{H}$ for the invariant ring

$$
I:=U\left(\mathfrak{p}_{-}\right)^{H}
$$

From theorem 26.2 we obtain the following corollary.

Corollary 26.3. The cohomology $H_{D S_{n}}^{\bullet}(V(1))$ is isomorphic to $I \cong V(\mathbf{1})^{H}$ and I has the structure of a supercommutative polynomial ring $\mathbb{C}\left\{f_{1}, . ., f_{2 n-1}\right\}$ generated by elements $f_{\nu}$ in the degrees $1-2 \nu$ for $\nu=1, . ., n$. In particular

$$
\omega_{D S_{n}}(t)=\prod_{\nu=1}^{n}\left(1+t^{1-2 \nu}\right) .
$$

Lemma 26.4. For any p-module $V$, the cohomology group $H_{D S_{n}}^{\bullet}\left(V^{H}\right)$ is a graded I-module.

Proof. By theorem 26.2 we have $\mathbb{D}(v)=\theta_{0}(v)+\theta_{1}(v)=0$ for every element $v \in V(1)^{H}$. Since $\theta_{1}(v)=0$ holds for $H$-invariant vectors, we get 
$\theta_{0}(v)=0$ and hence $\theta_{0}=0$ holds in $U\left(\mathfrak{p}_{-}\right)$. This implies $[\mathbb{D}, \theta]=\theta_{1}$ for all $\theta \in U\left(\mathfrak{p}_{-}\right)^{H} \cong \Lambda^{\bullet}\left(\mathfrak{p}_{-}\right)^{H}=I$. For any $P \in I$, hence $[\mathbb{D}, P]=P_{1} \in U(\mathfrak{p})$ annihilates $H$-invariant vectors. For any finite dimensional algebraic $\mathfrak{p}$-module $V, V$ in particular is an $U\left(\mathfrak{p}_{-}\right)$-module and the subspace $V^{H}$ obviously is an $I$-module. Since $\mathbb{D}$ commutes with $H$, we obtain a linear map $\mathbb{D}: V^{H} \rightarrow$ $V^{H}$. For $P \in I$ and $v \in V^{H}$ the formulas $\mathbb{D}(P v)=[\mathbb{D}, P] v+P \mathbb{D}(v)$ and $[\mathbb{D}, P]=P_{1}$ and $P_{1} v=0$ imply $\mathbb{D}(P v)=P \mathbb{D}(v)$ for all $v \in V^{H}$. Hence the subspace of $\mathbb{D}$-coboundaries resp. of $\mathbb{D}$-closed elements in $V^{H}$ are both $I$-modules.

Lemma 26.5. For finite-dimensional $\mathfrak{g l}(n \mid n)$-modules $M$ the following holds: $H_{D S_{n}}^{\bullet}(M) \cong H_{D S_{n}}^{\bullet}\left(M^{H}\right)$.

Proof. $H$ commutes with $\mathbb{D}$ and operates therefore on the cohomology $H_{D S_{n}}^{\bullet}(M)$. Since $H$ is reductive, a finite-dimensional representation of $H$ is trivial if and only if its restriction to a Cartan subgroup is trivial. We therefore show that the diagonal torus $T \subset H$ acts trivially on the cohomology. By the Leray spectral sequence

$$
D S_{n, n-1} \circ D S_{n-1, n-2} \circ \ldots \circ D S_{1,0} \Longrightarrow D S_{n, 0}=D S_{n} .
$$

By section $5 D S_{n, n-1}$ is invariant under

$$
H_{n, n-1}=\left(\begin{array}{cccc}
0 & & & \\
& \cdots & & \\
& & 0 & \\
& & & 1
\end{array}\right),
$$

$D S_{n-1, n-2}$ is invariant under

$$
H_{n-1, n-2}=\left(\begin{array}{cccc}
0 & & & \\
& \cdots & & \\
& & 1 & \\
& & & 0
\end{array}\right)
$$

and so on. Hence $H_{D S_{n}}^{\nu}(M)$ has a filtration which is respected by $T$ such that $T$ acts trivially on the graded pieces. Since $T$ acts in a semisimple way, this implies that the operation of $T$, and therefore of $H$, is trivial.

Proposition 26.6. For $M \in \mathcal{R}_{n}$ the cohomology $H_{D S_{n}}^{\bullet}(M)$ is a graded $I$-module for the graded polynomial ring $I$. For morphisms $f: M \rightarrow M^{\prime}$ in $\mathcal{R}_{n}$ the induced map $H_{D S_{n}}^{\bullet}(M) \rightarrow H_{D S_{n}}^{\bullet}\left(M^{\prime}\right)$ is graded I-linear.

Proof. This follows from the lemmas 26.4 and 26.5 .

Lemma 26.7. Let $\rho$ be an irreducible representation of $G l(n)$. Then the map

$$
\varphi:\left.\left.V(\mathbf{1})\right|_{\mathfrak{p}} \rightarrow V\left(\rho^{\vee} \otimes \rho\right)\right|_{\mathfrak{p}}, \quad v \otimes 1 \mapsto v \otimes i d_{\rho}
$$

is a $\mathfrak{p}$-linear inclusion. 
Proof. We have $x_{\nu}(\varphi)=0$ for all $\nu$ if $\varphi \in \operatorname{End}_{H}(\rho)=\mathbb{C} i d_{\rho}$.

Remark. Every maximal atypical $\mathfrak{g}$-module $V$, when restricted to $\mathfrak{g}_{0}$, has the form $\left.V\right|_{\mathfrak{g}_{0}}=\bigoplus \rho_{\nu} \otimes \rho_{\mu}$ with $\operatorname{deg}\left(\rho_{\nu}\right)=\operatorname{deg}\left(\rho_{\mu}\right)$. This degree makes $V$ into a graded $\mathfrak{p}$-module. For $V(\mathbf{1})$ we obtain the degree defined previously. For $V\left(\rho^{\vee} \otimes \rho\right), i d_{\rho}$ has degree $\operatorname{deg}(\rho)$. Therefore $\varphi$ shifts the degrees by $\operatorname{deg}(\rho)$. The degree $\operatorname{deg}(\rho)$ coincides with $p(\lambda)$ for $\rho$ with highest weight $\lambda=\left(\lambda_{1}, \ldots, \lambda_{n}\right)$.

Lemma 26.8. The induced morphism

$$
H_{D S_{n}}^{\bullet}(V(\mathbf{1})) \stackrel{H_{\dot{D S}_{n}}(\varphi)}{\longrightarrow} H_{D S_{n}}^{\bullet+\operatorname{deg}(\rho)}\left(V\left(\rho^{\vee} \otimes \rho\right)\right)
$$

is a graded isomorphism on the cohomology. Hence $H_{D S_{n}}^{\bullet+\operatorname{deg}(\rho)}\left(V\left(\rho^{\vee} \otimes \rho\right)\right)$ is the free I-module of rank one generated by the top cohomology.

Proof. As a p-module

$$
\left.V\left(\rho^{\vee} \otimes \rho\right)\right|_{\mathfrak{p}} \cong \varphi(V(\mathbf{1})) \oplus\left(\Lambda^{\bullet}\left(\mathfrak{p}_{-}\right) \otimes E n d^{0}(\rho)\right)
$$

where

$$
\operatorname{End}^{0}(\rho)=\{\phi \in \operatorname{End}(\rho) \mid \operatorname{Tr}(\phi)=0\}
$$

Since $D \in \mathfrak{p}$, we obtain

$$
H_{D S_{n}}^{\bullet}\left(V\left(\rho^{\vee} \otimes \rho\right)\right)=H_{D S_{n}}^{\bullet}(\varphi(V(\mathbf{1}))) \oplus H_{D S_{n}}^{\bullet}\left(\Lambda^{\bullet}\left(\mathfrak{p}_{-}\right) \otimes E n d^{0}(\rho)\right) .
$$

By [HS53] the Lie algebra cohomology for reductive $H$ with coefficients in a representation $W$ is trivial except for the trivial representation

$$
H^{\bullet}(\operatorname{Lie}(H), W) \cong H^{\bullet}(\operatorname{Lie}(H), \mathbf{1}) \otimes W^{H} .
$$

Since the Lie algebra cohomology is dual to the homology, this shows

$$
H_{D S_{n}}^{\bullet}\left(\Lambda^{\bullet}\left(\mathfrak{p}_{-}\right) \otimes E n d^{0}(\rho)\right)=0
$$

Since $\delta=a d_{\mathbb{D}}$ commutes with $\varphi$, we get

$$
H_{D S_{n}}^{\bullet}(V(\mathbf{1})) \underset{H_{D S_{n}}^{\bullet}(\varphi)}{\cong} H_{D S_{n}}^{\bullet}\left(\Lambda^{\bullet}\left(\mathfrak{p}_{-}\right) \otimes i d_{\rho}\right)
$$

up to the degree shift with $\operatorname{deg}(\rho)$.

Corollary 26.9. For the Hilbert polynomial of $V(\lambda)$ relative to $D$ we obtain

$$
\omega_{D S_{n}}(V(\lambda), t)=t^{p(\lambda)} \cdot \omega_{D S_{n}}(V(\mathbf{1}), t)=t^{p(\lambda)} \cdot \prod_{\nu=1}^{n}\left(1+t^{1-2 \nu}\right) .
$$

Theorem 26.10. Let $L(\lambda)$ be an irreducible and maximal atypical representation and pr: $V(\lambda) \rightarrow L(\lambda)$ be a projection onto the top. Then the induced homomorphism

$$
H_{D S_{n}}^{\nu}(p r): H_{D S_{n}}^{\nu}(V(\lambda)) \rightarrow H_{D S_{n}}^{\nu}(L(\lambda))
$$


is zero in degrees $\nu<p(\lambda)$ and an isomorphism for $\nu=p(\lambda)$.

Proof. As a graded $I$-module $H_{D S_{n}}^{\nu}\left(V\left(\rho^{\vee} \otimes \rho\right)\right)$ is the free $I$-module generated by the cohomology in the top degree. To prove our claim it suffices that the primitive elements $f_{1}, f_{3}, \ldots, f_{2 n}-1 \in I$ act trivially on $H_{D S_{n}}^{\nu}\left(L\left(\rho^{\vee} \otimes \rho\right)\right)$. This follows from the discussion in section 25, lemma 25.2, which shows for $\nu=1, \ldots, n$

$$
H_{D S_{n}}^{\operatorname{deg}(\rho)-2 \nu+1}\left(L\left(\rho^{\vee} \otimes \rho\right)\right)=0
$$

\section{PRimitive Elements of $H_{D S_{n}}^{\bullet}(V(\mathbf{1}))$}

We will now describe the primitive elements of $H_{D S_{n}}^{\bullet}(V(\mathbf{1}))$ in terms of the representation theory of the superlinear group $G l(n \mid n)$. The radical filtration on $V(\mathbf{1})$ defines a decreasing filtration $F_{i}$ of $V(\mathbf{1})$. The $H$-invariants $F_{i}^{H}$ coincide with the powers $\left(I^{+}\right)^{i}$ of the augmentation ideal $I^{+}$of the invariant ring $I=V(\mathbf{1})^{H}$. In this way monomials of degree $i$ in the primitive generators $f_{1}, \ldots, f_{2 n-1}$ can be identified with the generators of the cohomology $H^{\bullet}\left(F_{i}^{H} / F_{i+1}^{H}\right)$.

The Murnagan-Nakayama rule. Let $\lambda=\left(\lambda_{1}, \ldots, \lambda_{r}\right)$ with $\lambda_{1} \geq \lambda_{2} \geq \cdots \geq \lambda_{r}$ be a partion of degree $n=\operatorname{deg}(\lambda)$. For partitions $\nu$ and $\mu$ of $m$ and $n-m$ let $c_{\mu \nu}^{\lambda}$ denote the Littlewood-Richardson coefficient. Assume that $\nu$ is a hook, i.e a partition of type $\nu_{1}=r, \nu_{2}=\cdots=\nu_{m-r+1}=1$ and $\nu_{i}=0$ for $i>m-r+1$. Recall that a hook is a special case of a rim hook (also called skew hook). We say $\nu$ is a symmetric hook if $m=2 r-1$. According to [Sa01, Section 4.10] we have

Proposition 27.1. Suppose $\nu$ is a hook. Then $c_{\mu \nu}^{\lambda}=0$ unless the Young diagram of $\mu$ is contained in the Young diagram of $\lambda$ and the complement $\lambda / \mu$ is a union of $k$ edgewise connected rim hooks. If this is the case, then

$$
c_{\mu \nu}^{\lambda}=\left(\begin{array}{l}
k-1 \\
c-r
\end{array}\right)
$$

where $r=\nu_{1}$ and $c$ is the number of rows spread by the rim hooks contained in $\lambda / \mu$.

We remark that in [Sa01] $c$ denotes the number of columns instead of rows, since Young diagrams in [Sa01] are written top down instead of being written from left to right, as with our conventions.

Corollary 27.2. Suppose $\lambda$ and $\nu$ are symmetric hooks. Then $c_{\mu \nu}^{\lambda}=0$ unless $\mu=\nu$ or $\nu=0$.

Proof. Suppose $c_{\mu \nu}^{\lambda} \neq 0$. By the proposition the edgewise connected components of $\lambda / \mu$ are rim hooks, hence $\#(\lambda / \mu) \leq 2$. Since $\operatorname{deg}(\nu)$ is 
odd for symmetric hooks and $\#(\lambda / \mu)=\operatorname{deg}(\nu)$, we may assume without restriction of generality that $\#(\lambda / \mu)=1$. But this gives a contradiction since $\operatorname{deg}(\lambda)-\operatorname{deg}(\mu)=1$ would be the difference of two odd numbers.

Let $\rho^{\vee}$ denote the dual representation of $\rho$. Suppose $\lambda$ is a partition of $n$ and $\lambda^{*}$ is the dual partition of $n$, then define $\left(\rho_{\lambda}\right)^{*}:=\rho_{\lambda^{*}}$ for the representations $\rho=\rho_{\lambda}$ of $G L(n)$ with highest weight $\lambda$.

Corollary 27.3. Suppose that $\nu$ is a symmetric hook of degree $2 r-1$ and suppose $k=1$ (in the notation of proposition 27.1). Suppose the rim hook $\lambda / \mu$ reaches from $\left(i, \lambda_{i}\right)$ to $\left(j, \lambda_{j}\right)$ where $i>j$. Then $c_{\mu \nu}^{\lambda}=0$ hold unless $\lambda_{i}-\lambda_{j}=i-j=r$.

Proof. Since $k=1,\left(\begin{array}{l}k-1 \\ c-r\end{array}\right) \neq 0$ if and only if $\lambda_{i}-\lambda_{j}=c=r$. Since $\nu$ is a rim hook, we have $2 r-1=\operatorname{deg}(\nu)=\left(\lambda_{i}-\lambda_{j}\right)+(j-i)-1$. Hence $\lambda_{i}-\lambda_{j}=r$ implies $j-i=r$.

The Lie superalgebra $\mathfrak{g l}(n \mid n)$ and primitive elements of $\mathfrak{g l}(n)$. The following proposition is a well-known consequence of the dual Cauchy identity.

Proposition 27.4. [BSch17, Theorem B.17] The space of matrices $M_{n n}(k)$ is a $G l(n, k) \times G l(n, k)$-module in a natural way by left and right multiplication, hence also the Graßmann algebra $\Lambda:=\Lambda^{\bullet}\left(M_{n}(k)\right)$. As a representation of $G l(n, k) \times G l(n, k)$ we have

$$
\Lambda^{\bullet}\left(M_{n n}(k)\right) \cong \bigoplus_{\rho} \rho^{\vee} \otimes \rho^{*}
$$

where $\rho=\rho_{\lambda}$ runs over all partitions in

$$
P(n, n)=\left\{\lambda \in \mathbf{Z}^{n} \mid n \geq \lambda_{1} \geq \lambda_{2} \geq \ldots \geq \lambda_{n} \geq 0\right\} .
$$

Warning: The degree $\operatorname{deg}\left(\rho^{\vee}\right)$ is the the negative of the degree in the Graßmann algebra $\Lambda$ !

Corollary 27.5. Let $H=G L(n, k)$ be embedded diagonally. Then

$$
I:=\Lambda^{\bullet}\left(M_{n n}(k)\right)^{H} \cong \bigoplus_{\rho}\left(\rho^{\vee} \otimes \rho^{*}\right)^{H},
$$

and $\left(\rho^{\vee} \otimes \rho^{*}\right)^{H} \neq 0$ if and only if $\rho=\rho_{\lambda}$ for a symmetric Young diagram $\lambda=\lambda^{*}$. There exist $2^{n}$ symmetric Young diagrams with $\lambda=\left(\lambda_{1}, \ldots, \lambda_{n}\right)$ and $\lambda_{1} \leq n$.

The space $I$ is an algebra with respect to the wedge product. The subspace $I^{+} \subseteq I$ of elements of degree $\geq 1$ is an ideal (the augmentation ideal).

Proposition 27.6. [Mei13, Proposition 10.11] $I^{+}$decomposes as

$$
I^{+}=P(H) \oplus\left(I^{+}\right)^{2} \text {. }
$$


Corollary 27.7. With summation over all $\rho=\rho_{\lambda}$ for symmetric hook diagrams $\lambda$ of degrees deg $(\lambda)=1,3,5, \ldots, 2 n-1$ the space of primitive elements is

$$
P(H)=\bigoplus_{\rho}\left(\rho^{\vee} \otimes \rho^{*}\right)^{H} .
$$

Proof. This follows from the fact that for hook diagrams $\lambda$ the space $\rho_{\lambda}$ cannot be a constituent of $\rho_{\mu} \otimes \rho_{\nu}$ for $\mu=\mu^{*}$ and $\nu=\nu^{*}$ where $\left(\rho_{\mu}^{\vee} \otimes \rho_{\mu}^{*}\right)^{H}$ and $\left(\rho_{\nu}^{\vee} \otimes \rho_{\nu}^{*}\right)^{H}$ are constituents of $I^{+}$. Hence $\left(\rho_{\lambda}^{\vee} \otimes \rho_{\lambda}^{*}\right)$ cannot be contained in $\left(I^{+}\right)^{2}$.

The index. The selftransposed weights $\lambda_{(i)}=(i, . ., i, 0, . ., 0)$ for $i=0, . ., n$ in $P(n, n)$ are called the basic selftransposed weights. The index ind $(\lambda)$ of a selftransposed $\lambda$ in $P(n, n)$ is the maximal index $i$ of a basic selftransposed $\lambda_{i}$ whose Young diagram is contained in the Young diagram of $\lambda$. The index of $\lambda$ is the unique $i$ between 1 and $n$ such that $\lambda_{i} \geq i$ and $\lambda_{i+1} \leq i$. We denote by $P_{i}(n, n)$ the set of all weights in $P(n, n)$ with index $i$.

Proposition 27.8. Using that $\Lambda \cong V(\mathbf{1})$, the canonical filtration defined by the radical filtration of $V(\mathbf{1})$ in the category of $\mathfrak{g l}(n \mid n)$-modules gives a filtration $F_{i}$ on $\Lambda$ such that

$$
F_{i}=\bigoplus_{\rho}\left(\rho^{\vee} \otimes \rho^{*}\right)
$$

for all $\rho=\rho_{\lambda}$ running over all partitions $\lambda$ containing the partition $\left(i^{i}\right)$ of degree $i^{2}$.

Before the proof we recall that $V(\mathbf{1})$ has a decreasing filtration (the radical filtration) of $G l(n \mid n)$-subrepresentations with $n+1$ irreducible graded pieces $L_{i}$ such that $L_{0}=k$ is the maximal irreducible quotient representation. The highest weights of the $L_{i}$ can be computed from [BS11, Theorem 5.2] to be the duals

$$
\lambda_{(i)}^{\vee}=(0, \cdots, 0,-i, \ldots,-i) \quad, \quad \text { for } i=0, \ldots, n
$$

of the basic selftransposed weights $\lambda_{i}$ in $P(n, n)$.

Proof. We need to show that the representation $L_{i}$, considered as a representation of $G \subset G l(n \mid n)$, decomposes into a direct sum over the duals of all irreducible representations $\rho(\lambda) \otimes \rho\left(\lambda^{*}\right)$ for which

$$
\lambda \in P_{i}(n, n) .
$$

Consider the decomposition of $L_{i}^{\vee}$ under $G=G l(n) \times G l(n)$. Let $\lambda=$ $\left(\lambda_{1}, . ., \lambda_{n}\right)$ be a corresponding highest weight of $G$ in $L_{i}$. We then claim $\operatorname{ind}(\lambda)=i$. Obviously

$$
\lambda \geq \lambda_{(i)}=(i, \ldots, i, 0, \ldots, 0) .
$$


On the other hand we have

$$
V(\mathbf{1})^{\vee} \cong\left(\operatorname{det}^{n} \otimes d e t^{n}\right) \otimes V(\mathbf{1})
$$

since the dual of a Kac module is a Kac module. Hence, since the order of the socle layers in the dual Kac module is reversed and since the Loewy length of $V(\mathbf{1})$ is $n+1$ [BS11, Theorem 5.2], this implies

$$
L_{i}^{\vee} \cong\left(\operatorname{det}^{n} \otimes d e t^{n}\right) \otimes L_{n-i}
$$

This in turn implies

$$
\lambda \leq(n, \ldots, n)+\lambda_{(n-i)}^{\vee}=(n, \ldots, n, i, \ldots, i)
$$

with $i$ copies of $n$ and $n-i$ copies of $i$. Both estimates together force $\lambda_{i} \geq i$ and $\lambda_{i+1} \leq i$, hence $i n d(\lambda)=i$. This proves our claim. Since any $\lambda$ in $P(n, n)$ appears in one of the $L_{i}^{\vee}, L_{i}^{\vee}$ then consists precisely of the $G$-constituents $\rho(\lambda) \otimes \rho\left(\lambda^{*}\right)$ for $\lambda$ in $P_{i}(n, n)$.

Corollary 27.9. $F_{i}^{H}=\left(I^{+}\right)^{i}$, hence we can identify monomials of degree $i$ in the primitive generators $f_{1}, \ldots, f_{2 n-1}$ with the generators of the cohomology $H^{\bullet}\left(F_{i}^{H} / F_{i+1}^{H}\right)$.

We introduce the notation $\operatorname{Prim}_{i} \subseteq I^{+}$for the space that is spanned by monomials in the primitive elements $f_{2 \nu-1}$ with exactly $i$ factors. In this notation Prim $_{1}=P(H)$.

Proof. By corollary 27.7 Prim $_{1}$ is a complement to $F_{2}^{H}$. We now show $\operatorname{Prim}_{i} \cap F_{i+1}^{H}=0$ by induction on $i$. Using the induction assumption and $\operatorname{Prim}_{i}=\operatorname{Prim}_{i-1} \cdot \operatorname{Prim}_{1}$, the space Prim $i$ only gives rise to Young diagrams $\lambda$ that occur in the tensor product of some $\mu \in P_{j}(n, n)$ for $j<i$ and a symmetric hook $\nu \in P_{1}(n, n)$. By proposition 27.1, $\mu$ is obtained from $\lambda$ by removing a (possibly disconnected) rim hook. If $\lambda \in P_{k}(n, n)$, this implies $j=k$ or $j=k-1$ and hence $k \leq j+1 \leq i$. This proves $\operatorname{Prim}_{i} \cap F_{i+1}^{H}=0$ since all selftransposed weights $\lambda$ in $F_{i+1}^{H}$ are contained in $P_{k}(n, n)$ for $k \geq i+1$.

This implies $\operatorname{Prim}_{\nu} \cap F_{j}^{H}=0$ for $\nu<j$. Since $I^{+}$is the direct sum of $\bigoplus_{\nu=0}^{i} \operatorname{Prim}_{\nu}$ and $\left(I^{+}\right)^{i+1}$, this implies that $F_{i+1}^{H}$ is in the complement of $\bigoplus_{\nu=0}^{i} \operatorname{Prim}_{\nu}$ and therefore $F_{i+1}^{H} \subseteq\left(I^{+}\right)^{i+1}$. There are $\left(\begin{array}{c}n \\ i\end{array}\right)$ selftransposed weights $\lambda \in P_{i}(n, n)$ and all of them occur in $V(\mathbf{1})$ with multiplicity one. On the other hand, the space $\operatorname{Prim}_{i} \subseteq I^{+}$that is spanned by monomials in the primitive elements $f_{2 \nu-1}$ with exactly $i$ factors also has dimension $\left(\begin{array}{c}n \\ i\end{array}\right)$. Since the dimensions agree, this implies $\left(I^{+}\right)^{i+1}=F_{i+1}^{H}$ and $\operatorname{Prim}_{i}$ is therefore represented by $\left(F_{i} / F_{i+1}\right)^{H} \cong H_{D S_{n}}^{\bullet}\left(F_{i} / F_{i+1}\right)$. 


\section{KAC MODULE OF 1}

Overview. We now study the effect of $D S$ on indecomposable modules in the remaining sections $28-31$. The easiest examples are perhaps the extensions of two irreducible modules, and we focus here on the case of extensions of the trivial representation 1 by another irreducible module. Our main result in these sections is corollary 31.5 , saying that a representation $Z$, such that the projection onto its cosocle 1 induces a surjection $\omega: \omega(Z) \rightarrow \omega(\mathbf{1})$, is equal to the trivial representation. Such a representation $Z$ contains extensions of the trivial representation 1 with other irreducible representations. We show in the resumé of section 29 that if $Z$ is not irreducible, we obtain extensions $V$ of 1 by an irreducible representation $S$ such that the induced morphism $\omega(V) \rightarrow \omega(\mathbf{1})$ is surjective. Since the dimension of $\operatorname{Ext}^{1}(S, \mathbf{1})$ is at most one-dimensional, any two such extensions are isomorphic. Hence we can study them by realizing them as quotients of modules whose cohomology is sufficiently understood. A typical example occurs in the current section 28: The Kac module $V(\mathbf{1})$ of 1 contains an extension of 1 with the irreducible representation $[0, \ldots, 0,-1]$; and by considering the cohomology of the Kac module we are able to compute the cohomology of this extension and its dual in lemma 28.2 and lemma 28.4. We also show in corollary 28.6 that $\omega^{0}(V)=0$ for the extension $V$ between 1 and $\operatorname{Ber} \otimes S^{n-1}$. The other $n$ nontrivial extensions of 1 (listed in lemma 31.2) are studied in section 31 . We realize these extensions as a quotient of the mixed tensor $R\left(n^{n}\right)$ studied in section 30. The key proposition 31.1 shows that for any of our nontrivial extensions $V$ the zero degree part $\omega^{o}$ of the induced map $\omega\left(q_{V}\right): \omega(V) \rightarrow \omega(\mathbf{1})$ vanishes, a contradiction our analysis in the resumé of section 29 , hence $Z \simeq \mathbf{1}$.

The constituents of the Kac module $V(\mathbf{1}) \in \mathcal{R}_{n}$ are [BS11], thm. 5.2,

$$
L_{a}=\operatorname{Ber}^{-a} \otimes[a, \ldots, a, 0, \ldots, 0] \text { for } a=0, \ldots, n,
$$

where the last entry of $a$ is at the position $i=n-a$. Therefore $B e r^{a} \otimes L_{a}$ is basic and therefore has cohomology concentrated in degree zero, hence the cohomology of $L_{a}$ is concentrated in degree $-a$ and

$$
H^{-a}\left(L_{a}\right) \cong I_{a} \oplus I_{a-1} \quad, \quad a=0,1, \ldots, n
$$

where $I_{-1}:=I_{n}:=0$ and

$$
I_{a}:=\operatorname{Ber}^{-a-1} \otimes[a+1, \ldots, a+1,0, \ldots, 0]
$$

(with $n-a-1$ entries $a+1$ and $a$ entries 0 ). Notice $I_{1}^{\vee} \cong \operatorname{Ber} \otimes S^{n-1}$ and $I_{0}=1, I_{1}=[0, . ., 0,-2], \ldots, I_{n-1}=B e r^{-n}$. For the cyclic quotient $Q_{a}$ of $V(\mathbf{1})$ with socle $L_{a}$ this implies inductively 
Lemma 28.1. The natural quotient map $Q_{a} \rightarrow 1$ induces an isomorphism $H^{0}\left(Q_{a}\right) \cong H^{0}(\mathbf{1}) \cong \mathbf{1}$ and

$$
H^{-\nu}\left(Q_{a}\right)=\left\{\begin{array}{ll}
I_{\nu} \oplus I_{\nu-1} & \nu=0, \ldots, a, \\
0 & \text { otherwise. }
\end{array} .\right.
$$

Notice $Q_{a}=V(\mathbf{1})$ for $a=n$ and $Q_{a}=\mathbf{1}$ for $a=0$. Similar as in the proof of the last lemma, for $\left.K_{a}=\operatorname{Ker}\left(V(\mathbf{1}) \rightarrow Q_{a}\right)\right)$ and $a \leq n-1$ we obtain exact sequences

$$
0 \rightarrow H^{\bullet}\left(K_{a}\right) \rightarrow H^{\bullet}(V(\mathbf{1})) \rightarrow H^{\bullet}\left(Q_{a}\right) \rightarrow 0
$$

Indeed, the cohomology of $H^{\bullet}\left(K_{a}\right)$ is concentrated in degrees $\leq-a-1$, whereas the cohomology of $H^{\bullet}\left(Q_{a}\right)$ is concentrated in degrees $\geq-a$. We can view these as short exact sequences of homology complexes

$$
0 \rightarrow\left(H^{\bullet}\left(K_{a}\right), \bar{\partial}\right) \rightarrow\left(H^{\bullet}(V(\mathbf{1})), \bar{\partial}\right) \rightarrow\left(H^{\bullet}\left(Q_{a}\right), \bar{\partial}\right) \rightarrow 0
$$

The long exact homology sequence for the $H_{\bar{\partial}}$-homology together with $H_{\bar{\partial}}\left(H^{\nu}(V)\right)=H_{D}^{\nu}(V)$ (lemma 6.3) implies

$$
H_{D}^{-\nu}\left(K_{a}\right) \longrightarrow H_{D}^{-\nu}(V(\mathbf{1})) \longrightarrow H_{D}^{-\nu}\left(Q_{a}\right) \stackrel{\delta}{\longrightarrow} H_{D}^{-\nu-1}\left(K_{a}\right) \longrightarrow H_{D}^{-\nu-1}(V(\mathbf{1}))
$$

and $H_{D}^{\nu}(V(\mathbf{1}))=0$ for all $\nu$ hence gives $H_{D}^{\nu}\left(Q_{a}\right) \cong H_{D}^{\nu-1}\left(K_{a}\right)$. Now $H_{D}^{\nu}\left(Q_{a}\right)$ vanishes unless $\nu \geq-a$ by lemma 28.1. The right hand side $H^{\nu-1}\left(K_{a}\right)$ is concentrated in degrees $\nu \leq-a$. Hence the long exact homology sequence has at most one nonvanishing connecting morphism $\delta$, namely $\delta: H_{D}^{-a}\left(Q_{a}\right) \rightarrow H_{D}^{-a-1}\left(K_{a}\right)$ in degree $-a$. Hence $H_{D}^{\nu}\left(Q_{a}\right)=0$ for $\nu \neq-a$. Since there is a unique common irreducible module $I_{a}$ in the cohomology $H^{-1-a}\left(K_{a}\right)$ and $H^{-a}\left(Q_{a}\right)$ such that $d\left(Q_{a}\right)= \pm I_{a}$, we conclude

Lemma 28.2. For $0 \leq a \leq n-1$ we get

$$
H_{D}^{\nu}\left(Q_{a}\right)= \begin{cases}I_{a} & \nu=-a \\ 0 & \text { otherwise }\end{cases}
$$

Remark. This result shows that for the $H_{D}^{\nu}$-cohomology there are do not exist long exact sequences attached to short exacts sequences in $\mathcal{R}_{n}$. If these would exist, then $Q_{1} / L_{1} \cong 1$ would imply $H_{D}^{-1}\left(L_{1}\right) \cong H_{D}^{-1}\left(Q_{1}\right)$, in contrast to $H_{D}^{-1}\left(L_{1}\right) \cong I_{1} \oplus \mathbf{1}$ and $H_{D}^{-1}\left(Q_{1}\right) \cong I_{1}$.

Corollary 28.3. $H_{D}^{0}(V)=0$ for $V=Q_{a}$ and $\left(Q_{a}^{*}\right)^{\vee}$ for $1 \leq a \leq n-1$.

Now we analyse in the case $a=1$ the nontrivial extension

$$
0 \rightarrow[0, \ldots, 0,-1] \rightarrow Q_{1} \rightarrow \mathbf{1} \rightarrow 0
$$


Since $L_{1}^{\vee} \cong[0, \ldots, 0,-1]^{\vee} \cong \operatorname{Ber} \otimes S^{n-1}$, also $V=\left(Q_{1}^{*}\right)^{\vee}$ defines a nontrivial extension

$$
0 \rightarrow \operatorname{Ber} \otimes S^{n-1} \rightarrow V \rightarrow \mathbf{1} \rightarrow 0
$$

Lemma 28.4. $V=\left(Q_{1}^{*}\right)^{\vee}$ defines a nontrivial extension between 1 and $\operatorname{Ber} \otimes S^{n-1}$ in $\mathcal{R}_{n}$ such that in $\mathcal{R}_{n-1}$ the following holds

$$
H_{D}^{\nu}(V) \cong H^{\nu}(V)= \begin{cases}B \operatorname{er} \otimes S^{n-1} & \nu=1, \\ 0 & \text { otherwise. }\end{cases}
$$

Proof. The statement about $H_{D}^{\nu}(V)$ follows immediately from lemma 28.2. We now calculate $H^{\nu}(V)$. Since the cohomology of the anti-Kac module $\left(V(\mathbf{1})^{*}\right)^{\vee}$ vanishes, $0 \rightarrow\left(K_{1}^{*}\right)^{\vee} \rightarrow\left(V(\mathbf{1})^{*}\right)^{\vee} \rightarrow V \rightarrow 0$ gives

$$
H^{\ell-1}(V) \cong H^{\ell}\left(\left(K_{1}^{*}\right)^{\vee}\right) \cong H^{-\ell}\left(K_{1}^{*}\right)^{\vee} \quad, \quad \text { for all } \ell \text {. }
$$

$K_{1}^{*}$ is filtered with graded components $L_{2}, \ldots, L_{n}$ so that the cohomology of $K_{1}^{*}$ vanishes if the cohomology of the $L_{i}$ vanishes. Hence $H^{-\ell}\left(K_{1}^{*}\right)=0$ unless $-\ell \notin\{-2,-3, \ldots,-n\}$ and $H^{\nu}(V)=0$ for all $\nu \leq 0$ and all $\nu \geq n$. On the other hand $H^{\nu}\left(\operatorname{Ber} \otimes S^{n-1}\right)=0$ for $\nu \neq 1$ and $H^{1}\left(B e r \otimes S^{n-1}\right)=$ $\mathbf{1} \oplus\left(\operatorname{Ber} \otimes S^{n-1}\right)$. Since $H^{\nu}(V)=0$, if $H^{\nu}(\mathbf{1})=0$ and $H^{\nu}\left(B e r \otimes S^{n-1}\right)=0$, therefore $H^{\nu}(V)=0$ unless $\nu=1$.

Applying $(n-1)$ times the functor $D S$ to $D S(V) \in \mathcal{R}_{n-1}$, the last lemma gives

Lemma 28.5. If we apply $n$ times the functor $D S$ to $V=\left(Q_{1}^{*}\right)^{\vee}$ in $\mathcal{R}_{n}$, we obtain that

$$
D S \circ D S \circ \cdots \circ D S(V)=\bigoplus_{\nu=0}^{n-2} k[-1-2 \nu]
$$

in $\mathcal{R}_{0}$ is concentrated in the degrees $1,3, \cdots, 2 n-3$.

The Leray type spectral sequences therefore imply the following result

Corollary 28.6. For the module $V=\left(Q_{1}^{*}\right)^{\vee}$ in $\mathcal{R}_{n}$, defining a nontrivial extension between 1 and $\operatorname{Ber} \otimes S^{n-1}$, we have

$$
D S_{n, 0}^{\ell}(V)=0 \text { and } \omega_{n, 0}^{\ell}(V)=0 \quad \text { for } \ell \leq 0 \text {. }
$$

\section{STRICT MORPHISMS}

Recall the functor $\omega: T_{n} \rightarrow$ svec $_{k}$ defined by $\omega=\omega_{n, 0}$. A morphisms $q: V \rightarrow W$ in $T_{n}$ will be called a strict epimorphism, if the following holds

(1) $q$ is surjective.

(2) $\omega(q)$ is surjective. 
For a module $Z$ in $T_{n}$ and semisimple $L$ and

$$
q: Z \rightarrow L
$$

we make the following

Assumption (S). The induced morphism

$$
\omega(q): \omega(Z) \rightarrow \omega(L)
$$

is surjective, i.e. q is a strict epimorphism.

Of course (S) holds for irreducible $Z$. In the special case $L=\mathbf{1}$ condition (S) is equivalent to $\omega(q) \neq 0$. We denote the cosocle of $Z$ by $C$.

For any submodule $U \subseteq \operatorname{Kern}(q)$ the map $q: Z \rightarrow L$ factorizes over the quotient $p: Z \rightarrow V=Z / U$ and induces the analogous morphism $q_{V}: V \rightarrow$ $L \hookrightarrow \operatorname{cosocle}(Z / U)$. Hence

$$
q=q_{V} \circ p \quad, \quad \omega_{n, i}(q)=\omega_{n, i}\left(q_{V}\right) \circ \omega_{n, i}(p) .
$$

implies: $\omega_{n, i}(q)$ is surjective $\Longrightarrow \omega_{n, i}\left(q_{V}\right)$ is surjective. For $i=0$ thus

- If $Z$ is indecomposable, then $V$ is indecomposable.

- Condition $(S)$ for $q$ implies condition $(S)$ for $q_{V}$.

- $\omega\left(q_{V}\right)=0$ implies $\omega(q)=0$.

Indecomposable $Z$. Now assume $Z$ is indecomposable and has upper Loewy length $m \geq 2$. If $m \geq 3$, there exists a submodule $U \subset Z$ such that $V=Z / U$ has Loewy length 2 and such that $V$ again is indecomposable and satisfies assumption (S). So $V$ has Loewy length two and is indecomposable with cosocle $C$. Then $\left(V, q_{V}\right)$ is a nontrivial extension

$$
0 \rightarrow S \rightarrow V \rightarrow C \rightarrow 0
$$

with semisimple socle $S$ decomposing into irreducible summands $S_{\nu}$ and cosocle $C$. The map $q$ is obtained from a projection map $\operatorname{pr}_{L}: C \rightarrow L$ by composition with the canonical map $V \rightarrow C$. Since $V$ is indecomposable with cosocle $C$, all extensions $\left(V_{\nu}, q_{\nu}\right)$ obtained as pushouts

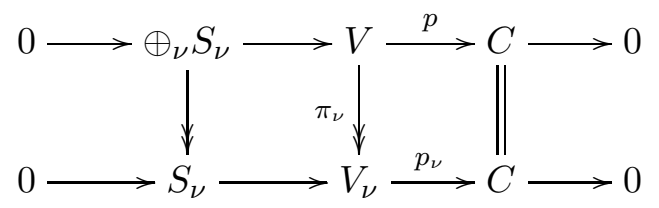


must be nontrivial extensions. All $V_{\nu}$ again satisfy condition (S): Indeed $\operatorname{Im}(\omega(q)) \subseteq \operatorname{Im}\left(\omega\left(q_{\nu}\right)\right)$

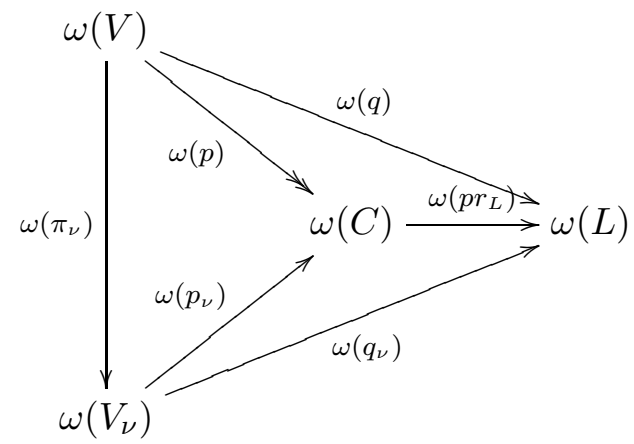

The projection $p r_{L}: C \rightarrow L$ splits by an inclusion $i_{L}: L \rightarrow C$, since $C$ is semisimple. Hence $C \cong L \oplus L^{\prime}$ so that $p r_{L}$ and $i_{L}$ are considered as the canonical projection resp. inclusion for the first summand.

Since $V$ is indecomposable, $\operatorname{Ext}^{1}\left(L, S_{\nu}\right) \neq 0$ holds for at least one $S_{\nu}$. Now divide by the submodule $U^{\prime} \subset S$ generated by all $S_{\nu}$ with the property $\operatorname{Ext}^{1}\left(L, S_{\nu}\right)=0$ and obtain $V^{\prime}=V / U^{\prime}$. Then divide by the maximal submodule $U^{\prime \prime}$ of $L^{\prime}$ that splits in $V^{\prime}$. Then $V^{\prime} / U^{\prime \prime}$ is indecomposable and the map $q$ factorizes over this quotient and satisfies condition $S$.

Resume. Suppose $Z$ is indecomposable but not irreducible, $q: Z \rightarrow L$ satisfies condition (S), the cosocle of $Z$ is $C=L \oplus L^{\prime}$. Then there exists a quotient $V$ of $Z$ and a quotient $\tilde{L}$ of $L^{\prime}$ such that

$$
0 \longrightarrow S \longrightarrow V \stackrel{p}{\longrightarrow} L \oplus \tilde{L} \longrightarrow 0
$$

with

- $V$ is indecomposable,

- $S$ is irreducible such that $\operatorname{Ext}^{1}(L, S) \neq 0$ and $\operatorname{Ext}^{1}(\tilde{L}, S) \neq 0$,

- the map $q=p r_{L} \circ p$ satisfies condition (S).

The irreducible representations $X \neq 1$ with the property $\operatorname{Ext}^{1}(X, S) \neq 0$ will be called descendants of $S$.

In the situation of the resume we get the extensions $E=E_{S}^{L}$ and $\tilde{E}=E_{S}^{\tilde{L}}$ defined by submodules of $V$. Hence $V / E_{S}^{L} \cong \tilde{L}$ and $V / E_{S}^{\tilde{L}} \cong L$ and we get the following exact sequences 


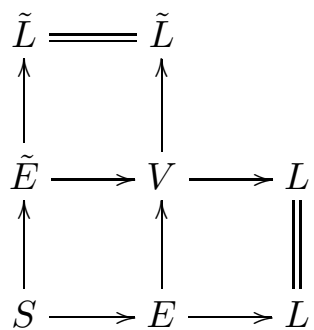

One of the potential candidates for $S_{\nu}$ is the irreducible representation $L(\lambda-\mu)$ that appears in the second upper Loewy level of the Kac module $V(\lambda)$. Indeed this follows from lemma 10.5, since $H_{D}(V(\lambda))=0$. Since $Z_{\nu}$ is indecomposable, $Z_{\nu}$ is in this case a highest weight representation of weight $\lambda$. This is clear, because all weights of $Z_{\nu}$ are in $\lambda-\sum_{\alpha \in \Delta_{n}} \mathbb{Z} \cdot \alpha$. By corollary 10.4 a highest representation $V$ contains a (nontrivial) highest weight subrepresentation $W$ of weight $\lambda-\mu$ only if $H_{D}(V)$ has trivial weight space $H_{D}(V)_{\bar{\lambda}}$. For $V=Z_{\nu}$ as above this gives a contradiction, if $S_{\nu}=$ $L(\lambda-\mu)$ occurs in the socle of $Z$. Indeed, notice that $H_{D}(L)$ contains $L(\bar{\lambda})$ by lemma 10.2. By condition (S) then also $H_{D}(Z)$ contains $L(\bar{\lambda})$. So by corollary $10.4 L(\lambda-\mu)$ is not contained in $Z_{\nu}$. This proves

Lemma 29.1. Suppose $Z$ is an (indecomposable) module with irreducible maximal atypical cosocle $L=L(\lambda)$. If $Z$ satisfies condition $(S)$, then the second layer of the upper Loewy filtration of $Z$ does not contain the irreducible module $L(\lambda-\mu)$.

A case of particular interest is $L=1$. Fix some irreducible $S$ with the property $\operatorname{Ext}_{\mathcal{R}_{n}}(S, \mathbf{1}) \neq 0$. In section 31 we will show for $L=\mathbf{1}$ that $\omega^{0}\left(q_{E}\right)=0$ (lemma 31.4).

\section{THE MODULE $R\left((n)^{n}\right)$}

We describe a certain maximal atypical mixed tensor for $n \geq 2$.

We recall some terminology from [BS11]. Given weights $\lambda, \mu \sim \alpha$ in the same block one can label the cup diagram $\lambda$ resp. the cap diagram $\mu$ with $\alpha$ to obtain $\underline{\lambda} \alpha$ resp. $\alpha \bar{\mu}$. These diagrams are by definition consistently oriented if and only if each cup resp cap has exactly one $\vee$ and one $\wedge$ and all the rays labelled $\wedge$ are to the left of all rays labelled $\vee$. Set $\lambda \subset \alpha$ iff $\lambda \sim \alpha$ and $\underline{\lambda} \alpha$ is consistently oriented.

A crossingless matching is a diagram obtained by drawing a cap diagram underneath a cup diagram and then joining rays according to some order-preserving bijection between the vertices. Given blocks $\Delta, \Gamma$ a $\Delta \Gamma$ matching is a crossingless matching $t$ such that the free vertices (not part of 
cups, caps or lines) at the bottom are exactly at the position as the vertices labelled $\circ$ or $\times$ in $\Delta$; and similarly for the top with $\Gamma$. Given a $\Delta \Gamma$-matching $t$ and $\alpha \in \Delta$ and $\beta \in \Gamma$, one can label the bottom line with $\alpha$ and the upper line with $\beta$ to obtain $\alpha t \beta$. $\alpha t \beta$ is consistently oriented if each cup resp cap has exactly one $\vee$ and one $\wedge$ and the endpoints of each line segment are labelled by the same symbol. Notation: $\alpha \rightarrow^{t} \beta$.

For $t$ a crossingless $\Delta \Gamma$ and $\lambda \in \Delta, \mu \in \Gamma$ label the bottom and the upper line as usual. The lower reduction $\operatorname{red}(\underline{\lambda} t)$ is the cup diagram obtained from $\underline{\lambda} t$ by removing the bottom number line and all connected components that do not extend up to the top number line.

Theorem 30.1. [BS12b], Thm 3.4. and [BS10a], Thm 4.11: In $K_{0}\left(\mathcal{R}_{n}\right)$ the mixed tensor $R(\lambda)$ attached to the bipartition $\lambda$ satisfies

$$
[R(\lambda)]=\sum_{\mu \subset \alpha \rightarrow^{t} \mathbf{1}, \operatorname{red}(\underline{\mu} t)=\underline{\mathbf{1}}}[L(\mu)]
$$

where $t$ is a fixed matching determined by $\lambda$ between the block $\Gamma$ of 1 and the block $\Delta$ of $\lambda^{\dagger}$ [BS12b], 8.18. If $L(\mu)$ is a composition factor of $R(\lambda)$, its graded composition multiplicities are given by

$$
\sum_{\mu}\left(q+q^{-1}\right)^{n_{\mu}}[L(\mu)]
$$

where $n_{\mu}$ is the number of lower circles in $\underline{\mu t}$.

Lemma 30.2. The module $R=R\left((n)^{n}\right)$ in $\mathcal{R}_{n+r+1}, r \geq 0$, has Loewy length $2 n+1$ with socle and cosocle equal to 1 . We have $\left.D S\left(R\left(n^{n}\right)\right)\right)=$ $R\left(n^{n}\right)$. If $r=0, D S(R)=P(\mathbf{1}) . R$ contains 1 with multiplicity $2^{2 n}$. It contains the irreducible module $L(h)=[n, 1, \ldots, 1,0, \ldots, 0]$ (with 1 occurring $n$-1-times) in the second Loewy layer. The multiplicity of $L(h)$ in $R$ is $2^{2(n-1)}$. It contains the module $[n, n, \ldots, n, 0, \ldots, 0]$ as the constituent of highest weight in the middle Loewy layer with multiplicity 1. It does not contain the modules $B S^{n-1}=[n, 1, \ldots, 1], B S^{n}=[n+1,1, \ldots, 1]$, $[n, 1, \ldots, 1,-1]$ and $[n, 1, \ldots, 1,-1, \ldots,-1]$ (with 1 occurring $n-1$-times) as composition factors.

Proof. The Loewy length of a mixed tensor is $2 d(\lambda)+1$ (where $d(\lambda)$ is the number of caps) and $d\left((n-1)^{n-1}\right)=n-1$ [Hei14]. The composition factors of $R$ are given as a sum $\sum_{\mu}\left(q+q^{-1}\right)^{n_{\mu}}[L(\mu)]$. For our choice of $\lambda=(n-1)^{n-1}$ the matching is given by [Hei14] (picture for $n=4$ ) 


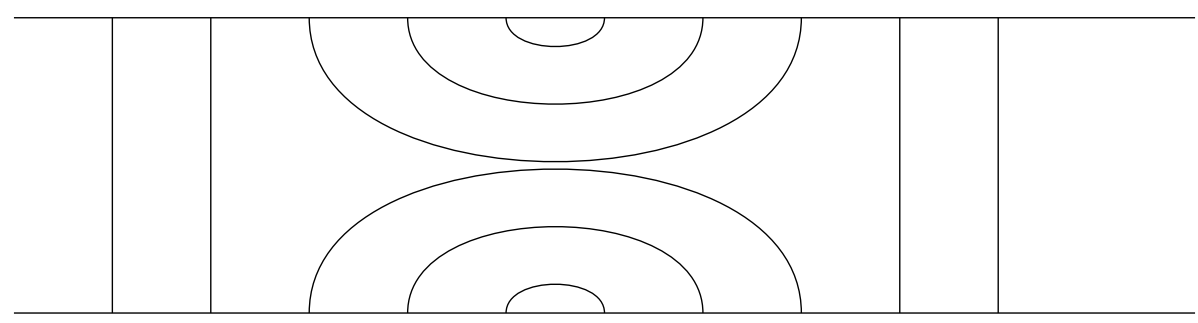

with $n$ caps and where the rightmost vertex in a cap is at position $n$. The irreducible module in the socle and cosocle is easily computed from the rules of the section 14 . The weight

$$
h=(n, 1, \ldots, 1,0 \mid 0,-1, \ldots,-1, n-n)
$$

easily seen to satisfy $h \rightarrow^{t} \mathbf{1}, \operatorname{red}(\underline{h} t)=\underline{\mathbf{1}}$, hence occurs as a composition factor. The number of lower circles in the lower reduction $\underline{h} t$ is $n-1$, hence $L(h)$ occurs with multiplicity $2^{2(n-1)}$. If we number the Loewy layers starting with the socle by $1, \ldots, 2 n+1, L(h)$ occurs in the $2 k$-th Loewy layer $(k=1, \ldots, n)$ with multiplicity $\left(\begin{array}{l}n-1 \\ k-1\end{array}\right)$. Likewise for 1 with $n_{\mathbf{1}}=$ $n-1$. We note: A weight $\mu$ can only satisfy $\operatorname{red}(\underline{\lambda} t)=\underline{1}$ if the vertices $-n,-n-1, \ldots,-n-r$ (the first vertices left of the caps) are labelled by $\vee$. Hence:

- $B S^{n-1}$ does not occur as a composition factor. The vertex $-n$ is labelled by $\wedge$.

- $[n, 1, \ldots, 1,-1]$ does not occur as a composition factor. The vertex $-n$ is labelled by $\wedge$

- $[n+1,1, \ldots, 1]$ does not occur as a composition factor since all composition factors $\left[\mu_{1}, \ldots, \mu_{n}\right]$ satisfy $\mu_{1} \leq n$ since $[n, \ldots, n, 0, \ldots, 0]$ is the constituent of highest weight.

Remark. In particular the constituent 1 occurs with the same multiplicity as in $P(\mathbf{1}) \in \mathcal{R}_{n}$.

Remark. The module $R\left(n^{n}\right)$ can be obtained as follows. Let $\left\{n^{n}\right\}$ be the covariant module to the partition $\left(n^{n}\right)$ and $\left\{n^{n}\right\}^{\vee}$ its dual. Then $R\left(n^{n}\right)$ is the projection on the maximal atypical block of $\left\{n^{n}\right\} \otimes\left\{n^{n}\right\}^{\vee}$.

Example. For $G l(3 \mid 3)$ the Loewy structure of the module $R\left(2^{2}\right)$ is

$$
\left(\begin{array}{c}
{[0,0,0]} \\
{[1,0,0] \oplus[2,1,0]} \\
{[2,0,0] \oplus[2,-1,-1] \oplus[0,0,0] \oplus[0,0,0] \oplus[1,1,0] \oplus[2,2,0]} \\
{[1,0,0] \oplus[2,1,0]} \\
{[0,0,0]}
\end{array}\right) .
$$




\section{THE BASIC HOOK REPRESENTATIONS $S$}

The case $L=1$. Suppose $Z$ has cosocle 1 and the projection $q: Z \rightarrow \mathbf{1}$ satisfies condition (S). If $Z$ is not simple, we constructed objects $V_{\nu}$ with cosocle 1 and simple socle $S_{\nu}=\operatorname{Ker}\left(q_{\nu}\right)$. In this situation $\operatorname{Ext}_{\mathcal{R}_{n}}^{1}\left(\mathbf{1}, S_{\nu}\right) \neq$ 0 .

Proposition 31.1. For any nontrivial extension

$$
0 \longrightarrow S_{\nu} \longrightarrow V \longrightarrow 1 \longrightarrow 0
$$

the vectorspace $\omega_{n, 0}^{0}(V)$ is zero (for simple $\left.S_{\nu}\right)$. Hence $\omega(q): \omega(V) \rightarrow \omega(\mathbf{1})$ is the zero map.

For the proof we use several lemmas. Finally lemma 31.4 proves the proposition.

Lemma 31.2. Up to isomorphism there are $n+1$ irreducible modules $L$ in $\mathcal{R}_{n}$ such that $\operatorname{Ext}^{1}(\mathbf{1}, L) \neq 0$. They are

(1) $L_{n}(n)=B e r_{n} \otimes S^{n-1}$ and

(2) its dual $L_{n}(n)^{\vee} \cong[0, . ., 0,-1]$, and for

(3) $i=1, . ., n-1$ the basic selfdual representations

$$
L_{n}(i)=[i, 1, \cdots, 1,0, \cdots, 0]
$$

(with $n-i$ entries 0$)$.

In all cases $\operatorname{dim}\left(\operatorname{Ext}^{1}(L, 1)\right)=1$. Furthermore

$$
D S_{n, j}\left(L_{n}(i)\right)=L_{j}(i)
$$

holds for $i<j \leq n$ and

$$
D S_{n, i}\left(L_{n}(i)\right)=L_{i}(i) \oplus L_{i}(i)^{\vee} \oplus Y
$$

where $Y \neq 1$ is an irreducible module with $\operatorname{Ext}^{1}(\mathbf{1}, Y)=0$ and sector structure

$$
\left[\vee_{-n}, \wedge_{-n+1}\right] \wedge_{-n+2}[-n+3, \ldots, n-2] \wedge_{n-1}\left[\vee_{n}, \wedge_{n+1}\right]
$$

Example. $L_{n}(1)=S^{1}$.

Proof. $L^{*} \cong L$ for irreducible objects $L$ implies $\operatorname{Ext}^{1}(\mathbf{1}, L) \cong \operatorname{Ext}^{1}(L, \mathbf{1})$. Furthermore $\operatorname{Ext}^{1}(L, \mathbf{1}) \cong \operatorname{Ext}^{1}\left(\left(L^{*}\right)^{\vee}, \mathbf{1}\right)$ and $L=L^{*}$, hence

$$
\operatorname{Ext}^{1}(L, \mathbf{1}) \cong \operatorname{Ext}^{1}\left(L^{\vee}, \mathbf{1}\right) \text {. }
$$

By [BS10a], cor. 5.15 for $L=L(\lambda)$

$$
\operatorname{dim} \operatorname{Ext}^{1}(L(\lambda), \mathbf{1})=\operatorname{dim} \operatorname{Ext}^{1}(V(\lambda), \mathbf{1})+\operatorname{dim} \operatorname{Ext}^{1}(V(0), L(\lambda))
$$

holds. Since 1 is a Kostant weight, there exists a unique weight $\lambda$ characterized by $\lambda \leq 0$ (Bruhat ordering) and $l(\lambda, 0)=1$ in the notations of 
loc. cit. lemma 7.2, such that $\operatorname{dim} \operatorname{Ext}^{1}(V(\lambda), \mathbf{1}) \neq 0$. One easily shows $L(\lambda) \cong[0, . ., 0,-1]$.

On the other hand $\operatorname{dim} \operatorname{Ext}(V(0), L(\lambda)) \neq 0$ implies $0<\lambda$ (see the explanations preceding loc. cit. (5.3) and loc. cit. lemma 5.2.(i)). Then for any pair of adjacent labels $i, i+1$ of $\rho$ of type $i=\vee, i+1=\wedge$ we write $\rho \in \Lambda^{\vee}, \wedge$, if the labels of $\rho$ at $i, i+1$ are the same $i=\vee, i+1=\wedge$. Then lemma 5.2(ii) of loc. cit. gives

$$
\operatorname{dim}\left(\operatorname{Ext}_{\mathcal{R}_{n}}^{1}(V(\rho), L(\lambda))= \begin{cases}\operatorname{dim}\left(\operatorname{Ext}_{\mathcal{R}_{n-1}}^{1}\left(V\left(\rho^{\prime}\right), L\left(\lambda^{\prime}\right)\right)\right. & \text { if } \lambda \in \Lambda^{\vee, \wedge} \\ \operatorname{dim}\left(\operatorname{Hom}_{\mathcal{R}_{n}}\left(V\left(\rho^{\prime \prime}\right), L(\lambda)\right)\right. & \text { otherwise }\end{cases}\right.
$$

Here $\lambda^{\prime}, \mu^{\prime}$ are obtained from $\lambda, \mu$ by deleting $i, i+1$, and $\rho^{\prime \prime}$ is obtained by transposing the labels at $i, i+1$.

This shows our assertion, since for

$$
L(\rho)=\mathbf{1}
$$

there is a unique pair of such neighbouring indices for

$$
\left[\vee_{-n+1}, \ldots, \vee_{0}, \wedge_{1}, \ldots, \wedge_{n}\right]
$$

namely at the position $(i, i+1)=(0,1)$. We now assume $n \geq 2$. Then switching this pair gives $L_{n}(1)$ below. Freezing then also $(-1, ., ., 2)$ gives $L_{n}(2)$ and so on. Hence applying this lemma of loc. cit. several times will prove our first claim. Indeed, as long as we freeze less than $n-2$ pairs, we end up for every $j$ from $1, \ldots, n-1$ with a representation $L_{n}(j)$. It has only one sector

$\left[\vee_{1-n}, \ldots, \vee_{-j-1}\left[\vee_{-j} \wedge_{-j+1}\right]\left[\vee_{-j+2}, \ldots, \vee_{0}, \wedge_{1}, \ldots, \wedge_{j-1}\right]\left[\vee_{j}, \wedge_{j+1}\right] \wedge_{j+2}, \ldots, \wedge_{n}\right]$

In addition, if we freeze $n-1$ pairs we end up with $L_{n}(n)$ with the sector structure

$$
\left[\vee_{2-n}, \vee_{3-n}, \ldots ., \wedge_{n-2}, \wedge_{n-1}\right]\left[\vee_{n}, \wedge_{n+1}\right] .
$$

Indeed $L_{n}(n) \cong B e r_{n} \otimes S^{n-1}$.

The remaining assertions now follow from theorem 16.1, since $L_{n+1}(n)$ has sectors

$$
S_{1} S_{2} S_{3}=\left[\vee_{-n}, \wedge_{-n+1}\right][-n+2, \ldots, n-1]\left[\vee_{n}, \wedge_{n+1}\right] .
$$

Hence $D S\left(L_{n+1}(n)\right)=\left(\operatorname{Ber} \otimes S^{n-1}\right) \oplus\left(\operatorname{Ber} \otimes S^{n-1}\right)^{\vee} \oplus Y$ for $Y$ with sector structure $\left[\vee_{-n}, \wedge_{-n+1}\right][-n+3, \ldots, n-2] \wedge_{n-1}\left[\vee_{n}, \wedge_{n+1}\right]$.

Basic cases. For a nontrivial extension

$$
0 \longrightarrow L_{n}(i) \longrightarrow V \longrightarrow \mathbf{1} \longrightarrow 0
$$


first suppose $S=L_{n}(i)$ is basic, so $i \in\{1, \ldots, n-1\}$. Since $\left(L_{n}(i)^{*}\right)^{\vee} \cong$ $L_{n}(i)^{\vee} \cong L_{n}(i)$ for $i<n,\left(V^{*}\right)^{\vee}$ again defines a nontrivial extension

$$
0 \longrightarrow L_{n}(i) \longrightarrow\left(V^{*}\right)^{\vee} \longrightarrow \mathbf{1} \longrightarrow 0
$$

We now use $D S\left(L_{n}(i)\right)=L_{n-1}(i)$ for $1 \leq i<n-1$. In $T_{n-1}$ the induced long exact sequence

$$
H^{-1}(\mathbf{1}) \longrightarrow H^{0}(S) \longrightarrow H^{0}(V) \longrightarrow H^{0}(\mathbf{1}) \longrightarrow H^{1}(S)
$$

remains exact, since $H^{\ell}(S)=L_{n-1}(i)$ for $\ell=0$ and is zero otherwise and similarly $H^{\ell}(\mathbf{1})=\mathbf{1}$ for $\ell=0$ and is zero otherwise. In other words for basic $S$ we obtain from the given extension in $\mathcal{R}_{n}$ an exact sequence in $\mathcal{R}_{n-1}$

$$
0 \longrightarrow L_{n-1}(i) \longrightarrow D S(V) \longrightarrow 1 \longrightarrow 0 \text {. }
$$

Repeating this $n-i$ times we obtain an exact sequence

$$
0 \longrightarrow L_{i}(i) \oplus L_{i}(i)^{\vee} \oplus Y \longrightarrow D S_{n, i}(V) \longrightarrow \mathbf{1} \longrightarrow 0
$$

Since $\operatorname{Ext}^{1}(\mathbf{1}, Y)=0$ this implies

$$
D S_{n, i}(V)=E \oplus Y
$$

for some selfdual module $E$ defining an extension between 1 and $L_{i}(i) \oplus$ $L_{i}(i)^{\vee}$. We claim that this exact sequence does not split in $\mathcal{T}_{i}$.

Proposition 31.3. Suppose $r$ is an integer $\geq 0$. For an indecomposable module $V$ defining a nontrivial extension between 1 and $L_{n+1+r}(n)$ in $\mathcal{R}_{n+r+1}$, the object $(D S)^{\circ r+1}(V)$ decomposes into the direct sum of the irreducible module $Y$ from above and an indecomposable extension module $E$ in $\mathcal{R}_{n}$.

Proof. Note that any two such indecomposable extensions define isomorphic modules $V$, since the relevant Ext-groups are one-dimensional. We assume $r=0$ for simplicity. Since the constituents $L_{n+1}(n)$ and 1 of $V$ are basic, this implies $D S(V)=H^{0}(V)=Y \oplus E$. If the module $E$ is not indecomposable, it is semisimple (for this use Tannaka duality). We proceed as follows:

For the mixed tensor $R=R_{n^{n}}$ in $\mathcal{R}_{n+1}$ we know that its image $D S\left(R_{n^{n}}\right)$ is the projective hull $P(\mathbf{1})$ of $\mathbf{1}$ in $\mathcal{R}_{n}$ and $P(\mathbf{1})$ is an indecomposable module with top 1. The module $R_{n^{n}}$ admits as quotient an indecomposable module $V$ defining a nontrivial extension between 1 (the top of $R$ ) and the module $L_{n+1}(n)$ (which sits in the second layer of the Loewy filtration of $R$ ). Hence $R / K \cong V$ for some submodule $K$ of $R$. We claim that

$$
0 \longrightarrow H^{0}(K) \stackrel{i}{\longrightarrow} H^{0}(R) \stackrel{p}{\longrightarrow} E \oplus Y \longrightarrow H^{1}(K) \longrightarrow 0
$$


is exact and $H^{\nu}(K)=0$ for $\nu \neq 0,1$. For this use $H^{\bullet}(V)=H^{0}(V)$ and $H^{\nu}(R)=P(\mathbf{1})$ for a unique $\nu$. If $\nu \neq 0$, then $H^{\nu}(K) \rightarrow H^{\nu}(R)=P(\mathbf{1})$ would be surjective and therefore $H^{\nu}(K)=P(\mathbf{1}) \oplus$ ?. We exclude this later. So suppose for the moment $\nu=0$.

The image of $p$ can not contain the irreducible module $Y \neq 1$, since the top of $P(\mathbf{1})$ is $\mathbf{1}$. If $E$ splits, it is semisimple. Then the image of $p$ can not contain $E$ either, since again this would contradict that $P(\mathbf{1})$ has top 1. Therefore the image of $p$ is $\mathbf{1}$ or zero, if $E$ splits. This leads to a contradiction:

Look at all constituents $X$ of $R$ with $B e r \otimes S^{n-1}$ in $H^{\nu}(X)$ for $\nu=-1,0,1$. These $X$ are isomorphic to the following irreducible modules $X_{-1}, X_{0}, X_{1}$ with $\operatorname{Ber} \otimes S^{n-1}$ occuring in $H^{i}\left(X_{i}\right)$ respectively: the basic module $X_{0}=$ $L_{n+1}(n)$ with sector structure $[-n,-n+1][-n+2, \ldots, n-1][n, n+1]$ and $X_{1}$ with sector structure $[1-n[2-n, \ldots, n-1][n, n+1] n+2]$ and $X_{-1}$ with sector structure $[-n-1,-n] \boxminus[2-n, \ldots, n-1][n, n+1]$. Then $\operatorname{Ber} \otimes S^{n-1}$ occurs in $H^{i}\left(X_{i}\right)$ for $i=0, \pm 1$.

Let $F^{i}($.$) denote the descending Loewy filtration. For a module Z$ let $m(Z)$ denote the number of Jordan-Hölder constituents of $Z$ that are isomorphic to $B e r^{\otimes} S^{n-1}$. Next we use that for all $i$

$$
X_{1}, X_{-1} \text { does not occur in the } g r_{F}^{i}(R) \text {. }
$$

Indeed according to section 30 all irreducible constituents $[\lambda]$ satisfy the property $\lambda_{n+1}=0$ except for one given by $[n,-n+1, \ldots,-n+1]$. Therefore $m\left(H^{ \pm 1}\left(g r_{F}^{i}(R)\right)\right)=0$ and hence $m\left(H^{1}\left(F^{i}(X)\right)\right)=0$. Since also $m\left(H^{-1}\left(g r_{F}^{i}(R)\right)\right)=0$, then

$$
H^{-1}\left(g r_{F}^{i}(R)\right) \rightarrow H^{0}\left(F^{i}(R)\right) \rightarrow H^{0}\left(F^{i-1}(R)\right) \rightarrow H^{0}\left(g r_{F}^{i}(R)\right) \rightarrow H^{1}\left(F^{i}(R)\right)
$$

implies $m\left(H^{0}\left(F^{i}(R)\right)\right)=m\left(H^{0}\left(F^{i-1}(R)\right)\right)+m\left(H^{0}\left(g r_{F}^{i}(R)\right)\right)$. For small $i$ we have $F^{i}(R)=R$ and therefore

$$
m\left(H^{0}(R)\right)=\sum_{i} m\left(H^{0}\left(g r_{F}^{i}(R)\right)\right) .
$$

They same argument then applies for the submodule $K$ of $R$. Hence

$$
m\left(H^{0}(K)\right)=m\left(H^{0}(R)\right)-1
$$

by counting the multiplicities of $X_{0}$ in $K$ resp. $R$. Hence the image of $p$ must contain $\operatorname{Ber} \otimes S^{n-1}$ and hence $E$ is an indecomposable quotient of $P(\mathbf{1})$.

Now let us adress the assertion $\nu=0$ from above. If $\nu \neq 0$, then $H^{0}(K) \cong$ $P(\mathbf{1}) \oplus$ ? gives a contradiction using the same counting argument. 
In the case $r>0$ one uses the same kind of argument. Again the extension defined by $V$ in $\mathcal{R}_{n+r+1}$ can be realized as a quotient of $R=R_{n^{n}}$ in $\mathcal{R}_{n+r+1}$. The argument is modificatis modificandis the same.

The non-basic cases. For a nontrivial extension in $T_{n}$ of the form

$$
0 \longrightarrow L_{n}(n) \longrightarrow V \longrightarrow \mathbf{1} \longrightarrow 0 \text {. }
$$

we get a dual nontrivial extension

$$
0 \longrightarrow[0, \ldots, 0,-1] \longrightarrow\left(V^{*}\right)^{\vee} \longrightarrow 1 \longrightarrow 0
$$

In lemma 28.1 and lemma 28.2 we defined $Q_{a}$, which for $a=1$ defines a nontrivial extension between 1 and $L_{n}(n)^{\vee}$. Since $\operatorname{dim}\left(\operatorname{Ext} t^{1}\left(\mathbf{1}, L_{n}(n)^{\vee}\right)\right)=$ 1 , we get

$$
\left(V^{*}\right)^{\vee} \cong Q_{1}
$$

By corollary 28.6 we get $D S_{n, 0}^{\ell}(V)=0$ and $\omega_{n, 0}^{\ell}(V)=0$ for all $\ell \leq 0$. Similarly by duality $D S_{n, 0}^{\ell}\left(\left(V^{*}\right)^{\vee}\right)=0$ for $\ell \geq 0$. This implies $\omega_{n, 0}^{\ell}\left(\left(V^{*}\right)^{\vee}\right)=$ 0 for $\ell \geq 0$.

Finally consider the nontrivial extension $V_{i}$ between 1 and $L_{n}(i)$ in $\mathcal{R}_{n}$ and the nontrivial extension $D S_{n, i}\left(V_{i}\right)$ in $\mathcal{R}_{i}$ from above. It has the form $D S_{n, i}\left(V_{i}\right)=E \oplus Y$ for

$$
0 \rightarrow L_{i}(i) \oplus L_{i}(i)^{\vee} \rightarrow E \rightarrow \mathbf{1} \rightarrow 0 .
$$

The module $E=D S_{n, i}\left(\left(V_{i}\right) / Y\right.$ is the pullback of a nontrivial extension of 1 by $L_{i}(i)$

$$
0 \rightarrow L_{i}(i) \rightarrow E_{1} \rightarrow \mathbf{1} \rightarrow 0
$$

and of a nontrivial extension of 1 by $\left(L_{i}(i)\right)^{\vee}$

$$
0 \rightarrow\left(L_{i}(i)\right)^{\vee} \rightarrow E_{2} \rightarrow \mathbf{1} \rightarrow 0 .
$$

Hence there exists an exact sequence

$$
0 \rightarrow D S_{n, i}\left(V_{i}\right) / Y \rightarrow E_{1} \oplus E_{2} \rightarrow \mathbf{1} \rightarrow 0
$$

so that

$$
\rightarrow D S_{i, 0}^{-1}(\mathbf{1}) \rightarrow D S_{i, 0}^{0}\left(D S_{n, i}\left(V_{i}\right) / Y\right) \rightarrow D S_{i, 0}^{0}\left(E_{1}\right) \oplus D S_{i, 0}^{0}\left(E_{2}\right) \rightarrow
$$

is exact. Since $D S_{i, 0}^{0}\left(E_{1}\right)=0$ and $D S_{i, 0}^{0}\left(E_{2}\right)=0$ by corollary 28.6 and since $D S_{i, 0}^{-1}(\mathbf{1})=0$, therefore $D S_{i, 0}^{0}\left(D S_{n, i}\left(V_{i}\right) / Y\right)=0$. Hence $\omega_{i, 0}^{0}\left(D S_{n, i}\left(V_{i}\right)\right)=$ $\omega_{i, 0}^{0}(Y)$. The Leray type spectral sequence

$$
D S_{i, 0}^{p}\left(D S_{n, i}^{q}\left(V_{i}\right)\right) \Longrightarrow D S_{n, 0}^{p+q}\left(V_{i}\right)
$$

degenerates, since $D S_{n, i}^{q}\left(V_{i}\right)=0$ for $q \neq 0$. Therefore also $\omega_{n, i}^{\bullet}\left(V_{i}\right) \cong$ $D S_{n, i}^{\bullet}\left(V_{i}\right)$. One can now argue as in the proof of lemma 8.4 to show

$$
\omega_{n, 0}^{0}\left(V_{i}\right)=\omega_{i, 0}^{0}\left(D S_{n, i}\left(V_{i}\right)\right)=\omega_{i, 0}(Y) .
$$


Since the map

$$
\omega_{i, 0}(q): E \oplus Y \longrightarrow \mathbf{1}
$$

is trivial on the simple summand $Y \neq 1$, the next lemma follows.

Lemma 31.4. For every nontrival extension

$$
0 \longrightarrow S \longrightarrow V \stackrel{q_{V}}{\longrightarrow} \mathbf{1} \longrightarrow 0
$$

of 1 by a simple object $S$ in $\mathcal{R}_{n}$ the map $\omega^{0}\left(q_{V}\right)$ vanishes.

The last lemma completes the proof of proposition 31.1. This implies the following main result.

Corollary 31.5. Suppose $Z$ is indecomposable and cosocle $(Z) \cong 1$. If the quotient map $q: Z \rightarrow \mathbf{1}$ is strict, then $q: Z \cong \mathbf{1}$.

Remark. A symmetric abelian tensor category in the sense of Deligne is semisimple if and only if $q: Z \rightarrow \mathbf{1}$, with cosocle of $Z$ isomorphic to $\mathbf{1}$, is an isomorphism.

Corollary 31.6. For a nontrivial extension $V$ between 1 and $L_{n}(n)$ or its dual $L_{n}(n)^{\vee}$

$$
H_{D}^{\nu}(V)=0 \quad, \quad \nu \neq 1
$$

holds, and hence the induced map $H_{D}(q): H_{D}(V) \rightarrow H_{D}(\mathbf{1})$ is trivial.

\section{REFERENCES}

[BKN09a] Boe, B. D. and Kujawa, J. R. and Nakano, D. K., Complexity and module varieties for classical Lie superalgebras, Int. Math. Res. Not., 2011 3, (2011)

[BKN09b] Boe, B. D. and Kujawa, J. R. and Nakano, D. K., Cohomology and support varieties for Lie superalgebras II, Proc. Lond. Math. Soc. (3), 98, 1, (2009)

[BKN10] Boe, B. D. and Kujawa, J. R. and Nakano, D. K., Cohomology and support varieties for Lie superalgebras, Trans. Am. Math. Soc., 362, 12, (2010)

[Bru03] Brundan, J., Kazhdan-Lusztig polynomials and character formulae for the Lie superalgebra $\mathfrak{g l}(m \mid n)$, J. Am. Math. Soc., 16, 1, (2003)

[BS10a] Brundan, J. and Stroppel, C., Highest weight categories arising from Khovanov's diagram algebra. II: Koszulity, Transform. Groups, 15, 1, (2010)

[BS11] Brundan, J. and Stroppel, C., Highest weight categories arising from Khovanov's diagram algebra. I: cellularity, Mosc. Math. J., 11, 4, (2011)

[BS12a] Brundan, J. and Stroppel, C., Highest weight categories arising from Khovanov's diagram algebra. IV: the general linear supergroup, J. Eur. Math. Soc. (JEMS), 14, 2, (2012)

[BS12b] Brundan, J. and Stroppel, C., Gradings on walled Brauer algebras and Khovanov's arc algebra, Adv. Math., 231, 2, (2012)

[BSch17] Bump, D. and Schilling A., Crystal bases. Representations and combinatorics., Hackensack, NJ: World Scientific, 2017

[CW11] Comes, J. and Wilson, B., Deligne's category Rep $\left(G L_{\delta}\right)$ and representations of general linear supergroups, Represent. Theory, 16, (2012) 
[Del07] Deligne, P., La catégorie des représentations du groupe symétrique $S_{t}$, lorsque $t$ n'est pas un entier naturel, Mehta, V. B. (ed.), Algebraic groups and homogeneous spaces. Proceedings of the international colloquium, Mumbai, India, January 6-14, 2004. 209-273, (2007)

[Dro09] Drouot, Fr., Quelques proprietes des representations de la super-algebre der Lie $\mathfrak{g l}(m, n), \mathrm{PhD}$ thesis, (2009)

[DS05] Duflo M. and Serganova V., On associated variety for Lie superalgebras, arXiv:math/0507198v1,(2005)

[Ger98] Germoni, J., Indecomposable representations of special linear Lie superalgebras, J. Algebra , 209, 2, (1998)

[GKPM11] Geer, N. and Kujawa, J. and Patureau-Mirand, B., Generalized trace and modified dimension functions on ribbon categories, Sel. Math., New Ser., 17, 2, (2011)

[GQS07] Goetz, G. and Quella, Th. and Schomerus, V., Representation theory of $\mathfrak{s l}(2 \mid 1)$, J. Algebra, 312, 2, (2007)

[Hei14] Heidersdorf, Th., Mixed tensors of the General Linear Supergroup, J.Algebra, 491, (2017)

[Hei15] Heidersdorf, Th., On representations of supergroups and their semisimple quotient, preprint, (2015)

[HW15a] Heidersdorf, Th. and Weissauer, R., Pieri type rules and $G l(2 \mid 2)$ tensor products, ArXiv e-prints, 1509.07443, (2015)

[HW15b] Heidersdorf, Th. and Weissauer, R., On classical tensor categories attached to the irreducible representations of the General Linear Supergroups $G l(n \mid n)$, preprint, (2015)

[HS53] Hochschild, G. and Serre, Jean-Pierre, Cohomology of Lie algebras., Ann. Math. (2), 57, 1953

[Kac78] Kac, V., Representations of classical Lie superalgebras, Differ. geom. Meth. math. Phys. II, Proc., Bonn 1977, Lect. Notes Math. 676, 597-626 (1978)

[Kuj11] Kujawa, J., The generalized Kac-Wakimoto conjecture and support varieties for the Lie superalgebra $\operatorname{osp}(m \mid 2 n)$, Recent developments in Lie algebras, groups and representation theory. 2009-2011 Southeastern Lie theory workshop series (2012)

[Mei13] Meinrenken E., Clifford algebras and Lie theory, Berlin: Springer, 2013

[Sa01] Sagan B., The symmetric group. Representations, combinatorial algorithms, and symmetric functions. 2nd ed., 2nd ed., New York, NY: Springer, 2001

[Sch79] Scheunert, M., The theory of Lie superalgebras. An introduction, 1979, Lecture Notes in Mathematics. 716. Berlin-Heidelberg-New York: Springer-Verlag. X, 271 p. (1979)

[Ser06] Serganova, V. Blocks in the category of finite-dimensional representations of $g l(m, n), 2006$

[GS10] Gruson, C. and Serganova, V., Cohomology of generalized supergrassmannians and character formulae for basic classical Lie superalgebras. Proc. Lond. Math. Soc. (3) 101 (2010)

[Ser10] Serganova, V., On the superdimension of an irreducible representation of a basic classical Lie super algebra, Supersymmetry in mathematics and physics. UCLA Los Angeles, USA 2010. Papers based on the presentations at the workshop, Februar 2010., Berlin: Springer (2011)

[Wei10] Weissauer, R., Monoidal model structures, categorial quotients and representations of super commutative Hopf algebras II: The case $G l(m, n)$, arXiv e-prints, 1010.3217 (2010) 